\title{
1
}

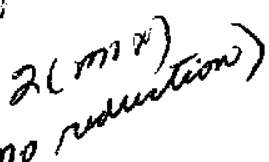

$n^{n^{2}}$

Scientific Uses

of the

Space Shuttle

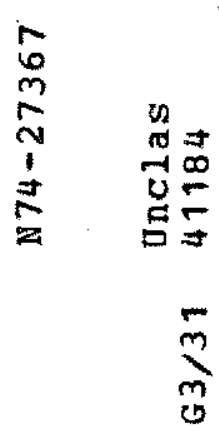

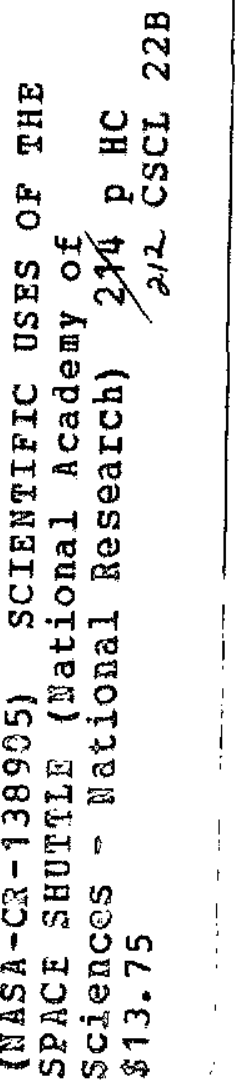




\section{Scientific Uses of the \\ Space Shuttle}

Space Science Board

National Research Council

NATIONAL ACADEMY OF SCIENCES

Washington, D.C. 
NOTICE: The project which is the subject of this report was approved by the Governing Board of the National Research Council, acting in behalf of the National Academy of Sciences. Such approval reflects the Board's judgment that the project is of national importance and appropriate with respect to both the purposes and resources of the National Research Council.

The members of the committee selected to undertake this project and prepare this report were chosen for recognized scholarly competence and with due consideration for the balance of disciplines appropriate to the project. Responsibility for the detailed aspects of this report rests with that committee.

Each report issuing from a study committee of the National Research Council is reviewed by an independent group of qualified individuals according to procedures established and monitored by the Report Review Committee of the National Academy of Sciences. Distribution of the report is approved, by the President of the Acadcmy, upon satisfactory completion of the review process. 


\section{Space Science Board}

Richard M. Goody, Chairman

James R. Arnold

E. Margaret Burbidge

George R. Carruthers

Robert E. Danielson

Herbert Friedman

Robert A. Helliwell

Norman H. Horowitz

Francis S. Johnson

Edward H. Kass

Robert B. Leighton

Joshua Menkes

Philip Morrison

Brian O'Brien

Robert A. Phinney

P. Buford Price

Frederick Seitz

John T. Shepherd

Roman Smoluchowski

Willis M. Hawkins, ex officio

David H. Garber, Executive Secretary 


\section{Participants}

John W. Findlay, National Radio Astronomy Observatory, Chairman

\section{A T L A R G E}

Richard M. Goody, Harvard University

Willis Hawkins, Lockheed Aircraft

Joshua Menkes, University of Colorado

George E. Solomon, TRW Systems

Charles H. Townes, University of California, Berkeley

H. C. van de Hulst, Leiden University, The Netherlands

\section{ATMOSPHERIC AND SPACE PHYSICS}

Thomas Donahue, University of Pittsburgh, Group Leader

B. Bertotti, University of Pavia, Italy

J. E. Blamont, Service d'Aeronomie, France

Sidney Bowhill, University of Illinois

Neil M. Brice, Cornell University

Donald Gurnett, University of Iowa

Robert Hudson, Lyndon B. Johnson Space Center

Francis S. Johnson, University of Texas at Dallas

Andrew Nagy, University of Michigan

Juan G. Roederer, University of Denver

Frederick L. Scarf, TRW Systems Group

\section{H I G H - E N E R G Y A S T R OPH Y S I C S}

Laurence E. Peterson, University of California, San Diego, Group Leader Hale Bradt, Massachusetts Institute of Technology

Carl Fichtel, Goddard Space Flight Center

Herbert Friedman, U.S. Naval Research Laboratory

Riccardo Giacconi, Center for Astrophysics

Kenneth Greisen, Cornell University

Frank B. McDonald, Goddard Space Flight Center 
Minoru Oda, Uníversity of Tokyo

K. Pinkau, Max-Planck-Institut für Extraterrestrische Physik, Germany

Rochus E. Vogt, California Institute of 'Technology

\section{N F R A R E D A S T R O NOM Y}

William F. Hoffmann, University of Arizona, Group Leader

Rudolf Hanel, Goddard Space Flight Center

Richard E. Jennings, University College, London

Gerry Neugebauer, California Institute of Technology

Stephen D. Price, Air Force Cambridge Research Laboratory

David Rank, Lick Observatory

Fred Witteborn, Ames Research Center

\section{OPTICAL AND ULTRAVIOLET}

\section{A S T RONOMY}

R. E. Danielson, Princeton University, Group Leader

William A. Baum, Lowell Observatory

Robert C. Bless, University of Wisconsin

G. Courtes, Laboratoire d'Astronomie Spatiale, France

A. Gaide, Observatoire de Geneve, Switzerland

C. R. O'Dell, Marshall Space Flight Center

Harlan J. Smith, University of Texas, Austin

\section{SOLAR PHYSICS}

John T. Jefferies, University of Hawaii, Group Leader

Loren W. Acton, Lockheed Palo Alto Research Laboratory

R. Bonnet, Laboratoire de Physique Stellaire, France

Carole Jordan, SRC Astrophysics Research Division, Culham Laboratory, England

Gordon Newkirk, Jr., High Altitude Observatory

Robert Noyes, Center for Astrophysics

Arthur B. C. Walker, Jr., Aerospace Corporation

Harold Zirin, California Institute of Technology

\section{I F E S C I E N C E S}

Robert F. Forster, University of Pennsylvania, Group Leader

Elso S. Barghoorn, Harvard University

Norman I. Bishop, Oregon State University

Neal S. Bricker, Albert Einstein College of Medicine

William H. Crosby, Scripps Clinic \& Research Foundation

Harold S. Ginsberg, Columbia University

Douglas Grahn, U.S. Atomic Energy Commission

Edward H. Kass, Boston City Hospital 
Patricia J. Lindop, The Medical College of St. Bartholomew's Hospital, London Irving F. Miller, University of Illinois at Chicago Circle

Mary J. Osborn, University of Connecticuî

John T. Shepherd, The Mayo Foundation

Patrick Winston, Massachusetts Institute of Technology

\section{PLANETARY EXPLORATION}

Michael B. McElroy, Harvard University, Group Leader

Roman Smoluchowski, Princeton University

\section{ESRO Attendees}

J. de Waard J. Ortner

\section{Other NASA Attendees}

Jospeh P. Allen

Anthony J. Calio

J. Allen Crocker

Philip E. Culbertson

Rufus R. Hessberg

Otha C. Jean

Douglas R. Lord

Leslie H. Meredith

John E. Naugle

NAS- NAE Staff

Space Science Board

David H. Garber

Ann Grahn

Bruce N. Gregory

Dean P. Kastel
Homer E. Newell

Goetz K. Oertel

Albert G. Opp

Nancy G. Roman

Alois W. Schardt

Erwin R. Schmerling

Gerald W. Sharp

Henry J. Smith
Aeronautics \& Space Engineering Board

John P. Taylor

LaRae L. Teel
Space Applications Board

Clotaire Wood 


\section{Foreword}

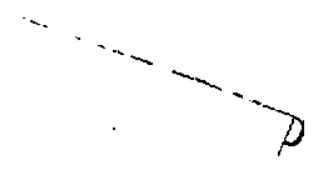

This is the report of a study convened by the Space Science Board of the National Academy of Sciences to explore the scientific uses of the Space Shuttle. The effort was focused on those aspects of the Shuttle most different from conventional launch-vehicle capabilities. In particular, the study considered the sortie mode, in which the Shuttle carries into orbit a payload that remains attached to the Shuttle and then returns to earth with the payload after one to four weeks. The study also considered the use of the Shuttle for launching, servicing, and recovering satellites and for launching lunar, planetary, and interplanetary missions.

Interest in the sortie mode is particularly great because of the recent decision by several European countries to develop a space laboratory (Spacelab) consisting of a pressurized module and an unpressurized platform called a pallet. Some combination of pressurized and pallet segments would be carried in the Shuttle bay on many sortie missions. The National Aeronautics and Space Administration (NASA) and the European Space Research Organization (ESRO) looked to the summer study to provide an understanding of the scientific requirements that might affect the design of the Shuttle and Spacelab.

Underlying the study was the premise that the Shuttle and Spacelab have been approved for development by national policy; therefore, in an approach recommended by NASA and adopted by the Academy, the study dealt with the use of the Shuttle and Spacelab for science and assumed that they will be developed. In a previous Space Science Board study* the Shuttle was considered in relation to its utilization for science; that study did not ". . . evaluate

*Priorities for Space Research 1971-1980, Report of a Study by the Space Science Board (National Academy of Sciences, Washington, D.C., 1971). 
the economics of the shuttle, because it depends so strongly on the volume of space traffic, which in turn is dependent upon many user activities besides... [science]."

Fifty U.S. and eleven European scientists participated in the study, which was held at Woods Hole, Massachusetts, during the first two weeks of July 1973. The following discipline areas were involved: atmospheric and space physics, high-energy astrophysics, optical and ultraviolet astronomy, infrared and radio astronomy, solar physics, iife sciences, and lunar and planetary exploration. Selection of these specific sectors of science was made to keep the scope of the effort manageable, and omission of another sector should not be construed as a judgment of its importance for the space program. Areas were most often omitted because they did not appear to impose significant specialized demands on Shuttle capabilities in the period before 1985 .

The study placed no constraints based on anticipated resources available on the programs under consideration. This was done so that imaginative proposals would not be eliminated on the basis of the limited cost information available at the time of the study. As a consequence, several of the conclusions, particularly the first two (see Chapter 1, Summary of Findings), depend heavily on a substantial scale of operations and level of funding for the space program.

Several reviewers have asserted that the scientific program outlined in this report is closer to the mainstream of fundamental advances in the physical sciences than in the life sciences. The Board recognizes that some scientific disciplines are substantially more dependent than others on space research; the life sciences are perhaps among the least dependent on space for the development of new concept. Exobiology (not taken up in this report) is a major exception to this view as it almost by definition presumes space exploration. Similarly, the vital areas of physiological and behavioral functioning in space, although dependent largely on terrestrial data, need extensive study in space to assure optimal safety and function of Shuttle and future crews. Knowledge derived therefrom can contribute in turn to terrestrial life sciences and life-sciences technology. The possibility that fundamental insights in biology and medicine can be gained in space should not be foreclosed.

The optimal use for science of an innovative space transportation concept is an exceedingly complex issue that can best be approached by stages. As Shuttle costs and capabilities become better defined,

$$
\text { viri }
$$


problems of scientific priorities will arise both within and among disciplinary areas. It is important to emphasize that the study does not explicitly address priority issues. Opinions about priorities are implicit in some of the statements by disciplinary groups, but these issues are subject to substantial study and possible revision in a future review of priorities, and in any case the implicit opinions do not necessarily represent the view of the Space Science Board at this time.

The Space Science Board is grateful to John W. Findlay for his contributions as Chairman of the study and to the participants for their efforts. The participants had the benefit of the presence of members of the NASA and ESRO staffs, who provided detailed information on Shuttle and Spacelab planning; their assistance is warmly appreciated. Thanks are due to the study director, Bruce N. Gregory, for his work with the study and its report. The Board acknowledges with appreciation the support of the National Aeronautics and Space Administration, particularly for Contract No. NASW 2509, which made this study possible.

Richard M. Goody, Chairman Space Science Board 


\section{Preface}

Considerable supporting work had been accomplished within the United States and Europe before the Space Science Board's study on scientific uses of the Space Shuttle began. The 1973 Woods Hole meeting was timed to make best use of this earlier work and to provide a useful input to the European Spacelab design studies.

By the start of the study, engineering definition of the Shuttle and Spacelab had proceeded to the stage where the most important performance characteristics were known. The participants had briefing documents and the continuous availability of members of the NASA and ESRO staffs to elaborate and explain planned performance and operational details.

Study of the scientific usefulness of the Shuttle had been in progress since a NASA workshop was convened in August 1972 at Goddard Space Flight Center to study the sortie mode. The report of this workshop was published in August 1972.* Studies were continued and expanded involving European scientists and U.S. scientists from outside NASA. The results of this work were published in May 1973 as ten discipline-group reports and a summary volume. $\dagger$ Volumes 1 through 5 formed a starting point for the work of the 1973 Woods Hole study.

Similar efforts had been conducted in Europe, under the leadership of ESRO. A symposium to report progress was held in Frascati, Italy, in January 1973, and the final study report was published in May 1973. ${ }^{\ddagger}$

*Proceedings of the Space Shuttle Sortie Workshop. Volume I, Policy \& System Characteristics; Volume II, Working Group Reports (NASA Goddard, Space Flight Center, Greenbelt, Md., 1972).

$\dagger$ Final Reports of the Space Shuttle Payload Planning Working Groups, Volumes 1-5 (NASA Goddard Space Flight Center, Greenbelt, Md., 1973).

†Spacelab Programme; Views of the ESRO Spacelab Payload GroupsUtilisation of the Spacelab for Science (ESRO, Neuiliy-sur-Seine, France, 1973). 
These NASA and ESRO reports were the basic background material for the Academy study, and some of the scientists who had contributed to the earlier efforts were at Woods Hole. However, the NASA and ESRO reports were not intended to be definitive, and participants at the study were not bound by their conclusions.

At Woods Hole, the discipline groups were asked to describe the scientific objectives of their respective disciplines; to identify experiments or instruments that are both scientifically desirable and suitable for Shuttie operations; to determine which mode of Shuttle use would be best suited to the operation of these instruments; to outline a mission model; and to make recommendations concerning their science and the Shuttle. All groups found the first two requests easy to meet. In choosing the best-suited Shuttle mode, almost all were limited by the lack of detailed information on costs and, hence, on the cost-effectiveness of various modes. Similarly, in formulating mission models for the 1980's, the question of cost remains to be defined.

On the first day of the study, participants were briefed by NASA and ESRO on relevant information about the Shuttle and Spacelab concepts. The rest of the first week was given to meetings of the discipline groups; a steering committee, including the discipline group leaders, met on most mornings to monitor progress. The first week concluded with a half-day reporting session to the whole study. A working group, composed of members from all disciplines and from the steering committee, was formed at the start of the second week to focus on the scientific requirements for the sortie mode and free-flying spacecraft. This group (whose work is reported in Chapter 2) worked closely with the discipline groups, which continued to work until the study ended. After a final plenary session, the study made an oral report to NASA and ESRO on July 14.

Summer studies are always hectic events, and some of the greatest demands are placed on the secretarial staff; the participants are grateful for the unfailing efforts of Lally Anne Anderson, Jo Ann Severance, and Barbara White. Kathleen Davison and Mildred McGuire typed the manuscript. I am grateful to all those who participated in the study for their hard work and cooperative efforts.

John W. Findlay, Chairman

Scientific Uses of the Space Shuttle 


\section{Contents}

1. SUMMARY OF FINDINGS 1

I. SUMMARY 1

II. THE ROLE OF MAN 3

III. SIZE OF THE PROGRAM 3

IV. LOWERING THE COST OF SCIENCE IN SPACE 4

2. MODES OF SHUTTLE USE

I. ASSUMPTIONS AND OBJECTIVES 5

II. GENERAL CONCLUSIONS 8

III. PALLET MODE OF OPERATION 9

IV. SHUTTLE-LAUNCHED FREE-FLYERS 10

V. ROLE OF MAN 11

A. General-Purpose Payload Specialist Station 11

B. Utilization of the Pressurized Module 12

C. Ground Experiment Operation 13

3. ATMOSPHERIC AND SPACE PHYSICS

I. INTRODUCTION 14

II. ATMOSPHERIC SCIENCE 14

A. Our Understanding of the Upper Atmosphere by $1980 \quad 14$

B. Need for the Shuttle 15

C. Thermal Structure and Dynamics 17

D. Neutral-Atmosphere Chemistry below $120 \mathrm{~km} \quad 18$

E. Ion Chemistry of the D-Region and Lower E-Region 20

III. MAGNETOSPHERIC DYNAMICS 21

A. Background 21

B. Major Magnetospheric Physics Problems of the 1980's 25

IV. PLASMA PHYSICS IN SPACE 27
A. Background 27
B. Outstanding Problems 28

V. INSTRUMENTS AND TECHNIQUES 30

A. Atmospheric Science 30

B. Space Plasma and Magnetospheric Physics 32

VI. MISSION MODEL FOR ATMOSPHERIC AND SPACE PHYSICS

VII. RECOMMENDATIONS 37 


\section{HIGH-ENERGY ASTROPHYSICS}

I. INTRODUCTION 40

II. SCIENTIFIC OBJECTIVES 41
A. X-Ray Astronomy 41
B. Gamma-Ray Astronomy
C. Cosmic-Ray Astronomy
48

III. INSTRUMENTS 51
A. Achievement of Objectives
B. X-Ray Instruments for the Shuttle Era
C. Gamma-Ray Experimental Program 63
D. Cosmic Rays 70
E. Shuttle Sortie Mode Requirements $\quad 74$

IV. PROGRAM IMPLEMENTATION 76
A. Single-Investigator Experiments 76
B. National Facilities 78

V. MISSION MODEL 79
A. Automated Program
79
B. Sortie Mode 80

VI. SUMMARY AND RECOMMENDATIONS

\section{INFRARED ASTRONOMY}

I. INTRODUCTION 83

II. SCIENTIFIC OBJECTIVES
A. Solar-System Formation
84
B. Stellar Evolution 84
C. Galactic Structure and Evolution 84
D. Cosmology 85

III. SHUTTLE TELESCOPES: EVOLUTIONARY APPROACH
A. Cryogenically Cooled Telescopes 86
B. Ambient-Temperature Telescopes 89

IV. SPECIALIZED INSTRUMENTS
A. Cosmic Background Radiation 92
B. Discrete-Source Sky Survey, Infrared Monitor
92
C. Spatial Interferometer 93

V. REQUIREMENTS ON THE SHUTTLE CAPABILITY
A. Sortie Mode 93
B. Launch Mode 96
C. Assembly Mode 96
D. Contamination 96

VI. POTENTIAL MISSION MODEL $\quad 98$

VII. SUPPORTING RESEARCH AND TECHNOLOGY 98

VIII. SUMMARY AND RECOMMENDATIONS 100

\section{OPTICAL AND ULTRAVIOLET ASTRONOMY}

I. SCIENTIFIC OBJECTIVES 102
A. Solar System
102
B. Stars and Stellar Systems
103
C. Interstellar Matter
104 
D. Emission Nebulosities 105

E. Galactic Nuclei and Quasars 106

F. Intergalactic Matter 106

G. Extragalactic Research 107

H. Cosmology 108

II. CANDIDATE SHUTTLE-LAUNCHED INSTRUMENTS 108
A. The Large Space Telescope 108
B. Diffraction-Limited Telescope 111
C. Small General-Purpose Telescope 112
D. Very-Wide-Field Survey Camera 113
E. Very Large Light Collector 114
F. Other Instruments 115

III. TECHNICAL IMPACT ON SHUTTLE 120

A. The Role of Man in Space-Shuttle Astronomy 120

B. Pointing and Stabilization 121

C. Contamination 122

D. Thermal Requirements 123

E. Orbits 123

F. Payload Weight 124

G. Detectors and Telemetry Requirements 124

IV. MISSION MODEL 125

V. SUMMARY AND RECOMMENDATIONS 126
A. Utilizing the Shuttle for Optical and Ultraviolet Space Astronomy 126
B. Supporting Research and Technology

129

7. SOLAR PHYSICS

1. SOLAR-PHYSICS OBJECTIVES AND OVERALL PLAN 130
A. Solar Activity 130
B. Energy and Mass Flow in the Solar Atmosphere
C. Physical Problems of Broader Significance 133
D. Relation of Solar Physics to Other Disciplines 134

II. PROFILE FOR A BALANCED PROGRAM IN SOLAR ASTRONOMY 135

A. Spaceflight Aspects 135

B. Other Necessary Components of a Balanced Program

III. MISSION MODEL 137
A. The Pre-Shuttle Solar Maximum Mission $\quad 138$
B. Use of the Space Shuttle for Solar Research 140

IV. REQUIREMENTS IMPOSED ON SHUTTLE AND

SPACELAB BY THE SOLAR PROGRAM 146
A. Contamination of the Optical Environment
146
B. Scheduling of Solar Missions
147
C. Orbital Considerations
147
D. Tracking and Data-Relay Satellite System (TDRS)
E. Payload Capacity 147
F. Mission Duration 148
G. Use of the Payload Specialist Station $\quad 148$
H. Data and Control Interfacing 148 


\section{$x \cdot v$}

V. GENERAL CONSIDERATIONS 149
A. The Impact of Quality Assurance on Costs
D. Convening of a Shuttle Expeciinineintatitioui Planning Committee 149
C. Selection and Responsibilities of Scientists
149
D. The Crucial Role of SR\&T Support 150

VI. RECOMMENDATIONS 150

\section{APPENDIX A 152}

1. Representative Focal Plane Instrumentation for Use with the Solar Telescope Cluster 152

2. Representative Special-Purpose Instruments for a Large Fine-Pointed Platform 153

3. Representative Instruments for a Small Fine-Pointed Platform 153

4. Representative Coarse-Pointed, High-Energy Instrumentation 153

5. Representative Instruments for a Large Fine-Pointed Platform 153

8. LIFE SCIENCES

I. INTRODUCTION 154

II. CELLULAR AND MOLECULAR BIOLOGY 155

III. ORGANISMIC BIOLOGY 157
A. Plant Biology 157
B. Animal Biology 159

IV. BIOMEDICINE 160
A. Cardiovascular System 161
B. Respiration 162
C. Kidney and Metabolism 162
D. Hematology 163
E. Neurology 163
F. Microbiology 164

V. BEHAVIOR 165

VI. RADIOBIOLOGY 166

VII. LIFE-SUPPORT TECHNOLOGY 168

VIII. LABORATORY OPERATIONS 171

IX. EXPERIMENTAL AND ADMINISTRATIVE APPROACHES 174

$X$ RECOMMENDATIONS 175

\section{PLANETARY EXPLORATION}

I. OBJECTIVES 179

II. THE INNER PLANETS 181

III. THE MOON 182

IV. THE OUTER PLANETS 184

V. PLANETARY SPACECRAFT 187

VI. PROPULSION REQUIREMENTS 190

VII. RECOMMENDATIONS 195 


\section{1 \\ Summary of Findings}

\section{S U M M A R Y}

1. The Shuttle can be an important asset to scientific research in and beyond the 1980's. All discipline groups in the study found aspects of the Shuttle capability important to their science. Each made specific recommendations in its report about scientific needs and, in some cases, about the Shuttle characteristics and modes of use.

2. An important aspect of the Shuttle system for science will be its ability to carry many large and heavy payloads into orbit with potentially substantial economies. Of all the changes that the Shuttle may bring to space science, the increased size and weight of the payloads that can be orbited with the possibility of reducing costs by simplifying design and construction and the possibility of a high rate of launch were singled out.

3. Many of the potential advantages of the Shuttle depend on the development of efficient and flexible procedures for flying multipurpose missions and combined payloads. Most discipline groups found a need for Shuttle missions entirely dedicated to their own science but also recognized the potential savings associated with missions with multiple objectives. The study group identified some problems of instrument design and integration and, to some extent, the kinds of operational procedures that will be needed for multipurpose missions. This finding drew attention to the need for simplifying as far as possible all the steps that lie between the first concept of a space experiment and its eventual flight as a part of the Shuttle payload.

4. The ability of the Shuttle system to recover or service payloads in orbit will be of special value for large and expensive systems such as large observatories; for some less expensive payloads the economic advantages of recovery and of possible servicing are unclear. The 
importance of recovery and service of payloads placed in orbit is frequently emphasized. However, if smaller, less expensive orbiting spacecraft are considered, advantages are less clear; it may be that incompatibility of Shuttle and spacecraft orbits will make visits too costly. Limits on the weight of the payload that can be returned from orbit may also restrict recovery. The economics of payload recovery and servicing must be studied further.

5. Most planetary missions can be launched with a Shuttle/ Centaur system. Some missions identified for the 1980's require additional capabilities such as might be provided by Tug, solar electric, or some other advanced propulsion system.

6. For biomedical research in space, the study identified a clear and essential requirement for the use of the manned pressurized space laboratory.

7. Many disciplines require rapid interaction between man and payload. This function appears to be adequately fulfilled in many cases by the payload specialist and his console. However, for some experiments in atmospheric or space physics in which continuous involvement of man is required, the pressurized space laboratory is highly desirable. The need for man is present, to some extent, in all disciplines. In high-energy astrophysics it is perhaps the smallest, and in biomedical research the greatest. It was the opinion of many study participants that the presence of a payload specialist in the Shuttle orbiter could serve their needs. However, this depends on the amount of his time available and on the degree to which it is possible to use the payload specialist's console as an experiment control center. For some experiments it is possible to have scientists either in a space laboratory or on the ground, linked with the payload by a high-data-rate real-time system. The latter implies the existence of capabilities similar to those suggested for the proposed independent Tracking and Data Relay Satellite (TDRS). The study participants realized that such a TDRS is only in the planning stage and see the need for further work to clarify how realistic this option is.

8. The ability to operate instruments mounted in the Shuttle bay (in the pallet mode, with or without a pressurized laboratory) is an important feature for all disciplines except the life sciences.

9. Payloads carried into orbit by the Shuttle and then released as free-flyers are major elements in most discipline programs. Most disciplines identified major programs requiring observing times considerably in excess of the 28-day maximum duration envisaged 
for sortie missions; the most cost-effective way of carrying out such programs is by using free-flying automated spacecraft.

10. For most discipline groups, the 28-day sortie mission duration (or even longer if possible) is judged to be very valuable.

\section{THE ROLE OF MAN}

One of the central concerns of the summer study was to explore the nature of the role that man will play in Shuttle-related science. One problem centers around the weight of the Spacelab needed to provide working space for men in addition to the crew; this weight may well place limits on the scientific payload that can be carried on some missions. In addition, the one- to four-week duration of manned Shuttle missions is considerably less than the time that many scientific programs require, leading to the belief that these programs could best be accommodated by unmanned free-flyers.

Scientific interest in the Spacelab is greatest for the life sciences, which require man to work in the shirtsleeve environment of a Spacelab module. Other disciplines, if they require a pressurized module at all, would use a smaller module than would the life sciences. The weight penalties of carrying a Spacelab raise the question of whether real-time control and evaluation would be better supplied from a ground-based scientific group or from scientists carried in flight. If a communications system from the Shuttle to the ground, giving continuous global coverage with a high rate of data interchange, were available, then ground control might be preferable to carrying a manned Spacelab. This question requires further detailed study.

\section{SIZE OF THE PROGRAM}

The overall scale of Shuttle space science and the proportions of Shuttle opportunities that will go to various scientific disciplines can only be established when a realistic model of Shuttle operations becomes clearer. This model will, of course, depend very much on the funding available for space science and applications during and following the development of the Shuttle. Initial planning for Shuttle science will bring to the forefront priority choices-choices that must be faced in the near future. We can see the need for a significant effort in supporting research and technology to begin the development of payloads for Shuttle missions. 


\section{LOWERING THE COST OF SCIENCE I N S P A C E}

There is considerable hope that Shuttle-borne science will be less expensive and easier to fly than conventionally launched space science. In order to lower the cost of Shuttle science, a design and management philosophy must be instituted that provides maximum scientific flexibility and minimum restrictions and documentation. Many of the steps to be taken are clear for payloads that remain attached to the Shuttle throughout the missions. Mounting, pointing, and other systems can be developed in a single form to serve many purposes. For systems that are common to several different experimental packages, it should be possible to develop commercial units that are qualified for Shuttle use. It is crucial to set design and test criteria for flight hardware and to use a management system similar to those used for rocket-launched payloads, which are low in cost compared with satellite payloads.

The reduction of overall costs in payloads that are separated from the Shuttle is a more complex question that requires detailed cost-effectiveness studies; the reduction of weight and size limitations may permit significant savings. Studies have suggested that savings will come from recovering and refurbishing satellites. This might be true for military and possibly commercial satellites, but the situation is less certain for scientific satellites. A sophisticated Large Space Telescope might be worth the cost to revisit and service or to return to earth, but this approach is less attractive for less expensive free-flying payloads. The costs of revisits and potential economies depend closely on the overall Shuttle flight pattern and the compatibility between the orbits needed for free-flying scientific experiments and those that the Shuttle will use for entirely different missions. On the latter missions, the Shuttle might have capability to revisit or recover the free-flyers at little additional cost. Such questions must be answered before the choice of the least expensive and most effective ways of flying scientific missions can be ascertained.

In summary, the study group found that the Shuttle will have many capabilities potentially of great value to space science. It will provide opportunities sufficiently different from the ways in which we now conduct space science that they demand innovations in management and execution if we are to minimize costs and realize the full benefits of this new space transportation system. 


\section{2 \\ Modes of Shuttle Use}

\section{ASSUMPTIONS AND OBJECTIVES}

An interdisciplinary group was convened to identify major demands placed on the Shuttle, particularly by the sortie mode. The group considered experiments carried to near-earth orbit by Shuttle, either (1) in pressurized modules; (2) on unpressurized 3-m pallet elements; or (3) on free-flying spacecraft, initially mounted to, but eventually ejected from, 3-m pallet elements. Deep-space planetary missions were excluded from these considerations, as was the Large Space Telescope.

The group identified ten different modes under which a potential experiment could be performed. These modes are summarized in Table 1. The discipline groups were invited to propose representative experiments, using a simple proposal form, a copy of which is shown in Figure 1. Some 70 experiments were proposed and were used by the group to identify major demands on the sortie mode. The general conclusions summarized in Section II were based on these experiment proposals.

No attempt was made to identify priorities. Considerations that led to specific choices of experiment mode were not explored in any depth; it was assumed that the choices were made after proper consideration of alternatives within the various discipline groups. The critical elements in these deliberations were instrument weight and the role of man; fiscal considerations appear to have entered only peripherally. In a comprehensive analysis, the requirements for a human operator should be evaluated against the availability of Shuttle-furnished communication facilities and communication satellites; possible simplifications in experimental design as a result of manned operation should be judged against the weight penalties of necessary human-support systems. Due to limitations in time, these 
TABLE 1 Possible Experiment Modes
A. A payload that remains on the pallet throughout a flight, using orbiter communications
B. A payload that remains on the pallet throughout a flight, using its own communications direct to earth
C. A payload that remains in the pressurized Spacelab throughout a flight
D. A payload taken to orbit, ejected from the orbiter, used as an automated free-flyer and never visited nor recovered
E. As $D$, but visited and not recovered
F. As D, but visited and recovered
G. As D, but recovered with the same Shuttle flight that ejected the payload
H. A payload taken to orbit to replace an essentially identical spacecraft already in orbit
I. Instrumentation deployed from orbiter on booms or similar devices, outside payload bay
J. Instrumentation requiring orbital assembly, e.g., a very large telescope

tradeoffs were addressed only tangentially in many cases. A more definitive study would require assigning priorities to the various experiments and a more careful fiscal analysis of available experiment options.

Detailed requirements for specific experiments were identified using an interrogation form, shown in Table 2. This information was made available to NASA and ESRO personnel and is expected to aid in future design of the sortie mode. It should be possible with these data to identify generally useful support systems that could be

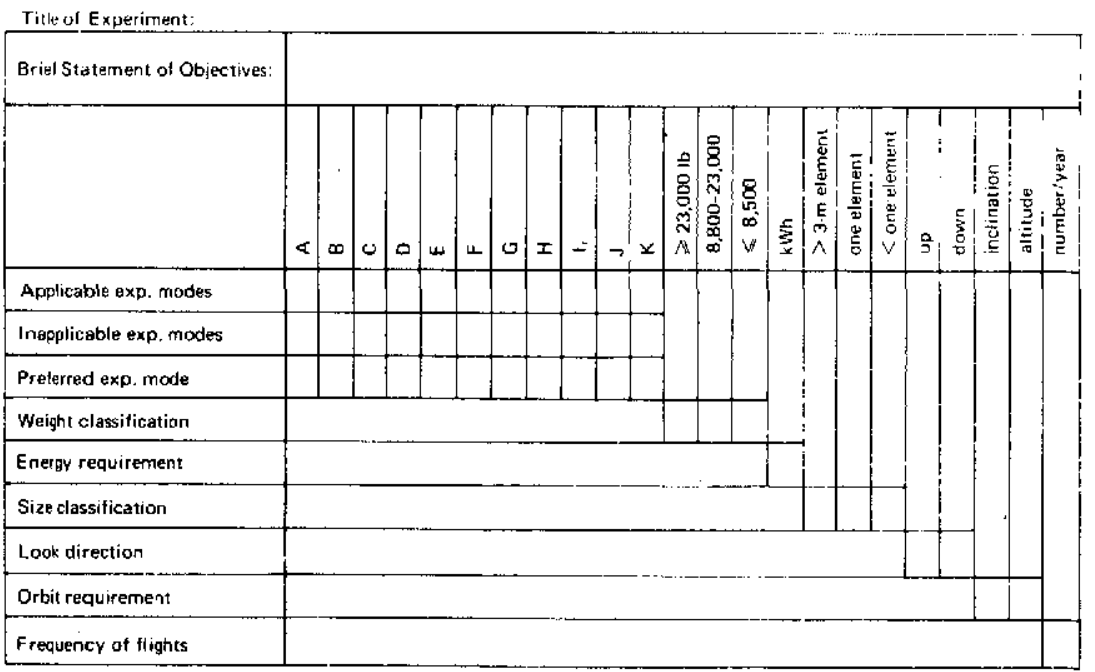

FIGURE 1 Experiment proposal form. 
TABLE 2 Sortie Mode Requirements

\section{EXPERIMENT TITLE}

1. Role and No. of Men
a. \% Time Demand on Shuttle Payload Support Crew
b. Extra Specialists in Orbiter Cabin
c. Extra Specialists in Lab Module
d. Other

2. Shuttle Flights and Requirements
a. Inclination
b. Attitudes on Orbit
c. Altitudes
d. Number of Total Flights
e. Rate of Flight
f. Duration in Orbit
g. Other

3. Volume and Weight of Instrumentation
a. In Lab Module
b. In Pallet Area
c. In Orbiter Cabin
d. For Deployment on Booms Outside Payload Bay
e. Free-Flyer(s)
f. Other

4. Support Requirements
a. Power Level and Total Energy
b. Pointing (1) General Direction
(2) Stabilization Limits
c. Data: Storage
d. Communications (1) To Earth
e. Thermal Control
(2) To Shuttle
f. Booms, Special Tools, Airlocks, and Windows
g. Visual or Access to Payload
h. Other

5. Special Considerations
a. Contamination
b. Noise and Vibration
c. Hazardous Materials or Procedures
d. Cryogenics
e. Radiation Belt Concerns
f. Other

6. Schedule Considerations
a. For a Given Flight (Date Windows)
b. Minimum Lead for Specific Flight
c. Other 
incorporated into the pressurized module and basic pallet elements. Use of standard power, energy, pointing, and communications equipment could lead to major cost economies and conveniences for potential sortie mode experimenters.

\section{GENERAL CONCLUSIONS}

Study of the various experiment proposals led us to some general conclusions:

1. There is a general desire for manned interaction in real time with experiments. The demands are most strongly stated by life scientists, space physicists, and solar astronomers. The manned interaction can occur in several ways: either with man in the Shuttle cabin, in the pressurized module, or on the ground. The merits of these various possibilities are discussed below. Pressurized modules are essential for some experiments in the life-science area.

2. A major fraction of all experiments proposed can be implemented using unpressurized 3-m pallets.

3. A significant number of experiments, especially in the area of high-energy astronomy, require extensive observing time and are best implemented using free-flying spacecraft.

4. There is a clear need for a design and management philosophy that will allow for maximum flexibility and that will minimize difficulties and costs associated with spaceflights.

5. Certain natural events of great scientific interest are infrequent and short-lived. Typical examples are outbursts of supernovae, novae, flare stars, solar flares, and geomagnetic storms. Dedicated instruments, perhaps with dedicated pallets, should be held in standby status for such opportunities. The logistics of Shuttle operations should permit inclusion of such payloads, prefcrably in the next scheduled Shuttle launch, even if bumping of another payload is required. Guidance for such emergency programming should come from a rating of scientific priorities.

6. It is extremely important to provide the opportunity to fly small experiments on a cost-effective basis. We believe that this can be accomplished by providing small standardized pallet elements (a pointed element and an unpointed element, each one half standard element in size, for example) on which experiments can be integrated and placed in a standby status until a Shuttle mission able to carry additional weight can be identified. The small elements could then be fitted into Shuttle flights on a space-available basis. If this concept is 
to be carried out in a cost-effective way, considerable attention should be paid to the management of this type of opportunity. We believe that the present rocket and balloon programs should serve as models for this mode of operation. Careful consideration should be given to make the standardized pallets as self-sufficient as possible and to make the interface of these pallets with the orbiter or sortie lab as simple as possible, in order to maximize opportunities for flight.

7. There is a general recognition of the potential importance of the mission specialist. The mission specialist should play a role analogous to the mission manager on NASA-Ames airborne science flights, to the Los Alamos physicist working with a team of visiting physicists running on the Meson Facility, or to the science coordinator on board an oceanographic research ship. He should be a facility person familiar with facility requirements but also familiar with the needs and scientific objectives of the user groups.

\section{PALLET MODE OF OPERATION}

It is important to distinguish at least two major kinds of pallet operation. The first is a pallet-only mode in which the entire Shuttle payload is an integrated set of pallet sections. The second consists of a given set of experiments contained on a single pallet section. In both of these operations the experiments best suited for flight are those whose objectives can be met in a relatively short time, that is, from a week to several weeks.

For a pallet-only mission, the experiment control may be from the orbiter cabin, at the Payload Specialist's station, or directly from the ground through available telemetry links. For essentially continuous control and operation of this kind of payload, it may be necessary to have the use of a data relay satellite.

It is expected that the common support equipment needed for these experiments would be provided by the pallet, drawing perhaps on the orbiter facilities as needed. These common facilities include power, up and down telemetry, thermal control, data handling, and such specialized equipment as pointing platforms. In this instance, each separate pallet module would receive these services from a common source, and the entire unit (set of modules) would be integrated prior to delivery for launch. Such a mission could be discipline-dedicated or multidisciplinary in nature. It should be recognized that the integration problem for multidisciplinary missions may be significant. 
A single-pallet-element mission could be flown attached and integrated to other pallets or simply added on to a nonpallet mission (i.e., on some Tug or large free-flyer delivery mission). Single elements have the advantage that they may be fully dedicated to a particular investigation and may be more accessible to the experimenter during experiment installation and checkout. Further, it should be possible to obtain more frequent flight opportunities for individual elements than for a full pallet payload.

To meet the desires of the users, this pallet section (or module) should be as autonomous as is practical. That is, it should contain its own power conditioning unit, data-handling system, thermal control, and pointing controls. It may draw upon the Shuttle system for raw power and utilize the Shuttle telemetry system.

Where high data rates on a nearly continuous real-time basis are required, a data relay satellite may be required. The present Shuttle design does not include a steerable high-gain antenna that would permit a high-data-rate transmission through the relay satellite. Thus pallets or pallet sections may have to include their own steerable antenna $(1-\mathrm{m})$ to make this possible.

\section{SHUTTLE-LAUNCHED FREE-FLYERS}

Many scientific objectives can be met only by the use of free-flying satellites. These include (a) missions that require observation time substantially longer than the maximum of 30 days provided by the Shuttle itself, (b) missions that have a need for higher altitude orbits than can be obtained from the Shuttle, and (c) missions that include experiments requiring substantially smaller contamination than the Shuttle payload bay can provide.

Such free-flyers may well be pallet-sized and could be launched from a pallet in the payload bay. A very significant feature of these is that they may be retrieved by the Shuttle and refurbished for subsequent redeployment, should that prove to be economically and scientifically desirable.

It is possible that some kind of standard free-flyer should be designed to reduce costs-particularly for missions that can be performed in a Shuttle-compatible orbit-and to facilitate retrieval and refurbishment of the satellite. An attractive feature of the Shuttle-compatible free-flyer is the possibility of recalibrating instruments after they are returned to the ground. 


\section{ROLE OF MAN}

\section{A. General-Purpose Payload Specialist Station}

A standard interface can be established between the specialist station and the payload by using a digital computer-to-computer interface, switches to operate standard relays in the payload, and selsyn-toselsyn controls for interfaces requiring analogue control. A station with the following capabilities could serve a multitude of experimental requirements:

1. Display computer with two-color TV screens (one used for status information, the other for data display). A camera is required for recording selected displays, and pictures should be available immediately.

2. Teletype keyboard for controlling displays and for commanding the experiment computer. (Tape input would be desirable.)

3. Digital displays of key control parameters (ten might be adequate).

4. A set of switches to operate standard relays in the payload.

5. Analogue controls (probably using selsyns) to operate an equivalent control in the payload. It would be desirable to have a number of simple selsyns (about ten) and two joystick-type controls for two dimensions.

6. TV monitor display.

The control console would interface through standard connectors with the control package mounted on the pallet. The control part of this package is the experiment computer that controls most of the functions in the experiments and records the experimental data. This computer is reprogrammable from the ground and from the payload specialist station. It has access to on-board data storage and to the telemetry readout. Substantial on-line memory ( $>128 \mathrm{kbits}$ ) and mass data storage should be included in the computer's capabilities.

As required, this control package would have digital channels for special displays on the console. The package could also contain selsyns that control the experiments directly or control the circuit that generates the driving voltages required by the experiment.

Prior to integration into the Shuttle, the performance of the pallet payload would be checked out with a copy of the payload specialist station. The interfaces would be standard and have a margin for 
variability between units so that identical performance can be expected after the pallet is mounted on the Shuttle.

\section{B. Utilization of the Pressurized Module}

The pressurized module with its closed and controlled atmosphere is clearly necessary for the primary life-sciences missions, as described in the report of that working group. However, use of a smaller pressurized module can provide very significant benefits for other scientific disciplines as well. In several areas it is vital that real-time control of the experiments be carried out on the basis of real-time evaluation of observations. Some very complex coordinated palletmounted instrumentation facilities are under consideration, and in these cases, the general-purpose payload specialist console may not provide an adequately flexible facility for display and control of the large number of unique parameters of importance for the mission. For example, to carry out an experiment that requires deployment of both a boom package and a maneuverable subsatellite in changing sequences of directions, with known and varying sensor orientation, there must be an opportunity for continuous feedback between the output of any of the remote sensors and the control circuits.

If the presently planned Tracking and Data Relay Satellite (TDRS) system provides global and continuous coverage for all types of Shuttle missions, including high-inclination ones, and if the necessary extensive real-time ground data reduction and command facilities are provided, it is possible, in principle, to carry out this manned evaluation and control from a sophisticated central ground laboratory. However, there are certain additional important advantages associated with use of an on-board scientist in a small pressurized module.

The on-board scientist with access to the display and command console will be able to respond to unexpected situations by effecting limited repairs, by replacing redundant system elements, and by evaluating the stability of prelaunch calibrations. Moreover, there are potential hazards associated with deployment and recovery or retraction of subsatellites and booms, and it may be decided that on-board control provides greater safety for these operations than does dependence on a remote radio link. 


\section{Ground Experiment Operation}

Many sortie experiments can most effectively be operated from a ground terminal. This approach has the potential advantages of allowing the following:

1. Senior investigators and their staffs to conduct their observing programs directly;

2. Use of general-purpose instruments on the Shuttle by a number of different groups of investigators;

3. Application of relatively powerful ground computing and data-handling systems to the implementation of the experiment;

4. Experiment implementation to be conducted from a single location where all orbit, attitude, experiment telemetry data, and preflight calibration and test data reside;

5. All the experiment information to be available to the best trained experts in the case of anomalous experiment behavior;

6. Experiment operation to be conducted relatively independently of the Shuttle sortie on-board time line;

7. Operation of the sortie experiment in a mode similar to that of the free-flyers with which many investigators are trained.

In order to use a substantial class of Shuttle experiments effectively, it is necessary to have real-time data displays available on the ground, for the use of a team of scientists in making decisions on the modification of observing programs. This mode of operation is particularly important for the astronomical disciplines: ultraviolet, optical, and infrared astronomy; solar physics; and high-energy astrophysics. The presently planned data link through the TDRS system is inadequate in bandwidth, and we recommend that a wideband link be made a standard capability of the orbiter/sortie lab (pallet and module) configuration from the onset of Shuttle operations. A data rate of $256 \mathrm{kbps}$ continuously would meet most requirements. A few specialized requirements, such as observation of candidate black holes in high-energy astrophysics, require considerably higher rates (10 Mbps). These requirements can probably be met by the use of temporary storage on the pallet and subsequent playback through the TDRS system at a lower rate. 
3

\section{Atmospheric and Space Physics}

\section{I N T R O D U C T I O N}

Three fields of research are represented by the Working Group in Atmospheric and Space Physics: the atmosphere of the earth, the magnetosphere of the earth, and plasma physics in space. These fields will be able to make effective use of the Shuttle and Spacelab when they become available. Optical remote sensing of the mesosphere and stratosphere to study chemical composition, structure and dynamics, active plasma experiments, and controlled study of magnetospheric phenomena requires the payload capacity and flexibility offered by the Shuttle system. We foresee a need to make use of the sortie mode as well as free-flyers. Because many experiments require manned control in real time based on real-time analysis of observational data, the presence of scientists in a small pressurized module in the Spacelab is a preferred mode of operation: Because of the requirement for data acquisition and control in polar regions for some experiments, the pallet-only Spacelab mode will fulfill all of our needs only if an adequate data analysis and tracking system is provided.

\section{ATMOSPHERIC SCIENCE}

\section{A. Our Understanding of the Upper Atmosphere by 1980}

By 1980, the neutral upper atmosphere will have been the subject of over 20 years of extensive investigation using space vehicles. Much of this investigation has concentrated on the region above $250-\mathrm{km}$ altitude, where satellites can remain for prolonged periods in earth orbit. Reference atmospheres have been compiled that successfully describe the gross features of the structure, composition, and 
variabilities of this upper part of the atmosphere, although a number of discrepancies remain.

Further measurements are planned during the next several years using the Atmosphere Explorer AE-C, -D, and -E spacecraft, which will be equipped to measure simultaneously the neutral constituents, the incident photon flux, and the ionized constituents, down to an altitude of $120 \mathrm{~km}$. The region of $120-200 \mathrm{~km}$ altitude, where most of the extreme-ultraviolet solar photons are absorbed, has not been studied in situ by satellites before and is the region of greatest uncertainty in existing upper-atmosphere models.

With the completion of these missions, we expect that by 1980 the structure and photochemistry of the atmosphere above $120 \mathrm{~km}$ will be well understood. On the other hand, the relationship among the circulation of the thermosphere, the dynamo current system, and the magnetospheric electric field will not have been adequately studied. A follow-on Electrodynamics Explorer mission to investigate the magnetospheric electric field and its relationship to the largescale dynamics of the thermosphere has been suggested but not yet approved; such a mission would contribute substantially to this study.

By 1980 , the most important regions of the atmosphere still to be explored and understood will be the stratosphere, mesosphere, and lower thermosphere up to $120 \mathrm{~km}$. Circulation and the effects of transport on chemical composition will be òf particular interest. Recent concern about the effect of emissions from supersonic transport engines on the ozone layer demonstrates the importance of understanding this region of the atmosphere. At present, the lack of suitable sampling techniques and sensors limits us to only scattered and fragmentary data concerning the concentration of such important constituents as $\mathrm{O}_{3}, \mathrm{NO}_{x}, \mathrm{H}_{2} \mathrm{O}, \mathrm{H}_{2}$, and $\mathrm{HNO}_{3}$.

\section{B. Need for the Shuttle}

Our understanding of the upper stratosphere and mesosphere will be limited in the next few years because of the inherent difficulties associated with direct in situ rocketborne measurements, which provide only partial information on spatial and temporal variations. Optical remote sensing from the ground has limitations, and the complexity of the instrumentation required for comprehensive active and passive topside soundings is formidable. 
The meteorological satellites planned for the next few years have remote-sensing capabilities in the spectral region from ultraviolet through microwave to provide information on temperature and ozone and water-vapor distributions in the troposphere and stratosphere, but there are limitations in the capacity of these systems to probe higher altitudes and other constituents.

The instruments needed for active and passive remote sensing include sensitive, high-resolution scanning instruments and large tunable monostatic and bistatic laser radar (lidar) systems. The Shuttle will be able to accommodate the long focal lengths and large apertures of such instruments. The Shuttle will also be able to accommodate cryogenic systems to cool detectors and give vastly improved signal-to-noise ratios in some of the instruments. The Shuttle will provide for the first time the means to study adequately the distribution of the chemically active constituents of the middle atmosphere in space and time by remote sensing.

The interplay of optical radiations and chemical effects shown in Figure 2 is reasonably well understood, although the number of significant neutral and ion species involved is large. However, the dynamical properties and the ways in which they interact with the structure and chemistry are understood only in very general terms.

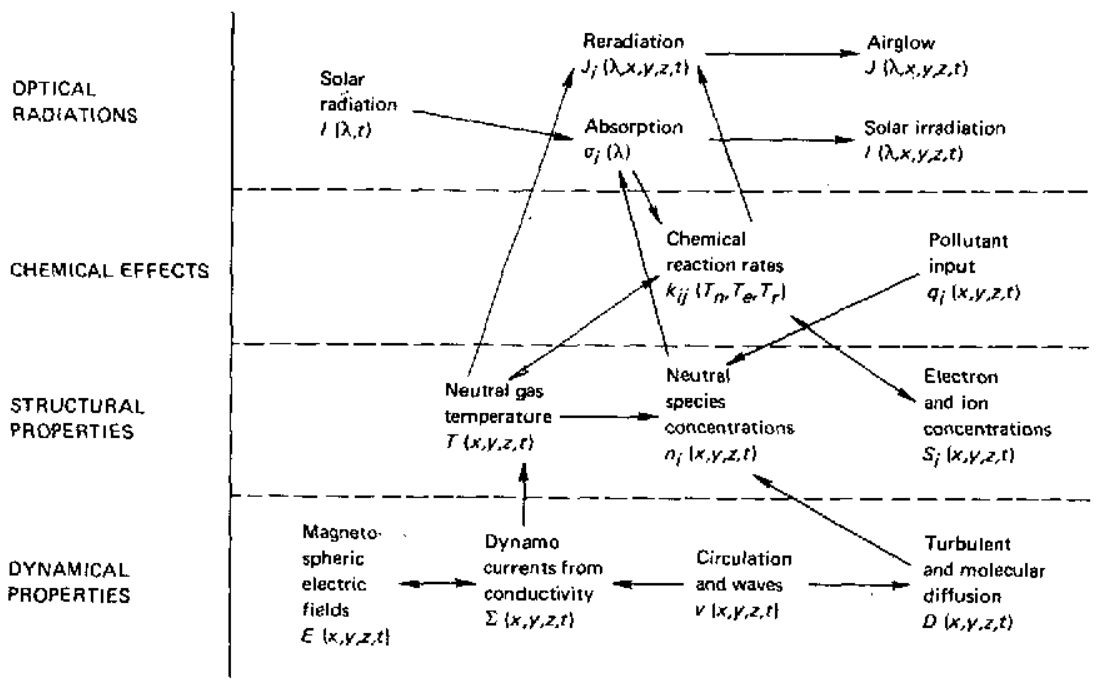

FIGURE 2 Interactive scheme of the atmosphere below $120 \mathrm{~km}$ (excludes particle precipitation, cosmic radiation, and meteor influx). 


\section{Thermal Structure and Dynamics}

The global thermal structure of the stratosphere and mesosphere is important because of its role in chemical, dynamical, and radiative processes. For example, many of the reaction rates involved in the ozone chemistry are strongly temperature-dependent, and the pressure systems arising from heating form the driving force for horizontal motions in the atmosphere.

There are several energy inputs into the atmospheric region, $30-120 \mathrm{~km}$, that are significant from the thermal viewpoint, including solar ultraviolet and $\mathrm{x}$ rays, auroral particles, Joule heating from electric currents, and the breaking of internal gravity waves. Energy losses are by infrared radiation. In addition, there is a massive transfer of energy within this atmospheric region by transport by large- and small-scale motions. The amount and distribution of ozone are very important in controlling the energy input, and knowledge of ozone concentrations near $30 \mathrm{~km}$ (and perhaps down to $15 \mathrm{~km}$ ) will be of value in making clear the nature of the mixing processes.

The temperature distribution up to $80 \mathrm{~km}$ should be measurable by remote sensing using the edge of the $15-\mu \mathrm{m}$ absorption band of $\mathrm{CO}_{2}$, from 80 to $120 \mathrm{~km}$ by laser probing of the sodium layer and from 100 to $120 \mathrm{~km}$ from observations of the profiles of airglow lines. Temperature profiles in the $80-120 \mathrm{~km}$ region provide considerable information about atmospheric structure. Motions in the stratosphere and mesosphere are important because of their role in transporting long-lived chemical and other trace constituents. The motions significant for transport occur on different space and time scales and may be categorized as follows:

1. Zonal-wind and meridional-circulation systems,

2. Planetary waves,

3. Synoptic scale $(\sim 100-1000 \mathrm{~km})$ and mesoscale motions,

4. Gravity waves,

5. Small-scale turbulent motions.

The following are some of the questions concerning thermal structure and dynamics that can be answered by remote sensing from above in conjunction with data from ground-based and rocket sensors:

1. What are the propagation characteristics of the diurnal and semidiurnal tides in the transition region between the mesosphere 
and thermosphere where the wind field changes from a near geostrophic pattern (controlled by Coriolis force) to an ageostrophic pattern (influenced by molecular and eddy viscosity, ion drag, and electric fields)? Can these tides be seen in measurements of neutral temperatures and concentrations by topside remote sensing?

2. What are the turbulent transport properties of the mesosphere, and how do they affect the wind field, the composition, and the energy budget of the atmosphere?

3. How significant in the mesosphere and lower thermosphere are gravity waves excited in the lower atmosphere: what is their energy deposition, and what disturbances of the ionosphere do they produce? Are these gravity waves related to thunderstorm activity and earthquakes? Can they be detected by remote sensing from above?

4. How is the large-scale circulation related to topography? What is the global effect of this circulation on chemically active species?

5. What are the mesopheric chemical processes that determine the distribution of $\mathrm{O}$ and $\mathrm{H}$ ? What is the global pattern of their distribution?

6. How important is the transport of chemical energy associated with the global wind circulation to the energy budget of the mesosphere? What correlations can be seen between the winter anomaly and horizontal thermal structure?

7. What is the cause of the semiannual variations in the lower thermosphere? How is magnetic storm activity associated with Joule heating and particle precipitation, or how is nonlinear coupling from the annual circulation involved? What is the relationship between the mesospheric and thermospheric variations, and what are the coupling mechanisms?

8. What is the relative importance of transport induced by wind circulation above the turbopause and variations in the eddy diffusion at lower altitudes for producing the observed winter bulges in the $\mathrm{O}$ and $\mathrm{He}$ and other atmospheric anomalies? Are similar effects observed in $\mathrm{H}$, and what are the implications for the structure of the geocorona?

\section{Neutral-Atmosphere Chemistry below $120 \mathrm{~km}$}

Compared with studies of other regions of the atmosphere, research on the chemistry of the stratosphere and mesosphere has been limited and fragmentary. In the past two years, interest in these 
regions of the atmosphere has increased markedly because of the possible deleterious effects of the effluents from supersonic transports on the ozone column density. Before detailed predictions of the effects of these added gases and particulates can be made, the natural atmosphere must first be understood. For example, the isotopic abundances of nitrogen in nitrous oxide and other nitrogen oxides are different depending on whether the source is the upper atmosphere or the earth's surface, because of isotopic separation by biological processes in the surface source. The possibility of remote sensing of the abundances and isotopic ratios $\left({ }^{14} \mathrm{~N} /{ }^{15} \mathrm{~N}\right)$ for $\mathrm{N}_{2} \mathrm{O}$ should illuminate the nature of the vertical transport processes.

It appears that the ozone distribution is controlled chemically by the reaction

$$
\mathrm{O}+\mathrm{O}_{2}+M \rightarrow \mathrm{O}_{3}+M
$$

followed by the catalytic cycle

$$
\begin{gathered}
\mathrm{NO}+\mathrm{O}_{3} \rightarrow \mathrm{NO}_{2}+\mathrm{O}_{2}, \\
\mathrm{NO}_{2}+(\mathrm{O} \text { or } h v) \rightarrow \mathrm{NO}+\left(\mathrm{O}_{2} \text { or } \mathrm{O}\right) .
\end{gathered}
$$

A complex chemistry involving water and related radicals or dissociation products such as $\mathrm{OH}, \mathrm{HO}_{2}, \mathrm{H}$, and $\mathrm{H}_{2}$ also plays a significant role. An excited state of oxygen, $\mathrm{O}\left({ }^{1} D\right)$, present in 1 part in $10^{21}$ at the surface of the earth may be of controlling importance. However, it is also quite clear that vertical and horizontal transport of ozone and the atmospheric constituents in its life cycle are important in controlling the distribution. In turn, changes in ozone abundance by as little as 2 or 3 percent may produce biologically significant changes in the ultraviolet irradiation on earth. It will be necessary to understand the circulation system as well as the chemistry before we understand this vital part of the atmosphere.

There is little prospect that a net of rocket, balloon, aircraft, and ground-based sounding stations sufficiently extensive to define the circulation will be set up by 1980 . On the other hand, there are remote-sensing techniques available for use in orbit that offer the possibility of obtaining three-dimensional data on the distribution of a significant number of the important species that play a role in the mesosphere and stratosphere. Specifically these are $\mathrm{O}, \mathrm{O}_{3}, \mathrm{NO}, \mathrm{OH}$, $\mathrm{CO}_{2}, \mathrm{CH}_{4}$, and possibly $\mathrm{H}_{2}$; we discuss these techniques in Section V.A.1. However, optical techniques cannot measure all constituents through the entire height range, and vertical profiles by in situ sensors carried on rockets will be needed at a limited number of locations. 
Thus, the major questions on the chemistry of the stratosphere and the mesosphere that must be answered are the following:

1. What is the chemistry of the natural stratosphere? How can it be studied by remote sensing of the structure, composition, and temperature?

2. What are the relative roles of chemistry, dynamics, and radiative processes in the stratosphere and mesosphere? Can these be revealed by long-lived constituents used as tracers?

3. What are the vertical distributions of the minor atmospheric species, what processes control these distributions, and what role do these species play? How do they vary diurnally, seasonally, and globally?

4. How are the aerosols distributed with height, and how are they formed? What effect do they have on the chemistry of the stratosphere? Can they be explored by topside remote sensing?

\section{E. Ion Chemistry of the D-Region and Lower E-Region}

The circulation, waves, and mixing in the neutral atmosphere between $60-$ and $120-\mathrm{km}$ altitude affect the ionization distribution in three ways:

1. By changing the concentration of minor neutral constituents that produce or modify the ions;

2. By interacting with the earth's magnetic field to move the ionization vertically, sometimes compressing it into thin layers (sporadic E);

3. By changing vertical gradients of ionization by mixing to give random fluctuations in electron density that can scatter radio waves.

These interactions are illustrated in Figure 3. The sensitivity of the ion concentration to motion fields below $120 \mathrm{~km}$ is further illustrated by the lifetimes against recombination of the various species at $10^{3} \mathrm{~cm}^{-3}$ concentration:

hydrated positive ions

$\mathrm{NO}^{+}, \mathrm{O}_{2}+$ ions

hydrated negative ions

meteoric ions
$100 \mathrm{sec}$

$0.5 \mathrm{~h}$

$6 \mathrm{~h}$

1 week 


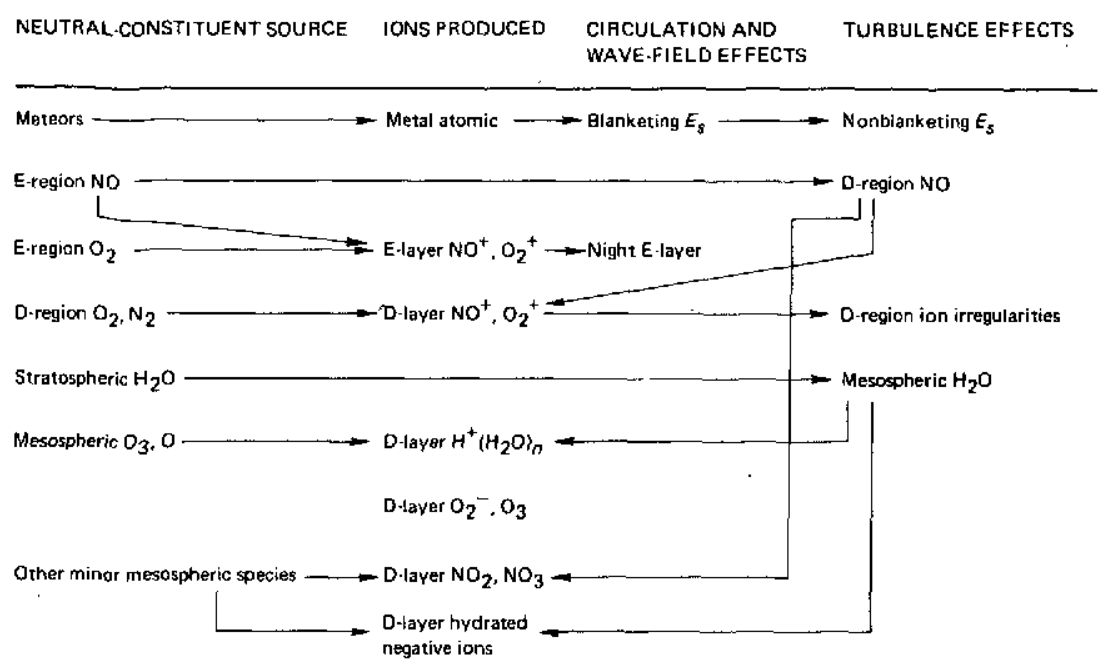

FIGURE 3 Effects of neutral-atmosphere motions on D- and E-region ionization.

As is evident from Figure 3, the ionization distribution is a sensitive indicator of changes in neutral chemistry and transport. Ground-based and rocket measurements have already demonstrated great variabilities in the ionization structure in the D-region. Among the questions that arise are the following:

1. What is the cause of the steep ionization gradient at $80-85 \mathrm{~km}$ altitude and its variability in height? Is the variation associated with temperature or composition changes in the mesosphere?

2. What effects produce the variability of the ion concentration during winter of an order of magnitude from day to day at heights of $70-90 \mathrm{~km}$ ? Are they related to local changes in the thermal or dynamic structure or to gross changes in thermospheric circulation?

3. What produces the changes in the D-region in the aftermath of a magnetic storm? Are long-lived mesospheric constituents observed to move equatorward from the polar regions at this time?

\section{MAGNETOSPHERIC DYNAMICS}

\section{A. Background}

In space physics, prime emphasis is placed on understanding the key physical processes associated with the energy, mass, and momentum 
transfer from the solar wind to the magnetosphere and atmosphere. Available evidence indicates clearly that most of the magnetosphere and the high-latitude ionosphere act as a single closely coupled system. The entire system may be quiescent or disturbed, with the disturbances (called substorms) having characteristic spatial and temporal forms. The fraction of the incident solar-wind energy captured by the magnetosphere may vary more than an order of magnitude, and there exist a variety of paths through which this energy is conducted to the atmosphere, involving direct particle injection, magnetic energy storage, particle acceleration (electrons, protons, and heavier ions), electromagnetic waves, and Joule heating of the ionosphere. Within the closely coupled solar wind. magnetosphere system a number of well-defined boundaries exist between different plasma regimes.

Two additional key elements are motion of the medium and associated electric fields and the wide variety of plasma instabilities that are involved in major magnetospheric processes. For example, it is believed likely that these plasma instabilities give rise to anomalous resistivity that restricts current flow parallel to the magnetic field. This resistivity in turn gives rise to field-aligned electric fields, which contribute to particle acceleration and can enhance or modulate their precipitation into the atmosphere. This precipitation produces auroras, and also ionization, which change the ionospheric conductivity and hence in turn change the general electric field and current distribution. Other instabilities may lead to a self-limitation of the fluxes of stably trapped energetic particles in certain energy ranges, and a very large-scale explosive instability appears to be responsible for the expansion phase of magnetospheric substorms.

Since 1958, a large community of space scientists from many countries has participated in an intensive and successful research effort to study the processes that control the magnetosphere. The result is a morphological description of the magnetospheric field, the particle population embedded in it, and its interface with the solar wind. Space physicists have also identified, and are beginning to understand, many of the physical processes involved.

A number of key steps are required to further the understanding of the dynamics of the magnetosphere. In order to distinguish spatial and temporal variations and to study detailed configuration of boundaries, pairs of satellites are required. A coordinated program of measurements in widely separated regions is required to understand 
the time sequence of events and medium changes in different regions associated with substorm disturbances. This is the main objective of the International Magnetospheric Study (IMS), a coordinated program to be conducted during 1976-1978.

If successful, the IMS program should answer many questions concerning the timing of dynamical changes during substorms and identify the spatial locations of these changes. Progress should also be made on many other questions concerning the solar-wind input to the magnetosphere, the nature of magnetospheric boundaries, and the location of particle acceleration regions. The energy budget in the magnetosphere and ionosphere and the phenomena that control magnetosphere-ionosphere coupling would be the main target of research with the proposed, but not yet approved, Electrodynamic Explorer missions, to be carried out during the period between the IMS and the time at which high-inclination Shuttle orbits become available.

These programs will provide important information on relatively small regions in space and time of processes occurring over very large regions of space, but it is not to be expected that these samples can be fitted in all cases by unambiguous interpretations. Many basic questions will necessarily remain unanswered, many new questions will emerge, and many theories will remain untested after the IMS. To some extent this must be expected because a characteristic feature of the magnetosphere-ionosphere system is that several individual physical mechanisms govern its behavior as parts of complex closed chains of cause-and-effect relationships. A characteristic example is shown schematically in Figure 4. The dynamics of these strong feedback systems will be difficult to understand as long as the dynamic response is studied exclusively through observation of natuial perturbations that are highly unpredictable and whose initial conditions are difficult to establish.

For this reason, after the IMS a new stage of research will become important in which the major emphasis is shifted from "passive" information gathering to a program involving controlled geophysical experiments. These experiments will be designed to break in to some of the chains of cause-and-effect relationships by introducing, at appropriate points, man-made perturbations with various welldefined initial conditions and by determining the ensuing responses. For example, in the diagram shown in Figure 4, several forms of break in are possible: 


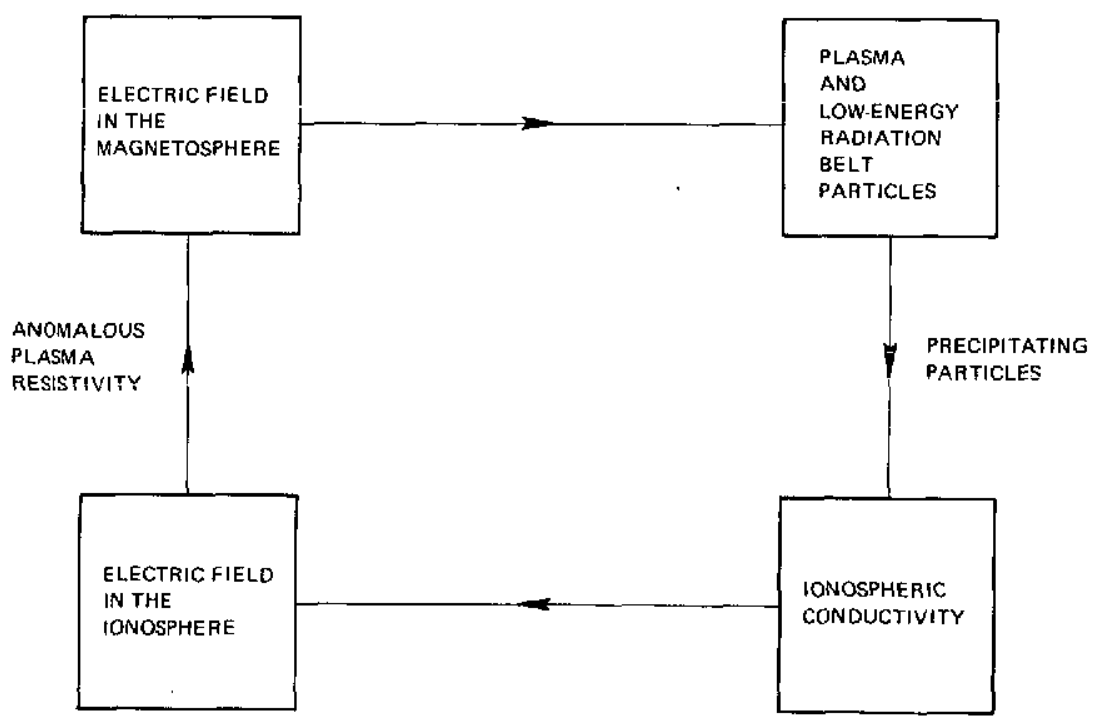

FIGURE 4 Schematic example of a closed chain of cause-and-effect relationships in the magnetosphere.

1. Injecting energetic particles along a field line downward into the lower ionosphere to simulate a beam of precipitating particles of known characteristics;

2. Injecting an intense beam of plasma up a field line or releasing, at large distances, ionized gas from a rocket-launched canister to modify the magnetospheric plasma population under controlled conditions;

3. Modifying the ionospheric conductivity by release of chemicals;

4. Injecting waves of appropriate frequency and intensity to stimulate precipitation via wave-particle interaction;

5. Injecting plasma and/or waves to cause changes in resistivity by enhancing wave-particle scattering.

Systematic man-made perturbations will also serve to stimulate individual mechanisms that are inaccessible to quantitative determination when occurring under natural conditions; in addition they can be used to probe distant regions of the magnetosphere from low-altitude, high-inclination orbits. For example:

1. Release of ions in the solar wind near the magnetopause to 
study the mechanism of particle access into the magnetosphere;

2. Injection of particles upward along a field line to determine parallel electric fields;

3. Injection of particles to probe overall field-line geometry, in particular, the limit between open (tail-like) and closed (dipole-like) field lines.

\section{B. Major Magnetospheric Physics Problems of the 1980's}

Any forecast of the major problems to be faced in a scientific discipline ten years from now obviously has uncertainties, and in the young and rapidly progressing field of magnetospheric science there is much room for change in objectives and priorities. In the following we summarize some of the important questions of magnetospheric physics that will undoubtedly remain unanswered in the 1980's. It is expected that active experiments will play a primary role in any program designed to seek a solution to these problems.

\section{LARGE-SCALE DYNAMICAL PROCESSES IN THE MAGNETOSPHERE}

The large-scale dynamical processes that actually occur in the magnetosphere when such events as substorms, auroras, SAR arcs, and ring current decay events take place are so complex and inherently nonlinear that understanding the basic mechanisms will not be achieved unless a program of controlled experiments is conducted. Some of the main questions to be answered $\backslash$ are the following:

(a) How are the ionosphere and magnetosphere coupled to produce the explosive expansion phase of magnetospheric substorms? Are substorms triggered by perturbations in the solar wind, in the magnetotail, or in the ionosphere? Is substorm occurrence predictable? Injection of beams, waves, and plasma should be used to determine the extent to which substorm perturbations can be produced artificially and to which substorm onsets can be delayed or inhibited by man-made influences.

(b) Are auroral phenomena governed by individual particle effects or collective interactions that develop in the primary auroral beams? Injection of intense particle beams along magnetic-field lines can provide an answer to this question. 
(c) To what extent does the ionospheric conductivity control the magnetospheric convection pattern and the distribution of Joule heating of the ionosphere? Do small-scale changes in ionospheric conductivity affect the location and magnitude of magnetically field-aligned currents? To answer these questions, active experiments, involving artificial modification of the ionospheric conductivity, should be used to determine the role of ionospheric conductivity in the coupling between the ionosphere and magnetosphere.

2. WAVE-PARTICLE INTERACTIONS, PARTICLE ACCELERATION, AND SCATTERING

Although resonant interactions between energetic trapped particles and various wave modes are thought to determine the structure and stability of the radiation belts, few of the important proposed wave-particle interactive processes will have been experimentally confirmed and studied in detail when the Shuttle becomes available. Related problems concern the mechanisms for generation of natural magnetospheric wave modes such as chorus, high-frequency electrostatic emissions, vlf and elf hiss, and triggered emissions. The following are some of the outstanding questions:

(a) How efficient are electrostatic-wave modes as sources for particle energization and pitch-angle scattering? What are the effective coefficients of resistivity, viscosity, and heat conductivity associated with wave-particle interactions? Electron and ion beams injected into the ionosphere and magnetosphere can be used to provide detailed quantitative information on the effectiveness of electrostatic waves for energizing and scattering particles.

(b) To what extent can limiting of stably trapped particle fluxes and observed particle precipitation be ascribed to whistler-mode wave turbulence? To what extent can artificially produced increases of cool plasma density be used to stabilize or destabilize the energetic particle population? Injection of cold ions into the magnetosphere can be used to modify the stability of the whistler mode, thereby modifying the trapped-particle flux and observed precipitation rate.

(c) To what extent is charged-particle precipitation dependent on triggering of instabilities by electromagnetic waves from lightning as opposed to internally generated wave turbulence? Large-amplitude, low-frequency waves can be injected into the magnetosphere by spacecraftborne transmitters to stimulate charged-particle precipitation via resonant wave-particle interactions. 


\section{MAGNETOSPHERIC CONFIGURATION}

Although the gross configuration of the quiet-time magnetosphere will probably be established by the end of the IMS, many detailed questions are expected to remain. Local measurements can never give unambiguous information on large-scale effects. In order to advance the understanding of the overall magnetosphere configuration, it will be necessary in the 1980's to rely on remote-sounding techniques that use charged-particle beams and waves to probe the magnetosphere and tracing experiments that employ charged-particle injections to determine the flow of plasma through the magnetosphere.

Some of the outstanding questions concerning the magnetospheric configuration that can be answered with these active sounding and tracing techniques are as follows:

(a) What are the distributions of parallel electric fields in the magnetosphere? Electron and ion accelerators can be used to study the reflection of charged particles injected along magnetic field lines, thereby providing nonlocal information on parallel electric fields.

(b) Is there a well-defined transition from "open" to "closed" field lines in the nighttime ionosphere? Where is the open- to closed-field boundary in relation to auroral arcs, to the energetic electron trapping limit, and to the inner edge of the plasma sheet? Tracer techniques should be developed to identify and study the boundary between open- and closed-field lines.

(c) From where do the various magnetospheric particle populations come? Specific tracers can be released into the solar wind or deep in the magnetosphere and detected in low earth orbit or on Shuttle-launched subsatellites to provide conclusive answers on particle sources.

\section{PLASMA PHYSICS IN SPACE}

\section{A. Background}

Plasma physics is the basic discipline that is applied in the fields of astrophysics, controlled fusion, and space physics. Many fundamental problems occur in plasma physics that cannot be satisfactorily investigated by ground-based laboratory techniques. Scale lengths encountered in plasmas are frequently larger than the dimensions of available laboratory plasma devices, and wall effects 
and the short duration of discharges often play a dominant role in determining the properties of laboratory plasmas.

The Shuttle sortie missions provide a new and unique tool to investigate fundamental and applied plasma-physics phenomena. All Shuttle orbits are immersed within a natural, unbounded ionized medium with a high vacuum and spatial temporal scale lengths that are much larger than those available in the laboratory. The magnetic pressure is much greater than the hydrostatic pressure, and in extensive orbital regions and in most experimental regimes collisions are negligible. The weightless orbital conditions are extremely important to certain experiments, such as those involving magnetohydrodynanic (MHD) arcs and those confinement devices that are strongly affected by gravity. Since the plasma frequency is of the same order of magnitude as the electron cyclotron frequency, physical effects are brought to the fore that are not usually found in laboratory conditions.

The availability of one or more of these unique space laboratory conditions is of vital importance for some investigations. For instance, the coupling between the long-wavelength radiation field and short-wavelength plasma oscillations is impossible to investigate within the limited confines of any ground-based laboratory. This coupling can introduce coherence in energetic particle streams that may greatly enhance the emitted electromagnetic radiation.

The sortie laboratory missions can also provide the scientific community with opportunities to carry out short-term experiments involving development and testing of new diagnostic devices and investigations of new techniques for plasma propulsion.

\section{B. Outstanding Problems}

\section{GENERAL PLASMA PHYSICS}

The outstanding questions that can be investigated on Shuttle sortie missions include the following:

(a) How do the wave characteristics of each plasma mode, including those with long wavelength, vary with amplitude?

(b) Are there wave-wave and wave-particle interactions that are unexpectedly strong or show peculiar features? Are our basic concepts of weak plasma turbulence theory correct?

(c) Are there new secular effects-both linear and nonlinear-that occur only in large volumes or after a long time? What are the secular effects of resonance excitations? 
(d) Can one take advantage of zero-g conditions to conduct new types of experiments such as those involving convection-free plasma arcs, steady MHD flows, and certain long-term magnetic confinement devices?

(e) How effectively does an antenna radiate electromagnetic waves at low frequencies in a plasma? What is the radiation pattern, and how does the radiation pattern depend on the plasma density and magnetic-field strength?

(f) How does a plasma beam interact with a neutral gas, and what is the role of the gas ionization potential in determining the plasma temperature? What information can be obtained in this way about the interaction between the solar wind and a comet?

\section{WAKE AND SHEATH STUDIES}

The Shuttle will move in the ionospheric plasma with a velocity that is intermediate between the ion and the electron thermal velocities; a complex sheath and wake will form, whose investigation is of interest in itself and to better understand the interaction of the solar wind with planets and satellites. The following are some outstanding questions in this area:

(a) How stable are the wake and sheath regions? How does this stability change when the target is biased? Do Cerenkov cones develop in the wake region?

(b) What is the structure of the shock in front of an obstacle moving at supersonic speed at low-earth-orbit altitudes?

(c) How are phenomena such as antenna impedance and the response of plasma probes affected by the wake and sheath?

(d) Can large-scale terrella experiments be conducted in space using strong deployed magnets? How does the interaction depend on the orientation of the dipole?

\section{DIAGNOSTIC AND PROPULSION DEVICES STUDIES}

Long-standing questions involving the operation of various conventional probes can be attacked with the Shuttle sortie laboratory, making use of precisely known plasma conditions. Moreover, one would like to know the following, for example:

(a) Can one use electron beams to measure longitudinal electric fields over long distances? Can one short-circuit the $\mathbf{v} \times \mathbf{B}$ electric field with an electron beam so that energy can be extracted? 
(b) Can one develop new diagnostic techniques based on plasma resonances suitable fỡ space applications?

(c) Can new propulsion devices, such as the magnetoplasmadynamic arc, be tested and perfected in the unbounded space plasma without the distortions due to finite chamber size and gravity?

\section{INSTRUMENTS AND TECHNIQUES}

\section{A. Atmospheric Science \\ l. CORE INSTRUMENTS}

Most experiments envisaged would involve remote sensing of the atmosphere below either the Spacelab or a free-flyer to determine the spatial distribution-vertical and horizontal-of the constituents of the stratosphere and mesosphere, with sufficient global coverage to enable us to understand the dynamics and chemistry of these regions. The following modes of study are envisaged:

(a) Horizon scanning of selected airglow features by high spectral and spatial resolution photometers and interferometers. A field of view of the order of $3 \mathrm{~min}$ of arc vertical $\times 3 \mathrm{deg}$ of arc horizontal is required to achieve a spatial resolution of $1 \mathrm{~km}$ in the vertical. There is a need for corresponding accuracy in pointing. Such spectral features as the $\mathrm{NO} \gamma$ bands, the $\mathrm{OH}$ electronic transition at $3100 \AA$, the atomic oxygen forbidden transitions at 5577 and 6300 $\AA$, and the Herzberg $\mathrm{O}_{2}$ band system can be studied to give vertical distributions of $\mathrm{NO}, \mathrm{OH}, \mathrm{O}, \mathrm{O}_{2}$, and $\mathrm{O}_{3}$. In the infrared, similar information can be obtained for $\mathrm{CH}_{4}$ and $\mathrm{H}_{2} \mathrm{O}$. Fabry-Perot interferometers will be required as filters to achieve discrimination against Rayleigh scattered sunlight. Input apertures of the order of $50-\mathrm{cm}$ diameter will be required for adequate throughput, and photometers will have to be about $2 \mathrm{~m}$ in length to provide adequate baffling and focal length. A battery of eight such instruments would be required, which would be mounted on a gimbal system about $2 \mathrm{~m}$ long by $2 \mathrm{~m}$ in diameter.

(b) Vertical passive probing by infrared interferometry to determine the vertical distribution of constituents such as $\mathrm{CO}_{2}$ and $\mathrm{O}_{3}$. This requires three instruments: one covering the range $1-5 \mathrm{~mm}$, the other two from 5 to $150 \mathrm{~mm}$; cooled optics and detectors will be required.

(c) Lidar probing of the lower atmosphere. This is a powerful 
technique that should be exploited in the 1980's from spacecraft launched by the Shuttle. Pulses in the ultraviolet (2200-3000 $\AA$ ), generated by tuned lasers, will be returned by Rayleigh scattering at altitudes measured by the time delay. By selecting wavelengths in absorption bands of interesting species and at nearby wavelengths and noting ratios of returned radiation, the abundance of the absorbing species can be determined as a function of the altitude. Alternately, at wavelengths where interesting constituents scatter resonantly the distribution of these constituents may be measured. A third mode of operation involves detection of Raman components of the transmitted pulse scattered by molecular species. It appears that these techniques will permit the measurement of the concentration profiles of the following atmospheric constituents: $\mathrm{O}_{3}, \mathrm{O}_{2}, \mathrm{NO}, \mathrm{OH}$, $\mathrm{H}_{2} \mathrm{O}, \mathrm{NO}_{2}, \mathrm{~N}_{2} \mathrm{O}, \mathrm{CH}_{4}, \mathrm{HNO}_{3}, \mathrm{H}_{2}, \mathrm{CO}_{2}, \mathrm{CO}$, and possibly others. The intensity of the backscattered signal will probably be sufficient to allow the measurement of Doppler shifts produced by horizontal winds. Noctilucent clouds can also be studied. The lidar system would consist of the laser and a receiver $\left(\sim 1 \mathrm{~m}^{2}\right.$ area). Currently tunable lasers of the power envisaged measure $2 \mathrm{~m} \times 1 \mathrm{~m} \times 1 \mathrm{~m}$, but much of this space is occupied by power supplies that could be mounted elsewhere.

(d) Measurement of the absorption of light in selected spectral regions between the Spacelab and a steerable subsatellite. The subsatellite might be a corner reflector located from 1 to $2 \times 10^{3} \mathrm{~km}$ away from the Spacelab in order to return light originating from a laser-telescope system on the Spacelab. Absorption in the ultraviolet would yield the concentration of such species as $\mathrm{O}, \mathrm{H}_{2}$, and $\mathrm{N}$ in the upper atmosphere, while in the infrared the concentration of such species as $\mathrm{O}_{3}, \mathrm{H}_{2} \mathrm{O}, \mathrm{NO}$, and $\mathrm{O}_{2}$ in the lower atmosphere could be measured.

At this time, we foresee a clear need for only a few experiments in the thermosphere above $120 \mathrm{~km}$ after 1980 . Among these would be the release of a chemical such as barium and the observation of the light scattered from it with high spectral resolution to obtain the wind velocity from the Doppler shift of the spectral line and the electric field from the separation of neutral and ionized portions. Natural airglow emissions may also be observed for this purpose. Flexibility of approach will be maintained if provision is made to launch small free-flying subsatellites from the Shuttle for in situ sampling experiments or to allow continued use of other techniques such as sounding rockets. 


\section{MODES}

We foresee using combinations of techniques (a) through (d) to follow the vertical and horizontal motions of atmospheric species as a function of position on the globe and time of day and year. From our knowledge of the behavior of the atmosphere near $100 \mathrm{~km}$, we have reason to believe that time scales as short as one day and as long as three months are involved. Thus, useful information concerning atmospheric chemistry and dynamics could be obtained during seven-day missions from a downward-pointed Spacelab carrying all four experiments. For example, in a high-inclination orbit flown near the solstice and again near the equinox the extreme season-latitudinal variations might be displayed. Universal time and longitudinal effects can also be studied. Alternately, orbits at low inclination would highlight diurnal variations.

Experience suggests that such occasional short samples may miss important long-term effects. Hence we foresee the need to package some of these experiments-selected portions of (a), (b), and (c) for example-into free-flyers. These satellites could be recovered after a suitable period-six months to one year-and reflown after a lapse of one or two years. Both polar and equatorial orbits would be useful. In comparison with the sortie missions, however, these experiments would be comparatively low-data-rate missions and would concentrate on a few key species, $\mathrm{O}_{3}, \mathrm{H}_{2} \mathrm{O}, \mathrm{NO}, \mathrm{OH}$, and $\mathrm{O}$, for example.

\section{B. Space Plasma and Magnetospheric Physics}

\section{CORE INSTRUMENTS AND EQUIPMENT}

The following are considered to be the core instruments and equipment required to perform space-plasma and magnetosphericphysics experiments from the Shuttle during the 1980's:

(a) Electron and Ion Accelerators. Electron and ion accelerators are required to inject energetic beams into the ionosphere and magnetosphere. These accelerators must be capable of providing a wide range of energies (up to $50 \mathrm{keV}$ ) and currents (up to $1 \mathrm{~A}$ ); they must be able to operate in a pulsed mode with pulse durations as short as a few milliseconds.

(b) Plasma Gun. A plasma gun capable of producing intense fluxes of electrically neutral plasma is required to provide high local plasma densities for plasma-physics studies and for injecting high-energy density plasmas into the magnetosphere. A suitable device for generating these plasmas could be a magnetic-plasma dynamic arc of 
the type currently being investigated for plasma-propulsion applications. A typical beam produced by this arc would consist of 10,000 $\mathrm{A}$ of 200-eV argon ions. Operation on hydrogen, all noble gases, and $\mathrm{N}_{2}$ would be possible.

(c) Remote Chemical and Gaseous Injection Devices. Several ejection devices are required to launch canisters containing chemicals and gases from the Shuttle. In most cases, we expect that these devices will be spring ejectors; however, in some instances (e.g., when separations from the sortie lab ranging from hundreds of kilometers to several earth radii are required), rocket launchers will be needed. The canisters to be employed will contain chemicals (such as barium oxide or $\mathrm{SF}_{6}$ ) for release into the magnetosphere, gases for release into the upper atmosphere, and metals to produce meteorlike trails in the lower ionosphere and atmosphere: It may prove preferable in some cases to use coordinated sounding-rocket launches for these releases instead of launching them from the Shuttle.

(d) Transmitters and Antennas. A high-powered radio transmitter and long antennas (up to $1000 \mathrm{ft}$ per element, as on the radio-astronomy Explorer satellites) are required to generate lowfrequency radio waves for probing the ionosphere and magnetosphere. Very large vlf and if voltages, up to $20 \mathrm{kV}$, will be required to produce high-power radiation from this antenna. The major present uncertainty in this area concerns the operation and performance of an antenna immersed in the ionospheric plasma. A separate transmitter and antenna will also be used, covering in frequency range the plasma and the electron cyclotron frequencies. At frequencies well below the local electron plasma and gyrofrequencies, some problems involving tuning, sheath effects, and dipole unbalance arise.

(e) Local Diagnostics. A wide variety of diagnostic instrumentation is requited to provide the measurements of the local environment necessary for properly identifying the basic magnetospheric phenomena of interest. These instruments include magnetometers for magnetic-field measurements, electrostatic charged-particle energy analyzers for energy-spectrum measurements, Langmuir probes for electron-density and temperature measurements, mass spectrometers for local composition measurements, double probes for electric-field measurements, and loop or search-coil antennas for ac magnetic-field measurements.

(f) Long Boom Assemblies. To carry out many experiments, especially those involving low-energy plasmas and small-amplitude waves, it will be required that active perturbing and sensing 
equipment be remotely located from the large Shuttle system. It is anticipated that two retractable $50-\mathrm{m}$ booms, mounted on swivel platforms so that the relative locations of the elements on the ends of the booms can be varied over a wide range, will be needed as a basic element of the sortie laboratory.

(g) Subsatellites. Controllable subsatellites released from the Shuttle are required to perform coordinated observations remote from the sortie lab. With subsatellites, it will be possible to study characteristics of long-wavelength plasma waves and to perform remote studies such as magnetic conjugate-point investigations, which could not be performed otherwise. This subsatellite may be similar in size and capability to the current Atmospheric Explorer spacecraft. The Atmospheric Explorer spacecraft, with its propulsion and spin-up and spin-down capability, is well suited to perform a variety of experiments currently envisaged.

\section{MODE}

Because of the variability and unpredictability of conditions in the magnetosphere, a maximum of flexibility of the experimental setup is required. For instance, for elliptical, high-inclination orbits, the ambient plasma density may change from $10^{6} \mathrm{~cm}^{-3}$ to as low as $10^{2} \mathrm{~cm}^{-3}$ (at high altitudes and high latitudes). The low-altitude range would be best suited for some ionospheric wave-propagation and plasma resonance experiments, while the apogee portions of the orbit would be optimum for collisionless or long-time-scale plasmaconfinement studies. During highly active periods at auroral latitudes, low-energy electron beams could be directed along field lines to detect and measure magnetic-field-aligned electric fields, while during quiet times, high-energy electron pulses could be used to study magnetic-field configuration. Wave injection to stimulate emission of whistler-mode waves will be required for a range of frequencies that generally varies in a known way with latitude but that will also depend to some extent on the conditions at large distances from the Shuttle.

Some specific measurements (not included in all missions) will require the use of small subsatellites in similar orbits for optical and radio transmission (and absorption) studies, and a few flights will require rockets for distant plasma-injection studies.

A well-coordinated, ground-based observational program will be required to complement the active experiments and passive diag- 
TABLE 3 Mission Model for Atmospheric and Space Physics

\begin{tabular}{|c|c|c|c|c|c|c|c|}
\hline & \multicolumn{2}{|c|}{$\begin{array}{l}\text { Free- } \\
\text { Flyers }\end{array}$} & \multicolumn{4}{|c|}{$\begin{array}{l}\text { Seven-Day Sortie } \\
\text { Missions }\end{array}$} & \multirow{2}{*}{$\begin{array}{l}\text { Piggy- } \\
\text { back } \\
\mathrm{G}\end{array}$} \\
\hline & A & $\mathrm{B}$ & $\mathrm{C}$ & $\mathrm{D}$ & $\mathbf{E}$ & $\mathrm{F}$ & \\
\hline Inclination & Low & High & Low & High & Low & High & - \\
\hline Altitude & Low & Low & Low & Low & Low & High & $6.6 R_{E}$ \\
\hline Pointing & Down & Down & Down & Dowı & $\mathrm{Up}$ & Up & - \\
\hline High-power laser and detector system & $\mathrm{X}$ & $\mathrm{X}$ & $\mathrm{X}$ & $\mathrm{x}$ & - & - & - \\
\hline Laser reflector subsatellites & - & - & $X$ & $\mathrm{X}$ & - & - & - \\
\hline Passive optical remote sensing & $\mathrm{X}$ & $\mathrm{X}$ & $\mathrm{X}$ & $\mathrm{x}$ & $\mathrm{X}$ & $\mathrm{x}$ & - \\
\hline Solar instruments & $\mathrm{X}$ & $\mathrm{X}$ & $\mathrm{x}$ & $\mathrm{X}$ & - & - & - \\
\hline Plasma accelerators, electron and ion guns & - & - & $\mathrm{X}$ & $\mathrm{X}$ & $\mathrm{X}$ & $\mathbf{X}$ & - \\
\hline Multipurpose transmitter and antennas & - & ـ & $\mathrm{x}$ & $\mathrm{X}$ & $\mathrm{X}$ & $\mathrm{X}$ & - \\
\hline \multicolumn{8}{|l|}{ Plasma diagnostic instruments } \\
\hline On booms & - & - & $\mathrm{X}$ & $\mathrm{X}$ & $\mathrm{X}$ & $\mathbf{X}$ & \\
\hline On pointed pallet platforms & - & - & $\mathrm{X}$ & $\mathrm{X}$ & $\mathrm{X}$ & $\mathrm{X}$ & \\
\hline Maneuverable subsatellite & - & - & $\mathrm{X}$ & $\mathrm{X}$ & $\mathrm{X}$ & $\mathrm{X}$ & - \\
\hline Local releases and deployment & - & - & $\mathrm{X}$ & $\mathrm{x}$ & - & $\mathrm{X}$ & - \\
\hline Remote releases and deployment & - & - & - & - & $\mathrm{X}$ & - & _- \\
\hline Frequency of flights & $0.5 / \mathrm{yr}$ & 0.5 & $1 / \mathrm{yr}$ & $1 / \mathrm{yr}$ & $0.3 / \mathrm{yr}$ & $2 / y_{I}$ & $0.2 / \mathrm{yr}_{\mathrm{r}}$ \\
\hline
\end{tabular}

nostic measurements conducted from the sortie laboratory. In addition, taking into account the long-proven value of the synchronous orbit for magnetospheric research, small packages should be available in such orbit for coordination with the magnetospheric experiments on the Shuttle.

\section{MISSION MODEL FOR ATMOSPHERIC A N D SPACE PHYSICS}

We summarize here a tentative model in which seven different mission types are listed, based on the use of three different instrumentation groups (Table 3): a single sortie laboratory facility, a single free-flyer, and a single piggyback package. Each sortie laboratory mission carries a maneuverable subsatellite that will generally be deployed and recovered. However, in some cases, it can be anticipated that the subsatellite will remain as a free-flyer for a long-duration investigation.

(A) and (B) Structure, Composition, and Dynamics of the Atmosphere

Objectives: To understand the factors that control the distribution of stratospheric and mesospheric constituents-such as ozone-by determining their diurnal, seasonal, and spatial variations. 
Free-flyers with weight less than $5000 \mathrm{~kg}$, to be flown in nearly circular orbits. The (A) missions are in low-inclination orbits to study aliuinal effects, and the (B) missions are in high-inciination orbits to analyze global and seasonal variations. One pallet is needed to carry other auxiliary optical instruments.

(C) Remote Sensing of the Atmosphere; Local Plasma Physics

Objectives: To measure vertical and horizontal distribution of atmospheric constitutents of the mesosphere and stratosphere and the roles of chemistry and transport and to conduct basic plasmaphysics experiments in the low-altitude, near-equatorial orbits.

The instruments can be mounted on three pallet elements. The energy required is of the order of $500 \mathrm{kWh}$, if the control functions are all carried on board. Since the plasma-physics investigations are to be experiments that must be operated in real time, a man must control the instrumentation based on a real-time evaluation of the data being obtained. He may be in a pressurized module or on the ground, but the latter choice would clearly require a very extensive telemetry and command link to the pallet facility, with continuous orbital coverage.

(D) Remote Sensing of the Atmosphere; Jonosphere-Magnetosphere Coupling

Objectives: To measure vertical and horizontal distribution of atmospheric constitutents of the mesosphere and stratosphere to elucidate the role of chemistry and transport and to carry out controlled study of magnetospheric phenomena.

The instruments can be mounted on three pallet elements with energy requirements of the order of $500 \mathrm{kWh}$ [see comments on man's role in (C) above; for this mission without a pressurized module, continuous telemetry in the polar region is vital].

(E) Solar-Wind Entry and Plasma Injection

Objectives: To investigate motion, stability, and energization of injected plasma clouds and their influence on radiation-belt precipitation.

Rockets would be launched from the Space Shuttle to inject plasma clouds at radial distances 3-15 earth radii with optical observations of the plasma clouds from the Space Shuttle. The total weight is not well defined at present [see comments on man's role in (C) above] . 
(F) Controlled Magnetospheric Experiments; Horizon Scanning of the Atmosphere

Objectives: To study by active techniques the stability of the radiation belts, the phenomena that precipitate the ring current and the trapped particles, and the configuration of the magnetosphere. Laser horizon scanning techniques will be used to study the upper atmosphere.

Three pallet elements to be used, with an energy requirement of $500 \mathrm{kWh}$ [see man's role in (C) above] .

(G) Geosynchronous Diagnostic Package

Objectives: From a remote platform, a $50-\mathrm{kg}$ experimental package is required to study directly the perturbations produced from the Shuttle.

The atmospheric and space physics (A\&SP) sortie mode experiments require manned control, in real time, based on real-time analysis of observational data. This control and analysis can be carried out very efficiently if one to three scientists (of whom the payload specialist may be one) are on board, along with a small pressurized module. All A\&SP sortie missions require about 10,000-12,000 lb of instrumentation placed on three 3-m pallet sections, and inclusion of the pressurized module will not raise the total weight above the allowable Shuttle limits.

It is possible to perform all A\&SP sortie missions in a pallet-only mode, if an adequate real-time tracking and control network is set up to give continuous coverage for all contemplated orbits, including those of high inclination. It should be noted, however, that some of the highest priority A\&SP experiments must be conducted over the polar regions. The proposed Tracking and Data Relay Satellite (TDRS) system will apparently not provide sufficient coverage over the poles. In low-inclination orbits, the pallet-only option appears to be a viable one for many experiments provided the proposed TDRS uplink and downlink systems together with real-time data-handling facilities are established.

\section{R E COM M E N D A T I ON S}

1. Considering the need to conduct experiments in space controlled by man in real time, without interruption during several complete orbits, and taking into account the potential availability of different modes of operation of the sortie lab, we recommend that 
the National Aeronautics and Space Administration and European Space Research Organization conduct a study of the relative advantages in scientific payload weight, cost, available data rate, and coverage of systems using active experiment control based on real-time data evaluation including subsatellite operations, and repair of control instrumentation, of the following modes of operation:

(a) a sortie lab configuration consisting of a pressurized module with three pallet modules and zero to four men in addition to the four-man Shuttle crew, using active control from the pressurized module;

(b) a sortie lab configuration consisting of three or four pallet modules monitored by a payload specialist with active control from the ground.

2. Noting the penalty in payload weight and distribution imposed by the inclusion of the docking module in the sortie lab configurations, we recommend a study by the National Aeronautics and Space Administration and the European Space Research Organization as alternate means of rescue.

3. The scientific observation and experimental program planned in the field of atmospheric and space physics will require certain key instruments or facilities, which will form the nucleus of almost all missions. Consideration of present-day technology and anticipated improvements indicate that suitable instrumentation can be available if a program is started now in certain crucial areas. We recommend that supporting research and technology funds be used to

(a) work on the development of the appropriate lidar technology;

(b) improve general and multiplex detector technology in the optical, infrared, and ultraviolet regions;

(c) develop new in situ sensors for measurement of chemically active minor constituent concentrations in the stratosphere and mesosphere;

(d) develop antenna-transmitter systems for efficient highpower, low-frequency wave generation from within the magnetospheric plasma;

(e) develop, design, and test high-power electron and ion accelerators and plasma beam devices with appropriate energy, density, and beam optics requirements.

It is also important that laboratory studies of reaction rates and theoretical atmospheric modeling studies be continued to allow 
optimal use to be made of the atmospheric data to be gathered by the Shuttle mission.

4. We strongly recommend that an Announcement of Planning Opportunity be issued as soon as possible to select scientists to participate in the detailed scientific definition of the planned programs and the development of the planned instrumentation. 


\section{4 \\ High-Energy Astrophysics}

\section{INT RODUCTION}

High-energy astrophysics involves a wide range of observed phenomena, physical processes, instrumental techniques, and mission requirements. The field includes $x$ rays to the low-energy limit determined by interstellar absorption, gamma rays to the highest energies, and cosmic-ray particles of all varieties and energies. The blossoming of this field and its close relationship with radio and optical astronomy have led to an entirely new view of a universe dominated in large degree by high-energy particles and processes.

Recent discoveries have revolutionized astrophysical thought: the remarkable periodic and pulsed x-ray sources such as Hercules X-1, which can only be explained in terms of compact objects such as neutron stars or black holes revolving in close contact with massive stars; $x$ rays from galaxies and from the intergalactic medium of clusters; a multicomponent diffuse, nearly isotropic background extending over the entire $x$ - and gamma-ray range, which clearly involves the large-scale structure of the universe; gamma rays from point sources and the galactic plane; and, finally, the detection of extremely high- $Z$ cosmic rays, extremely high-energy electrons, the isotopic composition of the lightest elements, and spectral differences of the various components.

In the early period of Shuttle use the opportunity will exist to make detailed measurements of the charge and isotopic composition of cosmic rays, the fine details of the energy spectra to $10^{14} \mathrm{eV}$, and the streaming patterns of low-energy particles outside the sphere of solar influence. We will be able to determine the spectrum and spatial structure of gamma-ray sources in considerable detail, and we believe that detection of nuclear gamma-ray lines from outside the solar system will be possible. The High Energy Astronomical Observatory (HEAO) program should extend the number of $x$-ray sources from 
the presently known 160 to over 1000 and obtain detailed measurements on the spectra, structure, and time variability of these sources.

Focusing $\mathrm{x}$-ray devices on HEAO-B and the larger telescopes of the 1980's will operate as a facility much like ground-based optical telescopes and obtain analogous information arising from entirely different physical processes than those giving rise to optical and radio radiation.

We identify a requirement for an x-ray focusing telescope with an aperture of at least $2 \mathrm{~m}$, during the last half of the 1980's, capable of accommodating a number of instruments at the focus and operated as a national facility. Such a facility will allow us to observe and study high-energy process in the faintest extragalactic objects that will become observable with the Large Space Telescope. Cosmic-ray and gamma-ray research during this period will also require major instruments to determine the spectrum of particles with energies beyond $10^{14} \mathrm{eV}$ and to measure weak fluxes of photons beyond $10^{11} \mathrm{eV}$.

These objectives will require greater resources devoted to this discipline, as well as an increased number of missions, since the field is totally dependent on observations from space. Although we envisage a range of opportunities, only a continuing program of unmanned, long-lived automated spacecraft can provide the continuity of observations required to develop the field and to ensure a succession of new discoveries. The pallet on the Shuttle sortie missions provides opportunities for short observing programs, for development and test of instruments before commitment to longterm flight, and for involvement of many participants in the program, as in the present balloon and rocket efforts. We believe that the program presented here can only be realized if costs are minimized through standardized interfaces, more tolerance of risks, standard spacecraft systems built in quantities, and the operation of large, long-lived instruments as national facilities.

\section{I. S C I E N T I F I C O B J E C T IVES}

\section{A. X-Ray Astronomy}

Discoveries in the past few years have clearly established that $\mathrm{x}$-ray observations are an essential tool in the study of many of the objects of greatest current astrophysical interest such as pulsars, quasars, 
Sey fert galāxies, clusters of galaxiès, and the intergalactic medium. The study of compact $x$-ray emitting objects in binary systems permits investigations of the properties of stars near the end point of stellar evolution and of the physics of matter at extreme pressures, densities, and magnetic fields. In the coming decade, x-ray obseryations will likely be extended to the coronas of main-sequence and giant late-type stars, as well as to peculiar stars such as flare stars. It will also be possible to detect and resolve clusters of galaxies at extreme distances $(Z=3)$ and study their evolution over times comparable with the age of the universe. X-ray emission from clusters of galaxies is likely to originate in the heretofore unobserved intergalactic medium, which may contain a large fraction of the total observable mass of the universe. These studies will profoundly influence our understanding of the dynamics and evolution of the cosmos.

\section{SCIENTIFIC OBJECTIVES}

The scientific objectives of $x$-ray astronomy can be broadly grouped under the following headings:

(a) STELLAR STRUCTURE AND EVOLUTION There is convincing evidence that many of the galactic x-ray sources are binary systems in which one member is a collapsed star, either a neutron star or a black hole. Far from being an oddity, such an x-ray emitting phase appears to be a necessary consequence of present theories in the evolution of stars in close binary systems. (Half of all stars occur in binary systems.) The study of these systems, in which very large amounts of mass are transferred from one member to the other, is essential to the understanding of stellar evolution occurring in these conditions. The presence of neutron stars and black holes in binary systems permits us to obtain a vast amount of information on the physics of highly compressed and nuclear matter. Furthermore, we can for the first time examine dynamical properties in a very intense gravitational field-one in which general relativity effects predominate. Thus we are provided with the equivalent of a general relativity astrophysical laboratory.

The importance of $x$-ray observations of stellar structure is not limited to objects that are primarily x-ray emitters. We can extend to a large range of stars the type of detailed study of stellar atmospheres previously limited to the sun. 
Finally, supernova remnants, for example the Crab nebula, in which most of the electromagnetic energy dissipation occurs via high-energy photons, give us an invaluable astrophysics plasma laboratory for which x-ray observations can be carried out with techniques similar to the ones used in the study of solar plasmas. The study of the generation, containment, and dissipation of the high-energy particles at the pulsar and of the mechanisms of energy transfer to the interstellar medium is of great astrophysical significance.

(b) LARGE-SCALE GALACTIC PHENOMENA X-ray observations provide unique capabilities for studying the interstellar medium. The column density of elements such as oxygen, neon, and sulfur, and possibly the state of ionization of the gas, can be measured directly by observation of the appropriate K-shell absorption edges in the spectra of discrete sources. X-ray observations of the soft background $(\sim 0.25 \mathrm{keV})$, which we believe to be at least in part of galactic origin, can yield information on the structure and distributions of clouds of interstellar material. Such observations can be carried out both of our own galaxy and of galaxies of the local group, such as $M 31$, where we can map the entire galaxy in soft $\mathrm{x}$ rays.

(c). NATURE OF ACTIVE GALAXIES The study of the spatial distribution, spectral characteristics, and time variations of the $x$-ray emissions from the nuclear regions of galaxies could yield the key to the understanding of the fundamental processes that give rise to the enormous production of energy occurring there. In addition; the inverse Compton reaction between cosmic rays and the microwave background, which result in high-energy photons, will allow study of the extended radio regions associated with these objects. Extending the observations to earlier epochs, i.e., greater distances, would allow the study of the evolution of active galaxies.

It is also possible that a new type of extragalactic object has already been discovered, whose detailed properties are as yet unknown, that emits most of its energy in the x-ray region of the electromagnetic spectrum. Approximately 40 of the 160 Uhuru sources appear to fall in this class of "x-ray galaxies." The study of their nature may well turn out to lead to results as startling as the study of quasars. 
(d) RICH CLUSTERS OF GALAXIES-COSMOLOGY The recently discovered extended $\mathrm{x}$-ray emitting regions in rich clusters of galaxies are likely to be a manifestation of a complex intercluster medium. The structure of this region and its relation to the dynamical parameters of the cluster must now be investigated. Observation of very distant members of this class of objects can allow us to study the evolution of the emission regions and of clusters with obvious consequences on cosmological theories. Other $x$-ray observations have direct bearing on cosmological theories, in particular the study of the extragalactic diffused x-ray background. The important question to be resolved is whether the background is due to a large number of individual sources or is truly diffused. In any event the background is a probe into a region of red shift $>3$ and can provide data on the nature and structure of the cosmos on this very large scale.

\section{OBSERVATIONAL OBJECTIVES}

The above scientific objectives can be translated into observational objectives as follows:

(a) HIGH-SENSITIVITY SURVEYS The present limit of $10^{-4} \mathrm{Sco}$ $\mathrm{X}-1$ should be extended to $10^{-8} \mathrm{Sco} \mathrm{X}-1$ with a survey divided into three energy ranges, $\sim 0.1-2 \mathrm{keV}, 2-20 \mathrm{keV}$, and $20-200 \mathrm{keV}$. In at least one of these energy ranges, the surveys must have the following capability: location of point sources to $1 \mathrm{sec}$ of arc, structure of extended sources to $0.1 \mathrm{sec}$ of arc, and broadband spectra $(\lambda / \Delta \lambda \sim 5)$ over the entire range.

(b) HIGH RESOLUTION OF SPECTROSCOPY OF SELECTED SOURCES The sensitivity should be extended to sources of $10^{-4}$ Sco X-1 intensity with $\lambda / \Delta \lambda \sim 10^{3}$.

(c) POLARIMETRY OF SELECTED SOURCES One percent polarization measurements should be made on sources to $\sim 10^{-3}$ Sco X-1 intensity.

(d) STUDY OF TIME STRUCTURE High-time-resolution studies should be made of aperiodic pulsating sources such as Cyg X-1 with $1-\mu \mathrm{sec}$ resolution. In addition, studies of binary periods and changes of the pulsation periods and orbital parameter over 2-4 years should be carried out. Such studies to be conducted on sources of $L_{X}=10^{36}$ $\mathrm{erg} / \mathrm{sec}$ to distances of $30 \mathrm{Mpc}$. 


\section{B. Gamma-Ray Astronomy}

Gamma-ray astronomy provides information that can be obtained in no other way on the high-energy particles and processes occurring in the universe, both currently and in the remote past. Of all parts of the electromagnetic spectrum, only this one measures directly the presence and effects of energetic nuclei and antiparticles, while also preserving the directional and time features of the sources. Nuclear de-excitation gamma rays can uniquely identify places and events in which element synthesis is occurring and give detailed information about what happens in supernova explosions. Furthermore, highenergy electrons, wherever they exist, signal their presence by emitting gamma rays via scattering of the lower-energy radiation present in the same places. This gives knowledge of the electrons independent of assumptions about the magnetic field, on which the radio emission from these electrons depends, and indirectly about the magnetic fields as well-in supernovae, radio galaxies, galactic nuclei or jets, and intergalactic space.

A most exciting recent development has been the discovery from oso.7 measurements of nuclear gamma rays at $0.51,2.2,4.4$, and possibly even $6.12 \mathrm{MeV}$ due to accelerated protons interacting in the solar atmosphere or its surface during intense solar flares. Similar emissions may be expected from objects that exhibit flaring phenomena many orders of magnitude more energetic than that of the sun. These observations herald a major breakthrough in nuclear gamma-ray spectroscopy.

Gamma rays result from quite different mechanisms than those that produce most of the cosmic $\mathrm{x}$ rays, hence they convey different types of information. Moreover, the present universe is extremely transparent to gamma rays, hence they retain the detailed imprint of spectral, directional, and temporal features imposed at their birth, even if they were born deep in regions opaque to visible light or at times far back in the evolutionary history of the universe.

Emission-line spectroscopy will undoubtedly be as significant to gamma-ray astronomy as it has been to astronomical research in the optical and radio regions. In this area, experimental work has been far outpaced by theoretical nuclear astrophysics. Recently, however, gamma-ray line emission during solar flares has been measured, and an indication of monochromatic $0.47-\mathrm{MeV}$ gamma ray from the galactic center has been obtained.

Supernova and blast nucleosynthesis theories have been especially fruitful in posing questions to be answered by nuclear gamma-ray 
spectroscopy. Several researchers have suggested that the exponentially decaying light curve of the type I supernova is related to the radioactive decay of isotopes synthesized in the explosion either by the r-process or by silicon burning. Either process will leave quantities of radioactive materials in the debris, and determination of the presence and constituency of these materials could decide between the mechanisms. In addition to the residual radiation, prompt nuclear emissions from interactions taking place during the explosion could yield information about the supernova processes themselves.

Prompt and secondary nuclear emissions from extragalactic supernova explosions should also be a significant component of the diffuse cosmic gamma-ray background. Since radiation at early epochs will be red shifted, one should see a line profile whose shape is a historical record of the rate of nucleosynthesis in the universe.

Thus, it is no surprise that the gamma-ray sky looks very different from the $\mathrm{x}$-ray sky, which in turn is different from the optical and radio skies. An intensive effort to determine the nature and detailed features of the discrete sources of gamma-ray continua, to measure the spectral structure and understand the origin of the diffuse background and to detect nuclear line radiation from galactic and extragalactic sources, will not only solve or sharply delineate many present astrophysical questions but will set the stage for exciting new discoveries.

It helps in deciphering the origins of the high-energy gamma radiation that the most interesting and likely processes leave characteristic signatures on the spectrum. The interaction of cosmicray particles with gas, producing gammas by $\pi^{0}$ decay, yiclds a spectrum with a broad peak at $68 \mathrm{MeV}$, tailing off gradually to a spectral slope paralleling that of the cosmic rays. Matter-antimatter annihilation also produces gammas via $\pi^{0}$ decay, hence again with a peak at $68 \mathrm{MeV}$; but since annihilation favors nucleons of low velocity, the spectrum falls sharply at energies above a few hundred $\mathrm{MeV}$, instead of following the cosmic-ray spectrum. Scattering of lower-energy photons by high-energy electrons yields a power-law gamma-ray spectrum (if that is the character of the electron spectrum) with a spectral index equal to that of the synchrotron radiation from the same electrons-namely, half the spectral index of the electrons. Other features may be imposed on the radiation by opacity of the universe, produced in the present epoch for gammas above $10^{14} \mathrm{eV}$ by the $2.7^{\circ}$ background and marginally for gammas above $10^{11} \mathrm{eV}$ by the optical background. In the cosmological past, 
the opacities were much higher and the cutoff energies somewhat lower. Red shift due to the universal expansion has moved all features such as these to lower energies in contemporary spectra.

Spatial as well as temporal features are to be sought. For instance, electrons that have escaped from radio galaxies should produce a gamma-ray halo due to scattering of the $2.7^{\circ}$ background during their limited lifetime at high energy. If supernovae are the principal source of cosmic rays in our galaxy, the heavy particle yield per supernova must be large enough that if these particles had remained trapped in the nebula the filaments would have been dragged out more rapidly. Therefore, the fast nuclei must have escaped in the early history and still (because of interstellar fields) inhabit a surrounding region 1 or $2 \mathrm{deg}$ in diameter, where they interact with ambient gas to produce $\pi^{0}$-decay gammas. Massive dark clouds in the galaxy, too, serve as sources of $\pi^{0}$-decay gammas, with which the columnar mass density of the clouds can be mapped. Information already available from SAS-2 shows the presence of spatial structure in the intensity distribution along the galactic plane and also of variations in the spectrum from different directions.

The discovery of unexpectedly high-intensity gamma radiation along the galactic plane in the neighborhood of the galactic center, and of possible discrete sources in this region, is one of the most remarkable outcomes of balloon flights and the few small satellite observations conducted of gamma rays thus far. In order to unfold the structure of the galactic center region, as well as to resolve the other phenomena mentioned above, the energies of high-energy gamma rays must be measured well enough to distinguish differences in the broad spectral features of the different sources; it is vital to measure angles to the smallest fraction of a degree permitted by the fundamental requirement of being able to apply these measurements to extremely small fluxes $\left(<10^{-7}\right.$ photon $\mathrm{cm}^{-2} \mathrm{sec}^{-1}$ in many important cases). Large detector area, at least a few square meters, is therefore a necessity.

In the regime of low-energy gamma radiation, the most distinctive clues are sharp spectral lines, which require fine spectral resolution not only to identify the lines but to discern them in the presence of a strong background continuum. Here, too, adequate sensitivity requires large area.

Possibly the most significant of the gamma-ray discoveries is that of a diffuse high-energy radiation apparently uniformly bright over the entire sky. The existence of this radiation has profound implications for cosmology. It is therefore urgent to measure the 
high-galactic-latitude flux in many directions with enough precision to set fine limits on its anisotropy and to follow its spectrum un to the high energies where opacity in early epochs may have left significant marks. Again, these purposes require very large detector area, fair energy resolution up to high energies, and good angular resolution.

The discrete source studied most extensively is the Crab nebula, from which the pulsed flux has been detected up to more than $10^{9} \mathrm{eV}$. Processes impossible to duplicate in the laboratory, such as gamma-ray absorption via pair production in extremely strong magnetic fields, may be observable in the study of pulsed gammas from neutron stars. The high-energy pulses from the Crab show features on a time scale considerably finer than $10^{-3} \mathrm{sec}$, pointing up the necessity of including the time dimension in gamma-ray detection, to a precision of at ieast $10^{-4} \mathrm{sec}$.

A startling, newly observed phenomenon in need of investigation is the bursts, lasting tens of seconds, of hard $\mathrm{x}$ rays and gamma rays discovered with Project Vela low-energy gamma-ray monitors. What these remarkable events signify is not yet known. They show a need to monitor the whole sky for abrupt changes on as broad a temporal bandwidth and energy bandwidth as possible. Even more clcarly, this recent discovery emphasizes that for gamma rays, as happened before for $\mathrm{x}$ rays and radio waves, the discovery of unexpected phenomena may well outweigh the importance of systematic investigation of known processes. This always happens when one looks at the external universe with new eyes-in a new part of the spectrum or with instruments that have new dimensions of resolution and sensitivity, as do those designed for gamma-ray measurements in the period of Shuttle availability.

\section{Cosmic-Ray Astronomy}

The study of high-energy cosmic-ray particles plays a unique role in modern high-energy astrophysics and has a direct bearing on a variety of basic astrophysical problems. $X$ rays and gamma rays are produced frequently in conjunction with, or by, these high-energy particles. The energy density of cosmic rays in the galaxy, $\sim 1 \mathrm{eV} / \mathrm{cm}^{3}$, is comparable with that of the containing magnetic fields, of starlight, and of the kinetic motion of interstellar matter. The cosmic rays themselves therefore are a major, and perhaps controlling, element of galactic structure.

Cosmic rays provide the only direct material sampies from outside 
the solar system, as well as data on the origin and nature of the most interesting stellar sources, where cosmic rays are believed to originate. These nuclei will have been synthesized in stellar furnaces, then accelerated, ejected, stored, and propagated in the interstellar medium. The physical environments under which nucleosynthesis takes place impart definite signatures to both the charge and isotopic abundances of the manufactured elements. These abundances are altered in a known way by spallation processes in the interstellar medium. After corrections for the propagation effects, the resulting abundances of cosmic-ray sources may be directly compared with predictions from nucleosynthesis theory for different types of astrophysical sources. In addition, the measurement of unique radiojsotopes, which represent "nuclear clocks," can give direct evidence for presently ongoing nucleosynthesis in the galaxy.

Once the particles escape from the vicinity of the sources, they are contained in the microgauss galactic magnetic fields. One sees then a superposition of many sources in which the galactic cosmic rays are largely isotropic upon reaching earth. Beacuse of the containment process, cosmic-ray particles are major elements in the structure and dynamics of the galaxy. The thermal and dynamical state of the interstellar gas, the formation of clouds and stars from the interstellar gas, the structure of the gaseous disk, and the galactic halo are dominated by cosnic rays and can be understood only on the basis of quantitative observations of cosmic-ray charge, energy, and mass spectra. Electrons and positrons have the unique feature that in their passage through the intergalactic medium, they interact with the microwave background radiation, and their observed spectral behavior places constraints on the universality of this radiation. Also these particles are a source of radio waves through synchrotron radiation and of $x$ rays through the inverse Compton process. The interpretation of interstellar processes must take account of all these aspects.

The major observational objectives are

1. To determine accurately, from direct measurements, the energy, mass, and charge spectra of the cosmic-ray nuclei (e.g., H-U and beyond).

The physics of the cosmic accelerators producing the immense energies of cosmic rays is not understood, although a number of ideas involving supernovae, neutron stars, pulsars, and other energetic objects have been proposed. An accurate determination of the energy 
spectra of different nuclei will provide clues to the nature of the acceleration process. Changes in the shape of the energy spectra and the charge and mass distribution at high energies have important astrophysical consequences, often uniquely related to the sources of the nuclei, their storage in the gravitational/magnetic fields of the galaxy, and their extragalactic history. The discovery of even one complex antinucleus such as anticarbon would imply the existence of antimatter stars and element building and would have profound significance regarding the nature of the galaxy and the universe.

2. To determine accurately the energy spectra of cosmic-ray electrons and positrons.

The shape of the high-energy spectrum will provide important clues on the age of the electrons, their source spectrum, the galactic storage mechanism, and their distribution in the galaxy. The galactic electron spectrum below several hundred $\mathrm{MeV}$ is unknown and must be derived from in situ observations in interstellar space. Their flux is importantly related to the production of the diffuse $x$-ray and gamma-ray background in the galactic disk, to the dynamics of the galactic disk-halo configuration, and to the galactic nonthermal radio emission and derived data on interstellar matter and temperature distributions.

3. To measure the energy spectra and elemental and isotopic composition of low-energy nuclei $\left(s 10^{9} \mathrm{eV}\right)$ and electrons in situ in the interstellar medium.

It is not possible to measure the characteristics of the galactic flux of low-energy particles ( $\lesssim$ several hundred $\mathrm{MeV}$ ) near the earth, since the effects of the solar wind prevent their penetration to a heliocentric radius of $1 \mathrm{AU}$. However, in order to reach a complete understanding of the source characteristics and dynamics of galactic cosmic rays, including their identification with unique sources, it is essential to extend the spectral coverage to low energies. At low energies, because of the ionization range requirements, we are sampling very local distributions of galactic cosmic rays (e.g., the range of a $1-\mathrm{MeV}$ proton in the typical galactic magnetic fields is $\sim 200 \mathrm{pc}$ ). A comparison of elemental abundances at low and high energies will be crucial to the separation of features related to the cosmic-ray production and subsequent propagation in the galaxy. The bulk streaming patterns of low-energy nuclei are expected to show large anisotropies, allowing the probing of interstellar space over different scale lengths as functions of energy (e.g., 1-MeV protons with a density gradient of $L \sim 200$ pc and a typical galactic 
diffusion mean free path of $\lambda \sim 20$ pc will show an anisotropy of $\delta=\lambda / L$ of about 10 percent). Possible nearby sources, such as pulsars, may be identified by this technique. The specific details of the low-energy spectra, particularly of heavy nuclei, are profoundly important with regard to the role of cosmic rays in the dynamics of the galaxy, in particular the heating of the interstellar medium by ionization loss.

4. To measure the energy spectra and elemental and isotopic composition of solar energetic particles and solar $\mathrm{x}$ rays and gamma rays.

Solar-particle events provide a microcosm of what is happening on a galactic scale. The charge composition of solar particles is a function of both the acceleration process and the source region. Measurements of isotopes such as ${ }^{2} \mathrm{H},{ }^{3} \mathrm{H}$, and ${ }^{3} \mathrm{He}$ give information on the dynamics of the source region. Coupled with particle acceleration are radio, $\mathrm{x}$-ray, and gamma-ray bursts. The time history of particle intensity provides information on the travel of energetic particles through the magnetic irregularities. Observing these features and the development of the magnetic configuration of the solar-flare region offers probably the most favorable opportunity to understand one of nature's acceleration processes.

\section{I. I N S T R U M E N T S}

\section{A. Achievement of Objectives}

High-energy astrophysics involves the detection of an extreme range of information carriers: electromagnetic radiation from soft $\mathrm{x}$ rays of $100 \mathrm{eV}$ to gamma rays of $100 \mathrm{GeV}$ and particles ranging in character from electrons to transuranic nuclei and in energy from $1 \mathrm{MeV}$ to $10^{8} \mathrm{MeV}$. Precision is needed in determining such different parameters as mass, charge, and velocity of the particles and energy, direction, and time of the photons. Clearly, a wide diversity of instruments is needed. With balloons, rockets, small satellites, or as piggyback riders on OSo's, these diverse instruments could be deployed one by one. With more advanced instruments having greater requirements of mass, volume, pointing, and other supporting subsystems, the costs tend to rise; but in the high-energy field great savings are possible by developing the concept of a multiple-experiment pallet and free-flyer in which the subsystems do not need to be duplicated for each experiment. 
Three modes of deployment will be needed to achieve the objectives of these subdisciplines. One is the free-flying observatory, which may contain only a large focusing $x$-ray telescope, a very-large-area collimated $\mathrm{x}$-ray receiver, or a large-area high-energy gamma-ray telescope; or, alternatively, there may be a big platform containing many different experiments on both particles and radiation (including, for instance, a cryogenic magnetic spectrometer for particles, a cooled high-resolution detector for low-energy gamma-ray ines, and several other particle and photon detectors). A second type of mission of great importance is the kind that uses supplementary propulsion to go out of the solar magnetosphere to measure the undisturbed low-energy galactic particle fluxes, to see the directional asymmetry of the galactic particles, and to apply long-baseline timing to $\mathrm{x}$-ray sources and to the mysterious shortterm gamma-ray bursts to determine the directions. The third mode is the performance of attached experiments on sortie pallets. Many of the proposed experiments in high-energy astrophysics are adaptable to this mode. It will be a great step forward from rocket, balloon, and small satellite experiments in terms of observing time and instrument capability while still accommodating experiments in a developmental state, when moderately rapid redirection and readjustment, in response to the experimental results, are vital. This will be the place for exploratory measurements, where instruments are proved out before their commitment on long-duration free-flying observatories and where many important results will be obtained that will guide and enhance the free-flying programs.

of the above modes, it must be emphasized that by far the most important is the frec-flying, long-life observatory. Some of the subdisciplines of high-energy astrophysics have become highly developed areas of precise measurements and sophisticated technology, in which the most significant objectives require the continuity of observations and long observing times, which are properties of the free-flying mode but not of the brief sortie missions. To carry out the long-range programs of x-ray, gamma-ray, and cosmic-tay astronomy with efficiency, reasonable economy, and maximum scientific return, the most profitable use of the Shuttle will be in establishing these freeflying observation platforms and periodically refurbishing them and supplying them with needed expendables to prolong their lifetimes of automated measurements under control from earth. 


\section{B. X-Ray Instruments for the Shuttle Era}

X-ray astronomy has reached a state of development where its scope and methods closely resemble those of other branches of astronomy. Since $\mathrm{x}$-ray observations can only be carried out in space, it is imperative that permanent orbiting observatories, operated as national facilities, be provided-a requirement that can be satisfied economically only with free-flyers. In particular cases, the capability for recovery and refurbishment will decrease operating costs. Most instrument development and specialized observation can be carried out unmanned and are adaptable to the pallet mode on the Shuttle. The ability to recover and refurbish the large permanent facilities is quite important to allow for continuity of observations at moderate cost.

An important concept to reduce the cost of the free-flyer is the commonality of the support system for all subdisciplines of high-energy astronomy. Thus we propose to use a standard spacecraft support system such as the presently conceived mini-HEAO (High Energy Astronomical Observatory) system. The support system includes the telemetry system, power distribution; and attitudecontrol systems. The experiment support structure should be modular to permit instruments or groups of instruments of various sizes. Standard modules would provide for free-flyers from 3-m diameter $\times 3-\mathrm{m}$ length up to the full Shuttle size of $3-\mathrm{m}$ diameter $x$ $18-\mathrm{m}$ length. Two types of mission are envisaged: those in which the instruments' view direction is along the long axis of the spacecraft and those with view directions along the short axis of the spacecraft. Both require $\sim 1$ min of arc pointing, although $\sim 3 \mathrm{~min}$ of arc may be sufficient for the second type of mission.

A mission of each type should be launched in the early 1980's about 4 years after the mini-HEAO launches. These would be of size compatible with the original HEAO. Follow-on missions in the late 1980's would be full Shuttle-sized payloads of each type.

The smallest free-flyer module will continue to be used to carry out more specialized tasks, in the same sense that small-aperture telescopes are still used in optical astronomy.

$\mathrm{X}$-ray astronomy has developed to the point where a large national facility, multiuser, high-resolution telescope is clearly justified by our knowledge of the x-ray sky. Such an instrument is described below. It should receive major financial support commensurate with a 
launch early in the 1980's. The succeeding sections describe more specialized instruments for which prioritics have not been assigned. Priorities will depend strongly on our knowledge at the time of selection. We would presently give high priority to a large-area array for the study of the known variability of $\mathrm{x}$-ray sources, including black hole candidates with microsecond timing resolution. All of these instruments should be multiuser devices, several of them being part of an $x$-ray facility.

The instrument descriptions and scientific justifications follow. Their gross parameters are summarized in Table 4. Instruments for the sortie pallet and for the free-flyer are designated $S$ and $F F$, respectively.

1. High Resolution Imaging Telescopes (0.1-4 keV; free-flyer; focal plane instruments for imaging, spectroscopy, and polarimetry): $\mathrm{S} \mathrm{X}-5, \mathrm{~S} \mathrm{X}-5^{\prime}$, FF X-1, FF X-2.

The fact that practical $x$-ray telescopes can be built that will reflect and focus $x$-ray photons to at least $4 \mathrm{keV}$ allows for observational capability comparable with that available to optical astronomers. In terms of sensitivity and resolution, and in terms of the great diversity of measurements that can be made, there is no other single instrument or set of instruments available in x-ray astronomy that can approach the capability of a large $\mathrm{x}$-ray telescope with a complement of focal-plane instruments. Besides the imaging instruments, the devices that can be used in the focal plane include Bragg crystal spectrometers, grating spectrometers, polarimeters, and solid-state detectors. In addition, the telescope can be used as an element of an objective spectrometer with either a grating or a Bragg crystal. For each of these instruments, with the possible exception of the Bragg crystal spectrometers, the telescope allows for a qualitative improvement over the equivalent instrument built without a telescope.

The facility can be justified purely on the basis of its imaging capability. Because of problems of source confusion, high angular resolution is required to study any sources in the range fainter than $10^{-4} \mathrm{Sco}$ X-1 or sources present in crowded regions such as single galaxies, globular clusters, or clusters of galaxies. The limiting sensitivity should allow reaching sources as faint as $10^{-8}$ Sco X-1 $\left(10^{-15} \mathrm{erg} \mathrm{cm}^{-2} \mathrm{sec}^{-1}\right)$, which is a range in brightness comparable with that achieved in optical astronomy and greater by about an order of magnitude than what is now achieved in radio astronomy. This allows the observation of solarlike coronas to about $50 \mathrm{pc}$, 
galactic x-ray sources to about $30 \mathrm{Mpc}$, and weak extragalactic sources such as NGC 4151 or NGC 5128 to about $10^{3} \mathrm{Mpc}$. Other classes of extragalactic sources such as rich clusters of galaxies, which are much more luminous, are observable to the very edge of the universe. Furthermore, this sensitivity is achieved without any sacrifice in angular resolution, which should be better than $1 \mathrm{sec}$ of arc.

This facility has the power to attack virtually every classical problem in astronomy-evolution of stellar systems, the structure of the galaxy, the nature of galactic nuclei, the origin and distribution of cosmic rays, the structure and evolution of clusters of galaxies, the evolution of active galaxies, or the large-scale structure of the universe.

There are four general sizes of $x$-ray telescope of interest:

(a) Several monitor $\mathrm{x}$-ray telescopes $\left(\mathrm{S} \mathrm{X}-5^{\prime}\right)$ of $30-\mathrm{cm}$ aperture or less to be used in connection with other experiments (for instance, imaging at higher $x$-ray energies) for the study of selected intense objects (for example, the Crab nebula, the Vela supernova remnant). Such devices could be used on the Shuttle or on free-flyers.

(b) A research instrument (S X-5) of the general dimensions of HEAO-B with $60-\mathrm{cm}$ aperture to study selected sources from frequent sortie pallet missions. The angular resolution is of order of 2 sec of arc. Such an instrument, in addition to performing specialized observations for which time at the larger observatories could not be made available, could be used as a test bed for improved focal-plane instruments and for development of new technology.

(c) A 1.2-m aperture $\mathrm{x}$-ray telescope (FF X-1) with better than $0.6 \mathrm{sec}$ of arc resolution and about $9-\mathrm{m}$ focal length. This instrument would have a factor of 10 greater sensitivity than the HEAO-B experiment and would constitute a frontier line research instrument for the early 1980 's.

(d) A 2.2-m aperture $\mathrm{x}$-ray telescope (FF X-2) with $0.1 \mathrm{sec}$ of arc resolution and a factor of 10 greater sensitivity than FF X-1. This facility would make full use of the Shuttle launch capabilities with dimensions of $3-\mathrm{m}$ diameter $\times 17-\mathrm{m}$ length. This is conceived as a national facility of permanent value with very long (10-year) utilization through use of refurbishment and revisit or return.

2. Large-Area Arrays with Concentrators $\left(0.1-4 \mathrm{keV}, 5-50 \mathrm{~m}^{2}\right.$, pallet and free-flyer, resolution $1 \mathrm{~min}$ of arc within a field of view of $1^{\circ}$ ): S X-1, FF X-4. 
TABLE 4 Shuttle Era X-Ray Astronomy Instruments

\begin{tabular}{|c|c|c|c|c|c|c|c|c|c|}
\hline & $\begin{array}{l}\text { Experiment } \\
\text { Number }\end{array}$ & Instrument & Objectives & $\begin{array}{l}\text { Time } \\
\text { Period }\end{array}$ & Size & Pointing & $\begin{array}{l}\text { Power } \\
\text { Weight }\end{array}$ & Constraint: & Recurcty \\
\hline & $\overline{S X-1}$ & $\begin{array}{l}\text { Large area } x \text {-ray } \\
\text { defector with } \\
\text { concentratsr }\end{array}$ & $\begin{array}{l}\text { Survcy for faint sources. } \\
\text { Moderate angular resolu- } \\
\text { lion }\left(-2^{1}\right) \text { for study of } \\
\text { structure and time varia- } \\
\text { lions of sources in } \\
\text { crowded regions. }\end{array}$ & $\begin{array}{l}1980 \mathrm{~s} \\
7 \mathrm{~d}\end{array}$ & $\begin{array}{l}4 \mathrm{~m}^{2} \text { arca } \times 3 \mathrm{~m} \\
\times N \text { (modular) }\end{array}$ & $\begin{array}{l}0.1^{\circ}: \\
1^{\prime} / \mathrm{sec}\end{array}$ & $\begin{array}{l}150 \mathrm{~W} \\
1500 \mathrm{~kg} \\
\text { per } 4 \mathrm{~m}^{2}\end{array}$ & $\begin{array}{l}\text { Gas system: } \\
<28^{\circ}: \\
450 \mathrm{~km}\end{array}$ & Treferred \\
\hline & $\mathrm{S} X \cdot 2$ & $\begin{array}{l}\text { Large area proportional } \\
\text { cuunter array with } \\
\text { optional use of } \\
\text { modulation collimators }\end{array}$ & $\begin{array}{l}\text { Sludy of time vatiability of } \\
x \text {-ray sources la micro- } \\
\text { second time canstants. } \\
\text { Positions and structure of } \\
\text { sources to }-5^{\prime \prime} .120 \mathrm{keV}\end{array}$ & 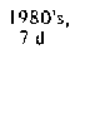 & $\begin{array}{l}5 \mathrm{~m}^{2} 1010 \mathrm{~m}^{2} \text {. Depth } \\
0.5 \mathrm{~m}(1.5 \mathrm{~m} \text { with } \\
\text { modulation sollimatars })\end{array}$ & $\begin{array}{l}0.1^{\circ} ; 1^{\prime} / \mathrm{sec} ; \text {, rotation } \\
\text { aboul view direction for } \\
\text { modulation collimators }\end{array}$ & $\begin{array}{l}1500 \mathrm{~kg}, 5 \mathrm{~m}^{2} \\
22 n 0 \mathrm{~kg}, 5 \mathrm{~m}^{2}+ \\
\text { modulation } \\
\text { collimators; } \\
2500 \mathrm{~kg}, 10 \mathrm{~m}^{2}\end{array}$ & $\begin{array}{l}\text { Cias system: } \\
<28^{\circ} \\
450 \mathrm{~km}\end{array}$ & Preferted \\
\hline & $\$ x-3$ & $\begin{array}{l}\text { Uigh-energy soutces } \\
\text { study }\end{array}$ & $\begin{array}{l}\text { Time variabitity and spectra } \\
\text { of souress } 105 \times 10^{-5} \text { the } \\
\text { Crab intensity. } \\
10-300 \mathrm{keV}\end{array}$ & $7 \mathrm{~d}$ & $1 \mathrm{~m}^{2} \times 1.0 \mathrm{~m}$ & $0.1^{=}: 1$ '/ sec & $100 \mathrm{~W}: 1000 \mathrm{~kg}$ & $\begin{array}{l}<28^{\circ} ; \\
450 \mathrm{~km} ; \\
\text { can fly with } \\
\text { other instru- } \\
\text { ments }\end{array}$ & Preferacd \\
\hline \multirow[t]{6}{*}{ पू } & $5 \times-4$ & $\begin{array}{l}\text { Low-snergy } x \text {-ray } \\
\text { telesiope } \\
(0.6 \mathrm{~m})\end{array}$ & $\begin{array}{l}\text { Study of individual sources } \\
\text { and of interstellar and } \\
\text { intergalactic media in } \\
30-300 \AA \text { range }\end{array}$ & & $0.6-\mathrm{m}$ diam: $3 \mathrm{mu}$ lung & $0.5^{\circ}: 2^{\prime \prime} / \mathrm{sec}$ & $100 \mathrm{~W} ; 1000 \mathrm{~kg}$ & $28^{\circ}$ & Preferred \\
\hline & S X.5 & $\begin{array}{l}\text { High-resolution } \\
\text { imaging telescope } \\
(0.6 \mathrm{~m}) \\
\text { (HEAOH ty pe) }\end{array}$ & $\begin{array}{l}\text { Olsservation of indiyidual } \\
\text { sources with a great } \\
\text { sensitivity, } 10^{-13} \text { erg } \\
\mathrm{cm}^{-1} \sec ^{-1} \text {, and high } \\
\text { angular resnlution }\end{array}$ & $1980^{\circ} \mathrm{s}$ & $\begin{array}{l}\text { 0.6-3n diam; } 5-m \text { focal } \\
\text { length }\end{array}$ & $\begin{array}{l}\text { Accuracy } 3^{\prime}: \text { drift } \\
1^{\prime} / \mathrm{sec}^{-}\end{array}$ & $300 \mathrm{~W}: 2600 \mathrm{~kg}$ & $\begin{array}{l}\because 28^{\wedge} \\
\approx 450 \mathrm{~km}\end{array}$ & Preferred \\
\hline & $5 \times-5^{\prime}$ & $\begin{array}{l}\text { High-resolution } \\
\text { supporting telestope } \\
(0.3 \text { m)(a TM type) }\end{array}$ & $\begin{array}{l}\text { Correlated abservation of } \\
\text { sources with other X-ray } \\
\text { instruments: e.g., } 5 \times-6 \\
\text { for the study of super- } \\
\text { nuvd remnant }\end{array}$ & $\begin{array}{l}\text { larly } \\
1980 \text { s }\end{array}$ & $\begin{array}{l}0.3-m \text { diami } 2.5-m \\
\text { focal length }\end{array}$ & $\begin{array}{l}\text { Accuracy } 3^{\prime} \text { : drift } \\
I^{\prime \prime} / \mathrm{sec}^{\circ}\end{array}$ & $\begin{array}{l}150 \mathrm{w} ; 500 \\
\mathrm{~kg}-?\end{array}$ & $\begin{array}{l}\leq 28^{\circ} ; \\
\approx 450 \mathrm{~km}\end{array}$ & $\begin{array}{l}\text { Si'veral should } \\
\text { be built: } \\
\text { could be } \\
\text { ejected or } \\
\text { recovered }\end{array}$ \\
\hline & $5 \times \cdot 6$ & $\begin{array}{l}\text { Hard } \mathrm{x} \text {-ray imaging } \\
\text { defector }\end{array}$ & $\begin{array}{l}\text { Study of structure of } \\
\text { selemed stou rces down } \\
\text { to }-5^{\prime}\end{array}$ & $7 d$ & $1 \mathrm{~m}^{2} \times 4 \mathrm{~m}$ & $1^{\circ}: 3^{\circ} / \sec$ & $100 \mathrm{~W}: 1000 \mathrm{~kg}$ & $\begin{array}{l}<28^{\circ} \\
450 \mathrm{~km}\end{array}$ & Preferted \\
\hline & $s \times \cdot 7$ & $\begin{array}{l}\text { Bragg crystal } \\
\text { spectrume ters } \\
\text { and polarimeters } \\
0.4-12 \mathrm{keV}\end{array}$ & $\begin{array}{l}\text { Spectroscopy to } \lambda / \Delta x \\
\approx 5000 . \text { Politrimetry to } \\
=2 \% \text { for }-0.1 \text { Crab }\end{array}$ & 70 & $\begin{array}{l}3 \mathrm{~m}^{2} \times 3 \mathrm{~m} \\
\text { for cach instrument }\end{array}$ & $\begin{array}{l}0.1^{\circ} \\
1^{\prime} / \mathrm{sec}^{2}\end{array}$ & $\begin{array}{c}1000 \mathrm{~kg} \\
\text { (cath) }\end{array}$ & $\begin{array}{l}<28^{\circ} \\
450 \mathrm{~km}\end{array}$ & Pretersed \\
\hline & S X-8 & $\begin{array}{l}\text { Broadband spectrometer } \\
\text { with } 5 i(1, j) \text { detectur }\end{array}$ & $\begin{array}{l}\text { Obsirvation of nuclear } \\
\text { gemmat-ray lines and } \mathrm{K} \text {. } \\
\text { lines of high- } Z \text { elements }\end{array}$ & $7 d$ & $1 . \mathrm{m}$ diam $\times 1.5 \mathrm{~m}$ & $0.1^{\circ}$ & $200 \mathrm{w} ; 1000 \mathrm{~kg}$ & $\begin{array}{l}<28^{\circ}: \\
450 \mathrm{~km}\end{array}$ & Precterred \\
\hline
\end{tabular}




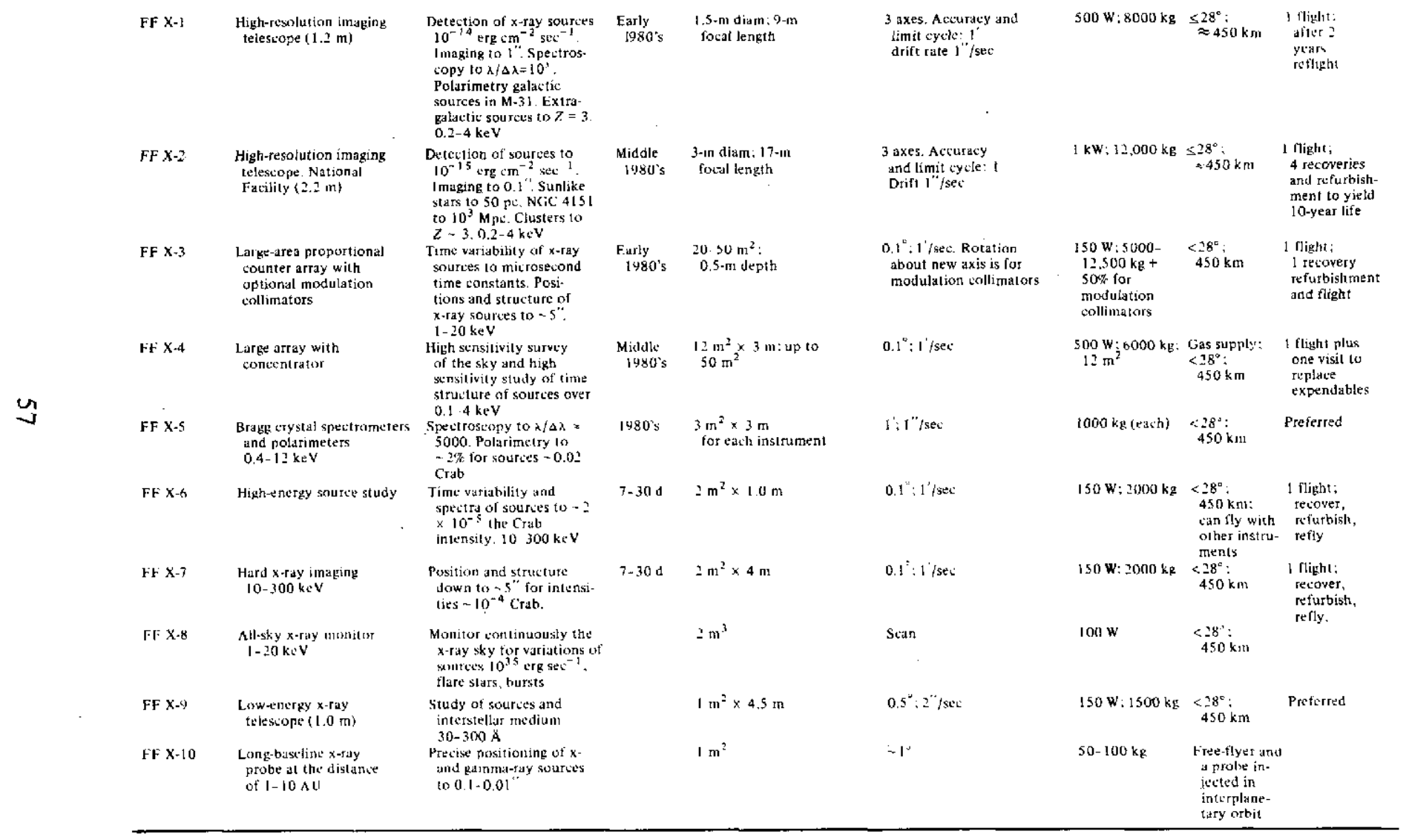


High sensitivity surveys for very faint sources cannot be efficiently conducted with large-area proportional counters with conventional mechanical collimators, because of source confusion. The criterion of 0.03 expected source per resolution element used normally in radio astronomy is already violated in the HEAO-A large-area survey, where extrapolating the results of Uhuru for extragalactic sources one expects 0.3 source per resolution element at the ultimate sensitivity of the survey $\left(\sim 10^{-6}\right.$ Crab). In order to search for even fainter sources, it is necessary to combine large ared with moderate angular resolution.

While focusing $x$-ray telescopes have extremely fine angular resolution (better than $1 \mathrm{sec}$ of arc), they cannot easily achieve large areas. Thus the measurement of the spectrum of the faintest sources, the measurement of time variations of $10^{36} \mathrm{erg} / \mathrm{sec}$ sources in external galaxies, and, finally, the survey of the sky for fainter sources can more conveniently be accommodated by this instrument. Some specific objectives could be as follows:

(a) Ln N-Ln S and spectra of very distant galaxies.

(b) Survey of the sky at $45 \AA$.

(c) X-ray structure of the galaxy.

(d) Properties of x-ray background.

(e) Estimation of absorbing matter in galactic media.

(f) Low-resolution imaging of supernova remnants and similar large-scale features.

(g) Study of x-ray pulses and other variable sources in the galaxy and in $\mathrm{M} 31$.

The large-area arrays are conveniently constructed in modular sections of 1 to $2 \mathrm{sq} \mathrm{m}$ area and about 3-m length.

The achievement of some specific objectives outlined above can be accomplished by Shuttle sortie modes $(S X-1)$ and of others by free-flyers (FF X-4). Items S X-1 and FF X-4 in Table 4 give the gross features of the experiments.

3. Large-Area Proportional Counter Array (1-20 keV; 5-50 $\mathrm{m}^{2}$; pallet and/or free-flyer; collimation variable, e.g., modulation collimators for high angular resolution): S X-2, FF X-3.

A large proportional counter array is the only means presently known to obtain a large effective area for $x$-ray detection in the 3-15 $\mathrm{keV}$ region, where many sources emit most of their energy. The detection of large numbers of photons in short time intervals is essential for the detection and measurement of transient or 
nonperiodic intensity variations. Variations from matter falling into a black hole are expected to have time constants on the order of $10-100 \mu \mathrm{sec}$. The present black hole candidate, Cyg X-1, is known to exhibit aperiodic intensity variations down to an $\sim 50 \mathrm{msec}$ time scale. An effective area of $10 \mathrm{~m}^{2}$ will yield a counting rate of $\sim 10^{5}$ photons/sec for Cyg X-1 or $\sim 1$ photon $/ 10 \mu \mathrm{sec}$. An area of $50 \mathrm{~m}^{2}$ yields 5 photons $/ 10 \mu \mathrm{sec}$. Almost every galactic $\mathrm{x}$-ray source is variable, and transient irregular phenomena are clearly very common. Angular structure of x-ray sources or the background could be studied with modulation collimators mounted upon the detector array. Such studies might be indicated by unusual or energydependent angular structure detected at lower energies by the telescope experiments.

There are a number of different time domains that need to be investigated.

Short Periodic Variability: Periodic components of the emission in the range $1 \mathrm{msec}$ to several seconds are expected based on our present knowledge of pulsars and pulsating x-ray sources.

Short Aperiodic Variability: Certain sources exhibit erratic fluctuations on a short time scale that is important to study. It may be necessary to make measurements in the microsecond range, which is the typical rotation period around collapsed stars.

Eclipse Phenomena: Binary periods are now seen between $5 \mathrm{~h}$ and 7 days. More realistically, one must be prepared to search for periodicities down to the shortest known binary periods, which are about $15 \mathrm{~min}$. The time scale within which the actual eclipse may be is in the minute time range.

Long-Term Variability: Certain objects, such as supernovae, may undergo secular changes in intensity that should be studied in $\mathrm{x}$ rays. Active galaxies also need to be examined for x-ray variability down to the level at which optical variations are now reported. It is quite possible that in these cases the $\mathrm{x}$-ray variability is substantially greater than the optical or radio variability.

Consequently there are requirements both for very-high-timeresolution studies of short (days) duration on specific sources and much longer duration (years) studies of a large number of objects. The first requirement can conveniently be met in a Shuttle sortie mode (S X-2), the other requires a free-flyer (FF X-3).

The construction of such large arrays can usually be made modular with typical modules of 1 to 2 sq $\mathrm{m}$. The items S X-2 and FF X-3 in Table 4 describe the gross features of each experiment mode. 
4. High-Energy X-Ray Source Study (scintillation counters, $10-300 \mathrm{keV}$ range): S X-3, FF X-6.

This will permit detection of sources to an intensity of $5 \times 10^{-5}$ of the Crab nebula and will therefore measure the hard $x$-ray continuum of many x-ray sources. Such data, which contrast the spectrum and time variations in such diverse emitters as the binary $\mathrm{x}$-ray objects, supernova remnants, nonperiodic but varying galactic sources, and extended and compact extragalactic objects, cannot fail to distinguish emission mechanisms and source-region properties. Scintillation counters with active or semipassive shielding configured in modules will be used. An array weighing $\sim 1000 \mathrm{~kg}$ will have an area of $\sim 10^{4} \mathrm{~cm}^{2}$.

This instrument is contemplated for use both in Shuttle sorties and in the free-flyer mode, and the two modes are described under $\mathrm{S} X-3$ and FF X-6.

5. Hard X-Ray Imaging (scintillation counters, modulation collimators, 10-200 keV): S X-6, FF X-7.

Studies of the angular size of extended sources such as the Crab nebula, Perseus cluster, and galactic disk at various energies provide unique information on electron distributions and distinguish between regions of thermal and nonthermal emission in the same object. In the Crab, for example, the higher-energy electrons injected by the central pulsar will diffuse further before being reduced in energy by lifetime limitations. Studies of the Crab require angular resolutions of $10 \mathrm{sec}$ of arc, while nearby clusters of galaxies may have structures in the $0.1^{\circ}$ regime. At present it appears that a modulation collimator on a high-sensitivity scintillation counter device is the best technique for achieving these objectives. The basic sensitivity should be of the order of $10^{-4} \mathrm{Crab}$ in order to obtain a large number of sources. This implies an area of some $10^{4} \mathrm{~cm}^{2}$ in a low-background configuration.

The device can be used in the sortie mode in conjunction with $\mathrm{SX}-5^{\prime}$ to study high-intensity sources and on a free-flyer in conjunction with FF X-1 or FF X-2 for study of fainter sources.

6. Low-Energy Telescope $\left(30-300 \AA\right.$; pallet/free-flyer, $5^{\circ} \times 5^{\circ}$ field of view, focal-plane imaging, spectroscopy, and polarimetry): S X-4, FF X-9.

The opacity of the interstellar medium would appear to severely limit observations between 912 and $20 \AA$. However, the interstellar medium is inhomogeneous, with small dense clouds contained in a 
low-density intercloud medium that can be as low as $N_{H} \sim 0.1$ atom $/ \mathrm{cm}^{3}$. Thus, we can expect to see out of our own galaxy for wavelengths $<100 \AA$ when we look normal to the galactic plane; and even at $304 \AA$, local sources should be observable. The $\mathrm{K}$ and $\mathrm{L}$ absorption edges of the most abundant light elements in the interstellar rnedium are in the xuv. Xuv absorption and scattering can allow us to study the chemical composition and size of the interstellar gains. Attempts can be made to detect the intergalactic medium by means of comparative studies of the detailed shape of the long-wavelength cutoff and the observation of absorption edges in the spectra of the same class of $x$-ray sources from nearby galaxies (such as the Clouds of Magellan) and from more distant galaxies. Discrete xuv emitters that can be studied are chromospheres and coronas of late-type stars, supernova remnants, soft extragalactic sources, early-type stars, and peculiar stars such as flare stars. More hypothetical candidates are defunct pulsars, uv stars, and galactic halos. Focal-plane instruments would include imaging devices, a spectrometer, filters, and a polarimeter.

This instrument is optimized for the study of a presently little explored wavelength region. Thus final definition of a free-flyer instrument should probably await the results of exploratory sounding rocket and/or sortie missions.

7. Bragg Crystal Spectrometers and Polarimeters (1-30 $\AA$, $1.5 \mathrm{~m} \times 2 \mathrm{~m} \times 3 \mathrm{~m}$, effective area $2000-4000 \mathrm{~cm}^{2}$ ): S X-7, FF X-5. A separate crystal spectrometer facility, not in the focal plane of a large $x$-ray telescope, is necessary because the hydrogenic and heliumlike emission lines and the $\mathrm{K}$ absorption edge of iron, as well as of calcium and argon, are at high energies $(E>3 \mathrm{keV})$ not accessible to a focusing telescope; and a specialized crystal spectroscopy instrument will have a much greater collecting area for a given weight and volume. The observation of emission lines or absorption edges will play an extremely important role in the development of our knowledge of discrete galactic x-ray sources, the interstellar medium, and extragalactic sources. The wavelength range from 1 to $25 \&$ will most probably be covered by three crystals. For $\lambda<\sim 6 \AA$, the focusing is accomplished by the configuration of the crystal array itself. At longer wavelengths, the focusing is accomplished by the use of grazing-incidence optics placed behind (or in front of) the crystal array. The extension of the instrument to $100 \AA$ could be carried out with a grazing-incidence grating spectrometer. 
Large arrays of crystals can be used to measure the polarization of the flux from $x$-ray sources. The detection of polarization in the Crab nebula is a clear indication that the synchrotron process is giving rise to the $x$ rays. A 15 percent polarization was detected with a $\sim 0.3 \mathrm{~m}^{2}$ crystal during a rocket flight of $\sim 300$-sec duration.

The detection of faint spectral features or precise determination of polarization requires extremely long observation times. Typically then, the instruments can be tested in Shuttle flights and then should be flown on free-fiyers.

8. Broadband X-Ray Spectrometer (2-50 keV): S X-8.

This instrument will be used to study spectra in an effort to identify specific features such as spectral breaks, nuclear gamma-ray lines, or heavy-element K-line emission. The data necessary for an unambiguous determination of such effects involve both the line profile and the continuum, since, in most instances, physical conditions within the emitting regions will broaden any features. Thus, the need arises for a broadband spectrometer that will give best possible spectral resolution commensurate with available exposure. An array of cooled $\mathrm{Si}(\mathrm{Li})$ detectors will give spectral resolution at least one order of magnitude better than other nondispersive techniques and afford the best means for identification of the broad spectral features predicted for $\mathrm{x}$-ray sources. The above instrument should be developed and tested on Shuttle sorties. Its characteristics are described in $\mathrm{S} \mathrm{X}-8$ in Table 4.

9. Long-Baseline X-Ray Probe (1-10 AU, proportional counters, $1 \mathrm{~m}^{2}$, crystal scintillator $100 \mathrm{~cm}^{2}, 1-\mu \mathrm{sec}$ timing): FF X-10.

The existence of time variations on the time scale of $10^{-2}$ to $10^{-3}$ $\mathrm{sec}$ in several of the galactic x-ray sources makes it possible to determine their position by simultaneous measurement from two detectors placed at planetary distances with a precision greatly superior to any yet achieved in $\mathrm{x}$-ray astronomy (1-0.01 sec of arc). If even faster time variations should occur, the technique could yield positional information to the order of 0.001 to $0.0001 \mathrm{sec}$ of arc. Such a very large increase in angular resolution makes it possible to perform a variety of experiments involving measurement of angular positions as a function of time. Such experiments might lead to precise determination of distance of stellar systems, to the determination of their binary nature, and to refined comparison between structure observed in the $\mathrm{x}$-ray and radio regions. 
The sources of the recently discovered gamma-rays bursts could be pinpointed by this technique also; they may be the direct signal from stellar collapses in other galaxies. The importance of this extension of $x$-ray and gamma-ray techniques cannot be overemphasized. The gross features of the instrument are described under FF X-10 in Table 4.

\section{All-Sky Monitor (1-10 keV): FF X-8.}

The need to monitor continuously the gross intensity variations of the 100 galactic sources presently known has been clearly demonstrated by the Uhuru study of eclipsing binaries of several days' period, by the observation of novalike explosive events, and by the recent indication of new classes of transient phenomena such as the recently observed gamma-ray bursts.

Also, stellar flares of detectable $x$-ray intensity are expected to occur at a frequency of 10 events/year.

In all of these measurements it is necessary to keep the entire sky under observation, with moderate sensitivity, for very long periods of time. The instrumentation could consist of pinhole devices of several different types in conjunction with large imaging detectors. As an example, a set of six simple pinholes each with an imaging proportional counter of about $1000 \mathrm{~cm}^{2}$ could monitor all presently known galactic $x$-ray sources with a time resolution of $\sim 1000 \mathrm{sec}$.

Larger arrays or more efficient imaging schemes would be necessary to monitor sources with resolutions of $0.1 \mathrm{sec}$ as necessary to observe the pulsating characteristics of $x$-ray pulsars and study the variations of their orbital periods.

Obviously such an instrument should be considered for small free-flyers. Its characteristics are described in Table 4 under FF X-8.

\section{Gamma-Ray Experimental Program}

The low flux levels of high-energy quanta, coupled with increasing demand for detailed resolution of source features, drive most gamma-ray experiment designs to include long exposures (a week or more) on individual sources or regions, even with instruments of great area. In order to study many sources or to survey the whole sky, the only possible mode of deployment is then a free-flyer. There are, however, objectives for which an exposure of a few days to a week or two would not only be adequate but in some cases superior. These include specific missions such as a concerted attack on a 
particular source with instruments covering different parts of the spectrum nourited on the same paliet or the attempt to appiy gamma-ray polarimetry to a few of the strongest sources or the exploratory application of a new instrument, where a short period of use could profitably be followed by one of instrument modification before commitment to a free-flyer.

A major subdivision among the gamma-ray experiments occurs between those detecting low- and medium-energy gamma rays, 0.1 to $20 \mathrm{MeV}$, and those detecting high-energy gamma rays, 20 to at least $10^{5} \mathrm{MeV}$. The techniques of detection as well as the observational objectives differ markedly for these two ranges. For instance, only in the low-energy range are sharp spectral lines expected or techniques available for fine spectral resolution; while only in the very high-energy range can presently conceived techniques yield angular resolution of a small fraction of a degree.

The investigations characterized below and in Table 5 include some that are recommended for the sortie mode, especially in the early part of the Shuttle era. Sortic experiment designations begin with the letter $S$ in the table, and free-flyers with FF.

1. High-Energy Gamma-Ray Survey (FF G-1)

This is a full-sky survey at high sensitivity, carried out with a wide-field instrument incorporating moderately good resolution in both direction and energy. This combination of requirements can probably best be met by a pictorial, multiplane wire readout system such as a spark chamber, with a transparent absorbing crystal 6 to 10 radiation-lengths thick for energy determination. With an area of about $8 \mathrm{~m}^{2}$, this instrument will have a sensitivity ten times better than that of the high-energy telescope planned for HEAO-C'. Its angular accuracy will be about $1^{\circ}$ at $100 \mathrm{MeV}$ and $1 / 4^{\circ}$ above 500 $\mathrm{MeV}$, and the energy resolution will be 10-20 percent depending on energy and on thickness of the absorbing crystal. Timing accuracy to at least $10^{-4} \mathrm{sec}$ should be incorporated, since high-energy gammaray sources are known that exhibit pulse structure on that time scale. Aspect information to a precision of $2 \mathrm{~min}$ of arc will be useful.

This instrument will detect point sources down to levels more than 100 times lower than the strongest gamma-ray sources now known, and therefore low enough to include hundreds of examples. It will give spectra accurate enough to determine the main emission mechanism from each source and will locate positions of the stronger sources to $0.1^{\circ}$. For those sources that have angular dimensions on the order of a degree or more, it will map out the source contours. 
For pulsing and eclipsing sources, it will accurately determine pulse profiles and features of the eclipse cycle. The intense radiation from the galactic plane will be mapped out in detail. With the high sensitivity available, exceptionally high-energy gamma rays can be used in this scan of the galactic plane to obtain fine angular resolution, avoid source confusion, and thus distinguish between diffuse origins and an extensive collection of point emitters. The instrument will also obtain a thorough survey and spectral analysis of the radiation from high galactic latitudes and find out whether much of this radiation is in fact isotropic and hence of cosmological origin.

It is urgent that this survey instrument be launched as early as possible because the great wealth of information it will uncover will be of particular value in guiding the rest of the high-energy gamma-ray program.

2. High-Energy Gamma-Ray Pointing Instrument (FF G-2)

In addition to the all-sky survey instrument, a high-energy telescope is needed that will concentrate for longer periods of time on particular sources and areas of the sky of special interest. This is necessary to accumulate the statistical volume of data required to resolve spatial and spectral features of the sources in satisfactory detail and to study temporal variations continuously over periods adequate to decipher complex dynamic behavior.

The field of view of this telescope does not need to be as wide as that of the survey instrument $\left(10^{\circ}\right.$ diameter is enough), but large area is just as essential as in the survey and should be on the order of 5 to $10 \mathrm{~m}^{2}$. This instrument will study single sources or small regions long enough to define some spectra up to $10^{11} \mathrm{eV}$ or more; and significant spectral features in the $10^{10}-10^{11} \mathrm{eV}$ range are predicted for the diffuse radiation and known to occur in at least one source-the Crab pulsar. Therefore, energy resolution to about 20 percent should be provided up. to at least $10^{11} \mathrm{eV}$. Angular resolution should be optimized to the extent possible without loss of sensitivity-at least $0.2^{\circ}$ at energies above $500 \mathrm{MeV}$. Aspect information to $2 \mathrm{~min}$ of arc is desired, although pointing of the vehicle to better than $0.5^{\circ}$ is not necessary. Timing accuracy is needed to at least $10^{-4} \mathrm{sec}$.

This telescope will provide the same types of information as the gamma-ray survey instrument, but in greater detail, extending up to higher energies, and with finer statistical precision, which adds to the accuracy of spectral and angular determinations. Although the 
TABLE 5 Shttle Era Gamma-Ray Astronomy Instruments

\begin{tabular}{|c|c|c|c|c|c|c|c|c|}
\hline $\begin{array}{l}\text { Experiment } \\
\text { Number } \\
\end{array}$ & Instrument & Objectives & $\begin{array}{l}\text { Time } \\
\text { Period } \\
\end{array}$ & Size & Pointing & Weigh1 & Construints & Recorers \\
\hline$F F(;-1$ & $\begin{array}{l}\text { Wide field, high- } \\
\text { energy gamma-ridy } \\
\text { telescope }\end{array}$ & $\begin{array}{l}\text { Whole sky survey: } \\
\text { Positions and specira of } \\
\text { sources down to } 10^{-2} \\
\text { Crab: spectrum and } \\
\text { isotropy of diffuse flux: } \\
\text { structure in galactic plane }\end{array}$ & $\begin{array}{l}\text { As early as } \\
\text { possible in } \\
\text { Shuttle } \\
\text { period }\end{array}$ & $\begin{array}{l}2.5 \mathrm{~m} \times 4 \mathrm{~m} \\
\times 2.5 \mathrm{~m}\end{array}$ & $\begin{array}{l}1: \text { Aspect } \\
\text { measured } \\
10 I \text { min of } \\
\text { are }\end{array}$ & $\begin{array}{l}6000- \\
10,000 \mathrm{~kg}\end{array}$ & $\begin{array}{l}\text { Low-inclination, low- } \\
\text { altitude orbit consistent } \\
\text { with long life }\end{array}$ & $\begin{array}{l}\text { Desirshle hul } \\
\text { not vilat }\end{array}$ \\
\hline FF $(-2$ & $\begin{array}{l}\text { Narrow fie!d, high- } \\
\text { energy gam ma-ray } \\
\text { telescope }\end{array}$ & $\begin{array}{l}\text { Detailed spatial, speclad, } \\
\text { and temporal features of } \\
\text { specific sourcts and areas } \\
\text { of sky }\end{array}$ & $\begin{array}{l}\text { Could be } \\
\text { simultancous } \\
\text { with or some- } \\
\text { what later than } \\
\text { FF } \mathrm{G}_{\mathrm{S}}-\mathrm{l}\end{array}$ & $\begin{array}{l}3.5 \mathrm{~m} \times 3.5 \mathrm{~m} \\
\times 5 \mathrm{~m}\end{array}$ & $\begin{array}{l}0.5: \text { Aspect } \\
\text { meiasured } \\
102 \text { min of } \\
\text { ars }\end{array}$ & $\begin{array}{l}6000- \\
10.000 \mathrm{~kg}\end{array}$ & $\begin{array}{l}\text { Low-inclination, low- } \\
\text { altilude orbit consistent } \\
\text { with long life }\end{array}$ & $\begin{array}{l}\text { Desirable hut } \\
\text { not vital. }\end{array}$ \\
\hline$F F(;-3$ & $\begin{array}{l}\text { Large-area, actively } \\
\text { shielded scintilla* } \\
\text { tiun counters for } \\
\text { low- and medium- } \\
\text { energy gamma rays }\end{array}$ & $\begin{array}{l}\text { High-sensitivity survey of } \\
0.320 \mathrm{MeV} \text { gamma-ray } \\
\text { sources }\end{array}$ & $\begin{array}{l}\text { Moderately } \\
\text { Larly, c.g. } \\
1984\end{array}$ & $\begin{array}{l}1.5-\mathrm{m} \text { diam } \\
\times \quad 1.5 \mathrm{~m} \text { long }\end{array}$ & $0.5^{\circ}$ & $5000 \mathrm{~kg}$ & $\begin{array}{l}\text { Low-radiation and } \\
\text { cosmic-ray background: } \\
\text { no radioactive sources } \\
\text { on board: fow-inclina- } \\
\text { tion, low-altitude orbit }\end{array}$ & $\begin{array}{l}\text { Desirable but } \\
\text { not vital }\end{array}$ \\
\hline$F F \Upsilon_{i}-4$ & $\begin{array}{l}\text { Medium-energy } \\
\text { gatmma-ray monitor }\end{array}$ & $\begin{array}{l}\text { Monitor whole sky for } \\
\text { rapid changes in gamma } \\
\text { intensity : delect direction } \\
\text { spectrum, and time history } \\
\text { of bursts }\end{array}$ & $\begin{array}{l}\text { As early as } \\
\text { possible in } \\
\text { Shuttle eril }\end{array}$ & $\begin{array}{l}1.5 \mathrm{~m} \times 1.5 \mathrm{~m} \\
\times 4 \mathrm{~m}\end{array}$ & $\begin{array}{l}\text { Not needed; } \\
\text { aspect } \\
\text { measured } \\
\text { to } 0.1^{2}\end{array}$ & $2000 \mathrm{~kg}$ & $\begin{array}{l}\text { Not lo be associated with } \\
\text { other instruments need- } \\
\text { ing pointing on same } \\
\text { flyer }\end{array}$ & No \\
\hline $\begin{array}{l}S(i-5 \\
F F=(i-5)\end{array}$ & $\begin{array}{l}\text { High-resolution nuclear } \\
\text { gamma-ray } \\
\text { spectrometer [large, } \\
\text { cooled Ge( Li] de- } \\
\text { tectors with active } \\
\text { shieldingl }\end{array}$ & $\begin{array}{l}\text { High-energy resolution of } \\
\text { nuctear pamma-ray lines } \\
\text { in } 0.05-10 \mathrm{MeV} \\
\text { range }\end{array}$ & $\begin{array}{l}\text { Sortie in early } \\
\text { Shutlle } \\
\text { period: FF } \\
\text { later }\end{array}$ & $\begin{array}{l}\text { I.5-m diam } \\
x \quad 1 \text { in long }\end{array}$ & $0.5^{\circ}$ & $700 \mathrm{~kg}$ & $\begin{array}{l}\text { Low background: no } \\
\text { radiation sources on } \\
\text { board; low-inclination, } \\
\text { low-aflitude orbil }\end{array}$ & $\begin{array}{l}\text { Desirable } \\
\text { hut not vital }\end{array}$ \\
\hline
\end{tabular}




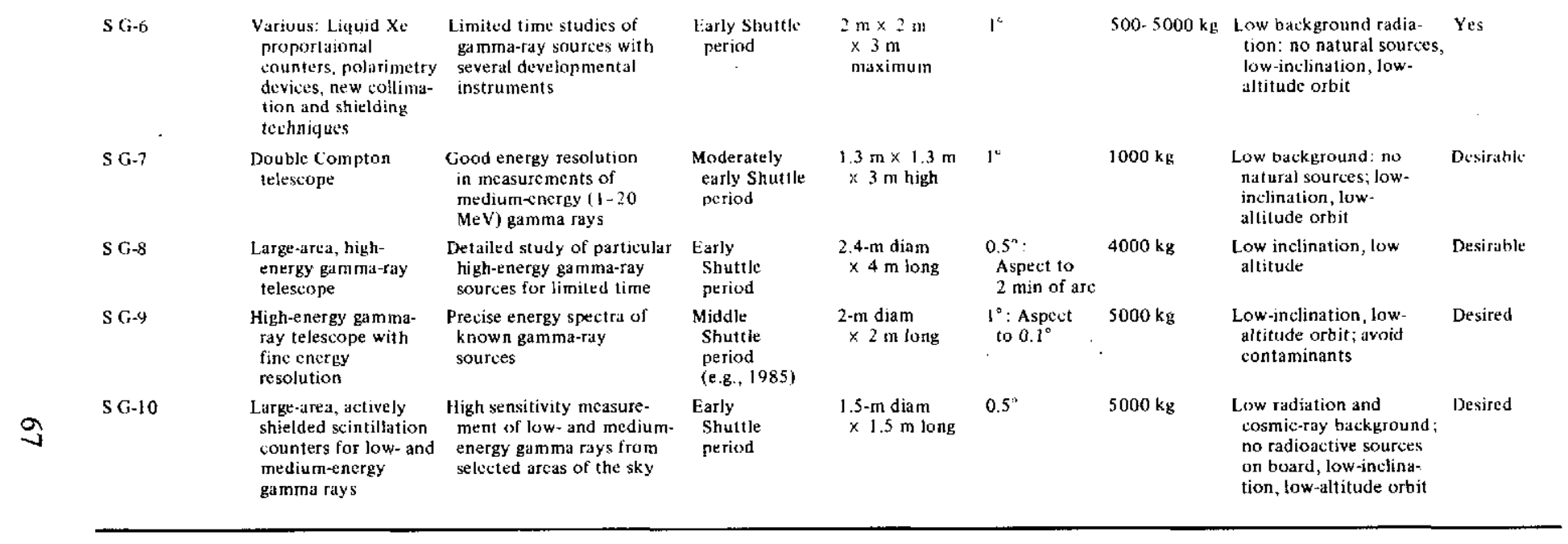


programming of observations with $\mathrm{FF} \mathrm{G-2}$ will profit from the results of FF G-1, it need not wait for the all-sky survey before deployment, since mány exciting targets of ouservation can be defineủ even now, and more will be uncovered by the telescope planned for HEAO-C'.

3. High-Sensitivity, Low- and Medium-Energy Gamma-Ray Survey (FF G-3)

Measuring the spectrum of galactic sources over the $0.3-10 \mathrm{MeV}$ range will determine the relative role of nonthermal electrons and nuclear processes in such objects as pulsating binary x-ray stars, supernova remnants, and the galactic plane. Gamma-ray fluxes at 1 $\mathrm{MeV}$ detect $20-\mathrm{GeV}$ electrons scattering on the $3 \mathrm{~K}$ radiation, which permeates halos and clusters. Detection of perhaps 50 galactic and extragalactic sources requires a cluster of scintillation counters with active anticoincidence shielding, a sensitivity of at least $5 \times 10^{-6}$ photon $\mathrm{cm}^{-2} \mathrm{sec}^{-1} \mathrm{MeV}^{-1}$ at $1 \mathrm{MeV}$, and a total area of $5000 \mathrm{~cm}^{2}$. An aperture of $5^{\circ}$ FWHM represents a compromise between source confusion and sensitivity. These objectives can only be obtained with masssive, large-area detectors and long-term observations.

\section{Medium-Energy Gamma-Ray Monitor (FF G-4)}

The recent unexpected discovery of gamma-ray bursts of less than a minute duration, occurring a small number of times per year, shows the need of maintaining a monitor designed to sense rapid intensity changes in any direction and to record the properties of such events (including direction) that are essential to their interpretation. The types of celestial cataclysms that cause abrupt changes in the gamma radiation are not established, but they include supernovae, the early phase of which ought to be detected in medium-energy gamma rays (the first radiation to emerge) if such an event should happen in the local cluster.

5. Nuclear Gamma-Ray High-Resolution Spectrometer (S G-5, FF G-5)

Determination of the intensities of gamma-ray lines from such processes as radioactivity in supernova remnants, positron annihilation in the galactic disk or in extragalactic interactions, lower-energy cosmic rays passing through dense matter, and nucleosynthesis in violent events in distant galaxies will put nuclear astrophysics on an observational basis. Detection of such lines at a sensitivity of $2 \times 10^{-6}$ photon $\mathrm{cm}^{-2} \mathrm{sec}^{-1}(1 / 10$ that of the HEAO-C' instrument) 
will require a detector of $250 \mathrm{~cm}^{2}$ with active anticoincidence shielding and collimators, and extreme care must be taken to reduce background effects. Present technology indicates that a cooled $\mathrm{Ge}(\mathrm{Li})$ system provides the best energy resolution, although other devices are now under development.

The flight of such an instrument on a sortie pallet early in the Shuttle era will permit a detailed investigation of a few important selected sources and give thorough knowledge of the response of the instrument in the space environment. Later deployment on a free-flyer will permit study of many more sources and improvement of sensitivity by use of long exposures.

\section{Developmental Pallet for Gamma-Ray Experiments (S G-6)}

Many experiments in a developing scientific field, such as low-energy gamma-ray astronomy, require a close and timely interaction between technical developments and scientific discoveries. A system that has basic capabilities for mounting fairly large and massive instruments with modest pointing requirements is needed during the early Shuttle era. This system will serve the same function as rockets for soft $\mathrm{x}$-ray astronomy and balloons for hard $\mathrm{x}$-ray astronomy. Observations that are specific and one-time, such as exploratory polarimetry on strong sources, and observations of new objects found in other wavelengths for phenomena predicted in the gamma-ray regime are examples of possible investigations. Technical developments, such as liquid xenon proportional counters and new shielding or collimation techniques, can have their initial application on such a device.

\section{Double Compton Telescope (S G-7)}

At medium gamma energies (1-20 MeV), both energy and angular resolution are particularly difficult to achieve with conventional single-element detectors. A double Compton telescope is capable of providing good energy resolution in this interval, along with modest angular resolution. The spectrum of selected individual sources will be measured, and also that of the diffuse radiation.

8. Study of Individual High-Energy Gamma-Ray Sources (S G-8)

An instrument similar to FF G-1 or FF G-2, but a slightly smaller area, can be flown profitably on a sortie mission, to perform a detailed study of spatial, spectral, and temporal features of selected areas of the sky. This could be of particular value for (a) the earliest 
application of the instrument, when the results might dictate desirable modifications prior to commitment on a free-flyer; (b) comparatively rapid response to discoveries made in anviner part of the spectrum, which should be complemented by gamma-ray studies of the same sources; (c) investigation of unique objects like the Crab nebula; and (d) a combined attack on a particular source with multispectral instruments mounted on the same pallet, along with coordinated observations from ground observatories.

9. Precise Energy Spectra of Known Gamma-Ray Sources at High Energies (S G-9)

With the aid of a transparent crystal of sufficient thickness to be totally absorbing, gamma-ray spectra from 20 to $10^{5} \mathrm{MeV}$ can be measured with an energy resolution of 3 percent. This capability permits looking for sharp features in the high-energy spectra of selected gamma-ray emitters. Such features, when observed, are highly specific indicators of the physical interactions responsible for the emission and are also capable of identifying previously undiscovered particles and processes.

10. High-Sensitivity Measurement of Low- and Medium-Energy Gamma Rays from Selected Areas of the Sky (S G-10)

An actively collimated scintillator telescope like that of FF G-3 can profitably be deployed in a sortie mission in which the objective is not a complete sky survey but a detailed study of individual sources or areas of the sky. This could be of special value (a) in the earliest application of the instrument, when the results might dictate desirable modifications for the free-flyer; (b) in response to discoveries elsewhere in the spectrum, which should be complemented by gamma-ray observations; (c) for investigation of unique objects such as a young supernova; and (d) as part of a concerted attack on a particular source, using multispectral instruments mounted on the same pallet.

\section{Cosmic Rays}

The anticipated large payload capacities of the Shuttle sortie pallets and of Shuttle-launched free-flyers, with correspondingly large detector systems and observation times, promise major scientific advances from detailed studies of the charge, mass, and energy spectra of cosmic rays. 
The technology to measure charge and energy spectra up to energies of $\sim 10^{15} \mathrm{eV}$ now exists. In particular, the steady evolution of detector resolution means that individual elements from hydrogen through uranium and beyond will be unambiguously resolved. Because of the relatively small fluxes of cosmic rays at higher energies and charges (the intensity scales with energy roughly as $1: 10^{2}: 10^{4} \mathrm{GeV} /$ nucleon $\sim 1: 10^{-5}: 10^{-10}$, and with charge, H:C:Fe: $\left.\mathrm{U} \sim 1: 10^{-2}: 10^{-3}: 10^{-8}\right)$, these high-energy cosmic-ray instruments must be generally massive, of large surface area, and require large observation times. They are dependent on a Shuttle-like capability to carry them above the earth's atmosphere.

In view of the complexity of the instruments, most of the high-energy systems should be test-flown on short sortie missions before being committed to a free-flyer. Such a program allows verification of crucial detector performance parameters. Most of these sortie missions will return scientifically significant results and will meet part of the larger experiment objectives.

\section{Experiments on Low-Altitude Spacecraft}

Table 6 represents a listing of presently identified major highenergy cosmic-ray investigations for the Shuttle era. Estimates are given of what can be achieved on typical 4- 7 day sortie missions and what are the extended capabilities of 0.5-1 year duration free-flyer missions. All listed investigations have no specific pointing requirements beyond a viewing direction away from the earth. The discussion attempts to outline some of the possible experimental techniques and their present state of development and tries to indicate where further improvements are required.

2. High-Energy Spectra and Charge Composition (S C-1, FF C-1)

A class of detector systems has been developed that measures both the energy spectra of cosmic rays over the range of 10 to $\sim 10^{6}$ $\mathrm{GeV}$ and their elemental abundances from hydrogen through iron, with typical resolution of $\leqslant 0.2$ charge unit. These detectors include possible combinations of ionization calorimeters, superconducting magnets, and multithreshold Cerenkov counters. It is possible that transition radiation techniques or other new techniques will permit significant extensions of this range to higher energies within permissible weights.

While typical geometric factors of $\sim 1 \mathrm{~m}^{2}$ sr limit the effective energy range for protons during short sortie missions to $\sim 5 \times 10^{4}$ 
TABLE 6 Shuttle-Associated Cosmic-Ray Investigations-Low-Altitude Spacecraft

\begin{tabular}{|c|c|c|c|c|c|c|c|c|}
\hline $\begin{array}{l}\text { Experiment } \\
\text { Number } \\
\end{array}$ & Investigation & $\begin{array}{l}\text { Measurement Objectives } \\
\text { Sortie Pallet (S) }\end{array}$ & Free-Flyer (FF) & $\begin{array}{l}\text { Weight } \\
(\mathrm{kg})\end{array}$ & Volume & $\begin{array}{l}\text { Power } \\
\text { (W) }\end{array}$ & $\begin{array}{l}\text { Bit } \\
\text { Rate } \\
\text { (kbps) } \\
\end{array}$ & $\begin{array}{l}\text { Orbit } \\
\text { Inclination }\end{array}$ \\
\hline $\begin{array}{l}\text { S C-1 } \\
\text { FF C-1 }\end{array}$ & $\begin{array}{l}\text { High-energy spectra } \\
\text { and charge composition }\end{array}$ & $\begin{array}{l}\mathrm{H}: 10 \leq E \leq 5 \times 10^{4} \mathrm{GeV} / \text { nuclei; } \\
\mathrm{Fe}: 10 \leq E \leq 5 \times 10^{2} \mathrm{GeV} / \text { nuclei }\end{array}$ & $\begin{array}{l}\mathrm{H}: 10 \leqslant E \leqslant 10^{6} \mathrm{GeV} / \text { nucleon; } \\
\mathrm{Fe}: 10 \leqslant E \leqslant 10^{4} \mathrm{GeV} / \text { nucleon }\end{array}$ & $\begin{array}{r}3000- \\
8000\end{array}$ & $(2 m)^{3}$ & 200 & 4 & $\leqslant 28^{\circ}$ \\
\hline $\begin{array}{l}\mathrm{S} \mathrm{C}-2 \\
\text { FF C-2 }\end{array}$ & lsotopic abundances & $\begin{array}{l}E \leqslant S \mathrm{GeV} / \text { nucleon; } \\
1 \leq A \leq 60\end{array}$ & $\begin{array}{l}E \gtrsim 1 \mathrm{GeV} / \text { nucleon; } \\
1 \leq A \leq 250\end{array}$ & $\begin{array}{l}500- \\
6000\end{array}$ & $(1.5 \mathrm{~m})^{3}$ & 200 & 3 & $2\left\{1-55^{\circ}\right.$ \\
\hline $\begin{array}{l}\text { S C-3 } \\
\text { FF C-3 }\end{array}$ & $\begin{array}{l}\text { High. } Z \text { elemental } \\
\text { abundances }\end{array}$ & $\begin{array}{l}E \geq 1 \mathrm{GeV} / \text { nucleon; } \\
20 \lesssim Z \lesssim 50\end{array}$ & $\begin{array}{l}E \gtrsim 1 \mathrm{GeV} / \text { nucleon } \\
20 \leqslant Z \leqslant 92 \\
+ \text { superheavies }\end{array}$ & 5000 & $\begin{array}{l}10 \mathrm{~m}^{3} \\
\times 2 \mathrm{~m}\end{array}$ & 100 & 2 & $213-55^{\circ}$ \\
\hline $\begin{array}{l}\text { S C-4 } \\
\text { FF C.4 }\end{array}$ & $\begin{array}{l}\text { Electron/positson } \\
\text { energy spectra }\end{array}$ & $\begin{array}{l}0.1 \leqslant E \leqslant 10^{2} \mathrm{GeV} \\
\text { charge ratio }\end{array}$ & $\begin{array}{c}10 \leqslant E \leqslant 10^{4} \mathrm{GeV} \\
\text { charge ratio }\end{array}$ & $\begin{array}{r}1000- \\
5000\end{array}$ & $(2 \mathrm{~m})^{3}$ & 200 & 2 & $2 k^{\prime}-55^{\circ}$ \\
\hline
\end{tabular}


$\mathrm{GeV}$, the longer observation times of the free-flyer allow an extension to $\gtrsim 10^{6} \mathrm{GeV}$. This extended coverage is particularly significant since it allows, for the first time, a significant energy overlap with results from extensive air-shower work, thereby establishing a connection with the extremely energetic phenomena $\left(5 \times 10^{14}\right.$ to $5 \times 10^{20} \mathrm{eV}$ ) explored by these ground-based instruments.

\section{Isotopic Abundances ( $\mathrm{S} \mathrm{C}-2, \mathrm{FF} \mathrm{C}-2$ )}

The study of the isotopic abundances of cosmic rays at low and at high energies is a vital but relatively unexplored new area of cosmicray astrophysics. To date only the low-energy isotopes of hydrogen and helium have been measured. However, recent advances in experimental téchniques have produced a number of promising new systems, which make it possible to begin serious study of isotopic composition. At lower energies these include high-precision multiple $-\mathrm{d} E / \mathrm{d} x$ versus range measurements. At moderate energies, the energy range can be extended with Cerenkov counters. At energies of several $\mathrm{GeV} /$ nucleon the use of multiple-threshold Cerenkov counters and the geomagnetic field allows the measurement of mean elemental masses. At these intermediate energies the superconducting magnet also promises to be a very useful experimental tool. The extension of isotope determination to even higher energies represents a formidable experimental task. It involves, most likely, greatly improved Cerenkov counters at low indices of refraction, significant advances in the momentum resolution of magnet spectrometers, and totally new experimental techniques. These developments will benefit particularly from the heavy-ion beams that have recently become available at high-energy accelerators.

\section{High- $Z$ Elemental Abundances (S C-3, FF C-3)}

The study of the abundances of high- $Z$ elements $(Z \gtrsim 26)$ is within the state of the art of present technology. Because of the prevailing extremely low intensities at high energies, these investigations rely on very large-area detectors and long observation times. Proven detection techniques include passive devices, e.g., large plastic sheets, which require recovery for analysis, and large ionization chamber/ Cerenkov counter hodoscopes, which are linked to the observer via telemetry. It is possible to achieve resolution better than a charge unit for individual elements up to and beyond uranium, including the hypothesized "stable islands" of nuclei near charge 115 .

\section{Electron and Positron Energy Spectra (S C-4, FF C-4)}

The intensity of cosmic-ray electrons at a given energy is generally 
on the order of 1 percent of the corresponding nuclear fluxes. Electron detectors therefore have to operate under severe background conditions. Precise spectral information over a large energy range is necessary to understand the interaction of electrons with the cosmic microwave background (inverse Compton effect) and the galactic magnetic field (synchrotron emission). To understand the origin of these particles, it is necessary to measure the sign of their charge. The most straightforward experimental techniques for electron investigations utilize magnetic spectrometers and electromagnetic calorimeters singly or in combination. If the electron spectrum at higher energies continues with a roughly $E^{-2.5}$ power-law shape, present technology allows extension of the measurements up to $\sim 10^{5} \mathrm{GeV}$. At lower energies $(\leqslant 5 \mathrm{GeV})$ proven electron/positron spectrometers exist and await appropriate flight opportunities.

\section{Experiments on Eccentric Orbit and Deep-Space Spacecraft}

In addition to these requirements of high-energy cosmic-ray astrophysics, the Shuttle has an important function as launch platform for both highly eccentric satellites as well as Tug-assisted deep-space missions, which serve the studies of solar energetic particle phenomena and in situ investigations of low-energy interstellar cosmic rays.

Highly developed and sophisticated instruments for measurements of low-energy solar and interstellar particles (with isotope resolution of the order of one tenth amu) exist at present. In the past, these instruments generally were lightweight $(\sim 5 \mathrm{~kg})$, small $\left[<(0.3 \mathrm{~m})^{3}\right]$, with low power consumption $(<10 \mathrm{~W})$. Considerable scientific advances in the study of solar composition and dynamical phenomena can be expected from somewhat larger instruments $(\sim 15-20 \mathrm{~kg})$ flown by the Shuttle. Interstellar in situ observations of low-energy cosmic rays are presently in their infancy. They can be expected to add totally new dimensions to the near-earth studies of high-energy galactic cosmic rays, including the very exciting possibility of identifying spatial features and unique cosmic-ray sources.

\section{E. Shuttle Sortie Mode Requirements}

In order to give input to the design of the Shuttle sortie mode, a number of possible experiment combinations were established and investigated to determine constraints on the sortie mode pallets.

The baseline requirements for these are (a) no man required; (b) 3-m pallet element length, $4.5 \mathrm{~m}$ width; (c) power and telemetry provided by the pallet; (d) 10-15 pallet element flights/year for this discipline. 
As a result of this investigation, the following recommendations on the capability of the pallet and sortie mode are made:

1. Pointing Requirements

Four modes of pointing requirement have been identified:

(a) Experiments are mounted on pallet, Shuttle used for orientation, accuracy to $1^{\circ}$, readout to $0.1^{\circ}$.

(b) Two-axis stabilized platform, full pallet element area. Accuracy $a=0.1^{\circ}$, jitter $b=1^{\prime} / \mathrm{sec}$. This should carry much larger weights than the $2000 \mathrm{~kg}$ presently envisaged, e.g., $5000 \mathrm{~kg}$.

(c) Three-axis stabilized, large-diameter platform. $2000 \mathrm{~kg}$, 2.5-m diameter, 4.5-m length, $a=1^{\prime}, b=1^{\prime \prime} / \mathrm{sec}$.

(d) Three-axis stabilized, small-diameter platform, $1000 \mathrm{~kg}$, $1-\mathrm{m}$ diameter, 2.5-m length, $a=1^{\prime}, b=1^{\prime \prime} / \mathrm{sec}$.

For all oriented platforms, the slewing rate should be $0.5-1^{\circ} / \mathrm{sec}$.

\section{Orbit}

Orbit requirements are for both low-inclination $\left(<30^{\circ}\right)$ and highinclination $\left(30-55^{\circ}\right)$ latitude.

Altitude should be minimum yet be consistent with a 1-year lifetime if free-flyers are ejected.

\section{Thermal Control}

The system should provide sufficient flexibility to provide thermal control in each individual case.

\section{Contamination}

We consider the ATM standards as sufficient for our requirements. There should be no radiation sources on board, and no large changes of background-producing masses or release of large quantities of material should occur. External magnetic fields should be small (roughly a few gauss).

\section{Weight, Power, Telemetry}

Most pallet elements are in the $\$ 6000$-kg payload class. Those that exceed this limit are modularized so that they can be made to fit available weight capabilities. Occasionally weight/pallet element requirements run up to $\sim 10,000 \mathrm{~kg}$. Power requirements are of the order of $\sim 300 \mathrm{~W} /$ pallet element, exclusive of thermal control.

Data rates are usually $10-100 \mathrm{kbits} / \mathrm{sec}$ but may, for short periods up to $1000 \mathrm{sec}$, run up to $10 \mathrm{Mbits} / \mathrm{sec}$. A pallet data storage system of $\sim 10^{10}$ bits and/or periods of telemetry rates exceeding those now planned would be required. 


\section{PR OGRAM IMPLEMENTATION}

The Space Shuttle provides the potential to conduct new and exciting scientific investigations at costs and on a time scale not previously possible from space. The realization of this potential requires the modification of many accepted management practices and the implementation of new practices. The large weight-lifting capability, recoverability, and short turn-around time to reflight are the characteristics that will contribute most to the realization of the high scientific potential at relatively low cost of the Shuttle.

The balloon and sounding-rocket programs have proven highly effective in carrying low-cost, but scientifically valuable, payloads into space. The Working Group recommends extending the balloon and rocket experiment philosophy to the Space Shuttle. Adopting this philosophy requires acceptance of relaxed quality assurance and reliability standards, greater reliability on the performance of the principal investigator (PI), and standardization of most systems interfaces. Large national facilities require a new management/ investigator approach.

\section{A. Single-Investigator Experiments}

Achievement of the goal of performing valuable scientific research at reasonable costs infers placing greater responsibility for development and testing on the PI. That is, the burden of providing a tested, functioning instrument to NASA for integration must be with the Pl. At present, NASA monitors the activities of the PI through a large in-house organization. A more practical and economical approach appears to be to provide the PI with an experiment handbook in which standardized electrical and mechanical interfaces, safety requirements, and launch environment are provided. The burden of meeting these requirements would be on the PI not on the NASA contract monitor. NASA would, however, assure that the intent of the requirements is being met through one or more design reviews early in the development of an instrument. The frequency of review would be determined by the complexity of the instrument. At delivery of the instrument, the integration center would perform functional tests: inspection for adherence to safety requirements and simple, flight-level vibration and thermal tests. If the instrument fails, the flight opportunity would be lost to the PI. When it passes, the instrument would be integrated and launched. The Working Group 
recognizes the necessity for NASA to maintain accountability over public resources. We believe, however, that this can be accomplished with a lower level of monitoring than presently exercised by NASA on the larger projects.

Experiment selection and development and the long lead time from selection to flight are major factors in increasing experiment costs. Under the present syster, an announcement for flight opportunity is issued. Interested investigators submit proposals, which are evaluated by a NASA committee, and a number are selected for flight. Selection is often tentative and is made final only after a 6month or longer definition study. Following formal selection, the PI is funded for major hardware fabrication. The hardware is delivered to the integration center for integration, test, and finally launch. This entire procedure from proposal to launch can often extend over a period of four or more years. During this period, the PI's staff is being funded; the spacecraft contractor has a major design, fabrication, and testing effort in progress; and the NASA management center has a staff monitoring the activities of all participants.

The Working Group proposes a modification of this system into an evolutionary instrument program from Supporting Research and Technology (SR\&T) funding through pallet flight into flight on a free-flying spacecraft. Under the proposed system, a potential PI would be given an agreed upon sustaining level of SR\&T funding. The level would probably be higher than present-day SR\&T levels and would be supplemented on occasion when new construction or modification is required. Within this fixed budget, the PI would develop a new instrument concept and construct the instrument to the relaxed quality assurance standards, much as he does today for a balloon flight. If the instrument is flown successfully (scientifically as well as technically) on the pallet, the same instrument would be upgraded and the interfaces modified as necessary for flight on a free-flyer. It is recognized that an impartial selection committee must be interposed at various stages to advise NASA on specific selection actions. The ability of the Shuttle to check out spacecraft in space, recover malfunctioning spacecraft, refurbish standardized spacecraft, and refly instrumentation enables an investigator to carry through from development to spacecraft flight with a single basic instrument. The PI would, under this system, carry out a complete research program over the period of a decade without the major perturbations of specific flight instrumentation construction or the long and expensive 
lead times from proposal submission to spacecraft flight.

The requirement for a PI to operate on a fixed budget over an extended period of time will also have the beneficial effect of requiring long-term planning of a research program, sharing common components with other Pl's, and integrating design and development into a standardized form that does not require major expenditures for relatively small modifications necessitated by spacecraft interfaces or man rating. Particle physicists using accelerators, for example, often borrow detectors or other major pieces of equipment rather than build an entirely new module for an experiment. The practice of sharing equipment and data output at an accelerator has often been necessitated by funding limitations under which an experimenter must operate. The same philosophy should be applicable to investigations in space.

\section{B. National Facilities}

Large national facilities require major commitments in national resources. This situation requires the establishment of special procedures that assure that the broadest possible segment of the scientific community are able to participate in the benefits of the investment. Where possible, we recommend applying the above procedures to national facilities. In addition we recommend the following implementation procedures for national facilities:

1. A permanent staff should be developed to assume full operational management of the facility when it is ready for flight. This staff may overlap or even coincide with the group that guided the payload through its construction stage or with an enlarged group constituting the Scientific Steering Committee for the program. The staff would have responsibility for the operation of the facility and the execution of its research program.

2. Selection of research programs and of the users should be the responsibility of a research committee of representatives from the high-energy community, NASA, and the permanent staff. This committee would review research proposals to determine their compatibilities and would then judge their scientific worth. When approved, proposals would be given to the permanent staff, who would determine requirements for observations and related support. In order to ensure impartial consideration, the research committee would be composed of representatives from most of the major research centers, and its membership would be changed periodically. 
All users are responsible to NASA for effective use of their assigned observing time, prompt analysis and interpretation of their data, and publication of their findings.

\section{M I S S I O N M O D E L}

The flight program in high-energy astrophysics can utilize the Space Shuttle as a launch and recovery vehicle for unattended automated spacecraft and in the attached sortie mode analogous to the present balloon and sounding-rocket program. The Summer Study group identified approximately 60 high-energy astrophysics groups that are presentiy pursuing active experimental research programs and that are capable of mounting valuable scientific investigations during the period from 1980 to 1991 . The mission model developed here assumes the continuation of the present NASA automated, balloon, and rocket programs as outlined in the NASA mission model through the 1970's. The Space Shuttle is assumed to become available on a limited operational basis in 1980, and fully operational in 1983- 1984.

\section{A. Automated Program}

The Shuttle-launched and -recovered automated spacecraft program is centered on standardized spacecraft of the HEAO class (see Table 7). These spacecraft are visualized as being launched and recovered on a six-month basis: that is, two launch/recovery missions per year. In addition, smaller Explorer-class spacecraft are required on a schedule of about one per year. The exact frequency will depend on the

TABLE 7 High-Energy Astrophysics Mission Model-Automated Spacecraft

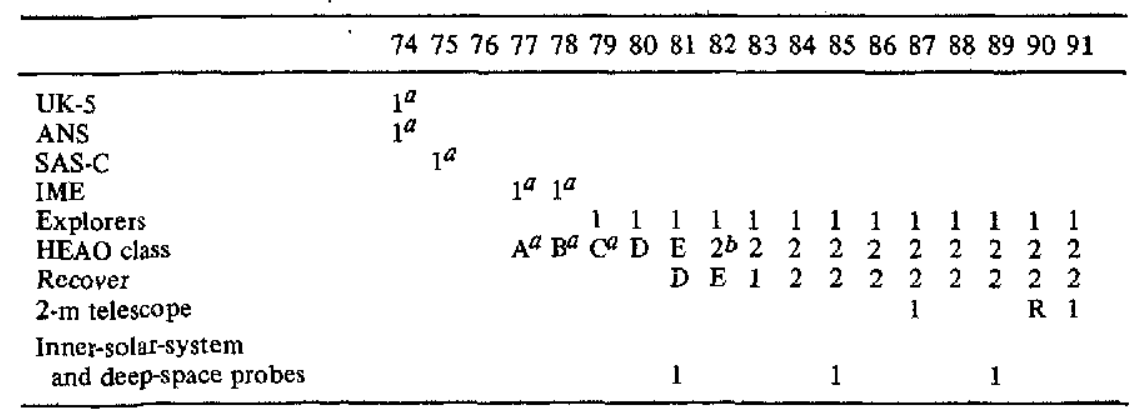

${ }^{a}$ Approved.

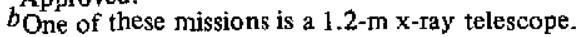


extent to which moderate-sized standardized spacecraft can be mass produced in an inexpensive manner. The decision on whether to recover and refurbish the Explorer spacecraft will be made on a case-by-case basis. Special requirements exist in the discipline for grazing-incidence $x$-ray telescopes and for deep-space probes. Assuming that HEAO-B is launched in 1978, the study group recommends replacing HEAO-B with a 1.2-m telescope in 1982, using the standard spacecraft. This telescope would be recovered in 1984, refurbished, and relaunched in 1985 . The 1.2-m telescope would be replaced by a $2-\mathrm{m}$ telescope in 1987, which would be serviced and recovered as required by the telescope technology in the late 1980's. Both telescopes would be operated as national $\mathrm{x}$-ray observatories. Long-baseline observations are required for x-ray, gamma-ray, and cosmic-ray observations. Deep-space and inner-solar-system probes are programmed for launch in 1981, 1985, and 1989 to meet these requirements. It is estimated that the above program can be carried out at an average cost of $\$ 120$ million $/ \mathrm{yr}$ to $\$ 150 \mathrm{million} / \mathrm{yr}$ in 1972 dollars.

\section{B. Sortie Mode}

The sortie mode can serve as a research platform to conduct shortterm observations and to test and check out new instruments prior to flight on free-flying spacecraft (see Table 8). It has been assumed that the balloon and sounding-rocket programs will continue into the

TABLE 8 High-Energy Astrophysics Mission Model-Sortie Model

747576777879808182838485868788899091

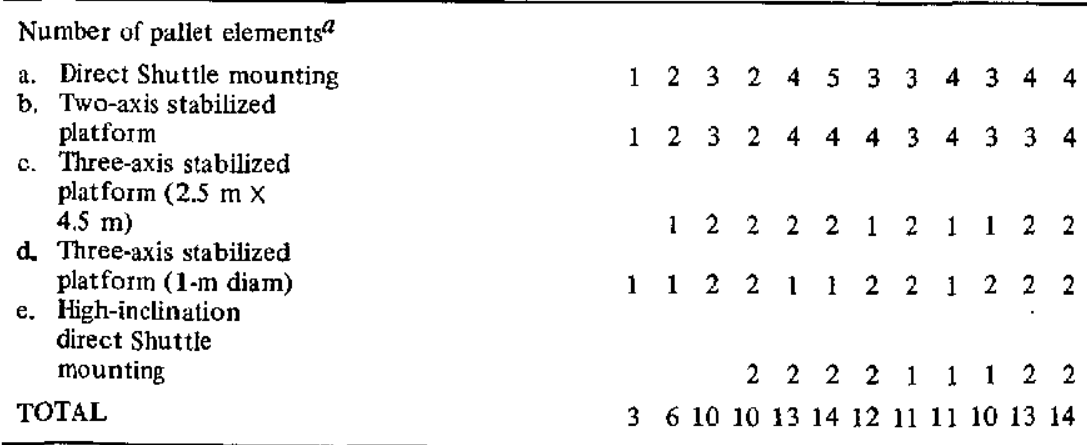

${ }^{a}$ A pallet element is assumed to be a structure $3 \mathrm{~m} \times 4.5 \mathrm{~m}$. 
Shuttle era. Shuttle pallet flights are planned to begin at a modest level in 1980 and will grow to a level of $10-15$ pallet element flights per year. The standard unit in the mission model is the $3 \mathrm{~m} \times 4.5 \mathrm{~m}$ pallet element. The working group found it possible to describe numerous $3-\mathrm{m}$ pallet size payloads, which are adequate to satisfy the needs of the discipline. Four types of stabilized platform are required. Shuttle flights at $28^{\circ}$ inclination and at high inclinations are required. It is estimated that a research program of $10-15$ pallet elements launched per year can be carried out at a cost of approximately $\$ 30$ million/yr in 1972 dollars.

\section{SUMMARY AND RECOMMENDATIONS}

The broad research area now called high-energy astrophysics is a most rapidly expanding field of modern astronomy, which is having a profound influence on astrophysics and fundamental physics and which requires instruments located above the earth's atmosphere. The discovery potential of this area is unique, as testified by the NAS Astronomy and Physics Survey Committees. Therefore, with respect to the program in high-energy astrophysics

1. We recommend that increased resources be devoted to this new and exciting area in order that the potential for discovery during the era of Shuttle operation be realized.

2 . We recommend that the major allocation of resources be given to free-flyers, i.e., large automated spacecraft for x-ray, gamma-ray, and cosmic-ray studies, since it is only on these missions that the long observing times and the continuity of observations that will be required for this discipline can be obtained.

3. We recommend that the HEAO program, which was recently considerably reduced, be continued and expanded, since it will extend naturally into the Shuttle era and forms the basis of our free-flyer concepts; that the developed and ready-for-construction instruments left over from the earlier HEAO program be implemented on either unmanned or Shuttle-launched missions in the late 1970's or very early 1980 's.

4. We recommend that an intermediate $\mathrm{x}$-ray telescope of at least 1.2-m aperture be launched in the early 1980's to be followed by the launch of a large $x$-ray telescope of at least 2-m aperture, both facilities to be operated as national observatories.

5. We recommend that space be allocated on inner-solar-system 
missions for experiments to measure solar cosmic rays and to provide long baselines for $\mathrm{x}$-ray and gamma-ray burst studies and that deepspace probes be implemented to study cosmic-ray phenomena outside the modulation of the solar magnetosphere.

We have devoted considerable effort to investigate methods by which the Shuttle opportunity can be used to obtain maximum science at minimum cost in this discipline area. We recognize that in addition to the large free-flyers there must be a range of opportunities from rockets and balloons to inexpensive single-experiment spacecraft. We regard the Shuttle sortie mode pallet as at least equivalent to a one-week or longer rocket or balloon flight with considerable enhanced capabilities. Therefore, with respect to high-energy astrophysics,

6. We recommend that the sortie be used to provide frequent inexpensive and rapid turnaround flight opportunities for a broad segment of the discipline. We believe that the best method of achieving this objective is to fly single 3-m pallet-sized elements often, rather than total missions dedicated to our discipline. This requires a new and simplified approach to management philosophy analogous to that now employed in the balloon and sounding-rocket programs.

7. We recommend that a standard support system for free-flyers similar to that available in the mini- HEAO program be defined. This support system could accommodate most of the high-energy astronomy experiments either as single instruments or in a multiexperiment bus.

8. We recommend that retrieval and return to earth of free-flyers is a possible valuable concept, which can materially reduce cost and increase flexibility, particularly with respect to national facilities. 


\section{5 \\ Infrared Astronomy}

\section{I N T RO D U C T I O N}

During the past decade, substantial contributions to astronomical knowledge have been made possible by rapid advances in infrared and millimeter-wave technology. These contributions have had an impact on the fields of solar-system physics, stellar formation and evolution, physics and chemistry of interstellar matter, galactic structure, and the origin of the universe. Infrared and submillimeter waves $(1 \mu \mathrm{m}$ to $1 \mathrm{~mm})$ are especially suited for the study of cool states of matter in the universe. Thermal radiation covering the temperature range from cool stars to the cosmic background radiation is predominantly in this spectral region. Infrared observations are also important for understanding the emission mechanism of nonthermal sources and for identifying and understanding the molecular constituents of astronomical objects.

Progress in infrared astronomy has been made in the past primarily by ground-based observations. Atmospheric absorption, however, limits these observations to narrow spectral windows; and even in these windows, atmospheric emission is substantial. Promising results have been obtained with small instruments flown on aircraft, balloons, and rockets, but aperture size and observing time have set severe limitations on these observations. The Space Shuttle will permit observations throughout the infrared and submillimeter spectral range without atmospheric interference and with considerably greater aperture, observing time, and sensitivity than would currently be possible on existing high-altitude platforms. In particular, orbital operation provides the unique circumstance of both low residual atmosphere and very low infrared sky radiance, making a large cryogenically cooled telescope both feasible and advantageous. Such an instrument is not possible on the ground and is of limited potential in the stratosphere. Operating in space, it can provide substantial gains 
in sensitivity relative to large ground-based instruments operating in the $10-\mu \mathrm{m}$ atmospheric window.

\section{I. S C I E N T I F I C O B J E C T I VES}

Several previous reports have addressed themselves to the subject of scientific goals and programs for infrared astronomy in considerable detail.* From these studies, it is clear that infrared astronomical observations are as crucial as are optical and radio observations to the understanding of nearly every fundamental question that can be posed in astronomy today. For the purpose of illustrating the potential of spaceborne infrared astronomy, a few examples are discussed briefly.

\section{A. Solar-System Formation}

High-resolution spectroscopy of planets and comets over the broad spectral regions that are available in space can give molecular abundances and hence atomic abundances and isotopic ratios for the solar system. This information provides a clue to the history of the objects within the solar system.

\section{B. Stellar Evolution}

Spectroscopy with the sensitivity available from instruments in space can provide measurements on the composition and structure of the interstellar medium, stellar atmospheres, and circumstellar gas. These measurements, together with those within the solar system mentioned above, are crucial to theories of nucleosynthesis, stellar atmospheres, and evolutionary models of stars.

\section{Galactic Structure and Evolution}

Maps of portions of the galaxy at $100 \mu \mathrm{m}$ with high spatial resolution, which can be made with a large cooled telescope, can define the distribution of gas and dust and its relation to the stellar

*A Long-Range Program in Space Astronomy, Position Paper of the Astronomy Missions Board, R. O. Doyle, ed., NASA SP 213 (National Aeronautics and Space Administration, Washington, D.C., 1969). Astronomy Survey Committee, Astronomy and Astrophysics for the 1970's, Vol. 2, Reports of the Panels (National Academy of Sciences, Washington, D.C., 1973). 
content in the galaxy. Sensitive long-wavelength photometry will be able to test theories of the mechanisms that generate the enormous infrared luminosities of many galaxies and cosmological objects.

\section{Cosmology}

It is of fundamental importance to understand fully the cosmic background radiation, as it may well be a remnant of the hot early phase of our universe. Data from ground-based observations and balloon and rocket flights are consistent with this interpretation, but what is now required is a good measurement of the short-wavelength portion of the spectrum, which can only be made from outside the earth's atmosphere with narrow-band instruments. Observation of any spatial or spectral anisotropies in this radiation would have great cosmological significance.

\section{I I. SHUT TLE TELES COPES: EVOLUTIONARY APPROACH}

The primary aim of infrared space astronomy is to set up an observatory in space. Existing technology is adequate to commence immediately with the design and construction of two classes of telescopes capable of making ir observations from space, one to operate at ambient temperature and the other to be cryogenically cooled. Each of these types of instruments is peculiarly suited to specific types of measurement, and both can be used to great advantage for ir observations outside the atmosphere. The type of instrumentation at the focal planes can be changed, depending on the particular scientific goal in mind, just as at a ground-based facility.

It is intended that these general-purpose telescopes be flown early in the program. A spatial interferometer for obtaining high angular resolution at long infrared wavelengths is proposed for later in the program. This system could incorporate two or more $1-\mathrm{m}$ ambienttemperature telescopes.

These instruments are the first phase in an evolutionary program. With the experience gained, the design of larger instruments with collecting areas an order of magnitude greater can begin. A $2.5-\mathrm{m}$ cryogenically cooled telescope is probably the largest that can be used on the Shuttle, but one can consider the practicality of constructing an even larger ambient-temperature telescope $(10 \mathrm{~m})$ in space. 
TABLE 9 Evolutionary Stages in the Development of Observational-Type Infrared Instruments

\begin{tabular}{llll}
\hline & Early 1980's & Mid-1980's & Late 1980's \\
\hline Evolutionary stage & & & \\
Cryogenically cooled telescope & $1 \mathrm{~m}$ & & $2.5 \mathrm{~m}$ \\
Ambient-temperature telescope & $1 \mathrm{~m}$ & $3-\mathrm{m}$ (LST) & $10 \mathrm{~m}$ \\
Spatial interferometer & & $10-\mathrm{m}$ base & $1-\mathrm{km}$ base \\
\hline
\end{tabular}

A similar development is proposed for the spatial interferometer. Initially it will operate with a $10-\mathrm{m}$ baseline on the Shuttle, but this will be extended to much longer baselines between the Shuttle and an associated free-flyer.

The evolutionary approach is shown in Table 9. It is important to note that the early instruments will continue to be useful throughout the Shuttle period for investigations that do not require the higher performance of the later systems. In particular, a $1-\mathrm{m}$ ambient telescope can be used with the spatial interferometer.

During the early Shuttle period it will be important to launch free-flyers with specific objectives such as the measurement of the cosmic background and its isotropy as well as an exploratory survey mission. Also, it is anticipated that throughout the Shuttle period smaller instruments of the "hitchhiker" class, which use rocket-type systems, are needed to test new ideas and instrumentation.

\section{A. Cryogenically Cooled Telescopes}

Ground-based telescopes utilizing the best broadband infrared detectors available are presently limited by background noise from the thermal emission of the telescope and atmosphere. Beamswitching techniques, which provide discrimination between a faint discrete source and a bright uniform background cannot overcome this fundamental limitation. Full utilization of current and anticipated broadband detector technology requires the use of a cryogenically cooled telescope operating above the atmosphere. Such an instrument would be optimum for broadband photometry and multiplex spectroscopy of faint galactic and extragalactic sources. In addition, a cryogenic telescope would make possible wide-angle (large-beam) photometry, permitting the mapping of very low surface-brightness extended sources and absolute flux measurements using total field chopping. 
The limiting performance for a Shuttleborne $(1.5-\mathrm{m})$ cryogenically cooled telescope is given in Table 10 .

We recommend two instruments in this category. The first is a 1to $1.5-\mathrm{m}$ telescope, which can be constructed largely with existing technology. The second-a long-range goal-is a $2.5-\mathrm{m}$ telescope whose design will be based on experience with the smaller telescope. The characteristics of these two instruments are given in Table 11.

The temperature to which the telescope should be cooled for broadband diffraction-limited operation depends on the anticipated detector noise equivalent power (NEP), detector quantum efficiency, detector size, wavelength interval, temperature of the detector cavity, and telescope emissivity. Broadband detectors with an NEP of $10^{-16} \mathrm{~W} \mathrm{~m}^{-2} \mathrm{~Hz}^{-\frac{1}{2}}$ are currently available for use between 10 and $30 \mu \mathrm{m}$. Comparable detectors should be achieved at longer wavelengths by the 1980's. To take full advantage of such a detector in the 10 - to $20-\mu \mathrm{m}$ region requires reducing the background radiation

TABLE 10 Limiting Performance of a 1.5-m $\mathrm{LH}_{2}$-Cooled Infrared Telescope $e^{a, b}$

\begin{tabular}{|c|c|c|c|c|c|c|}
\hline Object & $\begin{array}{l}\lambda \\
(\mu \mathrm{m})\end{array}$ & $\begin{array}{l}\theta \\
\text { ( } \min \text { of } \\
\text { arc) }\end{array}$ & $\begin{array}{l}D \\
(\mathrm{pc})^{c}\end{array}$ & $\begin{array}{l}\lambda F_{\lambda} \\
\left(\mathrm{W}^{-2}\right)^{c} \\
\left.\mathrm{~m}^{-2}\right)^{2}\end{array}$ & $\begin{array}{l}D_{m} \\
(\mathrm{pc})^{c}\end{array}$ & $\begin{array}{l}D_{\theta} \\
(\mathrm{pc})^{\mathrm{c}}\end{array}$ \\
\hline a Orionis & $\begin{array}{r}.10 \\
100\end{array}$ & & 450 & $\begin{array}{l}1(-9) \\
1(-12)\end{array}$ & $\begin{array}{l}13(5) \\
10(3)\end{array}$ & \\
\hline $\begin{array}{l}\text { Orion nebula } \\
\text { Kleinmann-Low nebula }\end{array}$ & $\begin{array}{r}100 \\
10 \\
100 \\
350\end{array}$ & $\begin{array}{l}6 \\
0.5 \\
1 \\
1\end{array}$ & $\begin{array}{l}450 \\
450\end{array}$ & $\begin{array}{c}1(-8) \\
25(-11) \\
5(-9) \\
4(-11)\end{array}$ & $\begin{array}{r}1(6) \\
7(5) \\
7(5) \\
70(3)\end{array}$ & $\begin{array}{l}10(3) \\
80(3) \\
15(2) \\
15(2)\end{array}$ \\
\hline Sagittarius B2 & $\begin{array}{l}100 \\
350\end{array}$ & $\begin{array}{l}2 \\
2\end{array}$ & $10(3)$ & $\begin{array}{l}4(-9) \\
6(-11)\end{array}$ & $\begin{array}{l}13(6) \\
17(5)\end{array}$ & $\begin{array}{l}50(3) \\
15(3)\end{array}$ \\
\hline Galactic center & $\begin{array}{r}10 \\
100 \\
100\end{array}$ & $\begin{array}{c}0.4 \\
5 \\
390\end{array}$ & $10(3)$ & $\begin{array}{c}15(-11) \\
2(-9) \\
1(-7)\end{array}$ & $\begin{array}{r}1(6) \\
10(6) \\
70(6)\end{array}$ & $\begin{array}{r}150(3) \\
200(3) \\
15(6)\end{array}$ \\
\hline M82 & $\begin{array}{r}10 \\
100\end{array}$ & $\begin{array}{l}0.5 \\
0.5\end{array}$ & $32(5)$ & $\begin{array}{l}8(-12) \\
6(-11)\end{array}$ & $\begin{array}{l}7(8) \\
7(8)\end{array}$ & $\begin{array}{r}60(6) \\
6(6)\end{array}$ \\
\hline $\begin{array}{l}\text { NGC } 1068 \\
3 \mathrm{C} 273\end{array}$ & $\begin{array}{l}10 \\
10\end{array}$ & 0.02 & $\begin{array}{l}17(6) \\
12(8)\end{array}$ & $\begin{array}{r}75(-13) \\
9(-14)\end{array}$ & $\begin{array}{r}4(9) \\
30(9)\end{array}$ & \\
\hline
\end{tabular}

${ }_{b}^{a}$ From Volume I of the Final Report of the NASA Shuttle Payload Working Groups.

${ }^{b} D$ is the distance of the objects that have been observed in the infrared.

$D_{m}$ is the distance at which similar objects could be observed with a $1.5-\mathrm{m}$ cooled telescope. $D_{\theta}$ is the maximum distance beyond which the extended objects shown, of angular diameter $\theta$, could not be spatially resolved with this telescope. The flux levels actually observed for these objects are denoted by $\lambda F_{\lambda}$. The calculations of $D_{m}$ assume a signal-to-noise ratio of 1 and a 1-sec integration time. Detector NEP is taken as $10^{-16} \mathrm{~W} \mathrm{~m}^{-2} \mathrm{~Hz}^{-1 / 2}$ at $10 \mu \mathrm{m}$ and 2 $\times 10^{-15} \mathrm{~W} \mathrm{~m}^{-2} \mathrm{~Hz}^{-1 / 2}$ at $100 \mu \mathrm{m}$ with assumed transmission ef ficiency 0.44 .

$c_{\text {Numbers in parentheses are powers of } 10 .}$ 
flux approximately four orders of magnitude below that which is encountered in ground-based observations. The greatest gain comes from cooling the telescope to approximately $20 \mathrm{~K}$ (liquid hydrogen temperature). The cryogenic telescope should be constructed with provision for more than one instrument that can be mechanically switched into the focal plane. Such instruments could include a multiband photometer, imaging devices, and a multiplex spectrometer. The detectors would be cooled to 2 to $4 \mathrm{~K}$ by liquid helium.

The 1-m cryogenic telescope is well suited to a sortie payload by its developmental nature, its cryogenic requirements, and the desire to update frequently the instruments at the focal plane. It should be fully gimbaled. Special attention must be paid to baffling against radiation from the earth, sun, and moon and the Shuttle bay radiators. A baffle extending outside the Shuttle bay will be needed to provide rejection of off-axis radiation. A field of view of approximately $15 \mathrm{~min}$ of arc is required to permit fine acquisition and position objects in the focal plane and to allow for multiple detectors and imaging devices.

The cooled telescope is to be the prime experiment on the particular flight because of pointing demands and weight.

Attention must be paid to the problem that contamination presents for the cryogenically cooled telescope. The low operating tem-

TABLE 11 Cryogenically Cooled Telescopes

\begin{tabular}{|c|c|c|}
\hline Mode & $\begin{array}{l}\text { A } \\
(1980-1985) \\
\text { Sortie }\end{array}$ & $\begin{array}{l}\mathrm{B} \\
(1985-1990) \\
\text { Sortie or Free-flyer }\end{array}$ \\
\hline Diameter of primary $(\mathrm{m})$ & ] & 2.5 \\
\hline Effective area $\left(\mathrm{m}^{2}\right)$ & 0.7 & 4.2 \\
\hline Diffraction image size at $\quad 5 \mu \mathrm{m}$ (sec of arc) & 2.5 & 1 \\
\hline $10 \mu \mathrm{m}(\sec$ of arc $)$ & $s$ & 2 \\
\hline $100 \mu \mathrm{m}(\mathrm{sec}$ of $\mathrm{arc})$ & 50 & 20 \\
\hline Field of view ( $\min$ of arc) & 15 & 5 \\
\hline Pointing stability (sec of arc) & 0.5 & 0.2 \\
\hline Operating temperature (K) & 20 & 20 \\
\hline Detector temperature $(\mathbf{K})$ & $2-4$ & $2-4$ \\
\hline Weight $(\mathrm{kg})$ & 5000 & $\sim 15,000$ \\
\hline Power $(\mathrm{kW})$ & 2 & \\
\hline Operation & From ground or cabin & \\
\hline Data rate $(\mathrm{kHz})$ & $<25$ & \\
\hline Mount & Fully gimbaled & $\begin{array}{l}\text { Gimbaled or } \\
\text { frec-flying }\end{array}$ \\
\hline
\end{tabular}


perature is sufficient to freeze out the residual atmospheric gas, as well as outgassing and leakage from the Shuttle. Also, particulate matter, or "dust," can be a serious problem by increasing the background radiation to the point of degrading the instrumental performance and making the total-field-chopping mode of data taking unsatisfactory. Further discussion of the contamination problem is in Section V.D.

\section{B. Ambient-Temperature Telescopes}

Cryogenic cooling $(<20 \mathrm{~K})$ is highly advantageous whenever thermal emission from the telescope is the major limitation in reaching ultimate sensitivity, as discussed in Section III.A. When other noise sources dominate, emission from the telescope cooling yields negligible improvement. This is true for the following two cases:

1. Spectroscopy in very narrow spectral intervals throughout the infrared and submillimeter region where detector noise generally dominates telescope background noise within the narrow interval.

2. Studies of relatively bright sources, such as the planets and other objects in the solar system, where the source dominates emission from the telescope.

In both cases observations can be made more efficiently and at lower cost with an uncooled telescope. Independent of the question of telescope emission, investigations that require a high angular resolution must, for the foreseeable future, take advantage of the larger apertures $(3 \mathrm{~m}, 10 \mathrm{~m})$ feasible with uncooled telescopes. A cooled telescope with $10-\mathrm{m}$ aperture, although desirable from a scientific point of view, seems not to be feasible from an economic point of view in the 1980's.

The 3-m telescope of the Large Space Telescope (LST) mission can provide many of the capabilities needed for infrared observations. The potential available time on the LST might, however, not be adequate to satisfy the needs of the infrared community. In addition, the LST will not be optimized for low emissivity in the infrared. We therefore consider, as part of an evolutionary approach, ambienttemperature telescopes optimized specifically for infrared and submillimeter-wave operation. If sufficient utilization can be made of the LST capabilities, the easy steps in this approach can be leapfrogged. 


\section{ONE-METER AMBIENT-TEMPERATURE TELESCOPE}

The first telescope in this category is a 1-m telescope optimized specifically for infrared and submillimeter-wave operation. Optimization consists of elimination or at least substantial reduction of emission from baffles, support structure of the secondary, and the mirror surfaces themselves. Careful design should achieve a telescope emissivity of not more than 0.01 or 0.02 .

The $1-\mathrm{m}$ telescope is viewed as an early Shuttle sortie payload, possibly beginning operation during the Shuttle test flights. The moderate size and weight of the telescope will allow operation on a nondedicated mission and is well suited to gain operational experience with pointed astronomical instruments in the sortie mode. Furthermore, experience with this telescope is expected to be valuable for the design and operation of later, larger ambient temperature instruments. This telescope will yield higher sensitivity than can be obtained from stratospheric balloon and aircraft telescopes of similar size.

In the late 1980's this telescope could be used as one element of a spatial interferometer as discussed in Section IV.C. The characteristics of the 1-m ambient-temperature telescope are given in Table 12.

\section{THREE-METER AMBIENT-TEMPERATURE TELESCOPE}

The second stage in the evolutionary process of uncooled infrared telescopes is an instrument in the 3-m class, which represents an order of magnitude increase in the collecting area over the $1-\mathrm{m}$ unit. This telescope should again be of a low-emissivity design optimized for operation at infrared and millimeter waves and diffraction limited at $5 \mu \mathrm{m}$. The characteristics of this telescope are also given in Table 12.

While not optimized for infrared observations, regular use of the LST with cryogenically cooled detectors could provide some of the capability of the 3-m infrared telescope in terms of large aperture for narrow-band spectroscopy and spatial resolution of bright sources throughout the infrared and submillimeter region. It will also be possible to take advantage of the high optical quality of the LST to obtain sensitivity and resolution superior to what can be done from the ground by using efficient imaging detectors that should be available in the next decade for the near infrared. 
TABLE 12 Ambient-Temperature Telescope

\begin{tabular}{|c|c|c|c|}
\hline Parameter & $\begin{array}{l}\text { A } \\
(1979)\end{array}$ & (1980-1985) & $\begin{array}{l}C \\
(1985-1990)\end{array}$ \\
\hline Mode & Sortie & $\begin{array}{l}\text { Sortie or } \\
\text { free-flyer }\end{array}$ & $\begin{array}{l}\text { Construction of } \\
\text { free+flyer }\end{array}$ \\
\hline Diameter of primary (m) & 1 & 3 & 10 \\
\hline Effective area $\left(\mathrm{m}^{2}\right)$ & 0.64 & 6 & 64 \\
\hline Diffraction limited at wavelength $(\mu \mathrm{m})$ & 1 & 5 & 20 \\
\hline Image size at $\quad 1 \mu \mathrm{m}$ ( $\mathrm{sec}$ of arc) & 0.5 & 0.8 & 1 \\
\hline \multirow{2}{*}{ (2.5 $\mathrm{N} / \mathrm{D}$ when applicable) $\begin{array}{r}10 \mu \mathrm{m} \text { (sec of arc) } \\
100 \mu \mathrm{m} \text { (sec of arc) }\end{array}$} & 5 & 1.7 & 1 \\
\hline & so & 17 & 5 \\
\hline Field of view ( $\min$ of arc) & -40 & & 20 \\
\hline Pointing stability (sec of arc) & 0.2 & 0.1 & 0.1 \\
\hline Operating temperature of primary $(K)$ & $200-250$ & $150-200$ & $150-200$ \\
\hline Weight $\{\mathrm{kg}\}$ & -1000 & $\sim 10,000$ & $\sim 25,000$ \\
\hline Emissivity (lelescope and mirror) & 0.01 & 0.01 & 0.01 \\
\hline Detector temperature $(\mathrm{K})$ & $2-10$ & $2-10$ & $2-10$ \\
\hline Power ( $\left.W_{\mathrm{av}}\right)$ & $\sim 100$ & & \\
\hline Operation & From ground or cabin & & \\
\hline Data rate $(\mathrm{kHz})$ & $\sim 100$ & & \\
\hline Mount & Fully gimbaled & $\begin{array}{l}\text { Gimbaled or } \\
\text { free-flying }\end{array}$ & Free-flying \\
\hline
\end{tabular}

\section{TEN-METER-DIAMETER AMBIENT-TEMPERATURE TELESCOPE}

The long-range goal in the development of ambient-temperature telescopes is a very large instrument that would provide the collecting area needed for high-resolution spectral studies and the aperture for good spatial resolution. The characteristics of a $10-\mathrm{m}$ instrument are given in Table 11. This instrument is diffraction limited at $20 \mu \mathrm{m}$ with an image of $1 \mathrm{sec}$ of arc.

The 10-m telescope represents a new mode of Shuttle use because the telescope must be assembled in orbit. The telescope might be deployed by unfolding an array of rigid segments, the relative orientation of the segments to be determined by a set of laser beams. More than one Shuttle flight might be required to assemble and test the system. Thereafter, regular visits will be required for replacing detector cryogenic supplies, changing instrumentation, and maintenance.

A high orbital inclination, synchronous in a 6 a.m.- 6 p.m. orbit, seems desirable from the viewpoint of solar power and thermal control. Thermal control and baffling need very careful considerations in order to achieve reasonable low mirror temperatures by passive means, as well as a low emissivity. Many control and orientation subsystems can be adapted from the LST. 


\section{V. SPECIALIZED I NSTR UMENT S}

For certain measurements the observatory type of telescone is not suitable and specialized instruments are required. Instruments in this class are not envisaged as always being major undertakings but should also include relatively small pieces of equipment that individual groups with modest budgets could undertake to develop and construct. This point is particularly important since it significantly enlarges the community of astronomers involved in the Shuttle program and greatly increases the chance for new and exciting science.

Examples are given in this section of the type of specialized instrumentation for space use that can have an impact on infrared astronomy in the 1980's.

\section{A. Cosmic Background Radiation}

The $3 \mathrm{~K}$ cosmic background radiation affords astronomers a unique opportunity to study a fundamental problem. Considerable effort should be devoted to make accurate measurements of this radiation, both spectrally and spatially.

A critical test of the blackbody nature of the radiation is to determine the spectrum on the short-wavelength side of the peak. This is probably best done with a free-flying satellite containing a cooled spectrometer of moderate resolution.

The same satellite should also be capable of investigating the extent to which the background is isotropic. This is of great cosmological interest, as it gives information on the structure of our universe and its past history. Measurements of sufficient accuracy would enable our velocity to be determined relative to the frame in which the radiation is isotropic. Anisotropies can also arise from many other causes such as density fluctuations in the universe caused, for example, by clustering of OSO's. These measurements require the ability to point the satellite and to be able to detect small relative temperature differences of the order of 0.1 percent. The long integration times necessary will be possible with a free-flyer.

\section{B. Discrete-Source Sky Survey, Infrared Monitor}

There is a requirement for a free-flying cooled telescope to make an unbiased survey of infrared sources on the celestial sphere. This would complement and improve upon the rather selective and limited scans possible from aircraft, rockets, and balloons and would 
identify objects of interest for further study using the larger telescopes on the Shuttle. In addition, and of great importance, the same instrument can be used to monitor changes of intensity of selected sources by repetitive scanning; the variability of sources could thus be followed continuously.

\section{Spatial Interferometer}

To obtain spatial resolution in the far infrared that is comparable with that obtained at optical and radio wavelengths, it is necessary to increase the size of the collecting optics in proportion to the wavelength. The largest size contemplated for a conventional telescope in the far infrared is the 10-m Shuttle telescope, which would give an angular resolution $(2.5 \lambda / D)$ of $5 \mathrm{sec}$ of arc at $100 \mu \mathrm{m}$. Better angular resolution than this is required for many astronomical problems, such as the separation of the various components of complex $\mathrm{H}$ II regions and the determination of infrared sizes of objects. This improved resolution can probably best be achieved by the use of a spatial interferometer operating over a relatively long baseline (as in the radio region). Such an instrument will rely heavily on the progress made during the next few years in the development of tunable lasers and coherent detection techniques.

In addition, there is the problem of determining the parameters of the baseline to the required accuracy. It is proposed that initially both telescopes $(1 \mathrm{~m})$ be mounted in the Shuttle, giving an angular resolution similar to a $10-\mathrm{m}$ telescope. This angular resolution would later be improved by operating with one or both of the $1-\mathrm{m}$ telescopes mounted in associated free-flyers. The extent to which the resolution can be improved depends on how long a baseline it is practical to use-two $1-\mathrm{m}$ dishes operating $1 \mathrm{~km}$ apart would give an angular resolution of the order of hundredths of seconds of arc, i.e., two orders of magnitude improvement over a conventional telescope.

\section{REQUIREMENTS ON THE SHUTTLE CA P A B I LI T Y}

\section{A. Sortie Mode}

All instruments considered for the sortie mode will be mounted on the pallet. The Shuttle should provide a mechanical interface, electrical power, control links from the Shuttle cabin, data storage and handling facilities, and communication with the ground. 
Operation of the instruments, including guidance, will be done remotely either from the ground or by the payload specialist. Manned access to the infrared detectors on the telescopes is not required and would be extremely difficult, as the detectors are cryogenically cooled, even on the ambient-temperature telescope.

Although man's presence in the Shuttle can be used to advantage, it does not appear necessary to have a large crew. Missions up to 30 days offer the greatest rewards for ir astronomy, and large crews will decrease payload weight and mission length. Much of the telescope operation, and virtually all of the data analysis and decision making, can be done best by a ground-based team of scientists who have ready access to libraries and computers. Acquisition of predetermined objects could be accomplished most efficiently by a computer using continuous readings on Shuttle attitude and position relative to earth and sun. These considerations suggest that only the minimum crew of two pilots, a mission specialist, and a payload specialist be used for ir astronomy sortie missions. There is adequate room in the Shuttle cabin for the payload control console, display units, and some data analysis and storage equipment. This appears to eliminate the need for an additional pressurized cabin with its serious weight penalty.

The use of the payload specialist in the Shuttle cabin requires that provisions exist for electrical interfaces between the payload specialist's console and the pallet-mounted telescope. In addition to two or three display panels, a control panel, and other electronics associated with the scientific instruments, a small computer and tape recorder will be required for operating the telescope and for data handling. These instruments must interface with the Shuttle telemetry system as well as with the telescope.

Tentative values for a number of parameters that make demands on Shuttle capability are tabulated below for the case of the $1-\mathrm{m}$ cooled telescope:

Orbital Altitude: $400 \mathrm{~km}$. This is a compromise between the need to stay below the Van Allen belts to minimize highenergy radiation noise on the detectors and the need to minimize infrared radiation from the overlying earth atmosphere.

Spacecraft Attitude: The Shuttle bay must be pointed away from the earth. This is required both to permit viewing of celestial objects and to reduce the number of constraints on telescope viewing angle produced by thermal radiation into the telescope barrel from the earth and the Shuttle bay doors. 
Flight Frequency ( 2 per year): A greater frequency is desirable. If the frequency is less than two per year it may become difficult to justify the investment in money and scientists' time required to develop the facilities.

Duration in Orbit: 7 to 30 days, as long as possible.

Telescope Weight Estimate $(5000 \mathrm{~kg})$ : This includes the stabilization and acquisition systems, the Dewars, the control electronics, the optics and their support structure, baffling, and the entire complement of attached instruments. The weight could be decreased considerably at increased cost in materials and engineering.

Telescope Volume Estimate: $16 \mathrm{~m}^{3}$.

Shuttle Stabilization Limits $\left( \pm 5^{\circ}\right)$ : Note that the telescope gimbal system is assumed to provide stabilization to $0.5 \mathrm{sec}$ of arc and pointing capability over a large fraction of a hemisphere.

Unobstructed Viewing Angles: At least $90^{\circ}$ in the plane of the long axis of the Shuttle and its tail and at least $120^{\circ}$ in the plane perpendicular to the long axis. The later requirement permits $30 \mathrm{~min}$ of continuous viewing of objects near the celestial equator without slewing the Shuttle if the Shuttle has its long axis perpendicular to the orbital plane.

Electrical Power ( $2 \mathrm{~kW}$, continuous): This would supply the telescope its instrument complement, the stabilization and pointing system, the $32 \mathrm{~K}$ word computer, tape recorder, and other payload electronics.

Communications: Continuous voice up and down plus a command link up and data link ( $25 \mathrm{kbits} / \mathrm{sec}$ or more) down. Short periods of $200 \mathrm{kbits} / \mathrm{sec}$ down are also required.

The requirements of the $1-\mathrm{m}$ ambient temperature telescope are similar but less demanding in weight, volume, and power, as indicated in Table 11.

If the $2.5-\mathrm{m}$ cooled telescope is used in the sortie mode, its weight, power, and volume requirements on the Shuttle obviously would be much greater. It would occupy the full diameter of the Shuttle bay for a length of at least $12 \mathrm{~m}$. Its weight and power would be determined from tradeoffs between Shuttle capacity and telescope cost and performance.

The 10-m baseline spatial interferometer has the additional requirement that the two components will be placed $10 \mathrm{~m}$ apart in the Shuttle bay. The $1.5-\mathrm{km}$ baseline spatial interferometer, still in a very early conceptual phase, will require that the Shuttle orbit approximately $1.5 \mathrm{~km}$ from a subsatellite that it launches early in the mission. The subsatellite must always be visible from the payload bay. 
These requirements will probably demand more maneuvering of the Shuttle by the astronauts than most other sortie-mode experiments.

\section{B. Launch Mode}

In this mode, the Shuttle is used as a checkout and launch facility. Payloads with instruments operating in the infrared and submillimeter wavelengths will range in size from small rocket-type freeflyers to large planetary probes requiring Tug or other additional propulsion units. The role of man will be primarily to check out the payloads prior to their separation from the Shuttle. Man will also maneuver the Shuttle and deployment mechanisms during payload separation from the Shuttle.

\section{Assembly Mode}

By the late 1980's the need for a telescope with a primary mirror larger than the Shuttle bay diameter will require construction, assembly, or deployment of a large structure in orbit. New techniques such as laser alignment of individual elements will be necessary. Although most of the mechanical deployment will have to be automatic, it is expected that a substantial crew, perhaps as many as eight, will be required. Revisits to the telescope may be needed.

\section{Contamination}

One of the principal advantages of infrared observations from space is the elimination of the most disturbing effects of the earth's atmosphere. The full potential sensitivity of the space environment can be realized only if sufficient care is given to the effects of the residual atmosphere or the contamination by the spacecraft. The sources of contamination that have to be considered are (1) residual atmosphere; (2) spacecraft loutgassing, reaction control subsystem (RCS) firings, dumps, and dust emission] ; (3) high-energy radiation (Van Allen radiation belts, South Atlantic Anomaly).

\section{THE RESIDUAL ATMOSPHERE}

The large cryogenically cooled telescope imposes the most severe requirements on contaminants. Because the temperature of the telescope is expected to be $\sim 20 \mathrm{~K}$, major atmospheric constituents can 
condense on optical surfaces. For low orbits, the atomic and molecular gas densities will be approximately $10^{9} / \mathrm{cm}^{3}$. Therefore a maximum rate of condensation, of one monolayer every $10 \mathrm{sec}$, will occur when the telescope is pointed in the direction of motion. In this case, a sortie mission of approximately $10^{6} \mathrm{sec}$ will deposit $10^{5}$ monolayers, which would severely impair the telescope performance. The rates will be substantially less when viewing normal to the direction of motion. However, more detailed studies of this problem will be required in conjunction with the design of a specific instrument.

\section{CONTAMINATION INTRODUCED BY THE SPACECRAFT}

All contaminants introduced by the spacecraft should be kept below the level of the residual atmosphere. The outgassing and leakage from the Shuttle is a potential source of contamination for cryogenic optics; there will also be infrared emission from the warm gas. Apollo 15 data indicate that a water leakage and outgassing rate of $3 \times 10^{-4}$ $\mathrm{g} \mathrm{sec}^{-1}$ produced a return rate of $\sim 10^{12} \mathrm{~cm}^{-2} \mathrm{sec}^{-1} \mathrm{sr}^{-1}$, with other gases 100 times more abundant. If the telescope shield reduces the solid angle to $10^{-2} \mathrm{sr}$, the rate of deposition of all leakage gases is $\sim 1$ monolayer/h, which may be tolerable for the proposed Shuttle missions.

The 25-lb RCS used for attitude control will generate gaseous products at a mean rate of $0.3 \mathrm{~g} / \mathrm{sec}$, which may be some hundred times higher than leakage from the Shuttle. Therefore these systems must be designed to have a return rate of less than 1 percent to the vicinity of the spacecraft to avoid significant increase in contamination.

All waste should be stored until the end of the mission.

Particulate emission from the Shuttle is an important consideration for infrared missions. For example, a single dust particle of $10-\mu \mathrm{m}$ diameter at ambient temperature is readily detectable up to tens of kilometers with the sensitive systems envisaged (NEP $\sim W^{-1 / 2}$ ). If the rate of emission of particles larger than $10 \mu \mathrm{m}$ is $\sim 10^{-4} \mathrm{~cm}^{-2} \mathrm{sec}^{-1}$, there will be, on the average, one particle in the beam of the $1-\mathrm{m}$ telescope at any time. Therefore it is important to determine how dust emissions can be reduced.

Unwanted infrared radiation scattered into the telescope from sources such as the sun, the earth, the moon, and the Shuttle itself must be reduced by appropriate baffling. 
TABLE 13 Preliminary Acceptable Contamination Levels for Infrared Astronomy Sortie Missions

\begin{tabular}{ll}
\hline Return rate (due to leakage and outgassing) & $\leq 10^{12}$ molecules $\mathrm{cm}^{-2} \mathrm{sec}^{-1}$ \\
Particulate emission (10 $\mu \mathrm{m}$ or larger) & $<10^{-4} \mathrm{~cm}^{-2} \mathrm{sec}^{-1}$ \\
RCS fuel expended (1\% return) & $<20 \mathrm{~kg} \mathrm{day}$ \\
Waste dumps & None \\
$\begin{array}{l}\text { Column density of } \mathrm{H}_{2} \mathrm{O} \text { etc. } \\
\quad \text { (molecules with dipole moments) }\end{array}$ & $<10^{10}-10^{12} \mathrm{~cm}^{-2}$ \\
Background ionizing radiations & $<$ one event per sq cm per sec \\
& in region of the detector \\
\hline
\end{tabular}

Any discussion of contamination of Shuttle-based infrared astronomy depends on the design of the spacecraft and the state of the art in detector and instrument design in the 1980's. Preliminary discussions such as this are sufficient to demonstrate that a problem exists. We, therefore, recommend that NASA set up a group of working astronomers and engineers to study this problem in detail and to follow the evolution of Shuttle design and infrared technology so that serious conflicts do not arise.

\section{HIGH.ENERGY RADIATION}

The photoconductors used for detection of infrared radiation are also sensitive to high-energy radiation. Regions such as the Van Allen belts and the South Atlantic Anomaly, where the density of this radiation is high, are to be avoided.

Contamination level requirements are summarized in Table 13.

\section{POTENTIAL MISSION MODEL}

Table 14 gives a potential mission model for the infrared and submillimeter instruments described in this report.

\section{SUPPORTING RESEARCH AND TECHNOLOG Y}

Orbiting cryogenic telescopes will offer an unparalleled opportunity for astronomical observations in the infrared. The large technological effort to construct and orbit an infrared observatory must be balanced by a substantial scientific supporting research and technology (SR\&T) effort to ensure maximum effectiveness of an overall infrared program. A vigorous program of infrared astronomy should be 
TABLE 14 Infrared Astronomy Launch Schedule

\begin{tabular}{|c|c|c|c|c|c|c|c|c|c|c|c|c|c|}
\hline & \multicolumn{13}{|c|}{ Year } \\
\hline & 79 & 80 & 81 & 82 & 83 & 84 & 85 & 86 & 87 & 88 & 89 & 90 & 91 \\
\hline \multicolumn{14}{|l|}{ Sortic } \\
\hline $\mathrm{LH}_{2}$-cooled, $1 \mathrm{~m}$ & & & $1 / 2$ & $2 \times 1 / 2$ & $2 \times 1 / 2$ & $2 \times 1 / 2$ & $2 \times 1 / 2$ & $1 / 2$ & $1 / 2$ & & & & \\
\hline Ambient-temperature, $1 \mathrm{~m}^{a}$ & $1 / 4$ & $2 \times 1 / 4$ & $1 / 4$ & $2 \times 1 / 4$ & $2 \times 1 / 4$ & $2 \times 1 / 4$ & $2 \times 1 / 4$ & & & & & & \\
\hline Ambient-temperature, $3 \mathrm{~m}^{b}$ & & & & & 1 & 1 & 1 & 1 & 1 & 1 & & & \\
\hline Interferometer $10-\mathrm{m}$ baseline & & & & & & & & $1 / 2$ & $1 / 2$ & & & & \\
\hline $\mathrm{LH}_{2}$-cooled $2.5 \mathrm{~m}^{b}$ & & & & & & & & & 1 & 2 & 2 & 2 & 2 \\
\hline Interferometer, $1.5-\mathrm{km}$ baseline $\mathrm{e}^{b}$ & & & & & & & & & & $1 / 2$ & $1 / 2$ & $1 / 2$ & $1 / 2$ \\
\hline Ambient-temperature, $10 \mathrm{~m}^{c}$ & & & & & & & 1 & & & & 1 & $1 / 4$ & $1 / 4$ \\
\hline \multicolumn{14}{|l|}{ Small Free-Flyers and Rocket Payloads } \\
\hline Ir survey & & & $\mathrm{x}$ & & & $\mathrm{x}$ & & & & & & & \\
\hline Submillimeter background & & & & $\mathrm{x}$ & & & $\mathbf{x}$ & & & & & & \\
\hline Millimeter background & & & & & $\mathrm{x}$ & & & $\mathrm{x}$ & & & & & \\
\hline Ir monitors/Explorers & & & & & & $\mathrm{x}$ & & & $\mathrm{x}$ & $\mathrm{x}$ & $\mathrm{x}$ & $\mathrm{x}$ & $\mathrm{x}$ \\
\hline
\end{tabular}

${ }^{a}$ Operation of first flights will be during test phase of Shuttle.

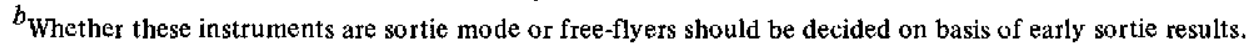

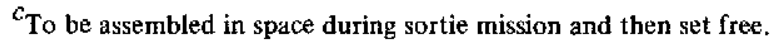


pursued in the 1970's to prepare the instrumental and astronomical foundations required for a Shuttleborne infrared program. The required scientific and technological advances can best be achieved by supporting a substantial effort in infrared astronomy by many individual research groups working on both ground-based and stratospheric infrared instruments.

This program will develop a pool of knowledgeable users for Shuttle-based astronomy as well as produce a variety of instrumentation and ideas that can be incorporated into the planning and construction of payloads for Shuttle flights in the 1980's. We consider the presentSR\& $T$ funding in this area to be totally inadequate for this purpose and deem it essential that the SR\&T budget be increased. The level of funding necessary to produce the desired balance between SR\&T and hardware represents a small fraction of the cost of a major orbiting payload, yet the SR\&T program will increase the scientific payoff of infrared astronomy in space by a very substantial factor.

\section{SUMMARY AND RECOMMENDATIONS}

Infrared space astronomy will begin as a substantial effort during the Shuttle era. The primary aim in this field will be to set up observatories in space, in particular, a large $\left(1-1 \frac{1 / 2-m}{)}\right)$ cryogenically cooled telescope and a very large (3-10-m) ambient-temperature telescope. These instruments are appropriate for Shuttle transportation because of their large size and weight and because of the rapidly advancing nature of infrared instrumentation, which requires frequent modification and improvement.

Infrared telescopes are especially sensitive to potential gaseous and particulate Shuttle contamination. It appears that the infrared requirements on contamination levels can be met with some specialized Apollo-type contamination control. Some specialized small instruments, for which contamination requirements are particularly severe, will have to be launched as free-flyers.

Much of the operation of these observatories, and most of the data analysis and decision making, will be carried out by a groundbased team of scientists and by a control computer on the Shuttle. The mission specialist will be utilized during initial setup and instrument mode changes and for overcoming unanticipated problems.

Space observatories offer a potential for utilizing infrared instrumentation far more sensitive and advanced than can be used in ground-based observatories. Realizing this potential requires a contin- 
uing and substantial research effort with ground-based and stratosphere techniques to maintain the flow of new technology.

Specific recommendations for infrared and submillimeter Shuttle astronomy follow.

1. We recommend initiation of design of a cryogenically cooled 1-m class telescope for sortie missions.

2. We recommend that the use of the Large Space Telescope for infrared astronomy be pursued.

3. We recommend that a vigorous program of infrared astronomy be pursued in the 1970's in order to prepare the instrumental and astronomical foundation required for the recommended Shuttle program.

4. We recommend the creation of a Shuttle contamination board composed of engineers and astronomers to establish standards for contamination based on the requirements of astronomy and to participate in reviews of Shuttle design.

5. We recommend that provision be made for small gimbalmounted payloads attached to the Shuttle bay.

6. We recommend that the payload specialists, control console be designed for easy inclusion of equipment unique to individual experiments. 


\section{6 \\ Optical and Ultraviolet Astronomy}

\section{S C I E N T I F I C O B J E C T I VES}

\section{A. Solar System}

Optical telescopes aboard the Shuttle, or carried into orbit by the Shuttle, can increase our knowledge of solar system bodies in several important ways. They can particularly provide for high-resolution imaging, for spectroscopy without the intervening atmosphere of the earth, and for photometry and polarimetry with high spatial resolution. Broadly, the objectives are to investigate the dynamics, composition, and structure of planetary atmospheres and surfaces, of satellites and asteroids, and of comets. The general goal is to improve our knowledge of the origin and evolution of the solar system.

Much of the imaging, spectroscopy, and photometry of solarsystem bodies has to be distributed over a period of time. Planets are dynamic bodies with continuously changing observable characteristics. Clouds, dust storms, polar hoods, and polar caps form and move on Mars. Unidentified dynamic modes in the atmosphere of Jupiter produce continuous changes in the belts and cloud patterns. Faint atmospheric markings and spectroscopically variable regions circulate around Venus. Changes and motions in the atmosphere of Saturn are at the very margin of earth-based detection, while those of Uranus are out of reach. Cometary spectra, which also change, are submerged in the earth's airglow.

Studies of these phenomena are intensively pursued with earthbased telescopes to the limits possible. Planetary flybys and orbiters permit short-term glimpses of local detail but do not provide planetwide synoptic coverage. Thus, Shuttle and Shuttle-launched optical telescopes can fill a major need in solar-system science, greatly exceeding the limits of present earth-based observation in synoptic programs of imaging and spectroscopy. The observations have to be made at regular intervals during apparitions, utilizing either the Large 
Space Telescope (LST) or a long-focal-length diffraction-limited telescope aboard the Shuttle. The same instruments can reveal detail in comets' heads and can provide superior spectra of both real and simulated comets.

We do not yet know whether the universe abounds in planets; it is possible that there are billions per galaxy and perhaps many that are closely earthlike. This is not only an exciting scientific question for astronomers and biologists, but it is also one of great potential philosophical impact. Although direct-image detection of planets around other stars remains unlikely with foreseeable methods, the $1-\mathrm{m} f / 30$ diffraction-limited Shuttle telescope and the LST provide, in principle, an opportunity to push the precision of stellar radial velocity variations and stellar proper-motion fluctuations into the range needed to explore this question more seriously than heretofore possible.

\section{B. Stars and Stellar Systems}

Three satellites, OAO-A2, OAO-C, and ESRO-TD1, have now contributed to the investigation of stellar ultraviolet radiation, and a fourth one, IUE, is planned for operation in the 1977-1980 period.

The wealth of information provided by these satellites will allow astronomers to complete the first major step into the field of ultraviolet astronomy. Classification criteria will be better known, discrepancies between observations and model atmosphere predictions will be better identified, and many intrinsic properties of stars (rotation, luminosity, abundances, mass ejection) will have been analyzed for the brightest objects.

Although unpredictable developments are likely to come in this decade, several major objectives can readily be spelled out for the 1980 's. In some cases, these require the LST and a 1-m diffractionlimited Shuttle telescope; but a $0.5-\mathrm{m}$ photometric telescope will sometimes suffice, and ultraviolet survey cameras will play important roles. Emphasis will be on the study of larger samples (thus on fainter objects), on higher photometric and spectrophotometric accuracies, and on high time-resolution studies of light curves. A reference sample of a few hundred stars will have to be observed as accurately as possible at low, medium, and high spectral resolution in order to establish fully a set of standard stars and to investigate more thoroughly the dependence of ultraviolet classification criteria on physical parameters by comparisons with model atmosphere predictions. 
Photometric observations will have to be extended to larger samples, such as globular and open clusters, where the sensitivity of ultraviolet colors to stellar properties is likely to provide important clues to the understanding of stellar evolution. Such data will also improve the galactic distance scale through better photometric parallaxes. Among other developments, one can identify systematic studies at medium and high spectroscopic resolution for classification, for a mass-loss search in order to study driving mechanisms, for a search of stellar chromospheres in late-type stars, and for the abundance analysis of normal and abnormal stars in order to improve the understanding of the evolution of the galaxy.

The good seeing and the absence of scintillation that characterize space observations can be taken advantage of to investigate variable stars with high time resolution and high photometric accuracy. In addition to resolvable binaries and spectroscopic binaries, these include intrinsic variables, such as RR Lyrae stars, flare stars, and Cepheids.

\section{Interstellar Matter}

Though it has long been known that the space between stars contains gaseous and particulate matter, the variety and complexity of this material is only now becoming apparent. Some dozens of molecules are now known to exist, many of them polyatomic, and there is no reason to think that the present inventory is complete. Ultraviolet extinction measurements indicate interstellar dust characteristics quite different from those suggested by observations at visual wavelengths. Furthermore, several different constituents are probably present. This dust is not only of interest in itself but also in its interaction with the gaseous component of the medium, since it appears that the production of at least some molecules requires the presence of dust either as a shield from the interstellar ultraviolet radiation field or as the site of formation. The mechanisms involved cannot be established until the grain composition is better known, and this in turn will probably require an understanding of how the grains are formed. The LST and the 1-m diffractionlimited Shuttle telescope will be the most valuable for pursuing these studies in the 1980's.

Data now available indicate the general features of interstellar extinction in the ultraviolet-its variability from place to place and the prominent $\lambda 2200$ feature. However, these data are as yet available only at low spectral resolution from a small number of nearby 
stars. Furthermore, few, if any, reliable ultraviolet polarization observations exist, which would be of special interest at and near the $\lambda 2200$ feature. Extinction and polarization measurements for large numbers and types of stars in a variety of geometrics with respect to the dust grains, as well as ultraviolet observations of reflection nebulae, would make available important evidence concerning the composition and characteristics of the grains. With high spectral resolution, faint extinction features might be found that would be of great use in testing various grain models. Because of the large farultraviolet extinction, photographs with a Shuttle survey camera in this spectral region might delineate dust clouds better than is now possible.

Ultraviolet observations of Lyman- $\alpha$ absorption have an important advantage over $21-\mathrm{cm}$-wavelength radio observations in mapping the local distribution of hydrogen, in that the geometry of the former is much better known than that of the latter. Several astrophysically important elements have resonance lines in the ultraviolet for which observations provide better determinations of their abundances than heretofore possible. Studies of the relation between gas and dust grains can be made by detailed elemental abundance determinations and grain distributions in neutral hydrogen regions.

\section{Emission Nebulosities}

The existence of emission nebulosities formed from condensations in the interstellar medium near hot stars provides unusual opportunity for study of several important areas of astrophysics. These nebulosities are formed from residual gas after the formation of short-lived, massive stars that are extremely bright in the ultraviolet spectral region. The residual gas is partially photoionized by this ultraviolet radiation. The imbalance of gas pressures leads to a general expansion and evolution of the nebulosity, which provides a laboratory for study of plasmas under well-defined conditions but on a scale not possible on the earth. Since the phenomenon only occurs near recently formed stars, we expect this study to yield information about the actual processes of star formation itself. Moreover, the ionized gas is a highly efficient converter of stellar continuum energy to atomic line emission, which allows the study of not only the conditions of temperature and density within the gas but also of its elemental abundances. Not only is this method of abundance determination easier and more reliable than others, but it can be applied to other galaxies and to regions within them. These energetic sources 
can best be studied at ultraviolet wavelengths accessible from above the atmosphere and at higher spatial resolutions than possible with grouñu-based telescopes.

\section{E. Galactic Nuclei and Quasars}

Violent processes are taking place in the nuclei of many giant galaxies, especially radio and Seyfert galaxies. Although such a galaxy may be a hundred thousand light-years in diameter, a substantial fraction of its entire luminosity comes from a tiny core, in some cases perhaps only a few tens of light years in size. The output of energy may be billions of times that of the sun.

Ordinary large galaxies, such as our own or M31, display what may be much the same kind of process, but at only a thousandth or even a millionth of the rate.

If the red shifts of quasars arise from velocities of recession in an expanding universe, they are the most distant objects that we know and their intrinsic luminosities range up to a hundred times those of the most luminous Seyfert or radio galaxies. But the energy output of every quasar appears to vary, in some cases as rapidly as days or even hours, requiring them to be as small as the solar system. The problem of extracting $10^{45} \mathrm{ergs} / \mathrm{sec}$ from so small a volume of space is so severe that some astrophysicists have proposed a nonrecessionvelocity origin for much of the apparent red shift, allowing quasars to be relatively nearby and perhaps a thousandfold less luminous.

Space observations are essential to solving the riddles posed by galactic nuclei and quasars. The LST, a 1-m diffraction-limited Shuttle telescope, an ultraviolet survey camera on the Shuttle, and a very large light collector can each contribute in important ways to extending wavelength coverage, measuring the red shifts of fainter objects, resolving diameters and structures, and discovering fainter quasars. Only by learning the physical processes in such objects can we be comfortable with our understanding of basic physical laws. Only by becoming confident about their true remoteness in the universe can we use them as the ultimate probes mentioned in Section I.H.

\section{F. Intergalactic Matter}

The detection and measurement of the intergalactic medium may be one of the frontiers of astronomical research in the 1980's. Current measurements suggest that the mean density of intergalactic matter is very small. However, there is a possibility that the intergalactic me- 
dium may be nonuniform and that there may be intergalactic clouds. Detection of such clouds, if they exist, could be accomplished by ultraviolet spectroscopy of quasars, provided they are as distant as their red shifts imply. Both the 1-m diffraction-limited Shuttle telescope and the LST can contribute significant data.

Alternatively, ultraviolet spectroscopy of the brightest blue stars in external galaxies may give definitive results. For these stars, a very-large-aperture telescope (like the Very Large Light Collector described later in this report) with fairly good spatial resolution $(\simeq 0.3 \mathrm{sec}$ of $\mathrm{arc})$ will be required.

\section{G. Extragalactic Research}

An important scientific objective of the 1980's will be high spatial resolution studies of distant galaxies. We are interested in whether distant galaxies (red shift $>10$ percent) are similar to the nearby galaxies. At a red shift of 10 percent (distance of $6 \times 10^{8} \mathrm{pc}$ if the

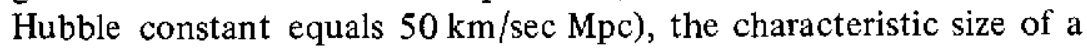
large spiral galaxy like M31 $\left(3 \times 10^{4} \mathrm{pc}\right)$ subtends an angular size of about $10 \mathrm{sec}$. An angular resolution of $0.1 \mathrm{sec}$ of arc or less is required to make detailed comparison of the structure and photometric profiles of these distant galaxies with the nearby galaxies. The distant ones require the LST, while the nearby ones require the field coverage of the deep-sky survey camera on the Shuttle.

In addition, it will be very interesting to compare the stellar content of nearby galaxies with our galaxy. At the present time, very little is known about stellar content beyond the Magellenic Clouds. The color-magnitude diagrams of star clusters in external galaxies will be important in establishing how similar the stars in different galaxies are. Besides its intrinsic interest, this knowledge will help to extend our stellar distance indicators to sufficiently large distances that local deviations from a smooth cosmic expansion can be investigated and that a better mean value for ultraviolet energy distributions of galaxies, including ellipticals, generally show a minimum at about $\lambda 2400$, followed by a rapid rise toward shorter wavelengths, which is too steep to be accounted for by any combination of early-type stars. This rise may be due to light scattered by dust and may therefore be useful in studying interstellar dust in other galaxies, along with its implication for infrared measurements, for computing the "K terms" of red-shifted galaxies, and for the interpretation of sky-brightness observations. 


\section{H. Cosmology}

Optical observations that relate to the origin and large-scale evolution of the universe have required the largest earth-based telescopes working to their very limits. By providing the possibility of putting major telescopes in orbit, the Shuttle opens the exciting opportunity of reaching deeper into the realm of galaxies, galaxy clusters, and quasars to seek better answers to the scientifically and philosophically challenging questions of cosmology.

in particuiar, one must search for distant ciusters of gaiaxies with a $1 \cdot \mathrm{m}$ deep-sky survey telescope reaching the 25 th magnitude in selected sky samples, measure red shifts and magnitudes of cluster members by spectrophotometry with a large light collector, and measure luminosity profiles of cluster members with a diffraction-limited LST. All of these can be expected to result in significant advances, and the last is certain to provide a major breakthrough in the determination of the deceleration parameter, based on plotting profile diameters against red shifts. The deceleration parameter distinguishes an infinite universe from a "closed" one, and that, together with the Hubble constant, specifies the age of the universe and the mean density of matter within it. The red shift-diameter relation is not so sensitive as the red shift-magnitude relation to corrections for evolutionary changes associated with the "look-back time."

One-meter Shuttleborne telescopes, as well as the LST can also aid the optical identification of radio galaxies and quasars, which may prove to be our ultimate probes of the geometry and evolution of the universe.

\section{I. CA N D I D A T E SH U T T LE-LA UN CHED I N S T R U M E N T S}

\section{A. The Large Space Telescope}

The Large Space Telescope (LST) is identified as by far the most important project for optical and ultraviolet astronomy in the first decade of operation of the Space Shuttle. It is uniquely capable of realizing most of the potential for imaging space astronomy and would represent an enormous step beyond the capabilities of possible ground-based telescopes. The next levels of astronomical and astrophysical research require this instrument.

To understand the potential of the LST, one must recognize the basic limitations imposed by the earth's atmosphere on ground-based 
observations. The three principal limitations arise from atmospheric opacity, atmospheric irregularities, and emission or scattering of light from the night sky.

The earth's atmosphere is partially obscuring at all wavelengths, but at some wavelengths it is so opaque as to block any penetration to the earth's surface. In the region of optical and ultraviolet astronomy, only a small fraction of the full wavelength range penetrates the atmosphere. Full wavelength coverage can be obtained by observation from satellite and rocket altitudes; this potential has begun to be exploited with small telescopes during the first decade of space astronomy but must now become a resource normally and continuously available to astronomy.

Small-scale inhomogeneities in the index of refraction of the earth's atmosphere lead to random deviations of the apparent direction of a star. To the eye, this phenomenon appears as the twinkling of stars, while a small-telescope observer sees an image that moves as the atmosphere appears to "boil." A large telescope gathers light passing through many regions of inhomogeneity and averages them out to form a stationary but large image. These phenomena are all familiar to the optical observer and are collectively known as "seeing," conventionally measured by the apparent diameter of a stellar image (the seeing disk). Seeing varies with time at any one observatory site, and its average value is different at different sites. The most frequent seeing disk with the $5-\mathrm{m}$ telescope at Palomar Mountain is about $2.5 \mathrm{sec}$ of arc in diameter, while that reported with smaller telescopes in the Andes may be as small as $1 \mathrm{sec}$ of arc.

The inherent imaging capabilities of a precisely made large telescope are remarkably much better than this limit set by atmospheric seeing. The diameter $(d)$ of a visible-light image, limited only by diffraction of light as it passes the primary aperture of diameter $(D)$ in meters, is given approximately by $d=0.15 / D \mathrm{sec}$ of arc; $d$ is also proportional to the wavelength of observation. This means that a diffraction-limited 3-m telescope operating in space, free from atmospheric seeing, at $5000 \AA$ can give images of $1 / 20 \mathrm{sec}$ of arc, while at $2500 \AA$ the images are only $1 / 40 \mathrm{sec}$ of arc in diameter.

The advantages of improved spatial resolution from a large diffraction-limited space telescope are twofold. The first is, of course, the ability to resolve structures in astronomical sources that are some 20-50 times smaller than can be seen from the ground. Since the gain is made in two dimensions, the effect is to give some 400 to 2500 times more information elements per area of the sky. Some 
feeling for so enormous an improvement can be realized by comparing the naked-eye view of the moon with that obtained by looking through a good 30-cm telescope. Most astronomical sources will show new structure down to the diffraction limit, while many star fields are so crowded that this improved resolution is required if we are to be able to study any but their brightest stars.

When one tries to detect faint starlike objects, the most important source of noise in the signal comes from the background night-sky brightness superimposed on the star image and its immediate vicinity. A darker sky permits the detection of fainter objects. Likewise, inherently smaller images mean less background light per image area and therefore again a fainter limiting threshold signal. Assuming a four times fainter sky from orbit and 50 times better images, a 3-m LST could detect objects nearly 100 times fainter than could the largest operational telescopes on earth. In turn, this means seeing nearly all kinds of stars out to ten times the present limiting distance, hence to bringing 1000 times the volume of space within view. Although these improvements may not be quite so great when very large telescopes are completed at superb sites, the gains will always remain extraordinarily great and attainable only by putting major telescopes in space.

The advantages of a large, high-spatial-resolution LST are many. A whole new regime of astronomical problems can be attacked at very low light levels, and much current threshold work can be carried out with improved accuracy. In addition, it will allow the spatial resolution of known sources and the discovery of new sources. It will be a powerful complement to all other disciplines of space astronomy, from infrared through gamma rays.

To realize these remarkable potentials of the LST, certain conditions are required:

1. It must have as large an aperture as possible, compatible with constraints of cost of manufacture and launch by the Shuttle.

2. It must be of as high optical performance as possible, compatible with total system costs.

3. It must be a true space observatory; that is, it must be a long-lived facility capable of refurbishment and repair over a span of many years.

4. The auxiliary instrumentation should reflect the needs and wishes of a diverse user community, compatible with efficient instrument design and operation. The program should allow the periodic 
re-evaluation of the instruments then being flown and possible selection of new instruments for flight.

5. The ultraviolet wavelength range should extend far down, if possible, to the Lyman continuum cutoff around $900 \AA$.

\section{B. Diffraction-Limited Telescope}

As prime complement to the LST program, we recommend a medium-sized Shuttle telescope for testing experimental instruments and detectors, for performing tasks requiring manned access to instruments, for conducting varied observational programs of short duration, for photographic and electronographic image recording, and for covering sky areas up to 30 times larger than can be imaged by the LST. These functions would be served by a diffraction-limited $f / 30$ Cassegrain telescope of about $1-\mathrm{m}$ aperture. The opportunity for utilizing photography and electronography arises because such materials will tolerate a space environment for the duration of a Shuttle flight (say, one month) but not for the much longer periods that would be required by the LST. A Shuttle telescope is therefore not limited in function and field coverage by the necessity for remote data readout. A sky field of up to $30 \mathrm{~min}$ of arc can be made optically available. Both wide-field electronography and smaller-field digital detectors can be expected to reach a threshold of about 27 th magnitude and thus for many purposes to exceed the performance of the best ground-based telescopes.

The proposed $1-\mathrm{m} f / 30$ Cassegrain design is suitable for mounting at the far end of the pallet, where it helps to put the center of gravity of the Shuttle within required limits. It can be equipped to perform two to three types of observation with command and monitoring either from the ground or from the Spacelab. If, for example, widefield imaging is provided by a film camera (or by the electronographic equivalent) mounted at the normal Cassegrain focus, it would still be easy to do spectroscopy or photometry of individual objects by inserting small diagonal mirrors ahead of the focal plane to feed a spectrograph and a photometer mounted at the side of the tube. In the same way, synoptic planetary image recording can be included on every mission with little or no conflict with other programs, particularly since such work could utilize bright daytime portions of the orbit.

If a coude configuration is adopted in place of a conventional Cassegrain, the optical focal plane can be made directly available to manned access inside the Spacelab. In general, this mode of opera- 
tion currently appears to offer more disadvantages than advantages, but it is certainly an option that could be developed if sufficient need arises.

\section{Small General-Purpose Telescope}

A useful astronomical instrument for the Shuttle sortie mode appears to be a modest, general-purpose uv telescope. This instrument could be an $f / 15$ Cassegrain of about $50-\mathrm{cm}$ aperture able to accept a variety of auxiliary instruments permitting photometric, spectrophotometric, and polarization measurements. Just as ground-based photometry has continued to be an important observational technique even after many decades, the same situation will certainly prevail in the uv. Furthermore, no other uv photometric instrument with these capabilities is currently envisaged.

The Orbiting Astronomical Observatory, OAO-2, barely began the study of a large number of interesting objects, e.g., magnetic variables, Cepheids, and close binaries, for which uv light curves are proving to be exceedingly interesting; surface photometry of globular clusters and galaxies; and color-magnitude diagrams of stars in open clusters. It should also be possible to utilize the superior guidance of the proposed instrument to observe nuclei of Seyfert galaxies and the brightest QSO's. A particularly interesting application of this instrument might be to observe, simultaneously with high-energy detectors, various X-ray objects or suspected sources. OAO showed the usefulness of having even small telescopes in orbit able to study transient objects like comets, novae, and supernovae. The $50-\mathrm{cm}$ telescope, although not always in orbit, would be available for launch rather quickly when such opportunities arose (this is an important feature of the sortie mode but one that probably diminishes with increasing payload complexity). In addition, a modest uv photometer is needed to utilize fully the capabilities of the LST. For example, it will enable absolute uv calibrations, typically made on very bright objects, to be extended to stars sufficiently faint for the LST to observe without overloading its detectors.

A typical instrument for this telescope might consist of a simple low-resolution uv spectrograph feeding an intensifier coupled to a relatively low-spatial-resolution area detector, e.g., of the silicondiode-array type. Thus simultaneous multiband photometric observations of the program object, as well as of the sky background, could be made with considerable advantages in observing efficiency and accuracy. With such a system intermediate-band photometry of un- 
reddened early-type stars as faint as 15 th magnitude could be done in a few minutes during the dark portion of the orbit. Bright stars could probably be observed during the sunlit portion.

In its simplest mode of operation, the telescope mount could be clamped on a small section of pallet, using Shuttle attitude and orbital data as the reference from which to point the telescope to within $0.5^{\circ}$; a simple bore-sighted star tracker could provide $1 \mathrm{~min}$ of arc pointing. The pointing stability would be whatever the Shuttle provided, preferably 1 or $2 \mathrm{~min}$ of arc. Alternatively, a simple openloop gyro system in the mount could provide $\sim 1 \mathrm{~min}$ of arc stability over some minutes of time. A simple TV system would give an observer on the ground occasional access to the image plane and would enable him to check pointing and star acquisition.

Observing programs could be transmitted to the Shuttle a few times each day for storage and later execution from a small on-board computer; data could be stored for transmission to a ground station at a convenient time. Data rates would be modest, only occasionally exceeding a few kilobits per second.

To observe the faintest objects or to work in crowded fields, the instrument could be mounted on a stabilized platform giving pointing accuracy and stability of a few seconds of arc. In either case, a total system is planned to weigh no more than $150-200 \mathrm{~kg}$, to use no more than about $50 \mathrm{~W}$ of Shuttle power, and to have only loose thermal control requirements for maintaining image quality. It would need no Spacelab facilities (but would need a few feet of electronic rack space somewhere in the Shuttle cabin) and would require astronaut attention only on special occasions. The Shuttle-payload interface would be simple, much like that on an Aerobee rocket, since the Shuttle would provide only power, telemetry, attitude data, and mechanical reference planes. Since this should be a relatively inexpensive instrument, two should be available for quick turnaround and to take advantage of every possible flight opportunity.

Such a simple but versatile telescope, occupying so little space and payload might well become the most widely used astronomical instrument of the Shuttle sortie mode, flying as often as a dozen times a year as a noninterfering accompaniment of a wide variety of primary missions.

\section{Very-Wide-Field Survey Camera}

Nearly all observations with rockets and satellites have been devoted to studies of individual stars and have given little information about 
the large-scale distribution of the stellar clouds of the Milky Way. Detection of interstellar matter (diffuse and emission nebulae) under the most favorable condition of almost no atmospheric sky background has not yet been undertaken.

A general optical study of the Milky Way needs a very wide field, partly to guard against the photometric difficulties of the conventional mosaic overlapping and partly to reach the extragalactic sky background on both sides of the Milky Way. Also, any large-scale evaluation of the distribution of galactic light is disturbed by interplanetary scattering from zodiacal light and gegenschein, which have angular sizes of the same order; it is thus essential to record galactic light and interplanetary scattering on the same field under the same conditions.

The instrument required by these constraints must have a very wide field $\left(\geq 60^{\circ}\right)$, be free of vignetting, and operate at a high focal ratio $(f / 1$ to $f / 2)$. Pointing accuracy of a few degrees is sufficient; guidance of $3 \mathrm{~min}$ of arc is adequate, but $1 \mathrm{~min}$ of arc would be better. Exposure times would range from a few seconds up to $30 \mathrm{~min}$ when working through narrow interference filters.

This instrument would preferably be operated by astronauts in a semiautomatic airlock mode, making it possible to get the camera back inside to recover and change film magazines, to change filters, and to adjust optical parts, for example. Alternatively, this verywide-field camera would easily fit a hitchhiker mode. In either case, it would be relatively small $\left(0.4 \times 0.4 \times 1.2 \mathrm{~m}^{3}\right)$ and lightweight (about $50 \mathrm{~kg}$ ).

\section{E. Very Large Light Collector}

Astrophysical study of any class of object usually follows a sequential pattern involving an increasing sophistication of analyzers and use of the flux at progressively higher resolutions. The present program of NASA for construction of an LST meets the needs for detection and initial analysis of the faintest cosmological sources but will not enable the researcher to study them in great detail. These first steps are essential, but we should not forgo plans for the next generation of telescopes.

In particular, there will always exist a class of sources that we can detect with our most powerful telescopes such as the LST but that have flux levels so low and consequent photon arrival rates so slow that it is impossible to perform accurate photometric or highspectral-resolution analysis. For such sources, we require a telescope 
of dramatically larger aperture but relatively modest optical quality. This need could be well met by the construction in space of a multiple-mirror telescope of some 10-m aperture or larger. Since the costs associated with a telescope of this type would increase only slowly with size, the total cost should not be inordinate.

Although this is not the place for a detailed design of such a telescope, some appropriate general properties can be inferred from its necessary constraints and goals. The individual optical elements should be large enough to permit individual diffraction-limited performance better than $0.1 \mathrm{sec}$ of arc but small enough to be easily fabricated and readily handled in space. If the array were operated as a single large segmented prime-focus mirror, each mirror element would depart only slightly from a flat surface and would accordingly be relatively quick and economical for an optician to produce. Almost continuous high-precision alignment of each mirror would probably prove necessary, requiring techniques of active optics. The frame of the array would presumably require prefabricated snap fittings permitting assembly in orbit.

Guidance, control, and instrumentation might be copied with as few modifications as possible from those developed for the LST, at considerable savings in development cost.

Two Shuttle flights should suffice to carry all parts of the system into orbit and to assemble it. Alternatively, the system could be carried up piecemeal over several years on other dedicated flights, as extra payload to be left in orbit; it could even be partly assembled and used with only a few elements while awaiting gradual completion. This more leisurely mode of construction might lead to appreciable savings, while permitting important astronomy research to be done from the very beginning.

Finally, the lifetime of the array should be indefinite. It is unlikely that the mirrors would deteriorate significantly, but, if so, the removal of a few at a time for resurfacing on the ground and later replacing would have little effect on the efficiency of the system.

The potential advantages inherent in this approach are so great as to warrant serious detailed studies to begin in the relatively near future.

\section{F. Other Instruments}

1. ALL-SKY ULTRAVIOLET SURVEYS

All-sky surveys have four major functions: to provide finding and reference data on the many objects that become of interest as astron- 
omers pursue their varied researches; to provide source data for statistical studies and synoptic data for features such as absorption clouds seen clearly only when examined over a wide field; to provide a historical record for objects that prove variable or transient; to permit the discovery of unusual objects and unexpected phenomena. Good surveys retain utility over many decades; they also serve a much larger number of users than is the case with most research. Their cost per data bit is very low, yet the value of the discoveries and studies made with their aid may be equal to that of the most highly specific projects.

Ultraviolet surveys using a meter-class all-reflecting Schmidt, reaching $m=19$ or 20 photographically (fainter, if electronic cameras are available), should be centered at wavelengths on the peak of the interstellar reddening curve $(2200 \AA)$ and at its far-uv minimum around $1500 \AA$. A low-resolution (about $100 \AA$ ) objective prism survey with the same instrument would reach about 15 th magnitude, bringing out objects with anomalous uv spectra, including bright radio-quiet QSO's. A higher-resolution survey (about I A) would reach $m=10$, with spectra permitting detailed astrophysical studies of tens of thousands of stars.

The various catalogs would augment other sources of objects for more detailed study, particularly with the LST. They would permit improved first-order selection of candidate identifications for optical counterparts of radio, x-ray, and gamma-ray sources. They would be particularly valuable for working out problems related to galactic structure and for correction of extragalactic observations as distorted by extinction in our galaxy. Intergalactic absorption and emissions may be detectable by such surveys. While there is as yet no reason to expect the discovery of new classes of objects with enormously enhanced uv radiation, nevertheless we will not be sure until an exhaustive search has been made; even a very few could be important.

These typical applications indicate the properties of the instrument needed for such work. To cover the necessary wide spectral range, it would have to be an all-reflecting Schmidt. If possible the field should be external and flat to permit use of large rolls of film or of electronic cameras. The aperture must be sufficient $(0.5$ to $1 \mathrm{~m})$ to reach faint objects in a reasonable time (the uv sky is several magnitudes darker than the visual sky, permitting deeper penetration for a given $f /$ ratio; but the typical source brightness and number of photons are lower in the visual, requiring longer exposures to pick up faint objects). A resolution of $1 \mathrm{sec}$ of arc is desirable. The field must 
be large enough to permit rapid coverage of the sky, thus at least $5^{\circ}$ in diameter.

These all-sky surveys are particularly well suited to extended 28-day Shuttle flights (about thirty-two 15-min dark-sky exposures per day; 900 per flight; 1800 required to cover the entire sky). Even using film, two such missions with the sun in opposite hemispheres of the sky would provide complete coverage for each program, thus requiring a total of eight flight opportunities during the 1980's. If suitable electronographic cameras become available, each survey would need less than half of the number of days, while reaching more than a magnitude fainter; the programs could thus be accomplished on only four Shuttle flights with substantial cost savings even after including the expense of developing the electronographic camera (which would also have important uses in other parts of the space program). A folded Schmidt with $0.75-\mathrm{m}$ aperture, able to accomplish these programs, would require about $2 \times 3 \times 4 \mathrm{~m}^{3}$ of space and weigh about $2500 \mathrm{lb}$.

\section{DEEP-SKY-SURVEY 1-METER TELESCOPE}

Sky fields intermediate in size between those provided by a generalpurpose $f / 30$ Cassegrain telescope and by a wide-field Schmidt survey camera would be very useful for some important search and survey problems. Such a system would be particularly powerful for observations of details of diffuse nebulae and $\mathrm{H}$ il regions in our galaxy, of the structure and stellar populations of the nearest galaxies, of intergalactic matter and bridges between systems of galaxies, of very distant clusters of galaxies (red shifts beyond $z=0.5$ ) for cosmological studies, and of selected sky areas to search for optical counterparts of radio and $x$-ray sources beyond the threshold of the optical and uv all-sky surveys.

These goals would be served well by an existing RitcheyChrétien-Bowen design that provides excellent imaging at $f / 7$ over a $3^{\circ}$ field. With a $1-\mathrm{m}$ aperture, it can be expected to have a threshold about 3 magnitudes fainter than the all-sky survey camera described above. This optical system, as a package, can be substituted for the $f / 30$ Cassegrain system into the same mounting structure, and it has simpler pointing and stabilization requirements. Sky-limited exposures can be approached in some cases with unaided photography and can be easily reached at all optical wavelengths with electronography. For photographic work on very faint diffuse sources or for the widest-field electronographic studies, a reducing camera option may be desirable. 


\section{SOLAR VARIATION}

The total emission of energy by the sun is an important parameter in meteorology and climatology. The solar luminosity is normally assumed not to vary significantly-hence the term "solar constant" for the flux received at the standard distance of $1 \mathrm{AU}$. However, observational evidence for constancy is only at about the 1 percent level, largely because of the difficulty in removing atmospheric effects from the observations, especially in the uv part of the spectrum.

The Shuttle offers the possibility of very frequent observations over all the significant wavelength regions from 0.1 to $10 \mu \mathrm{m}$. To be most useful the photometric instrument should accept uniformly the entire disk of the sun, be very simple and light-weight, rugged, easy to calibrate, accurate to at least 0.01 percent, and inherently free of significant instrumental change or drift over a decade or more of use.

The entire instrument should be only a fraction of a meter in size, weighing less than $20 \mathrm{~kg}$, and requiring only a few watts of power. It could be operated by astronauts through an airlock or could be fastened somewhere inside the Shuttle bay for use once or twice for a few minutes during a mission.

\section{MICHELSON INTERFEROMETER}

Angular resolution beyond that of the LST $(\simeq 0.035 \mathrm{sec}$ of arc in the visible) will probably require interferometric techniques. As an example, a $30-\mathrm{m}$ Michelson interferometer is capable of measuring spatial frequencies as small as $0.0035 \mathrm{sec}$ of arc at visible wavelengths and $0.001 \mathrm{sec}$ of arc in the far uv. Such ultra-high resolution would provide powerful constraints on theories of QSO's and galactic nuclei. Very close binaries and many stellar diameters could be resolved. Even surface details of certain stars could be studied, particularly red giants, where large-scale convective cells are suspected on theoretical grounds and where there is evidence, in at least one case, for nonspherical shape.

However, severe technical requirements must be met before such an interferometer could be used for aperture synthesis. An rms guidance jitter of the order of 0.1 times the resolution $(0.0001 \mathrm{sec}$ of arc in the most severe case) is required to measure the phase and amplitude of the spatial frequencies.

Although the sortie mode makes it more difficult to achieve the guidance requirements, it does allow manned participation in erecting the interferometer in space and in learning how to operate it. Further discussion of this topic will require a feasibility study. 
TABLE 15 Payload Requirements

\begin{tabular}{|c|c|c|c|c|c|c|c|c|c|c|}
\hline & $\begin{array}{l}\text { Large Space } \\
\text { Telescope } \\
\text { Free-Flyer }\end{array}$ & $\begin{array}{l}\text { I-m Diffrac- } \\
\text { tion-Limited } \\
\text { Telescope }\end{array}$ & $\begin{array}{l}0.5 \cdot \mathrm{m} \text { General- } \\
\text { Purpose } \\
\text { Telescope }\end{array}$ & $\begin{array}{l}\text { Yery-Wide-Fiteld } \\
\text { Camera }\end{array}$ & $\begin{array}{l}N \text {-meter } \\
\text { Light } \\
\text { Collector }\end{array}$ & $\begin{array}{l}\text { Uv Survey } \\
\text { D.75-m Telescope }\end{array}$ & $\begin{array}{l}\text { Deep-Sky } \\
\text { Survey } \\
\text { l } \mathrm{m} \\
\text { Telescope }\end{array}$ & $\begin{array}{l}\text { Michelsan } \\
\text { Interferometer }\end{array}$ & $\begin{array}{l}\text { Solar Constent } \\
\text { Variation }\end{array}$ & $\begin{array}{l}\text { 1-m-Wide- } \\
\text { Field Telescope }\end{array}$ \\
\hline $\begin{array}{l}\text { A. Man A rsendance } \\
\text { Payload Specialist } \\
\text { Astronames }\end{array}$ & $\begin{array}{l}<24 \mathrm{~h} \\
\text { For check-out } \\
\text { Optional }\end{array}$ & $\begin{array}{l}\text { 0-SO\% } \\
\text { Occasional } \\
\text { Optional }\end{array}$ & $\begin{array}{l}\text { For mulfurction } \\
\text { only } \\
\text { Rase } \\
\text { None }\end{array}$ & $\begin{array}{l}\text { 0-20\% } \\
\text { Occassional } \\
\text { Optionat }\end{array}$ & $\begin{array}{l}\text { FBD } \\
\text { Mirror Assembly } \\
\text { in space }\end{array}$ & $\begin{array}{l}\text { Rare } \\
\text { Check-out } \\
\text { None }\end{array}$ & $\begin{array}{l}\text { Rare } \\
\text { Check-our } \\
\text { None }\end{array}$ & $\begin{array}{l}\text { Desirzble } \\
\text { Desirable } \\
\text { Desirable }\end{array}$ & $\begin{array}{l}\text { None } \\
\text { None } \\
\text { None }\end{array}$ & $\begin{array}{l}\text { Rare } \\
\text { Check-out service } \\
\text { Optional }\end{array}$ \\
\hline $\begin{array}{l}\text { B. f. Pointing } \\
\text { lnstrument } \\
\text { Orbiter } \\
\text { 2. Stabilization }\end{array}$ & $\begin{array}{l}\text { N.A. } \\
\text { N.A. }\end{array}$ & $\begin{array}{l}\text { Few sec of arc } \\
\leq \pm 30 \text { deg }\end{array}$ & $\begin{array}{l}0.1-1 \text { min of are } \\
\leq \pm 30 \mathrm{deg}\end{array}$ & $\begin{array}{l}1-3 \mathrm{~min} \text { of arc } \\
\leq \pm 30 \mathrm{deg}\end{array}$ & N.A. & $\begin{array}{l}1-3 \text { min of arc } \\
\leq \pm 45 \text { deg }\end{array}$ & $\begin{array}{l}1-3 \mathrm{~min} \text { of arc } \\
\leq \pm 45 \mathrm{deg}\end{array}$ & $\begin{array}{l}\text { Few sec of arc } \\
\text { Few deg }\end{array}$ & $\begin{array}{l}0.1 \mathrm{deg} \\
\leq \pm 45 \mathrm{deg}\end{array}$ & $\begin{array}{l}1-3 \text { min of are } \\
\leq \pm 45 \mathrm{deg}\end{array}$ \\
\hline $\begin{array}{l}\text { Imase } \\
\text { Orbiter }\end{array}$ & $\begin{array}{l}\text { N.A. } \\
\text { N.A. }\end{array}$ & $\begin{array}{l}1 / 30 \mathrm{sec} \text { of arc } \\
0.1 \mathrm{deg}\end{array}$ & $\begin{array}{l}0.1-1 \text { min of arc } \\
0.1 \text { des }\end{array}$ & $\begin{array}{l}1-3 \min \text { of } a r c \\
0.1 \mathrm{deg}\end{array}$ & $\begin{array}{l}\text { N.A. } \\
\text { N.A. }\end{array}$ & $\begin{array}{l}0.1 \mathrm{gec} \text { of atc } \\
0.1 \mathrm{deg}\end{array}$ & $\begin{array}{l}0.1 \mathrm{sec} \text { of are } \\
0.1 \mathrm{deg}\end{array}$ & ${ }_{a}^{10^{-3}-10^{-4} \text { aec of are }}$ & $\begin{array}{l}0.1 \mathrm{deg} \\
0.1 \mathrm{deg}\end{array}$ & $\begin{array}{l}1 \mathrm{sec} \text { of } \mathrm{atc} \\
0.1 \mathrm{deg}\end{array}$ \\
\hline C. Contamitation & \multicolumn{10}{|c|}{ To be specified lates on the basis of a more detailed study } \\
\hline $\begin{array}{l}\text { D. Thermal Requirement } \\
\text { Temperature }\end{array}$ & $a$ & $e_{0-20}{ }^{\circ} \mathrm{C}$ & ${ }^{a} 0-20^{\circ} \mathrm{C}$ & $a_{0-20^{\circ} \mathrm{C}}$ & ${ }^{a} 0-20^{\circ} \mathrm{C}$ & $a_{0}-20^{\circ} \mathrm{C}$ & $a_{0-20^{\circ} \mathrm{C}}$ & $a$ & - None & ${ }^{a} 0-20{ }^{\circ} \mathrm{C}$ \\
\hline $\begin{array}{l}\text { E. Orbit Inclination } \\
\text { Orbit Altitude }\end{array}$ & $\begin{array}{l}0^{\circ} \text { Dexirable } \\
2 \mathrm{~B}^{\circ} \text { Acceptable } \\
500-800 \mathrm{l}: \mathrm{m}\end{array}$ & $\begin{array}{l}0^{\circ} \text { Desirable } \\
<35^{\circ} \text { Acceptable } \\
300-800 \mathrm{~km}\end{array}$ & $\begin{array}{l}0^{*} \text { Desirable } \\
<35^{\circ} \text { Acceptable } \\
250-800 \mathrm{~km}\end{array}$ & $\begin{array}{l}0^{\circ} \text { Desirable } \\
\angle 35^{\circ} \text { Acceplable } \\
300-800 \mathrm{~km}\end{array}$ & $\begin{array}{l}0^{\circ} \text { Desirable } \\
<35^{\circ} \text { Acccptable } \\
500-800 \mathrm{~km}\end{array}$ & $\begin{array}{l}0^{\circ} \text { Desirable } \\
<3 S^{*} \text { ACeeptable } \\
300-800 \mathrm{~km}\end{array}$ & $\begin{array}{l}0^{a} \text { Desirable } \\
<35^{\circ} \text { Acceptable } \\
300-800 \mathrm{~km}\end{array}$ & $\begin{array}{l}0^{\circ} \text { Desirable } \\
<35^{\circ} \text { Acceptable } \\
300-800 \mathrm{~km}\end{array}$ & & $\begin{array}{l}0^{\circ} \text { Desirable } \\
<35^{\circ} \text { Acceptable } \\
300-800 \mathrm{~km}\end{array}$ \\
\hline $\begin{array}{l}\text { F. Protection from } \\
\text { Charged Particles }\end{array}$ & N.A. & $\begin{array}{l}\text { Desirabie for } \\
\text { film }\end{array}$ & $\begin{array}{l}\text { Desirable for } \\
\text { film }\end{array}$ & $\begin{array}{l}\text { Desirable for } \\
\text { fitm }\end{array}$ & $\begin{array}{l}\text { Desirable for } \\
\text { film }\end{array}$ & $\begin{array}{l}\text { Desirable for } \\
\text { film }\end{array}$ & $\begin{array}{l}\text { Desiruble for } \\
\text { filłm }\end{array}$ & $a$ & None & $\begin{array}{l}\text { Desirable for } \\
\text { film }\end{array}$ \\
\hline $\begin{array}{l}\text { G. Weizht } \\
\text { Size } \\
\text { Power }\end{array}$ & $\begin{array}{l}22,000 \mathrm{ib} \\
32 \mathrm{~m} \\
\text { N.A. }\end{array}$ & $\begin{array}{l}9000 \mathrm{lb} \\
3 \mathrm{~m} \\
3 \mathrm{~kW}\end{array}$ & $\begin{array}{l}400 \mathrm{lb} \\
1 \mathrm{~m} \times 2 \mathrm{~m} \\
50 \mathrm{w}\end{array}$ & $\begin{array}{l}100 \mathrm{lb} \\
0.5 \mathrm{~m} \times 1 \mathrm{~m} \\
50 \mathrm{~W}\end{array}$ & $\begin{array}{l}a \\
\text { N.A. }\end{array}$ & $\begin{array}{l}2500 \mathrm{lb} \\
2.5 \mathrm{~m} \times 4 \mathrm{~m} \\
1 \mathrm{~kW}\end{array}$ & $\begin{array}{l}6000 \mathrm{lb} \\
2.5 \mathrm{~m} \times 4 \mathrm{~m} \\
1 \mathrm{kw}\end{array}$ & Up to $30 \mathrm{~m}$ & $\begin{array}{l}50 \mathrm{lb} \\
1 \mathrm{ft} \times 1 \mathrm{ft} \\
\text { Few watts }\end{array}$ & $\begin{array}{l}6000 \mathrm{Ib} \\
2.5 \mathrm{in} . \times 4 \mathrm{in} \\
1 \mathrm{~kW}\end{array}$ \\
\hline $\begin{array}{l}\text { H. Commands * } \\
\text { TM Bit Rare } \\
\text { Video Bit Rate }\end{array}$ & N.A. & $\begin{array}{l}20^{b} \\
2 \mathrm{kbits} / \mathrm{sec} \\
20-100 \mathrm{kbps}\end{array}$ & $\begin{array}{l}10^{b} \\
<2 \mathrm{kbits} / \mathrm{sec} \\
10-100 \mathrm{kbps}\end{array}$ & $\begin{array}{l}<10^{b} \\
<0.5 \mathrm{kbits} / \mathrm{sec} \\
\text { Optional }\end{array}$ & $\begin{array}{l}\text { N.A. } \\
\text { N.A. } \\
\text { N.A. }\end{array}$ & $\begin{array}{l}20^{b} \\
2 \text { kbps } \\
20-100 \text { kbps }\end{array}$ & $\begin{array}{l}20^{h} \\
2 \text { kbps } \\
20-100 \text { kbps }\end{array}$ & $a$ & $\begin{array}{l}5 \\
<0.5 \mathrm{kbps} \\
\text { Optional }\end{array}$ & $\begin{array}{l}20^{b} \\
2 \text { kbps } \\
20-100 \text { kbps }\end{array}$ \\
\hline $\begin{array}{l}\text { D. Detectors } \\
\text { Data Storage } \\
\text { Processing }\end{array}$ & $\begin{array}{l}\text { N.A. } \\
\text { N.A. } \\
\text { N.A. }\end{array}$ & $\begin{array}{l}\text { Film/Area } p / \mathrm{PM} \\
\text { Few Mbits } \\
\text { Optionsl } \\
\text { b }\end{array}$ & $\begin{array}{l}\text { Film/A rea D/PM } \\
\text { Few Mbits } \\
\text { Optional }^{b}\end{array}$ & $\begin{array}{l}\text { Film/Area } D \\
\text { Few Mbits } \\
\text { Optional }\end{array}$ & $\begin{array}{l}\text { N.A. } \\
\text { N.A. } \\
\text { N.A. }\end{array}$ & $\begin{array}{l}\text { Film/Area } D \\
\text { Few Mbits } \\
\text { Optional }\end{array}$ & $\begin{array}{l}\text { Film/Area D } \\
\text { Few Mbils } \\
\text { Optiona! }\end{array}$ & $\begin{array}{l}a \\
\text { Desirable } \\
b\end{array}$ & $\begin{array}{l}\text { Solid state } \\
\text { [00 kbits } \\
\text { Optional }\end{array}$ & $\begin{array}{l}\text { Fim/Area D } \\
\text { Few Mbits } \\
\text { Optional }\end{array}$ \\
\hline $\begin{array}{l}\text { K. Conluct with } \\
\text { ground }\end{array}$ & $\begin{array}{l}\text { During } \\
\text { check-put }\end{array}$ & $\begin{array}{l}\text { Access } \\
\text { at any time; } \\
\text { 5 } 100 \text { minjortit }\end{array}$ & $\begin{array}{l}\text { Access } \\
\text { al any time; } \\
\quad 530 \mathrm{~min} / \mathrm{arbit}\end{array}$ & Occasionai & 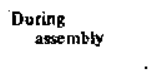 & $\begin{array}{l}\text { Access } \\
\text { it any time; } \\
5-60 \text { min/orbit }\end{array}$ & $\begin{array}{l}\text { Access at } \\
\text { any time; } \\
5-60 \text { min/orbst }\end{array}$ & Desirable & Optional & $\begin{array}{l}\text { Access at } \\
\text { any time: } \\
5-60 \text { min/orbit }\end{array}$ \\
\hline
\end{tabular}

\section{"To be delermined.}

$b_{\text {inlerasl }} 10$ inrtrualent possible. 


\section{TECHNICAL IMPACT ON SHUTTLE}

\section{A. The Roie of Man in Space-Shutite Ástronomy}

There can be four levels of manned interaction with optical-uv astronomical instruments carried to space by the Shuttle: active observation by the astronomer, operation of observing programs including film change, repair or maintenance of equipment including detector change, and assembly of instruments too large or too heavy for single-Shuttle flights. Each of these is considered in more detail, not only in the remainder of this section but also where relevant in the description of individual instruments. (See also Table 15.)

\section{ACTIVE OBSERVATIONS}

While a case can be made for active human monitoring of certain transient phonomena such as solar flares, we believe that manned interaction with the observations is unlikely to prove an important mode for nonsolar optical and uv astronomy.

An exception to this statement would arise if a coude telescope mounting were adopted, bringing the image into the Spacelab for shirtsleeve access. Possible advantages with this scheme include ease of construction and change of instruments at the focus and slightly reduced need for full automatic pointing and guidance. However, the drawbacks seem much more severe, including primarily the weight of the Spacelab, which would preclude having both a substantial telescope and an extended mission (only an expected 5000-lb real payload for a 28-day mission including a minimal Spacelab); for nearly all contemplated uses the advantage of extended observing time and of flying several major instruments overwhelm any simplifications that might be possible from continuous human access. The telescope mounting and guiding in any event must be of the quality required by the final imaging; TV monitoring of setting and focus are already routine-even at ground-based observatories; and the limited observing time available even with extended Shuttle missions strongly suggests that each flight be optimized for use of typically one of at most two or three instruments at the focus.

\section{OPERATION OF OBSERVING PROGRAMS FOR PALLET. MOUNTED OR FREE-FLYING INSTRUMENTS}

Some of the astronomy missions would be likely to profit from planned extravehicular activity (EVA) at the start of operations, to check out the telescope and its auxiliary instruments, and in photo- 
graphic programs to recover a test exposure for immediate on-board development.

Assuming the likely event that EVA activity will become relatively straightforward and routine, the planned changing of film canisters or reconfiguring of telescopes during missions (e.g., from photometric to spectroscopic operation or the change to a different kind of detector) may well be more easily, reliably, and cheaply done by man than by remotely operating devices.

\section{MAINTENANCE AND REPAIR}

Although the LST will be a free-flying observatory, operated from the ground, its success will depend on revisits for service, maintenance, and refurbishment by the Space Shuttle.

The present NASA program for the LST has adopted as guidelines that the major repairs and changes will be made following earth return by the Shuttle, with only limited on-orbit service. Because of the unavoidable delays expected between ground return and relaunch, we hope that the on-orbit work can be identified as the most desirable method of normal operation. Since this would mean both servicing flights and return flights, the Shuttle must have docking facilities that permit pressure-suited manned entry of the LST and suitable storage structures for launch and recovery. Servicing visits should be made possible on a shared mission basis, allowing relatively small costs to be incurred. Likewise, the launch and recovery structures should be such as to permit flight sharing.

It would seem wise to design other free-flyers and sortie-mode instruments for some degree of manned access so that simple malfunctions could be cleared up by EVA. In particular, for some experiments it may be important to carry one or more complete spare detector packages and microcircuit electronic cards covering all essential functions.

\section{ASSEMBLY}

Only one telescope in the optical-uv area is likely to profit from any degree of manned assembly in space, and it will require this mode. A very large optical array or segmented-mirror telescope might need the equivalent of two full Shuttle payloads to bring its elements up to orbit, with several man-weeks of EVA for assembly.

\section{B. Pointing and Stabilization}

The pointing and stabilization requirements of a $1-\mathrm{m}$ diffraction- 
limited telescope are as stringent as those of any astronomical instrument that is likely to be flown in the sortie mode. If the higher optical spatial frequencies are not to be severely attenuated, the reduction in amplitude at the limiting frequency due to guidance errors must be less than 50 percent. For a $1-\mathrm{m}$ telescope operating at $5000 \AA$, the resulting guidance error is less than $0.02 \mathrm{sec}$ of arc rms. Imagery at shorter wavelengths would decrease the value to $0.01 \mathrm{sec}$ of arc rms.

A stabilization of $0.01 \mathrm{sec}$ of arc rms in the frec-flying mode has been shown feasible in several studies, notably the phase A report of the LST for which the requirements are even more stringent. Furthermore, the OAO-C (Copernicus) telescope is achieving a guidance of the order of $0.02 \mathrm{sec}$ of arc rms, as did the Stratoscope II balloonborne telescope.

An intermediate level of guidance required by some telescopes is about $1 \mathrm{sec}$ of arc rms. Experience with telescopes mounted aboard aircraft indicates that this level of guidance can readily be obtained using a gyro as a sensor.

At the lower end of the requirements, the \pm 0.1 deg of arc basic Shuttle stabilization is adequate for many important purposes, particularly the general-purpose telescope described above.

The current pointing specifications of the Shuttle are $\pm 0.5 \mathrm{deg}$ of arc, which is adequate for wide-field cameras that rely on the basic $\pm 0.1 \mathrm{deg}$ of arc stabilization of the Shuttle. More accurate pointing specifications can readily be accomplished with the aid of boresighted star trackers. Search modes, either with the telescope or with the Shuttle as a whole, may be helpful.

\section{Contamination}

In the present circumstances, it is impossible to discuss contamination problems in any but the most superficial terms. The contaminants likely from the Shuttle and their outgassing rates are unknown; detector types and surfaces that will be used in the 1980's are uncertain; and possible shielding, packaging, and other design techniques are not yet determined. However, it is clear that photocathodes and optical surfaces must not be exposed to contaminants such as $\mathrm{H}_{2} \mathrm{O}, \mathrm{O}_{2}$, or organic volatiles. Any change of sensitivity, either by degradation in cathode sensitivity, by optical reflectivity, or by absorption by a cloud of material surrounding the spacecraft, should not be greater than a few percent in any 1 or $2 \AA$ interval over 
a 30-day mission. Larger changes might require an inordinate amount of recalibration or compromise the photometric accuracy of the observing program.

The effects of light scattered by particles in the line of sight must be held to such a level that the artificial background induced is small compared with the natural level.

\section{Thermal Requirements}

Because of the very long thermal time constant of the primary mirror (typically days), most recent thermal studies of diffraction-limited telescopes have assumed that the primary and secondary mirrors are kept at a fixed temperature $\left(\simeq 20^{\circ} \mathrm{C}\right)$ by means of active thermal control. Most other portions of the optical telescope assembly have passive thermal control. It would seem that the same basic thermal design would be satisfactory for the sortie mode.

In thermal designs for the free-flying mode, the scientific instrument package generally has a thermostated portion containing the relay optics and other devices that generate little heat. In addition, heat-generating cameras and other instruments are coupled to the outer portions to allow the heat to be radiated to space.

In the sortie mode, the scientific instrument package must radiate its heat to the inside of the Shuttle bay rather than to space. Provided that the temperature of the Shuttle bay is kept sufficiently cool, it seems plausible that the basic free-flyer design could be reasonably adapted to the sortie mode.

Before such a conclusion is made, however, a study should be carried out to establish in detail the problems of adapting a free-flyer thermal design to the sortie mode. In particular, the study should determine whether the $6-\mathrm{kW}(\simeq 21,500 \mathrm{Btu} / \mathrm{h})$ cooling available to the pallet is adequate. Generally the thermal problems of other optical and ultraviolet telescopes are less severe than for a diffractionlimited telescope.

\section{E. Orbits}

The orbit for $1-\mathrm{m}$ telescope operation must be in the $300-800 \mathrm{~km}$ range, to optimize minimum reasonable sky brightness and radiation environment. The most desirable orbit is equatorial (in order to avoid the South Atlantic Anomaly), although if it is not available the standard $28.5^{\circ}$ inclination is nearly as satisfactory as any intermediate value. 


\section{F. Payload Weight}

The total weight of a $1-\mathrm{m}$ class sortie-mode telescope with its mounting will be about $7000 \mathrm{lb}$ plus about $2000 \mathrm{lb}$ of control equipment in the Shuttle cabin or a Spacelab. These numbers are based on the recently constructed 36-in. airborne telescope and are about one half of those of a recent industrial study. If the entire Shuttle is used for coarse pointing, thereby limiting the necessary pitch angles of the telescope, then a bay section of full width and 2-m length would be required. $\dot{A} 4-\mathrm{m}$ length would be required if Shuttle coarse pointing is not available. Control rack space of about $100 \mathrm{ft}$ would be necessary. Power requirements during operation will be approximately $3 \mathrm{~kW}$. The maximum weight of a free-flying satellite presently contemplated is that of the LST, which is almost $22,000 \mathrm{lb}$.

\section{G. Detectors and Telemetry Requirements}

All the major proposed optical and uv-astronomy missions are being planned to operate primarily by ground command. In some cases, a backup Shuttle control option may be useful. The data rates needed for the ground-based command and control functions are conventional and generally low; availability of an on-board computer is desirable and would be essential if the tracking and data-relay satellite should not be continuously available. Likewise telemetry requirements for reporting of housekeeping data would be negligible.

Except for photographic missions, the requirements for transmission of scientific data to the earth will be relatively high. Photometric field studies might use area detectors containing at least $10^{4}$ elements, to be read out normally at intervals of about a minute, leading to maximum bit rates of perhaps $10^{4} / \mathrm{sec}$. However, occasional high-speed photometric problems would require target readout at millisecond rates, or $10^{7} \mathrm{bits} / \mathrm{sec}$.

Digital television readout tubes and electronographic cameras are two of the prime candidates foreseen today for optical image detectors aboard the Shuttle in the 1980's. Whether they completely replace unaided photography will depend on the availability of fields up to 20 or $25 \mathrm{~cm}$ in diameter. Both have the potential of high photometric accuracy.

Some images may have as many as $2 \times 10^{9}$ pixels per picture or about $20 \times 10^{9}$ bits of data per picture, assuming a pixel size of about $5 \mu \mathrm{m}$. Even if data transmission rates can be increased to the $10 \mathrm{Mbits} / \mathrm{sec}$ range, more than $30 \mathrm{~min}$ would be required to transmit 
a picture to earth. The total production of image bits during a Shuttle mission could exceed $10^{12}$. Electronography, if expanded to the field size desired, comes close to meeting the desired storage goal.

A modest reduction of storage requirements would be possible by onboard processing of images if the data are in digital form. In many images, for example, an overwhelming majority of the pixels may all have the same brightness value, namely that of the sky background, and the full information contained in the image can be put into a more compressed format than a listing of brightness values separately for every pixel. There may consequently be a tradeoff between storage capacity and processing capability. At least some digital capability will be required to handle nonimage data, but the amount will doubtless be small in comparison with image data.

\section{MISSION MODEL}

An observatory in space, just as one on the ground, is extremely versatile and capable of carrying out a wide variety of measurements of an exceedingly large number of objects. Such a facility, be it a small 0.5-m uv telescope or the LST, is in no way an "experiment" in the generally accepted sense of that term. Thus the scientific value of a uv telescope increases with time in operation, since the information gained increases with observing time; there are few, if any, "saturation effects." Indeed, many types of investigating, e.g., in planetary science, are greatly increased in value if continuums or repeated observations can be made. It is for these reasons that we place primary emphasis on free-flyers, not only the LST but also smaller payloads. Even where the sortie mode is advantageous, to maximize the scientific return of the relatively small number of astronomy sortiemode flights, presented in the mission model, these flights should be extended beyond 7 days as soon as possible. (See Table 16.) Any such extension immediately increases the mission efficiency in that the times required to prepare for operations after launch as well as to prepare for re-entry and landing will be about the same regardless of mission length. Of prime importance, however, is to increase the astronomy mission lengths to 30 days or longer as soon as possible.

Much interesting and valuable work in the uv and visual wavelength regions can be carried out with small instruments, requiring very simple payload-Shuttle interfaces, e.g., the $0.5-\mathrm{m}$ generalpurpose telescope, the very-wide-angle camera, the solar-constant in- 
TABLE 16 Optical and Ultraviolet Sortie-Mode Missions, $1981-1991^{a}$

Major Optical and uy Instruments

Total number of stellar sortie payloads

61 or $\simeq 6 / \mathrm{yr}$

Likely number of optical and uv payloads

$\simeq 20$ or $\simeq 2 / \mathrm{yr}$

If during 1981-1985 mission length is only 7

days and 5 days/mission are available

for observing, then get

or

$\simeq 10$ observing days/yr

$\simeq 75 \mathrm{~h}$ of dark time $/ \mathrm{yr}$

Small Optical and uv Instruments

Total number of stellar sortie flights

$\simeq 30$ or $3 / \mathrm{yr}$

Assuming one small instrument on half of these flights would have

$\simeq 15$ or $1.5 / \mathrm{yr}$

which corresponds to

or

$\simeq 8$ observing days $/ \mathrm{yr}$

$\simeq 60 \mathrm{~h}$ of dark time $/ \mathrm{yr}$

\footnotetext{
${ }^{a}$ This table was extracted from "Potential NASA Scientific Missions: Reference Model Only-Not a NASA Plan-1973."
}

struments. We hope that every effort will be made to reserve $100-200 \mathrm{~kg}$ of weight, a few cubic feet of rack space, and very modest power allotments on every astronomy and physics sortie mission so that these and other small instruments may have as many flight opportunities as possible. Such a policy could have only a minor effect on the primary payload but a major effect on the science done.

If this mission model represents the maximum number of flights likely to be available for uv and optical astronomy, we believe that the payload distribution is reasonable.

\section{SUMMARY AND RECOMMENDATIONS}

\section{A. Utilizing the Shuttle for Optical and Ultraviolet Space Astronomy}

Very-high-resolution images in visible and ultraviolet wavelengths will be required to answer many of the most vital questions concerning the nature of our solar system, the evolution of stars and galaxies, and the structure of the universe. Spectroscopy, photometry, and polarimetry, particularly in the ultraviolet, will also be needed. Unfortunately, the earth's atmosphere severely limits the sharpness of astronomical images and is completely opaque to most ultraviolet wavelengths. Consequently, astronomical instruments in space are 
absolutely vital to the discipline. Among proposed space telescopes, the Large Space Telescope (LST) is outstanding in its ability to obtain observations of unprecedented quality and importance. It ranks as the single most important program in optical and ultraviolet space astronomy. Planned as a permanent 3-m astronomical observatory, the LST will utilize the capability of the Shuttle to provide servicing in orbit or return to earth.

Most optical and uv space-astronomy telescopes are technically more suited to the free-flying mode than to any other Shuttle mode. Generally, tight tolerances on the allowable guidance errors, thermal excursions, and contamination limits are more easily met in the freeflying mode. Most importantly, the free-flying mode offers far more observing time than does the sortie mode. However, several important smaller instruments are well suited to the sortie mode.

Besides serving as a booster with a large payload capacity, the Shuttle offers the extremely valuable advantage of manned assistance in deploying a free-flying spacecraft. In addition, the Shuttle provides the ability to periodically update and maintain the telescope in orbit. It also has the capability of returning an astronomical telescope to the earth for major refurbishment. However, we are concerncd that the latter option will result in free-flying spacecraft spending a large fraction of their time on the ground undergoing modifications and awaiting launch. We, therefore, believe that the former option should be used for instrument replacement and that free-flying satellites be returned to earth only when absolutely necessary.

We recommend that the Shuttle be designed to facilitate instrument replacement in orbit. In this connection, we also recommend that all free-flying astronomical telescopes be designed to make it as easy as possible for a space-suited astronaut to replace instruments and to make minor adjustments and repairs.

There are situations for which the sortie mode is highly advantageous. For example, imaging over large fields of view using photographic film (directly or via electronography) seems preferable in the sortie mode, where frequent manned access is possible. Although it would be possible to conduct a photographic survey with a freeflying telescope that is visited about once per year, the difficulties of protecting against film fogging due to charged particles, against systematically overexposing or underexposing, against misfocusing, and against film jamming are sufficiently large to warrant use of alternate approaches if possible. 
We believe the best approach to optimize the scientific returns is to lengthen the 7-day baseline sortie mission as much as possible. Since at the moment we cannot identify an overriding need for the Spacelab for astronomy experiments, we suggest that the weight penalty in expendables for longer missions be compensated by eliminating the Spacelab, utilizing only the Shuttle bay. In this approach, manned attendance would require wearing spacesuits. In the absence of a malfunction, we currently estimate that about one extravehicular activity per week should be sufficient. We recommend that the Shuttle be designed to facilitate the longest possible sortie missions.

Relatively simple telescopes requiring only the basic Shuttle guidance $(<0.1$ degree of arc) or moderately improved guidance $(\sim 1$ sec of arc) provided by a compact general-purpose stabilized platform provide another situation where the sortie mode can be advantageous. They could be controlled from ground stations, and data could be telemetered in the same way as for free-flyers. Contained in coarse gimbals of adequate angular range, they could be launched whenever additional space is available. Alternatively, there are several small telescopes with low guidance requirements that could be operated effectively through an airlock in the Shuttle cabin.

For the most part, we have not been able to identify any technical reasons that would preclude operation of astronomical telescopes in the sortie mode. However, there are some problems that we wish to emphasize. One of these problems is the increased complexity that will result if continuous command and telemetry are not available. We, therefore, recommend that a Tracking and Data Relay Satellite be available for astronomical space telescopes, particularly those utilizing the sortie mode.

Optical and uv payloads are sensitive to contamination by the Shuttle. Inadequate control could prevent execution of the projects investigated in this study. We, therefore, recommend that contamination be kept paramount among Shuttle design and operation considerations and that quantitatively acceptable contamination levels be established for the instruments proposed.

Many astronomical sensors would benefit from an equatorial orbit that avoids the South Atlantic Anomaly, thereby reducing the background of energetic particles. These charged particles will cause a noise background against which it is very difficult to shield or compensate. We recognize that the achievement of an equatorial orbit is difficult, unless an equatorial launch site can be developed. We rec- 
ommend that additional consideration be given to the problem of obtaining equatorial orbits.

One of the most exciting long-range prospects for astronomic research is the possibility of using the Shuttle to assemble very large telescopes and other instruments in orbit. We recommend that the Space Shuttle be designed to facilitate major assembly operations in space.

\section{B. Supporting Research and Technology}

The potential of the Shuttle for performing a large amount of space astronomy will not be borne out unless the proper telescopes and instruments are available when the Shuttle is ready to fly. At minimum, a vigorous supporting research and technology program is now required.

A promising imaging technique is electronography, for which fields of only a few centimeters diameter are currently available. For some applications, the required camera is one in which the image is read out electronically, thereby avoiding the use of film in orbit. We recommend that imaging cameras of large surface area $(20 \mathrm{~cm} \times$ $20 \mathrm{~cm}$ ) and high quantum efficiency be developed.

Many of the constraints on the Shuttle cannot be accurately specified until more detailed studies of astronomical payloads have been performed. This is particularly true of the thermal requirements. We, therefore, recommend that a thermal design study be made of a suitable sortie-mode telescope. 


\section{7 \\ Solar Physics}

\section{SOLAR-PHYSICS OBJECTIVES AND OVERALL PLAN}

The outstanding scientific problems in solar physics derive their significance as much from their intrinsic interest as plasma phenomena of extreme complexity as they do from their importance for the study and elucidation of a range of basic questions arising in our efforts to understand the physical universe.*

In summary, these problems center around (a) the origin of solar activity and the mechanisms underlying its various manifestations (especially flares), (b) the nature and origin of the mass and mechanical energy flux from the sun, and (c) physical problems of broad significance that can only be studied in the sun. We discuss these broad areas below, giving particular emphasis to the progress to be anticipated from solar observations during the Shuttle era.

\section{A. Solar Activity}

The study of the formation, heating, and long-term development and decay of active regions requires spatial correlation of observations made over a broad spectral range and over consecutive periods of a few days. For example, in order to study the interaction of rising magnetic fields with the plasma of the solar photospheric layers, long-term time-lapse observations with high spatial resolution in the visible portion of the spectrum are needed of velocity fields, smallscale magnetic fields, and features reflecting different temperature and density conditions. These observations must be correlated with

\footnotetext{
*In considering the scientific motivation, we have drawn heavily on the reports of the NASA Payload Planning Working Group (Blue Book) on Solar Physics and of the ESRO-PASOL Group and particularly on the discussions of those problems that they believe should consume a major fraction of the best efforts in solar physics through the first decade of the Shuttle era.
} 
the uv and x-ray observations of the same areas to yield parallel data on the higher levels in the sun's atmosphere-the chromosphere, transition region, and inner corona. Data show the spatial structure of active regions to be extremely complex and to change completely in the higher layers, where the magnetic field dominates; however, the limited spatial resolution currently available severely restricts our ability to interpret such data fully. The evolution of activity and the details of magnetic-field development will almost certainly depend on magnetic-field measurements made with high spatial resolution and extending over periods of a week or more.

Little is known about the impulsive nonthermal phase of flare development during which energy is released and charged particles accelerated to very high energies. We would like to know the location of the primary acceleration, the magnetic- and electric-field configurations, and the time sequence of the energy release processes. X-ray and radio-wave observations provide essential information on the energetic electron population of a flare, while the white-light, gamma-ray, and neutron emission give clues to the acceleration of protons. Direct measur $\bullet$ ment in space of the isotopic content of energetic flare particles promises to add still another insight into the acceleration, containment, and release of charged particles. Because theory suggests that the energy release and subsequent thermalization must take place in an extremely small-volume, high-temporal and -spatial resolution is essential.

Another area of current interest is the state of an active region prior to the occurrence of a flare. There are periods of rapid magnetic change in an active region during several hours or days prior to a large flare, during which time the $\mathrm{x}$-ray, xuv, and radio emission tend to increase in intensity. Accelerated particles of comparatively low energy are observed to escape from the buildup area into interplanetary space. This, with many other aspects of the buildup, is not understood, and further observations of particle densities, fluxes, temperatures, and magnetic fields-and the associated time variations-are needed.

\section{B. Energy and Mass Flow in the Solar Atmosphere}

The mechanisms that produce the large departures from radiative equilibrium that characterizes the chromosphere and corona are not understood. Compelling theoretical and observational evidence suggests that these levels are heated by mechanical disturbances such as acoustic, magnetoacoustic, and possibly gravity waves originating in 
the subphotospheric convection zone. The principal mechanism has not been identified in spite of the fact that recent years have produced a weaith of data on the temperature structure of the chromosphere-corona transition region as well as microscopic motions in the lower atmosphere.

Future work must provide a complete specification of the temperature, density, velocity structure, and magnetic field over the entire atmosphere from the photosphere out into the lower corona. Because these layers contain an intricate fine-scale horizontal structure, closely associated with the concentration of the magnetic field into small columns, high spatial resolution at all wavelengths is essential. Without such resolution the critical effects of the channeling of the mechanical energy flux by the magnetic field cannot be determined.

The flow of mass and energy in the solar atmosphere continues into interplanetary space in the form of the corona and solar wind. The magnetic field plays a crucial, if incompletely understood, role in modulating the flow of the material and imprinting an intricate density, temperature, and velocity structure on the plasma as it rushes out from the sun. Space probes have measured these at $1 \mathrm{AU}$; however, the connection between these observations and structures in the inner corona is just beginning to be established. Surprises, such as the recent realization that most of the solar wind originates in quite undistinguished regions of the corona, where the magnetic field is weak and open and the density is low, can be expected to be frequent and to lead to exciting revisions of our ideas on the structure of the outermost atmospheres of the sun and stars.

Understanding these processes requires a complete specification of the density, temperature, and magnetic field in the corona and solar wind, with good temporal and spatial resolution, so that a full threedimensional model can be established. Since the medium is continually evolving, synoptic observations are necessary to describe the influence of activity in the lower atmosphere on the upper levels. Moreover, high time-resolution measures are required to investigate the response of the corona-solar wind plasma to solar flares. A variety of tools will be required. Spaceborne coronagraphs have demonstrated their power on the Oso and ATM; however, these data must be supplemented by $x$-ray, euv, ground-based radioheliograph and coronagraph, spaceborne radiospectrographs, and in situ solar-wind measures if a complete picture is to be obtained. We would particularly stress the need for coordination of ground and space observations for incisive attacks on particular scientific objectives. 


\section{Physical Problems of Broader Significance}

Solar activity originates below the visible levels of the solar atmosphere; our knowledge of the structure and dynamics of the interior is, at best, provisional. Models provide a basis for understanding the most obvious properties of the sun-its mass, radius, and luminosityand show that the presence of a chromosphere and corona depends on the existence of a convection zone, some of whose characteristics are reflected in the photosphere. Similar models applied to other stars provide insight into their evolution, variability, and the processes of element synthesis.

In all these investigations, comparison with the sun furnishes a critical test; several tests lead to only a qualified confidence. For example, the currently accepted solar models predict a neutrino flux well in excess of the measured upper limit. Also, models incorporating convection in a rotating sun are not yet sufficiently advanced to explain the observed differential rotation of the photosphere and the characteristics of the solar magnetic cycle. With these more obvious features of the sun unexplained, it is small wonder that more subtle questions such as the nature of supergranulation cells, solar oblateness, and the role of the solar wind in the angular momentum history of the sun remain subjects of speculation. Likewise, broader questions regarding the presence of similar phenomena on other stars remain uncertain. The constancy of the solar "constant"-a fundamental parameter in all studies involving terrestrial climate-appears to be an article of faith.

It is clear that little progress can be made until our ideas concerning the role of turbulent convection in determining the structure of the sun and its interaction with solar rotation are clarified. Here, a fundamental advance in the theory of turbulent convection beyond the currently used mixing length models is essential. The application of modern computational tools to these problems will be essential but may be misleading without this fundamental knowledge.

A directly related problem is the operation of the solar dynamo and the production of the solar magnetic cycle. If the investigations mentioned earlier are successful, there should be no lack of fundamental knowledge that would impede progress in the study of the solar cycle. Advancing our knowledge of the stability of the sun, and the consequent implications on the solar constant, and the neutrino deficit must proceed in concert with these studies. Although progress can be made using the current models, the stability of the sun is most 
certainly dependent on the coupling between the energy generating core, the radiative envelope, and the convection zone. Since the characteristics of these zones are not fully known, the presence of a solar variability independent of the magnetic cycle remains uncertain.

\section{Relation of Solar Physics to Other Disciplines}

The outstanding problems discussed above have an importance far beyond solar physics. Thus, once the processes of mechanical energy production, transport, and dissipation are understood, observations of stellar chromospheres and coronae could be used for further studies of stellar structure and evolution, since the extent of the subphotospheric convection surely varies with spectral type and class. Since it seems clear that small-scale photospheric features are associated with production of the mechanical energy that heat the chromosphere and corona, such motions and fine structures should exist also in the atmospheres of stars exhibiting chromospheric features; the interpretation of the spectra of such stars must rest heavily on the solution of the mass and energy-flux problem of the solar atmosphere.

Continuing studies of the solar wind will find application in understanding stellar winds and mass-loss mechanisms. The process whereby the solar wind removes angular momentum from the sun, thus slowing down solar rotation, is basic to an understanding of the origin and evolution of the solar system and of other stars and planetary systems. This mass loss is important in determining the composition of the interstellar medium and interplanetary plasma.

Solar flares exhibit a broad range of high-energy processes, including the generation of hard cosmic rays and associated radiation, extending over the spectrum from gamma rays to radio wavelengths. The sun provides an opportunity for detailed study of the interaction of high-energy particles and magnetic fields, since both of these characteristics can be measured directly. Such studies have clear and direct relevance to the study of other energetic objects in the universe. Similarly, the study of solar-active regions and the long-term interaction of the solar plasma and magnetic fields should increase our understanding of the coupling between solar convection, differential rotation, and the loss of angular momentum, as well as cycles of stellar activity.

We can look to a continuing stimulation of many other areas in astrophysics coming from attempts to understand the complex questions posed by solar physics. As a single example. important 
studies of atomic processes in low-density plasmas have followed efforts to account for the physical state of the solar atmosphere.

Finally, as man's technical achievements mount, the importance of a detailed understanding of solar-terrestrial effects will grow. The influences of solar activity on the upper terrestrial atmosphere are well documented, if insufficiently understood. The solar wind stands out as the principal modulator of the magnetosphere. Significant progress has been made in our ability to predict the occurrence of major flares, and a capability for accurate prediction would have economic benefits and may determine the extent to which man can work in space above the atmosphere. Finally, a possible link between solar activity and large-scale terrestrial weather patterns suggests potential significance of solar space studies to all mankind.

\section{PROFILE FOR A BALANCED PROGRAM IN SOLAR ASTRONOMY}

With the above objectives as guidelines, we have developed a set of goals that we believe would provide a well-balanced program in solar astronomy through the 1980's. These are outlined briefly below; more detailed descriptions are set out in Section III.

\section{A. Spaceflight Aspects}

A solar maximum satellite for the 1978-1979 period would allow, in conjunction with ground-based studies, an incisive approach to the study of solar activity in its various manifestations. Furthermore, the basic spacecraft, through Shuttle recovery, relaunch, and revisit, would provide a free-flying payload for long-duration solar experiments in the $1980^{\prime} \mathrm{s}$.

Basic instrumentation for a Shuttle Sortie Solar Observatory (SSO) falls into two categories. First we envisage a set of major telescopes optimized for different wavelength regions and feeding interchangeable specialized instruments (spectrographs, direct cameras, magnetometers). Second would be a versatile, fine-pointed platform for mounting special-purpose instruments that may be incompatible with the larger feed telescopes or not require their power-examples are coronagraphs and polarimeters. The larger system, at least, should be started soon to provide the opportunity of studying problems of solar activity with more powerful instruments (even if narrower in scope) than those on the free-flying satellite. 
While the smaller fine-pointed platform should be developed on a single pallet as a module for the sortie solar observatory, we also see an attractive possibiiity in its use to carry payioads on a standiby basis-an opportunity whereby an available payload could be carried on an otherwise unfilled sortie mission. This concept needs study to determine its feasibility.

Because the ultimate observational needs of solar astronomy may eventually require a free-flying Large Solar Observatory, we recommend that the National Academy of Sciences convene a panel of scientists to investigate all aspects of the need and specifications for, and use of, such a facility.

\section{B. Other Necessary Components of a Balanced Program}

\section{OTHER SATELLITE OBSERVATIONS}

A coordinated approach to a variety of solar-physics problems requires that numerous observations be made at the same time. Many of these must be made from spacecraft flying outside the magnetosphere. Particularly relevant are very-low-frequency radio measurements, in situ observations of solar-wind plasma and magnetic field, and high-energy particle measurements. Specific attention should be given to the scheduling of launches of such payloads to optimize the scientific returns coordinated with the solar Shuttle missions.

\section{DATA ANALYSIS AND THEORETICAL STUDIES}

Adequate and sustained support for the analysis of experimental data, as for parallel theoretical studies, is imperative if the data are to be used for increasing our understanding of the sun. This support must be provided for at the earliest planning stages.

\section{GROUND-BASED OBSERVATORIES}

In the Shuttle era, solar astronomy will make increasingly heavy demands on the ground-based observatory capabilities at optical and radio wavelengths. The multiparameter observational detail required in order to develop an understanding of solar phenomena necessarily results in the integration of data from a broad variety of sources. Furthermore, as the understanding of basic solar processes unfolds, it is necessary to maintain the ground-based as well as the space-based solar capabilities at the forefront of technological sophistication. 


\section{ROCKETS AND BALLOONS}

The return from the use of rockets and balloons for solar studies has far more than justified the cost. With the augmented payload capability and excellent pointing controls now available, these experiment platforms continue to provide an important part of a balanced solar-astronomy effort. As solar astronomy enters the Shuttle era, it is important that the rocket and balloon programs be continued, both for original solar studies and for the development of Shuttle-compatible instrumentation. Only after we are well into the operational Shuttle era will experience be available to permit a reassessment of the role of the rocket and balloon capability for solar studies.

\section{SUPPORTING RESEARCH AND TECHNOLOGY}

In the past, the SR\&T program in NASA has been pivotal in developing and maintaining the solar-astronomy program and has underlain the excellent progress in understanding the sun and its influences. Sadly, the decrease in this type of funding in recent years has not only had an impact on established research efforts but has curtailed the investigation and development of new ideas that represent investment in the future. We most urgently recommend that SR\&T support be maintained and augmented as a balanced part of the total NASA program.

\section{I. M I S S I O N O D E L}

Table 17 is the mission model that we recommend to achieve the goals outlined. It is designed to meet the anticipated needs of U.S., European, and other scientific groups. It envisages a launch of the Solar Maximum Mission (SMM) in 1977/78, a schedule of sortie missions starting in 1980 with a buildup to four missions a year from 1983, and, starting in 1980, an annual schedule of new flights, revisits, and refurbishments of the free-flyer spacecraft originally designed for the SMM. A certain fraction of these would carry new payloads; some would simply replace consumables on the spacecraft. The initiation schedule (SMM in 1978, first sortie at the end of 1979) is set by the coming solar maximum and is more fully documented elsewhere.

Over a 10-year period, the total number of dedicated missions will be 34 including the following: Solar Telescope Cluster (STC), 17 flights; Large Fine-Pointed Platform (LFPP), 17 flights; High-Energy 
TABLE 17 Mission Model

\begin{tabular}{|c|c|c|c|c|c|c|c|c|c|}
\hline \multirow[b]{2}{*}{ Item } & \multicolumn{9}{|c|}{ Years } \\
\hline & 77 & 80 & 81 & 82 & 83 & 84 & 85 & 86 & 87 \\
\hline $\begin{array}{l}\text { Missions on which } \\
\text { SSO is prime payload }\end{array}$ & & 2 & 2 & 2 & 4 & 4 & 4 & 4 & 4 \\
\hline $\begin{array}{l}\text { Missions flying the } \\
\text { SFP only which are } \\
\text { not included above } b\end{array}$ & & 1 & 1 & 1 & 3 & 3 & 3 & 3 & 3 \\
\hline $\begin{array}{l}\text { Sortie flights of } \\
\text { opportunity for the } \\
\text { SEPPC }\end{array}$ & & 1 & 3 & 2 & 2 & 2 & 2 & 2 & 2 \\
\hline Solar Maximum Mission ${ }^{d}$ & 1 & & & & & & & & \\
\hline (Large Solar Observatory) & & & & & & & 1 & & \\
\hline
\end{tabular}

${ }^{a}$ These are dedicated missions for solar physics only. They might carry into space one of the four following packages:

$\begin{array}{lr}\text { Payload } & \text { Average Annual Rate } \\ \text { STC + SFPP } & 2 \\ \text { LFPP + SFPP + FF } & 1 \\ \text { LFPP + SFPP + HESP } & 1\end{array}$

${ }^{b}$ These are missions for which solar-physics payloads carried by the SFPP will fly with payloads belonging to other disciplines.

${ }^{c}$ These numbers assume that the number of rocket payloads launched per year in the Shuttle area will be maintained at the present level of activity in the United States, Europe, and Japan.

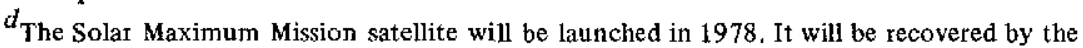
Shuttle in 1980, refurbished, equipped with updated instruments, and launched by one of the dedicated missions once every year.

Solar Package (HESP), 7 flights; Free-Flyer Satellite (FF), 10 flights (or revisits); Small Fine-Pointed Platform (SFPP), 34 flights. The total number of missions flying the SFPP only over 10 years is 24 ; the total number of flights of opportunity for the SFPP for the same petiod is 21 .

The sounding-rocket program goal of 25 flights per year would continue through 1982 at least; its continuation beyond that must be a subject for study over the coming few years as the Shuttle sortie capability becomes more defined.

Also envisaged is a Large Solar Observatory program with annual revisits, although the need for closer definition of this program is reflected in our parenthetical entry of this item in Table 17.

\section{A. The Pre-Shuttle Solar Maximum Mission}

Because of the timing requirement imposed by the 11-year solar 
cycle, we regard a free-flying satellite, with a carefully coordinated complement of instruments for the study of the next solar maximum, as the highest immediate priority item for solar physics.

Solar activity may be expected to return in 1977 and reach a peak approximately in 1979, with the likelihood of observing major flares in a seven-day mission decreasing rapidly after 1981 . We believe that an immediate start on this project is required in order to use this opportunity, which will not be repeated until 1990.

The study of solar activity, especially flares, requires a wide range of instruments to cover the electromagnetic spectrum from visible wavelengths to several $\mathrm{MeV}$, where solar nuclear gamma-ray lines have been observed. In particular, the study of the effects of nonthermal particles at high $\mathrm{x}$-ray and gamma-ray energies requires specialized instrumentation that was not available during the last maximum in 1968 but that is now within the state of the art. Further, the high resolution that will become available simultaneously in spatial and spectral properties of the thermal flare plasma with the generation of x-ray and euv spectroheliographs, which we believe can be developed in ample time for the Solar Maximum Mission (SMM), will allow studies that can be achieved in no other way.

\section{DESIGN OF THE SPACECRAFT}

The SMM presents an opportunity to develop a standard solar free-flying observatory for the Shuttle era. The SMM satellite concept developed by the Goddard Space Flight Center seems to provide an excellent basic capability that can support the pre-Shuttle SMM and that has the growth capability to accommodate instruments of the class of the Shuttle Sortie Observatory in a free-flying mode. The SMM concept envisages a Delta-launched satellite with the capability of fine pointing of some $500 \mathrm{~kg}$ of instruments at the sun. The SMM concept will, by 1977 , provide a pointed payload four times greater than OSO-I, with over twice the power, more than 10 times the viewing area for pointed-instruments, enhanced pointing accuracy, and comparable telemetry and command capability. Such capabilities, combined in a single spacecraft, will make it possible to achieve the scientific objects with a low-cost approach. We strongly endorse the SMM concept, not only for the pre-Shuttle SMM, which is of paramount importance, but as the basis for a flexible future series of the Solar Free-Flying Observatories. 
Alternative concepts for the spacecraft are not necessarily ruled out; however, it would be essential that the following requirements be met to provide a viable system:

1. The spacecraft should be available for use at the next solar maximum in 1977-1979.

2. The pointing stability should be better than $1 \mathrm{sec}$ of arc over a period of $5 \mathrm{~min}$.

3. The spacecraft should be designed as a revisitable and reusable free-flyer throughout the Shuttle era.

\section{SELECTION OF PAYLOAD FOR SMM}

While we recognize that there may be strong constraints on funding experiments for this mission, it is obvious that the best science, which must be the principal objective of the mission, will not be accomplished by simply reflying experiments that have already successfully returned data, simply in the name of economy. The design of the spacecraft and the ample size and weight provision should allow new experimental approaches. We, therefore, urge that NASA ensure that experiment selection follow the proven method of open competition and impartial review.

\section{B. Use of the Space Shuttle for Solar Research}

The following sections summarize our recommendations for use of the Shuttle as a base for solar experiments and as a transportation system for free-flying satellites.

\section{SORTIE MODE}

We have identified four basic solar-physics sortie payloads, which can provide the flexibility to accommodate the broad range of instrumentation required to implement the observational program outlined. Two payloads have been identified as basic multiuse facilities: the Solar Telescope Cluster, which provides a basic set of optical feeds for a variety of imaging, spectroscopic, and polarization studies between $8 \AA$ and $10000 \AA$; and a High-Energy Solar-Physics Package, which can carry out similar studies between $1 \mathrm{keV}$ and 100 $\mathrm{MeV}$ and with the high time resolution necessary to study nonthermal events. We have also defined two different size fine-pointed platforms that can accommodate a variety of specialized instruments. These four basic experiment packages are described in this section, and representative instrumentation is presented in Appendix A. 
TABLE 18 Characteristics of the Solar Telescope Cluster

\begin{tabular}{|c|c|c|c|c|c|}
\hline $\begin{array}{l}\text { Wavelength } \\
\text { Range }\end{array}$ & $\begin{array}{l}\text { Spatial } \\
\text { Resolution } \\
\text { (sec of arc) }\end{array}$ & Type & Aperture & Length ${ }^{a}$ & $\begin{array}{l}\text { Collect- } \\
\text { ing Area }\end{array}$ \\
\hline $1200 \AA$ & $\begin{array}{l}0.1 \text { at } \\
5000 \AA\end{array}$ & Gregorian & $\begin{array}{l}100-\mathrm{cm} \\
\text { / } / 5 \text { primary } \\
\text { f/35 overall }\end{array}$ & $6 \mathrm{~m}$ & $7500 \mathrm{~cm}^{2}$ \\
\hline $300-1600 \AA$ & 0.5 & $\begin{array}{l}\text { Normal-incidence } \\
\text { mirror }\end{array}$ & $\begin{array}{r}40 \mathrm{~cm} \\
/ / 10\end{array}$ & $5 \mathrm{~m}$ & $1250 \mathrm{~cm}^{2}$ \\
\hline $140-600 \AA$ & 0.5 on axis & Wolter type I] & $80 \mathrm{~cm}$ & $5 \mathrm{~m}$ & $1500 \mathrm{~cm}^{2}$ \\
\hline $8-300 \AA$ & 1 on axis & Wolter type I & $80 \mathrm{~cm}$ & $5 \mathrm{~m}$ & $\begin{array}{l}450 \mathrm{~cm}^{2} \\
(\lambda>20 \AA)\end{array}$ \\
\hline $1-40 \mathrm{keV}$ & 4 & Oda collimator & $50 \mathrm{~cm}$ & $6 \mathrm{~m}$ & $1000 \mathrm{~cm}^{2}$ \\
\hline
\end{tabular}

(a) SOLAR TELESCOPE CLUSTER Since radiation emitted over the entire wavelength range from below $1 \AA$ into the millimetric range arises in different height and temperature regimes in the solar atmosphere, a battery of telescopes is required to carry out the needed research. Our recommendation for such a battery is summarized in Table 18, while a brief description of each component appears below. (Alignment of the entire battery on a given solar feature to within $1 \mathrm{sec}$ of arc, as well as independent pointing of individual telescopes to any part of the solar disk, is required.) These specifications are presented as our desired goals; we well recognize that funding or technical constraints may delay the deployment of some elements of the ultimate cluster.

(i) OPTICAL TELESCOPE A 1-m-diameter, $f / 35$, diffractionlimited telescope yielding angular resolution of about $0.1 \mathrm{sec}$ of arc at $5000 \AA$ is desired. A design goal should be to extend the technology of surface finishing so that the system can operate with similar angular resolution down to Lyman- $\alpha$. Such a system has the advantage that it builds upon the technology of intermediate-size systems of $65-\mathrm{cm}$ aperture, which are planned for flight in the next several years in stratospheric balloons, while representing a reasonable advance of performance. As a general-use system, such a heliograph should be equipped with a variety of final image magnifications as well as auxiliary devices such as spectrographs, filters, polarimeters, cameras, and magnetographs for investigations in the wavelength range from $1200 \AA$ to $1 \mathrm{~mm}$. Such devices should be designed for modular installation of various combinations for 
differing scientific objectives. The use of such a telescope for nonsolar observations should be considered in its design.

(ii) EUV TELESCOPE This instrument should be designed for maximum collecting area and greatest possible efficiency (i.e., minimum number of reflections), consistent with use of highefficiency stigmatic spectrographs as subsidiary instrumentation. It should cover the range 300 to $1500 \AA$ with normal-incidence optics, designed to produce image quality better than $0.5 \mathrm{sec}$ of arc within $1 \mathrm{~min}$ of arc of the optic axis. Such a system could then feed, for example, a stigmatic spectrograph of $\sim 1-\mathrm{m}$ focal length, which also, by rocking the objective by $\pm 15 \mathrm{sec}$, would produce high-resolution spectroheljograms in a variety of lines. Other possible instruments that could be placed at the focal plane include (1) narrow-band filters (e.g., for $L y-\alpha$ ); (2) special-purpose spectrometers for measuring velocities, particular line ratios, or line profiles; and (3) polarimeters.

Although the efficiency of normal-incidence optics drops seriously in the far uv, it is important that every attempt be made to extend the spectral ranges to include the strong He II $304 \AA$ line. Consideration should also be given, however, to extending the long-wavelength limit of the grazing-incidence Wolter type II telescope described below to overlap the $300-1600 \AA$ range.

(iii) X-RAY TELESCOPES Adequate coverage of the shorter wavelengths will require three individual telescopes. Two grazingincidence imaging telescopes will operate longward of about $8 \AA$; the shortest wavelengths are probably best covered by a nonimaging mechanical Oda collimator, although this possibility needs further study.

The characteristics of the individual Wolter-type reflectors would be tailored to provide the maximum available effective aperture for each range. The short-wavelength limit of this system is strictly set by the brightness of the source; for solar flare studies, this telescope should be usable down to approximately $2 \AA$.

For imaging studies, the entire collecting area is available, and a spatial resolution of $1 \mathrm{sec}$ of arc (or better) should be attainable over a 1-2 min of arc field. For spectroscopic studies, the different optics allow separate spectrometers to work in the wavelength ranges from $8 \AA$ to $\sim 50 \AA$ and from $40 \AA$ to $\sim 300 \AA$. Limitations of collecting area may require spectroscopic or polarization observations to be carried out at lower resolution. 
The Oda collimator covers wavelengths too short for effective imaging, even at grazing incidence. It will require devices to raster or scan its field of view over the regions of interest; it normally will be used to feed spectrometers or polarimeters.

(b) COARSE-POINTED HIGH-ENERGY MEASUREMENTS Comprehensive measurements of the characteristics of X-ray, gamma-ray, and neutron emission from the flaring and nonflaring sun would give insight into the triggering mechanism and total energy content of a flare (in conjunction with other measurements) and into the acceleration, containment, and release of charged particles. The recent oso-7 discovery of flare-excited nuclear gamma-ray lines is indicative of the expected new results from future high-energy studies. Use of the sortie mode for these high-energy experiments permits observations to be made simultaneously with longerwavelength experiments and accommodation of high weight and data rates. These ends could also be accomplished by an appropriate scheduling of free-flyers. The cost-effectiveness of both modes should be investigated.

The intensity distribution and its variation with time and position should be measured for photons in the spectral range of 0.001 to above $10 \mathrm{MeV}$. The flux, spectrum, and time history of neutrons should be measured; a representative set of instruments is specified in Appendix A.

The measurements taken during flares will be of great significance when compared with simuitaneous radio spectral and spatial measurements and with solar-particle measurements obtained by other spacecraft.

(c) GENERAL-PURPOSE, FINE-POINTED PLATFORMS Several scientific disciplines will require oriented platforms for the Shuttle sortie mode. To carry the full range of possible solar experiments, we recommend that two pointed platforms be developed; the stability requirements for both platforms are $1 \mathrm{sec}$ of arc, but they differ in size. The smaller fine-pointed platform should accommodate instruments up to $2 \mathrm{~m}$ long, and might, for example, be based on a half-pallet section. This unit could be flown on sortie launches with only a limited amount of unused space or load capacity. It represents an important component of the proposed facilities; it will be ideal for carrying the type of experiment now flown on rockets. It will accommodate larger and heavier payloads than do present rockets and will permit the evolution of current rocketborne experiments. Its 
early deployment would allow smaller scientific groups to participate in early sortie flights.

The larger fime-pointed plation shoula accommodate instruments up to $2 \mathrm{~m}$ in diameter by $4 \mathrm{~m}$ long and weighing up to $3000 \mathrm{~kg}$. As part of the Sortie Solar Observatory it would, for example, carry large special-purpose instruments not included in the Solar Telescope Cluster or a problem-oriented package of several experiments of a size intermediate between current rocket or OSOtype experiments.

The design of these platforms must be such as to allow payload development with minimal interaction with the Shuttle itself. A clean interface for power, thermal-control, data-transfer, and experiment control functions is important.

(d) DEPLOYABLE RECOVERABLE FREE-FLYER A semiautomated free-flying pointing platform based on an evolution of the OSO series or the proposed SMM satellite is needed for some observational programs with duration well in excess of that of a single sortie flight. Additionally, some experiments require a higher freedom from contamination than is available on the Shuttle.

A deployment recovery mode is likely to be the most efficient way of serving this class of platform since (i) there is no need to build a new spacecraft for every mission; (ii) the instrumentation can be returned, updated, recalibrated (with a high degree of confidence never reached up to now), and flown again; and (iii) spacecraft consumables and components can be replenished, repaired, or replaced within a short lapse of time.

The SMM satellite should be designed with these needs closely in mind.

\section{(e) OTHER ASPECTS}

(i) SOLAR FLIGHTS OF OPPORTUNITY The payload carrying capability of the Space Shuttle may be used to permit observations from space in a piggyback mode at modest cost and with great flexibility. In this mode, experiments of an exploratory or developmental nature may be carried out on a space-available basis.

For solar studies, this mode would make use of the generalpurpose fine-pointed instrument platform described above. It is desirable that this platform be built as a modular independent facility to permit mounting into the Space Shuttle with minimum interference to the prime Shuttle mission. For effective and low-cost 
utilization of this mode, it is essential that this facility have clean and standardized interfaces with the Shuttle orbiter.

We strongly recommend that the experiment accommodation management of this facility be as direct and informal as possible in order to promote maximum utilization at minimum cost and lead time.

(ii) CALIBRATION Accurate instrument calibration is especially critical for solar observations whose analysis demands high photometric accuracy. For example, a powerful method of determining density or temperature of the solar plasma makes use of the accurate measurement of ratios of spectral line intensities - often at widely separated wavelengths-and for this the absolute values of these intensities are essential.

The Shuttle sortie mode is well suited for achieving accurate calibration since, in principle, instrument calibration can be monitored during operation and a thorough recalibration made immediately after flight.

(iii) THE ROLE OF MAN IN SOLAR SORTIE OBSERVATIONS The recent successful operation of manned space solar-astronomy experiments of ATM during the Skylab SL/2 mission has given needed perspective on the role of man in future sortie solar observations. The man-instrument interaction on ATM takes three forms: as an observer, as an operator, and as a technician.

As observers the $\mathrm{SL} / 2$ crew have shown themselves capable of educated and thoughtful choice of pointing coordinates within the solar features chosen for study and have made important real-time decisions such as when and how to observe transient phenomena such as flares. It is fair to say that the presence of educated observers at the telescopes has greatly enhanced the resulting data.

As operators the crew have skillfully initiated complicated observing sequences, many of which occurred out of reach of ground stations and therefore could not have been initiated from the ground. It is fair to note, however, that if the Skylab had been in continuous telemetry contact, these operations could have been. accomplished from the ground.

As a technician man has been essential in Skylab; the crew erected a thermal shield, deployed a faulty solar power panel, overhauled an inoperative stellar uv experiment, repaired faulty voltage regulators, cleared the optics of the ATM coronagraph, repaired faulty experiment doors, replaced two jammed film cameras, and returned exposed film to earth. It seems probable that the usefulness of man as a 
technician will continue to be paramount in the Shuttle sortie mode. There seem to be very strong reasons, however, for carrying out observationai and operational activities from the ground. These include the following:

1. Ground support of scientific operations can continue $24 \mathrm{~h}$ per day by rotation of ground personnel, thus substantially increasing the total observing time.

2. Consultations among a number of solar scientists on the ground before and during the observational sequences will improve the quality of the observations.

Reasonably high data rates would be required to operate the experiments from the ground. However, this capability would also permit returning all or a sampling of the data to earth in real-time or near real-time. This leads to a third advantage of ground-based operation.

3. Quick-look evaluation of the data within hours or at most a day of the observation will permit updating and improving observations planned for later in the same mission. Experience on OSO's and ATM have proven the worth of quick-look data evaluation for mission planning.

Therefore, we believe that it is important to provide the capability for ground-based evaluation, through use of a Tracking and Data Relay Satellite or other continuous high-data-rate system. One crew member should be thoroughly competent to make technical adjustments to the solar instrumentation; if he is also a competent observer, he might carry out observations directly as time permits, in close collaboration with ground-based colleagues.

\section{REQUIREMENTS IMPOSED ON SHUTTLE AND SPACELAB BY THE SOLAR PROGRAM}

\section{A. Contamination of the Optical Environment}

The Panel is concerned that the Shuttle may contaminate the local environment and the optical surfaces of many of the experiments because of the extensive use of volatile materials and the uncontrolled dumping of wastes. 
The Panel recommends that NASA establish a Shuttle Contamination Control Board to examine all materials, engineering approaches, and inflight procedures that may have implications for the contamination problem. Such a group could recommend modifications to assure that tolerable limits of contaminating gases and particulates are maintained. A similar Board operated for Skylab, and a considerable body of observational data on this problem will be available from Skylab.

\section{B. Scheduling of Solar Missions}

Because solar studies typically make use of many coordinated observations, it will be advisable to schedule solar sortie missions to coincide with supporting ground-based observations. This will in general be during May-September, as most major solar facilities are in the northern hemisphere and are located at sites where the skies are clearer during the summer than the winter months.

\section{Orbital Considerations}

Solar sortie missions will in general make use of orbits requiring minimum fuel consumption in order to maximize available payload weight.

Solar free-flyers should be put into orbits that will maximize recovery and revisit opportunities. Sun-synchronus missions for studies requiring continuous coverage are also possible.

\section{Tracking and Data-Relay Satellite System (TDRS)}

We believe that a data-relay system permitting nearly continuous contact with the Shuttle sortie is essential for maximum scientific productivity. Further, we believe that the Orbiter/TDRS wideband data link, which in the present mission model is regarded as optional, is indispensable to the effective use of the sortie mode and should be a part of the Shuttle program from the beginning.

\section{E. Payload Capacity}

It appears that the solar sortie will be limited by return payload weight for most missions, which will limit the ability to conduct coordinated experiments. We strongly urge that the weight landing 
capacity of the Shuttle be increased to as near the original goal as possible.

\section{F. Mission Duration}

Some solar missions will benefit greatly from longer missions, up to the full 30-day capability. The Shuttle should be designed to minimize the payload impact of such longer missions.

\section{G. Use of the Payload Specialist Station}

Flights of the Solar Telescope Cluster and other major solar payloads will utilize, on occasion, the payload specialist in an interactive role in the experiment. However, if the required console displays and controls are housed in a Spacelab pressurized module (as presently defined), it appears that the weight of that unit will seriously limit the size of the scientific payload and may, indeed, prevent flying the full Solar Telescope Cluster. Assuming that it is impractical to increase the permissible landing weight of the Shuttle, then the best solution seems to be to design the payload specialist console to allow adequate servicing of the scientific payload.

\section{H. Data and Control Interfacing}

It is recognized that the time available for payload integration with the Shuttle may be extremely limited. We suggest that these requirements may be met if the pallet itself includes a general-purpose computer of substantial capacity (e.g., 128 kbits of direct-access memory plus mass storage capability of at least $10^{10}$ bits) that is used for experiment control and data management and the payload specialist console serves primarily as a terminal for this computer. The console should also include video and CRT displays, the latter for display of information from the computer. Although most control functions would be derived from the pallet computer, it is advisable to have several analog servo-control circuits included in the console for instrument manipulation and limited analog readouts for critical experiment monitors.

With such a design, all experiment functions and computer software could be integrated and checked out using a Payload Specialist Console Simulator prior to mounting the pallet in the Shuttle. Also, this approach will minimize mission peculiar modifications of the Payload Specialist Station, requiring only that the terminalcomputer interface be standardized. 


\section{GENERAL CONSIDERATIONS}

\section{A. The Impact of Quality Assurance on Costs}

The Space Shuttle could substantially reduce the cost of transporting payloads to orbit, as well as increasing the number of flight opportunities. To take advantage of these opportunities, the cost per pound of payload must be substantially reduced. Part of this saving may be achieved by substantially streamlining the documentation and verification requirements of present-day quality assurance procedures.

We recommend that a panel of experienced Principal Investigators and satellite and experiment program managers from the various NASA Centers and from NASA Headquarters be, established to examine the problem of quality assurance in the Shuttle era and to make specific recommendations on procedures for sortie instruments and for instrumentation on free-flyers. We believe that the basic quality assurance approach recommended in the Shuttle sortie model presented by NASA is an excellent one and recommend that Principal Investigators work closely with NASA to implement this approach. We also recommend asking the proposed panel on quality assurance to consider the Solar Maximum Mission proposed for 1978, since this mission will be a prototype of the free-flyer of the Shuttle era.

\section{B. Convening of a Shuttle Experimentation Planning Committee}

Because the Shuttle and sortie laboratory are still in the planning stage, it is important to establish a continuing channel for exchange of information-e.g., payload accommodations, contamination control, and pointing requirements-between the scientific community and the Shuttle and sortie laboratory planners. Accordingly, we recommend that a committee of representative experimenters be set up for this purpose; this committee could be drawn, for example, from the existing U.S. and European working groups. We also recommend that these working groups be continued.

\section{Selection and Responsibilities of Scientists}

We consider that the successful construction and operation of individual instruments of any size is best accomplished under the supervision of a single responsible scientist. The process of selecting experiments must avoid conflict of interest, be open at all program 
phases, and must reflect the requirement that observing time and data are to be made available to guest investigators. A promising start in defining management responsibilities in this area is set out in detail in the report of the NASA Payload Planning Working Group.

\section{The Crucial Role of SR\&T Support}

Supporting research and technology provides, at modest cost, the basis from which flight programs grow. The Shuttle promises to provide a splendid opportunity for deployment of new and exciting instruments. To produce these in time for solar maximum, a start must be made now on instrument development. Because of the large payloads carried by the Shuttle, a substantial effort is needed, requiring a corresponding increase in SR\&T funding or special allocation of funds for Shuttle instrument development.

\section{RE COMMEN DATIONS}

1. The occurrence of solar activity presents a unique opportunity to investigate a broad variety of energetic astrophysical processes and, in particular, to study the role played by magnetic fields in such phenomena. For this reason, it is crucial to exploit the forthcoming maximum in solar activity (anticipated for early 1979); an equivalent opportunity will not be repeated until at least 1990.

We, therefore, recommend that the highest priority be given to the implementation of a Solar Maximum Mission ( SMM) satellite to be launched in late 1977 to observe the upsurge of solar activity and designed to permit uprating as a free-flyer in the Shuttle era.

2. The early data from the ATM have clearly demonstrated that instrumentation covering a wide range of the electromagnetic spectrum is essential for a broad attack on the fundamental problems of solar physics. This concept can be used to great advantage on the Shuttle because of its high-pay load and data-return capabilities. Such a wide variety of problems can be approached in this way that a series of missions is required, each having different specialized detectors at the focal planes of a cluster of generalized light collectors.

We, therefore, recommend that a flight program be initiated with the aim of development of a Shuttle Sortie Observatory consisting of (a) a solar telescope cluster of large collectors covering a wide range of the electromagnetic spectrum and designed to feed different focalplane instruments on different flights; (b) a small, fine-pointed platform for experiments of the rocket class; (c) a coarse-pointed package for high-energy solar measurements. 
3. Certain important needs of solar physics are not met by the Shuttle sortie. Among these are (a) longnterm, synoptic observations of such long-lived phenomena as active regions and coronal structures where moderate data rates suffice; (b) rare events, such as major flares, which can be studied only by long-duration observations; (c) contamination-free observations; and (d) observations for correlative purposes with observations from other spacecraft or from the ground. All these needs can be met by a free-flying spacecraft. The concept of periodic recovery, refurbishment, and instrument interchange on the SMM spacecraft offers an attractive, flexible, and inexpensive solution to this need.

We, therefore, recommend the creation of a solar free-flyer program based on Shuttle recovery and upgrading of the SMM spacecraft.

4. Certain important solar instruments such as coronagraphs and some special xuv devices require a large fine-pointed platform but are not adaptable to the general-purpose Solar Telescope Cluster.

We, therefore, recommend the development of a large finepointed platform to accommodate these larger instruments.

5. Considerable cost savings may be realized by developing instruments usable by different disciplines and programming observations so that some of the powerful hardware developed in one area of astronomy can be used for observations in others.

We, therefore, recommend that close attention be given at all planning stages to the possibility of development of modular instrument packages or interdisciplinary use.

6. The ultimate observational goals of solar studies make the eventual deployment of large instruments on a free-flying platform a most attractive possibility, particularly in view of recent spectacular Skylab, Oso, and ground observations. With the availability of the Shuttle to carry such large loads, the time is ripe to begin planning for such a program.

We, therefore, recommend that a panel be convened under the auspices of the National Academy of Sciences to study all aspects of a Large Solar Observatory (LSO).

7. Because of the novelty and complexity of the Shuttle operation, we recommend the establishment of a representative Shuttle experimentation planning board drawn from the disciplines to work closely with the Shuttle and sortie laboratory planners in defining experiment accommodations to be required.

8. Because of the severe weight penalty presently imposed by the use of the sortie laboratory module, we recommend that, as a priority matter, adequate payload specialist console space be provided 
along with sufficient and data storage in the orbiter/sortie pallet mode.

9. Because of the planned operational mode, detailed specifically in the above text, we recommend that the fundamental importance to solar-physics missions of a wideband Shuttle/TDRS relay satellite capability be kept closely in mind in all planning stages.

10. The extent to which the potential of the Shuttle is realized in advancing space science depends intimately on the degree of continued input of the scientific community, especially during the planning stages. The discipline working groups constituted by NASA have set a sound direction for such communication. We, therefore, recommend that a continued and close interaction between scientists and planners be recognized as an essential component in Shuttle development and that appropriate mechanisms (e.g., discipline working groups) be established to ensure this interaction.

A P P E N D I X. A

1. Representative Focal Plane Instrumentation for Use with the Solar Telescope Cluster ${ }^{a}$

\begin{tabular}{|c|c|c|c|c|c|c|}
\hline & Telescope & Instrument & $\begin{array}{l}\text { Weight } \\
(\mathrm{kg})\end{array}$ & $\begin{array}{l}\text { Size } \\
(\mathrm{m})\end{array}$ & $\begin{array}{l}\text { Data Rate } \\
\text { (bps) }\end{array}$ & $\begin{array}{l}\text { Average } \\
\text { Power } \\
\text { (W) }\end{array}$ \\
\hline \multirow[t]{3}{*}{ a. } & $\begin{array}{l}\text { Gregorjan } \\
\quad(>1200 \AA)\end{array}$ & $\begin{array}{l}\text { Universal filter } \\
\text { camera }\end{array}$ & 20 & $0.2 \times 0.2 \times 0.6$ & (or film) & 100 \\
\hline & & Video magnetograph & 20 & $1 \times 0.25 \times 0.2$ & $\begin{array}{l}3 \times 10^{5} \\
\quad(\text { or film) }\end{array}$ & 100 \\
\hline & & $\begin{array}{l}\text { High-resolution } \\
\text { spectrograph }\end{array}$ & 500 & $0.5 \times 0.5 \times 6$ & $\underset{\text { (or film) }}{12 \mathrm{M}}$ & so \\
\hline b. & $\begin{array}{l}\text { Normal incidence } \\
\text { tclescope } \\
(1600-300 \AA)\end{array}$ & $\begin{array}{l}\text { Spectrograph/ } \\
\text { spectroheliometex }\end{array}$ & 270 & $0.7 \times 0.6 \times 4$ & $100 \mathrm{~K}$ & 100 \\
\hline c. & $\begin{array}{l}\text { Grazing incidence } \\
\text { telescope } \\
(140-600 \AA)\end{array}$ & $\begin{array}{l}\text { Spectrometer/ } \\
\text { spectroheliograph }\end{array}$ & 150 & $0.2 \times 0.4 \times 2$ & ${ }^{i K}$ (or film) & 15 \\
\hline d. & $\begin{array}{l}\text { Crazing incidence } \\
\text { telescope } \\
(8-300 \AA)\end{array}$ & $\begin{array}{l}\text { High-resolution } \\
\text { spectrograph }\end{array}$ & 250 & $3 \times 0.5 \times 0.5$ & $20 K$ & 50 \\
\hline e. & & Filter camcrà & 50 & $0.5 \times 0.5 \times 1$ & ${ }_{\text {(or film) }}$ & 50 \\
\hline
\end{tabular}

¿Numbers do not include auxiliary equjpment such as electronics racks. computers, and monitors. The weight and power needed for these would be $-200 \mathrm{~kg}$ and $500 \mathrm{~W}$ continu ous, respectively. 
2. Representative Special-Purpose Instruments for a Large FinePointed Platform

\begin{tabular}{|c|c|c|c|c|c|}
\hline & Instrument & Size $(m)$ & $\begin{array}{l}\text { Weight } \\
\text { (kg) }\end{array}$ & $\begin{array}{l}\text { Data Kate } \\
\text { (bps) }\end{array}$ & $\begin{array}{l}\text { Average } \\
\text { Power } \\
\text { (W) }\end{array}$ \\
\hline a. & $\begin{array}{l}\text { Fxternally } \\
\text { occulted } \\
\text { coronagraph }\end{array}$ & $2 \times 0.5 \times 0.5^{a}$ & 200 & IOM or film & 50 \\
\hline b. & $\begin{array}{l}\text { Concave } \\
\text { grating } \\
\text { spectrohetiograph }\end{array}$ & $0.5 \times 0.5 \times 4$ & 200 & $20 \mathrm{~K}$ & 50 \\
\hline c. & Polarimeter & $0.5 \times 0.5 \times 4$ & 250 & 1000 & 50 \\
\hline
\end{tabular}

${ }^{a}$ Also requines occulting disk at $10-20 \mathrm{~m}$ distance.

\section{Representative Instruments for a Small Fine-Pointed Platform}

The following instruments will be of within a factor of about 2 of current rocket payloads in their weight, volume, power, and data requirements: uv high-resolution echelle spectrograph, matched double (normal and grazing incidence) spectrograph, monitor instrumentation in support of Solar Telescope Cluster instruments (e,g., H- $\alpha$ heliograph $\mathrm{x}$-ray spectrum monitor), developmental instruments (e.g., xuv magnetograph).

\section{Representative Coarse-Pointed, High-Energy Instrumentation}

\begin{tabular}{|c|c|c|c|c|}
\hline Instrument Type & $\begin{array}{l}\text { Mev Energy } \\
\text { Range }\end{array}$ & $\begin{array}{l}\text { Required } \\
\text { Pointing }\end{array}$ & $\begin{array}{l}\text { Primary Measure- } \\
\text { ment Objective }\end{array}$ & $\begin{array}{l}\text { Weight, Power, } \\
\text { Volume, Telemetry }\end{array}$ \\
\hline $\begin{array}{l}\text { Proportional counters } \\
\text { and/or cooled solid- } \\
\text { state detectors }\end{array}$ & $0.001-0.050$ & $\sim 5^{a}$ & $\begin{array}{l}\text { Spectral (atomic } \\
\text { line and continuum) }\end{array}$ & $\begin{array}{l}90 \mathrm{~kg}, 10 \mathrm{~W}, \\
0.13 \mathrm{~m}^{3}, 3 \mathrm{kbps}\end{array}$ \\
\hline $\begin{array}{l}\text { Actively shielded scin- } \\
\text { tjllators } \mathrm{Cs} \text { and } \mathrm{NaI}\end{array}$ & $0.030-0.600$ & $\sim 5^{\circ}$ & $\begin{array}{l}\text { Spectral (con- } \\
\text { tinuum) }\end{array}$ & $\begin{array}{l}450 \mathrm{~kg}, 20 \mathrm{~W} \\
1.0 \mathrm{~m}^{3}, 3 \mathrm{kbps}\end{array}$ \\
\hline $\begin{array}{l}\text { Actively shielded scin- } \\
\text { tillators CsI and Nal }\end{array}$ & $0.300-10$ & $\sim 5^{\circ}$ & $\begin{array}{l}\text { Spectral (con- } \\
\text { tinuam) }\end{array}$ & $\begin{array}{l}900 \mathrm{~kg}, 20 \mathrm{~W} \\
1.0 \mathrm{~m}^{3}, 1 \mathrm{kbps}\end{array}$ \\
\hline $\begin{array}{l}\text { Actively shielded cooled } \\
\text { solid-state device }\end{array}$ & $0.100-10$ & $\sim 5^{\circ}$ & $\begin{array}{l}\text { Spcetral (nuclear } \\
\text { line) }\end{array}$ & $\begin{array}{l}700 \mathrm{~kg}, 20 \mathrm{~W} \\
0.64 \mathrm{~m}^{3}, 4 \mathrm{kbps}\end{array}$ \\
\hline $\begin{array}{l}\mathrm{Lj} \text { and Be scattering } \\
\text { block polarimeters }\end{array}$ & $\begin{array}{l}0.001-0.005 \\
0.005-0.030 \\
0.030-0.200\end{array}$ & $1^{n}$ & $\begin{array}{l}\text { Continuum } \\
\text { pólarization }\end{array}$ & $\begin{array}{l}100 \mathrm{~kg}, 15 \mathrm{~W} \\
0.34 \mathrm{~m}^{3}, 3 \mathrm{kbps}\end{array}$ \\
\hline $\begin{array}{l}\text { Bragg reflection } \\
\text { erystal polarimeter/ } \\
\text { spectrometer }\end{array}$ & $0.001-0.010$ & $1^{\circ}$ & $\begin{array}{l}\text { Line and } \\
\text { continuum } \\
\text { polarization }\end{array}$ & $\begin{array}{l}40 \mathrm{~kg}, 35 \mathrm{w}, \\
0.13 \mathrm{~m}^{3}, 1 \mathrm{kbps}\end{array}$ \\
\hline $\begin{array}{l}\text { Neutron-sensitive } \\
\text { scintillators }\end{array}$ & $1-100$ & Coarse & Solar neutrons & $\begin{array}{l}230 \mathrm{~kg}, 25 \mathrm{~W} \\
0.8 \mathrm{~m}^{3}, 1.5 \mathrm{kbps}\end{array}$ \\
\hline
\end{tabular}

\section{Representative Instruments for a Large Fine-Pointed Platform}

The LFPP is required for flights of instruments of the following representative types-ATM instruments: balloon payloads, which are too large for the SFPP; large instruments under development for eventual use on the SRC or the freeflyer; arrays of medium-sized problem-oriented experiments. On sorties when the STC is not available, the LFPP may be used for flying large instruments such as the coronagraph and polarimeter. 


\section{8 \\ Life Sciences}

\section{I N T R O D U C T I O N}

The Shuttle era will provide the first opportunity to carry out a thorough experimental program in the life sciences in space under conditions approximating those of ground-based laboratories. The Skylab experience has already demonstrated the feasibility of performing many kinds of general experimental manipulations under weightless conditions and has provided considerable support for the concept of a manned space laboratory in which sophisticated biological and medical experiments can be done. The ability to manipulate experimental material directly rather than by automated remote control alone is essential in the life sciences, and the capability for immediate follow-up of new experimental findings is an important facet of any effective biological experimentation. Having these attributes the pressurized laboratory concept constitutes a major advance in capability for definitive life-sciences investigations in space. In addition to biomedical investigations relevant to man's well-being in space, basic principles of biology and medicine can be examined using the $0-g$ environment as a research tool. The laboratory will provide the operational conditions necessary for the evaluation of components of advanced life-support systems and of man-machine integration technology.

In the following sections are outlined examples of investigations in life sciences that are necessary to assure man's safety and to define further the conditions in which he can take part in spaceflight or that utilize the space environment to learn more of basic biological processes on earth. Shuttle capabilities and requirements in these studies are discussed. Studies relative to life elsewhere in the universe (exobiology) are not discussed because Shuttle capabilities here, other than as a launching or collecting platform, are minimal. 
Observations of the terrestrial environment (e.g., ecology, agriculture) are omitted as earth applications were excluded from the Study's charge. It is recognized that the scientific questions asked are based on 1973 concepts and technology and that maximum flexibility must be maintained for restructuring the questions and protocols with the development of new knowledge.

\section{I1. CELLULAR AND MOLECULAR BIOLOGY}

Theoretical and experimental grounds for predicting detectable effects of $0 \mathrm{~g}$ at the cellular, subcellular, and molecular levels are still fragmentary. The experiments of Biosatellite 2 and other early flights have given some clues to potentially significant effects of weightlessness at the cellular level, and early Shuttle flights should include well-designed and well-controlled experiments to confirm and consolidate potential problems identified by observations in these early experiments.

The weakness of the gravitational force relative to other physical and chemical forces operating over molecular dimensions suggests that $0 \mathrm{~g}$ is not likely to affect directly kinetic properties of biochemical reactions in vitro in a detectable or physiologically significant way. Similarly, the small size of procaryotic microorganisms would be expected to minimize potential gravitational effects. In any event, procaryotes cannot be used as models for eucaryotic systems, because of major differences between bacteria and higher forms in size, cellular organization, and mechanisms of fundamental processes such as cell division.

It seems more likely that gravity may significantly influence complex intraceliular or intercellular processes involving oriented supramolecular structures such as the mitotic apparatus. Indeed, both U.S. and Soviet studies have suggested a slight increase in random chromosome aberrations and mitotic abnormalities in response to spaceflight conditions. Significant perturbation of the process of cellular replication in the absence of gravity would be of clear importance to the ultimate understanding of fundamental mechanisms; it could also have serious consequences as a source of potential disturbance in normal processes of cellular proliferation and turnover during long-duration spaceflight. Studies of the kinetics of cell growth and cell division in plant and animal tissue culture and in rapidly proliferating tissues in vivo (e.g., bone marrow, skin, intestinal epithelium) will be required to assess quantitative and qualitative effects of the space environment and $0 \mathrm{~g}$ on cell 
replication and turnover time, including parameters such as chromosome replication, function of the mitotic apparatus, and cytokinesis. İt would be of interest to test responses to mitogens such as phytohemagglutinin as a probe for potential alterations in control of the division cycle. Wound repair involves another sort of proliferative system appropriate for study in $0 \mathrm{~g}$ : the rate of repair of a cored skin wound has been very carefully analyzed under conditions of normal gravity as has the repair of mechanical injury to bone marrow, and these systems could be used to study the kinetics of cell growth and division in $0 \mathrm{~g}$. Investigations of embryonic development under $0 g$ (see Section III on Organismic Biology) may also give information pertinent to the cell-division question.

An on-board centrifuge providing variable $g$ in the range of 0 to $1.5 \mathrm{~g}$ and capable of handling both tissue cultures and small animals of the size of mice or rats will be essential for these studies.

The Spacelab environment would not seem to offer any special opportunity for genetic studies other than those concerned with cell division and chromosome replication. The suggestion of synergism between radiation mutagenesis and weightlessness is probably neither a genetic nor a radiation problem but a problem in molecular or cellular reactions or both to $0 \mathrm{~g}$. It is not certain at this time that a better understanding of such $0 \mathrm{~g} g$ effects would have any importance for molecular or cellular genetics per se, but the possibility should be kept in mind for long-range planning.

It is not clear to what extent gravity may influence processes involving cellular and intracellular movement such as cytoplasmic streaming, rapid axonal flow, and amoeboid locomotion. Phasecontrast microscopy and photomicrography provide simple experimental means for investigating these questions.

The intriguing possibility of gravitational effects on membranemediated processes deserves careful consideration. At the present time there seems little reason to anticipate significant gravitational effects on carrier-mediated solute transport across biological membranes. Current information suggests that convectional forces are not important to the mechanism of biological membrane transport systems and that gravitational effects on the molecular organization of biological membranes should be negligible. For these reasons, investigation of possible effects of $0 \mathrm{~g}$ on the kinetics of carriermediated transport does not appear to offer a particularly fruitful area of study. However, study of electrolyte and water transport in model systems such as the toad bladder may be appropriate in 
relation to the problem of redistribution of fluid volume and electrolyte balance in man.

It is of interest to examine effects of weightlessness on phenomena involving specific cell-cell interactions, such as cell sorting in sponge and embryonic tissue and differentiation in the cellular slime mold. Density-dependent (contact) inhibition of growth in tissue culture would also be of interest.

\section{I I. O R G A N IS M I C B I O LOG Y}

In considering the potential effects of the space environment on the organism as a whole, a central scientific question is the long-term influence of weightlessness on growth, development, maturation, and reproduction. This question is conceptually the same for both plants and animals but for convenience is discussed sequentially below. Other major questions-effects on individual body systems of the mature organism and effects of radiation-are treated in Section IV on Biomedicine.

\section{A. Plant Biology}

The classic picture of the influence of gravity on plants is that of the positive and negative geotropism exhibited by stem and root tissue. Obvious questions have arisen as to what is the receptor mechanism by which plants perceive gravity and what are the mechanism(s) that mediate the tropic response. Although numerous ground-based experiments have been performed, no single hypothesis has been formulated that explains or clarifies the phenomenon to the satisfaction of a majority of scientists. Studies carried out on Biosatellite 2, although providing rather uncertain results, indicated that ground-based control experiments employing the clinostat gave results comparable with those carried out at $0 \mathrm{~g}$. We agree in general with the consensus of earlier study groups that future experimentation should be largely limited to precise ground-based experiments aided by clinostat studies to mimic weightlessness. However, it should be emphasized that the validity of the clinostat as an adequate model of $0-g$ conditions for plant studies must be unequivocally demonstrated. A variable-g centrifuge in the pressurized laboratory would strongly aid in this evaluation.

Geotropic experiments should be performed only as part of experiments designed to examine other aspects of plant and cell 
development that are thought to be influenced by gravity. Only those experiments that have had the most thorough exploration under $1-g$ conditions should be considered for analysis at $0 \mathrm{~g}$. As part of this evaluation, it is important that thorough analysis and review of any potential flight experiment by a good cross section of the concerned section of the scientific community be obtained.

Equal attention should be directed toward growth and development of plant cells in $0 \mathrm{~g}$. For example, does the absence of gravity influence the morphogenesis of plant cells and affect chromosome replication to alter the mechanism of cell division? Previous flight studies have indicated that chromosomal aberrations do occur, and clinostat data suggest alteration of certain enzyme activity (glutamine synthetase) and of tracheid formation. Additional information as to the cause of such effects might contribute to a more tenable explanation for the ability of plants to respond to a gravitational field.

In preparation for Shuttle experiments, a thorough study should be made of the types of plants that would provide the most useful information concerning the wide range of phenomena expressed by plants as a function of gravitational field.

Many of the experiments proposed for $0-g$ conditions encountered in spaceflight are directed toward short-term responses of organisms because of the limited duration of available flights. Although results from short-term $0-g$ experiments will augment thinking about possible effects produced by longer exposure, the obvious question, derived from extrapolation of the possible effects of weightlessness on chromosome replications, is whether plants can grow and develop normally in long-duration spaceflight. For example, can a microalgae population continue in a normal fashion after several generations at $0 \mathrm{~g}$ ? Such information would be essential if one were to propose plant systems as secondary life-support systems in long-duration spaceflights. If malfunction of cell division is induced by extended $0 \mathrm{~g}$, populations of microalgae and higher plants would diminish, photosynthetic capacity decrease, and the support systems ultimately fail. Long-duration growth and development experiments might also provide important evidence for the possible evolutionary mechanisms of terrestrial plants. Plants that are able to complete one or more life cycles within 30 days, such as Aribidopsis, or that are easily grown in tissue culture (carrot, tobacco) or that produce extensive xylem proliferation (sunflower) would be typical objects for experimentation. 


\section{B. Animal Biology}

Thirty-day Shuttle flights and recoverable free-flier flights of six months or more provide an excellent opportunity for long-term $0-g$ experiments with animal systems. Results of complete life-cycle studies on small mammals such as mice and rats should provide important information, transferable to man in many cases, on physiological effects of weightlessness.

A unique feature of the $0-g$ conditions in the pressurized laboratory will be the opportunity to determine in aqueous medium the morphogenic characteristics of some simple metazoans (hydra or perhaps copepods) and to make further studies of protozoa. The purpose of this type of experiment would be to evaluate whether the earth's aquatic and marine environments are truly $0 \mathrm{~g}$ in their morphogenic effects. Similar studies on the fine structure of various diatoms would represent an alternative way to examine this interesting aspect of possible gravitational effects on the development of aquatic organisms.

The placental mammal in utero, like aquatic animals, might be considered as developing in a generally omnidirectional force field. If so, then gestation in a weightless environment should have little or no effect on the developmental processes of organogenesis and fetal growth. However, the suggestion is speculative and should be tested. For example, do blastocyst formation and implantation have any dependence on external force fields? To what extent might critical delineations of organ systems be thus dependent? Is parturition at all gravity-dependent? Postnatal growth and development in $0 \mathrm{~g}$ should then identify the adaptive sequence to which we are committed by our terrestrial confinement. For example, if cardiac "deconditioning" is solely a function of $0 \mathrm{~g}$, then a mammal brought from birth to young adulthood ( $\sim 25-30$ days for the mouse) in the absence of gravity might be irreparably "deconditioned." Aspects of mineral metabolism and concurrent musculoskeletal development might be clarified by studies of early postnatal growth in $0 \mathrm{~g}$.

To carry out these studies, animal-holding facilities are required for small mammals and selected avian species. An on-board centrifuge with a range of 0.1 to $1.5 \mathrm{~g}$ should be available for the smaller species to define effects of fractional $g$. Dissection and preliminary fixation of tissue samples would be required. Mission duration should be no less in duration than the period from fertilization through parturition of the animals in question; and a polar orbit, with concomitantly higher levels of radiation, should be avoided. 
Study of the behavioral patterns of lower vertebrates, perhaps small fish such as goldfish or guppies when exposed to $0 \mathrm{~g}$ in an aquatic environment, would provide additional information on the ability of such organisms to maintain themselves in the appropriate pressure/buoyancy gradient. This type of study would give insight into the mechanism of function of the swim bladder and possible other position-orienting sensors in aquatic vertebrates. A preliminary demonstration on Skylab 3 using minnows indicated that fish brought into space adapted fairly quickly and that those born in space were adapted to $0 g$ from birth. Whether they can subsequently survive under $1 \mathrm{~g}$ is not yet clear.

Rhythmicity in biological phenomena has fundamental importance in all life-sciences disciplines. Processes that are cyclic in their time response (circadian rhythm) are found over a broad range of responses, i.e., from nucleic acid synthesis to complicated stress reactions in plants, animals, and birds, and have had extensive study in biological systems on earth. This topic was covered in depth by the Santa Cruz study in 1969,* and some of the experiments proposed there were flown on Skylab but failed because of electrical malfunction. While they should be studied under the unique circumstances provided by spaceflight, several circadian rhythms have been shown in ground-based experiments to be genetically determined and genetically modifiable rather than governed by external processes. $\dagger$ This renders experiments on circadian rhythms in actual spaceflight less critical. Barring observations on the ground or in space that override the work of Konopka and Benzer, a lower priority for this type of biological experimentation is recommended.

\section{B I O M E D I C I N E}

The space environment offers new experimental approaches to analysis of mechanisms of a variety of fundamental physiological control systems. In addition, certain human (and mammalian) functions have become deranged in space, and corrective or preventive measures must be sought in order that man may perform adequately. Other functions offer special advantages for investigation of the $0-g$ condition.

*Space Science Board, Space Biology (NAS, Washington, D.C., 1970), Chap. 2. $\nmid$ R. J. Konopka and S. Benzer, Proc. Nat. Acad. Sci. (U.S.), 68, 2112 (1971). 


\section{A. Cardiovascular System}

Prolonged spaceflight is accompanied by complex physiological changes, and evidence to date suggests that these changes may be manifest most in the cardiovascular system. Disturbances that can be related to the cardiovascular system have consistently occurred in astronauts and cosmonauts and have been primarily evident as a decreased tolerance to the burden of gravity after re-entry to earth. The regulatory pathways that normally maintain arterial blood pressure and peripheral blood flow on earth necessarily are still present at $0 \mathrm{~g}$, and some alteration has apparently taken place, rendering compensation for $1 \mathrm{~g}$ less effective.

The essential responses of heart rate, strength of contraction of the heart muscle, and peripheral vascular resistance are the result of complex reflex interactions among (a) the mechanoreceptors in the heart, lungs, aortic arch, and carotid sinus; (b) the central nervous system and autonomic nerve outflow; and (c) the smooth muscle of the blood vessels and the heart, all reacting to maintain blood flow to the vital organs. Experiments in both animals and humans will be needed to determine which parameters must be monitored inflight to detect functional changes in the cardiovascular control system and what procedures are effective in combating or preventing these changes. Examples of pertinent experiments that could be carried out in the pressurized laboratory are measurement of peripheral blood flow by venous occlusion plethysmograph during lower-body negative pressure (LBNP) or during a deep breath and determination if exercise, isometric exercise, or LBNP prevent the abnormal cardiovascular regulatory responses that are seen after exposure to weightlessness.

It is presumed (see Section IV. C on Kidney and Metabolism) that substantial loss of sodium and water occurs on entry into $0 \mathrm{~g}$. If so, it may be initiated by the movement of extravascular fluid into the capillaries of the lower body under osmotic forces. Experiments are needed to determine the validity of this hypothesis, particularly in relation to the long-term changes that can be expected.

There are reasons to expect that the pressure and volume workload on the right heart might be reduced in space, leading to some decreased efficiency. This could be critical on return to the $1-g$ environment after prolonged residence at $0 \mathrm{~g}$. Experiments should be designed to study possible atrophy of cardiac and other muscles. 


\section{B. Respiration}

There are no apparent reasons to expcct any significant alterations in cellular respiration, gas diffusion exchange, or control of respiration as a result of $0 \mathrm{~g}$ except for changes secondary to any different total body energy requirements.

As the atmosphere of the orbiter cabin and pressurized laboratory will be air at sea-level pressure (20\% oxygen, $80 \%$ nitrogen at $760 \mathrm{~mm} \mathrm{Hg}$ ), with the partial pressure of carbon dioxide maintained at less than $7 \mathrm{~mm} \mathrm{Hg}$, the basic composition of the gas that the crew and passengers will inspire presents no difficulty. However, as discussed in Section VII on Life Support, the atmosphere must be monitored assiduously and excess gases and particulate matter removed.

The absence of gravity will alter the mechanical function of the lungs. Lung function should be studied after a protracted stay in space to determine if there is significant deterioration of muscles and supporting structures. The lack of hydrostatic pressure in the pulmonary circulation may alter the distribution of capillary blood flow/alveolar ventilation through the lungs and decrease the effective pulmonary vascular resistance. Spaceflight provides a unique opportunity to study these effects. Ciliary transport, lymphatic drainage, and phagocytic functions may become abnormal after exposure to $0 \mathrm{~g}$. These mechanisms are of great importance in protecting the lung from infection and are deserving of study.

\section{Kidney and Metabolism}

It is our present hypothesis that, with the entry of man into a weightless environment, there occurs a rapid translocation of interstitial fluid into the intravascular space with an attendant activation of volume control mechanisms leading to an increased rate of renal excretion of sodium, chloride, and water. With the net contraction of extracellular fluid volume, there may follow an increase in the rate of aldosterone secretion leading to an increase in the renal excretion of potassium. If the excretion of potassium exceeds the concurrent rate of intake, total body potassium depletion will ensue. The latter could have widespread physiological effects on the cardiovascular system (as may have occurred in Apollo 15) and on the rate of secretion and effects of certain hormones such as insulin and growth hormone. The perturbation of volume regulation imposed by the weightless state offers the opportunity to 
examine the component parts of this complex control system in a manner that is not possible on earth.

The astronauts have shown a negative calcium balance in space, presumably analogous to that seen in prolonged bedrest at $1 \mathrm{~g}$. Although the calcium loss has not been accompanied by signs of general demineralization of the bones, it is important to determine if it will continue for as long as man is at $0 \stackrel{g}{g}$ or whether it levels off or can be made to do so. It may be possible to obtain information about calcium metabolism as well as about any perturbations in the rate of bone formation in $0 \mathrm{~g}$ by monitoring the repair of minute mechanical injuries made to the bone marrow of small animals. The rate of repair on earth has been very carefully timed. It provides a useful model because it involves the deposition and resorption of cancellus bone.

The absence of gravitational force may reduce the total basal energy requirements of the body, which would produce a lower metabolic rate, or qualitatively altered metabolism, detectable for example as a decreased food intake or lower body temperature.

\section{Hematology}

There has been a consistent decrease in red-blood-cell mass in the astronauts produced by increased destruction of red blood cells as well as a suppression of the bone marrow's normal compensatory response. It has been generally assumed that the basic cause was the hyperoxic atmosphere breathed by the astronauts, because ground studies in similar atmospheres were able to duplicate the effect. However, an anemia appeared in the first two Skylab missions despite a normal inspired $\mathrm{O}_{2}$ tension, suggesting the influence of other factors. It is important to uncover the cause of this decrease in red-cell mass and to determine whether it plateaus or progresses with the stay in space. Studies should be done to learn if the rate of turnover is changed and also to learn the rate of granulocytic response to inflammatory stimuli.

\section{E. Neurology}

It was anticipated that $0 \mathrm{~g}$ would affect the vestibular apparatus. Astronauts have indeed had some difficulty with motion sickness, but except in Skylab 3 it has been relatively transient; susceptibility is not predictable from ground-based tests. Recent results from Skylab 2 and 3 indicate that once adaptation has taken place the 
otolith organ is functionally denervated in $0 \mathrm{~g}$ because moving the head forward and sideways while rotating in a chair (a maneuver that leads to symptoms of motion illness at $1 \mathrm{~g}$ ) produced no effect. Advantage should be taken of the 0.g environment to study vestibular functions in man and animals.

Other neurophysiological dysfunctions may appear, secondary to the changed relationships between the antigravity and other muscle groups or to the altered afferent input to the cardiovascular system. Animals raised from conception in $0-g$ environment might show important neurophysiological defects at $1 \mathrm{~g}$, because development of the nervous system is critically dependent on the presence of the relevant stimuli at certain stages.

\section{F. Microbiology}

Microbiological problems in spaceflight divide themselves into two major categories: those concerned with interactions of microbial flora within an enclosed system, including possible alterations in host resistance, and problems arising from the possibility of mutation during flight leading to the emergence of additional pathogenic microorganisms.

The possibilities of mutation leading to new pathogens have been explored, and, although this must always be considered, the risks do not seem much greater than they would be on earth. It seems likely that alterations in host resistance attendant upon prolonged spaceflight and the spread of microorganisms within an enclosed space will be more important considerations.

Although there is a tendency to equate host resistance with antibody formation, function of phagocytes, and activity of immunocytes, it is often forgotten that the first lines of defense consist of relatively nonspecific teactions, such as the intact integument, rinsing mechanisms such as tears and other secretions, the motility of the gut, the extremely active capacity of the alveolar macrophage system to kill inhaled bacteria in aerosols, and the antibacterial mechanisms in mucous membranes. It is still not clear whether $0 \mathrm{~g}$ will have a deleterious effect on some of these defenses, such as rinsing mechanisms or the capacity of the lungs to clear inhaled bacteria because of altered deposition of aerosols in the lungs. A major potential problem is gastrointestinal disturbance. It is known that the motility of the gastrointestinal tract is a major factor in keeping the duodenum and jejunum relatively free of bacteria. The degree to which such motility and the movement of bacteria will be 
influenced by $0 \mathrm{~g}$ remains unexplored. There is increasing evidence that prevention of and recovery from disease are dependent on cell-cell interactions and cellular immunity. Study of the effect of $0 \mathrm{~g}$ on these cellular functions relative to host resistance is thus very important.

Specific immune responses to spaceflight have been monitored in previous flights and do not seem to have been greatly disturbed. However, we must remain alert to this possibility.

Preflight isolation of astronauts and immunization procedures have reduced almost to zero the incidence of inflight illnesses, and it is mandatory that precautions of this nature be continued in Shuttle flights. They will require continuing review and adjustment in accordance with changing knowledge and increasing experience. Epidemiological study of the environments of the flight personnel and their families during the preflight period would also appear to be essential. Conditions favoring the exchange of microorganisms among individuals are not yet well understood on earth let alone in space, and further work is needed so that control measures can be fashioned if such exchange proves harmful. The life-support systems should remove viable microorganisms from the Shuttle atmosphere or kill them as the most practical way of reducing this potential hazard. Finally, the possibility of replacing the usual earth flora in the microbial environment with relatively innocuous organisms should be given much more attention than it has received so far.

\section{B E H A V I O R}

Although much indirect evidence has been accumulated about man's ability to perform in spaceflight, there is a need to quantify man's performance in order to plan properly for his effective utilization in all modes of spaceflight activity. Most of the planned Shuttle missions have tasks that might be used to investigate and quantify the human operator's visual, mental, and psychomotor performance and levels of stress, boredom, or fatigue. The development of precise tests that are able to discern subtle decrements in performance, and perhaps changes in behavior, will be necessary in preparation for longer flights when the ability to predict decrements will be important. Study of existing tapes of communications during flight should yield detailed observations on the interchanges among personnel and permit the study of interpersonal interactions and relationships. The data should identify reaction patterns between 
colleagues under these unique conditions and perhaps suggest improved personnel screening techniques.

Preliminary findings indicate that quality of sleep is not significantly altered in spaceflgiht, whereas there may be an alteration in sleep quantity. Regardless of what may be learned concerning sleep during Skylab missions, the question of mental state, i.e., alertness, and performance in relation to sleep, will not have been answered. The Shuttle flights provide an excellent opportunity to investigate this relationship.

The participation of a scientist-passenger population with professional astronauts in the Shuttle flights will require modification of selection criteria and screening procedures and, finally, assessment of the techniques for evaluating the performance of those individuals ultimately selected. The results of such investigations would serve the dual purpose of validating the selection criteria and providing insight for improving subsequent screening methods for future participants in spaceflight missions. In addition, studies should be made to determine how much and what kinds of training are necessary to enable the scientist-passenger to tolerate and perform well under the unusual environmental conditions in space.

\section{RA D IO B I O LO GY}

The radiations encountered in spaceflight can present a hazard to man during long-duration flights where flight trajectories forbid quick return. There are no unique radiations in space, with the exception of the heavy-ion or HZE-particle component. Thus, present understanding of radiation effects is quite adequate to permit accurate prediction of the consequences of space-radiation exposure and to prepare countermeasures as needed or appropriate.

There are two areas of possible exception: the biological effects of HZE-particle irradiation and the possibility of synergisms between radiation and other elements of the space environment. The first is a matter of ascertaining if randomly located microlesions, induced by HZE particles being stopped in deep tissue, might cause sufficient cumulative damage to nonproliferating cells that pathological consequences might ultimately result. Could HzE-particle hits, for example, induce a detectabie loss of retinal function?

For both scientific and practical reasons HZE-particle effects are best studied initially in ground-based laboratories. The studies required and the methods of study are described in detail in a 
recently issued Space Science Board report.* One strong recommendation has been made in that and other related reports: basic studies on radiation effects should not be attempted in flight laboratories.

Ground-based studies with accelerator-produced heavy ions are thus the first requirement. NASA has the operational problem of assessing the potential hazard of HZE particles to man during longduration space missions and should therefore take full advantage of the ground-based facilities that are becoming available. Flight studies can then be designed for proof-of-principle.

If flight experiments on HZE-particle effects do develop sufficient priority as a result of the earth-based work, then a polar orbit should be sought. With minimal shielding, about 3 iron nuclei might be stopped per day in each gram of tissue. At lower orbital inclinations, the geomagnetic cutoff would reduce the expected number of hits by a factor of 10 or more.

The exposure of biological specimens to the ambient flux of heavy particles should not be encouraged on the assumption that the HZEparticle microlesions will be readily observable in deep tissue and their pathologic consequences then identified. In fact, it is forbiddingly difficult to detect such lesions because of their minute size and random location. All significant HZE events (thindowns or stopped particles) impinging on the biological target must be recorded and geometrically correlated with the target so that the precise location of the microlesion can be anticipated. The Biostack and Biocore experiments of the Apollo program are examples of two methods for correlating physical and biological events. Additional techniques should be sought, especially for lesions in deep tissue.

The question of synergism between radiation injury and weightlessness, or other flight-related factors, largely raised by some of the uncertain results of the Biosatellite 2 experiments, cannot logically be resolved by immediately embarking upon a series of flight experiments. There seems to be evidence accruing from both U.S. and Soviet studies that $0 \mathrm{~g}$ has an impact on cell division. The evidence is seen in the form of small increases in certain chromosome aberrations, spindle misorientation, and cell lethality (of chromosomal origin?), but the mechanism that might produce these findings is not clear. The molecular basis of $0-g$ perturbations of cell division

* Radiobiological Advisory Panel, SSB Committee on Space Biclogy and Medicine, HZE-Particle Effects in Manned Spaceflight (National Academy of Sciences, Washingtcon, D.C., 1973). 
should first be thoroughly studied (see Section II on Cellular and Molecular Biology) before studies of synergism are undertaken. Some critical studies might be possible on molecular aspects of radiation target theory and the many associated concepts of genetic and cellular radiation injury. These, too, must be preceded by more complete evaluation of strictly $0-g$ effects on cell metabolism and reproduction.

Ultimately, sophisticated multivariate radiobiological experiments for flight could be conceived. These would require on-board radiation sources, both low- and high-LET, the capacity for in vitro and in vivo cell labeling with tritiated and carbon-14 labeled compounds, and the use of both cell cultures and small mammals. While such studies would best be done in orbits of about $30^{\circ}$ inclination to avoid the cosmic radiations as much as possible, studies on heavy-ion effects, as noted above, will require a polar orbit. The latter, however, may also best be designed not to require any complex inflight manipulation but to allow a maximum buildup of particle hits for later evaluation on the ground.

All Shuttle flights should contain dosimetric devices and materials positioned to accumulate data on the flux and energy of the several radiations in space and the shielding effect of spacecraft components on their intensity and scattering. While the influence of these components on the actual radiation levels in different parts of the spacecraft can be predicted to some degree, it is necessary to test these expectations against observations.

\section{LIFE-S UPPORT TE CHNOLOGY}

Requirements for atmospheric control in spacecraft are (a) to supply oxygen in the amounts needed to sustain life; (b) to maintain appropriate temperature, pressure, and relative humidity; (c) to remove carbon dioxide and water produced by metabolism; and (d) to monitor and remove trace contaminants and particulate matter.

With the present state of the art, for the short-duration missions presently planned for the Shuttle, open-loop systems, in which oxygen is supplied and wastes are removed and stored, weigh less and use less energy than closed-loop systems in which wastes are recycled. From the standpoint of weight and power requirements, the present breakeven point between open-loop and closed-loop systems is about 30 days. 
If development of recylcing systems proceeds, there is a high probability that in a very few years such systems will be able to compete with open-loop systems for missions of two weeks or even less. A wide variety of systems have been proposed for the recovery of oxygen from both carbon dioxide and water under the conditions prevailing in space. Examples of such systems include chemical conversion (e.g., Bosch, Sabatier), electrochemical (e.g., fused carbonate, solid electrolyte), electrolysis cells, and bioregenerative systems. None of these systems is yet at the point of acceptability in space from the standpoint of weight, energy requirement, or reliability, although substantial progress has been made.

It has been suggested that the lack of gravitational force would affect transport through artificial membranes, an important process in many proposed life-support systems. The rate of transport of a molecular species across a membrane system can be dependent on gravity only if gravitational forces affect concentration gradients in the system. This might occur, for example, if gravity-dependent convection is required to prevent the development of concentration gradients in the boundary layer adjacent to the membrane. However, it is presently possible, within the current state of the art, to design out any adverse effects caused by $0 \mathrm{~g}$. Forced convection and the use of capillary action in porous structures are presently utilized to minimize adverse $0-g$ effects on ionic and nonionic solute rejection, concentration gradients at the membrane surface, absorption phenomena, etc., in such devices as batteries and fuel cells designed for operation at $0 \mathrm{~g}$.

The orbiter cabin and pressurized laboratory should provide facilities for the testing of components of closed-loop life-support systems developed on the ground on a competing basis, so that efficient and reliable systems can be developed. In particular, appropriate space, power, and access to the main atmospheric circulation loop should be provided for at least two prototype component units to be tested simultaneously.

The closed environment of the Shuttle will undoubtedly produce a wide variety of trace contaminants, some of which will be dangerous or distressful. Many of these contaminants have synergistic effects on body function. For example, trace amounts of ozone in the atmosphere produce lung tissue damage and also have a profound effect on the ability of the lungs to clear microorganisms. Man and other animals also produce a variety of chemicals that are eliminated into the ambient in trace amounts. The rates of their buildup in a closed 
system have not been studied adequately, nor have their possible deleterious effects on man, animals, or plants. Plants, for example, are exquisitely sensitive to ethylene. Apparently the only contaminant-monitoring systems currently being contemplated for the Shuttle are a rudimentary carbon monoxide sensor and, possibly, a hydrogen leak detector. The only contaminant-scavenging systems now being planned include the use of $\mathrm{LiOH}$ for carbon dioxide removal, filters for particulate matter, and activated carbon for removal of material for which it has an affinity, such as highmolecular-weight matter. Clearly, a better system to remove trace contaminants will be required, such as a catalytic burner or a rechargeable absorbing system. It should be pointed out that rather sophisticated systems for sensing and scavenging contaminants are curtently available and in commercial use. Both for crew health and safety and for the success of scientific experiments, a substantial improvement in the contaminant control system over what is presently contemplated will be necessary.

For safety reasons it is mandatory that the air in the orbiter cabin be monitored continuously for oxygen and hydrogen, the first to guard against a significant reduction in oxygen for breathing and the second, as an index of a fuel leak, to prevent fire. In addition, it is most important that the atmosphere be monitored periodically (several times a day) for trace contaminants such as carbon monoxide, oxone, amines, sulfides, mercaptans, and hydrocarbons. The particulate content of the cabin air must also be sampled and measured periodically and the number of viable microorganisms determined. There will need to be a mechanism for removing this particulate matter, as by filters, and possibly for sterilizing the air stream, as by ultraviolet radiation. Trace contaminants and particulate matter, including microorganisms, must be removed to levels consistent with standards recommended in reports of the NRC Committee on Toxicology* and the Space Science Board. $\dagger$

Recent developments in the computer sciences indicate that, by the 1980's, Shuttle users can expect that their needs for inflight

\footnotetext{
* Panel on Air Quality in Manned Spacecraft, NRC Committee on Toxicology, Atmospheric Contaminants in Space craft (available from NASA Director of Life Sciences, Washington, D.C.).

†SSB Panel on Air Standards for Manned Space Flight, Atmospheric Contaminants in Spacecraft (NAS-NRC, Washington, D.C., 1968); Physiology in the Space Environment, Vol. II, Respiration (NAS-NRC, Washington, D.C., 1967); Infectious Disease in Manned Spaceflight: Probabilities and Countermeasures (NAS, Washington, D.C., 1970).
} 
computation will not be limited by size and weight considerations. One important potential use for this improved on-board computer capability will be in the teleoperator, or remote manipulator, system. Projected developments in teleoperators, particularly improved hand geometry and use of tactile- and force-feedback, make it probable that many of the presently contemplated needs for extravehicular activity in the Shuttle-Spacelab can be handled remotely. Accordingly, new and improved designs of teleoperators should be field-tested in the Shuttle-Spacelab to determine their suitability for general use in space, and facilities for such field-testing should be provided.

\section{LABORATORY OPERATIONS}

The wide range of experimental approaches employed by the various biological and medical disciplines will require a pressurized laboratory module of the maximum size possible and with maximum flexibility as a major design principle. Requirements for specific experiments will vary greatly among different investigators and from flight to flight. However, certain common requirements can be identified; these include light, water, electricity, thermal control within $\pm 2{ }^{\circ} \mathrm{F}$, suction, laboratory refuse disposal (solid, liquid, gaseous), and such generalized equipment as a small multipurpose centrifuge, refrigerator, and freezer. It is impossible to identify or enumerate all types of specialized instrumentation required for individual missions, but it is likely that major classes of equipment used in many types of investigation would include, for example, radioisotope-handling apparatus, constant-temperature incubators, plant-growth and tissue-culture facilities, aquaria, photographic instrumentation, microscopes, and animal-holding and -handling facilities. We do not anticipate special requirements or problems with respect to data handling and storage. However, need for an on-board centrifuge to provide a control for the gravity component and to test responses to fractional $g$ forces has been emphasized by several disciplines.

The centrifuge should be a variable-speed device capable of generating accelerations up to $1.5 \mathrm{~g}$ and handling tissue cultures, plants, and small animals up to $0.5 \mathrm{~kg}$. Because animals in the centrifuge would be exposed to variations in acceleration and to Coriolis forces, which could produce physiological effects invalidating their use as l-g controls, the centrifuge should have as large a radius as possible to reduce the necessary speed of rotation and 
therewith the acceleration gradients. Rodents (white mice) have a normal metabolic rate during chronic exposure to between 1 and $2 g$ in a 6-ft-radius centrifuge, suggesting relatively normal behavior.* However, there is little similar information available on the behavior of higher vertebrates, especially primates, and such data should be obtained. The increased resistance of Skylab astronauts to Coriolis sickness may indicate that under $0-g$ conditions centrifuged animals will be less susceptible to vestibular-type dysfunctions in a centrifuge. The speed of rotation of the centrifuge should be regulated so that variations in $g$ force are minimal. Data are lacking on how large a variation is acceptable; intuitively we estimate that the variation should be less than $\pm 2 \%$, but this should be tested. Certainly the radius should not be less than $5 \mathrm{ft}$. The addition and removal of animals or biological materials to and from the centrifuge should be possible without stopping or starting the centrifuge. The capability of $0.5 \mathrm{~kg}$ will permit study of a small primate-a spider monkeywhich is important for man-related experiments. The centrifuge will not be required for all experiments and should therefore be removable from the laboratory module in order to conserve weight and space.

The above, very incomplete enumeration implies two important points. The first is that there can be no such thing as the ideal space laboratory for all biomedical work: there are too many different sets of equipments that will be needed at one time and not another. The design of the pressurized laboratory should therefore be based on modular concepts and interchangeable components. This will furthermore reduce lead times necessary for experimental work as the investigator will be responsible for furnishing his specialized equipment that can be plugged into the basic laboratory facility. The second point is the need to assure, insofar as possible, that the basic laboratory facilities (power and atmosphere control, for example) will in fact accommodate the requirements of the majority of potential users. This will require further contract study.

A prime requirement of the life-sciences laboratory is the ability to carry out investigations on the intact animal. The exact species that might be employed cannot be set out other than to identify the familiar vertebrates used in experimental biology and medicine. Among the mammals this would include mice, rats, Chinese

*W. Fethke, K. M. Cook, S. M. Porter, and C. C. Wunder, "Oxygen Metabolism Measurements during Chronic Centrifugation of Mice," J. Appl. Physiol. 35:572, 1973. 
hamsters, rabbits, and possibly dogs and small primates. Among the nonmammalian species, needs might arise for avian species up to the size of chickens and fish, frogs, and diverse amphibia and reptiles.

Adequate atmospheric and thermal control of the animal-holding facilities is essential. Ambient temperature should be held to about $72 \pm 2{ }^{\circ} \mathrm{F}$ and about 40 to $60 \%$ relative humidity for most mammals and birds. Lower or higher temperatures may be required for some reptiles, amphibia, and fish. We would prefer that a separate air input system be provided for the animal facility in order to avoid mixing of the two atmospheres except when animal handling is required, but there is no reason why the two atmospheres should not vent into a common exhaust and revitalization system. Total isolation defeats the purpose of the facility: full access to the animals must be available throughout flight. In addition to simple access, provision should also be made for some rather detailed manipulations including dissection and removal of selected organs and tissues. An especially difficult problem might arise when the gastrointestinal tract will have to be sampled, as may well be required in certain studies on cell proliferation. As microorganisms comprise the major component of intestinal contents, these contents as well as other body fluids, hair, and dander will have to be safely contained without inhibiting the investigator's manual or instrumental access.

Additional presently foreseeable requirements involving animals are the capability for radiologically safe use of radioisotope-labeled compounds for injection and on-board tissue or fluid sampling, counting, and disposal; artificial insemination of small mammals; small mammal nesting, parturition, and litter-rearing; avian egg incubation; and sampling of tissues and fluids and their storage for postflight chemical analysis. Small, live-in compartments may also be needed on the centrifuge for those species upon which the critical tests of weightlessness are made.

While certain of the projected experiments in the life sciences can be accomplished within the span of 7-day missions, others, such as those concerned with embryonic and fetal development, wound healing, and other aspects of cellular proliferation (e.g., marrow, skin, and gut), will require the full capability of the 30-day mission. In general, we anticipate that the longer missions will be of particular value to the life sciences. In some cases, free-flying unmanned satellites, recoverable after 6 months or so, will be necessary. For most purposes, orbital attitude and inclination will not be critical. Polar orbits may be required occasionally for radiobiological experiments. 


\section{X. EXPERIMENTAL AND ADMINISTRATIVE APPROACHES}

If the potential of the Shuttle for life-sciences research is to be realized to any meaningful extent, certain experimental and administrative approaches are essential.

All persons on all missions should be available for routine biomedical tests and monitoring. The critical need for information about the responses of humans to $0 g$, necessary to assure the safety of manned flight, means it would be wasteful not to obtain physiological data on all crew members and other personnel in addition to any life-sciences experimenters. Many of the Shuttle flights will not have the pressurized laboratory or life scientists on board, so the types of measurements made would necessarily be relatively unsophisticated. For example, at this time there is an identifiable need for metabolic balance studies, requiring collection and preservation of urine and fecal samples. This demands that the feeding and waste management systems of the orbiter be designed to permit such tests and that space be allotted for the samples obtained. Measurement protocols should be designed not to interfere with mission tasks.

The degree to which nonastronaut scientists of all disciplines are able to participate in Shuttle flights will have a strong impact on the amount of research that can be done in the Shuttle. Medical and psychological screening should therefore be as lenient as possible, within safety limitations. The initial period of conservatism in selection should be as short as possible, its end hastened by continuing tests to warrant lowering of criteria.

As a general principle, biomedical experiments should be conducted in humans first, where feasible, and supplemented by animal studies. Experiments should not overload the test subjects by trying to obtain many different kinds of data in one protocol: early experiments at least are likely to be more successful if they are quite simple in design and execution.

A cardinal rule is that all flight experiments should be preceded by adequate and thorough ground-based preparation. The responses of the test materials must be completely familiar under $1 \mathrm{~g}$ and the other experimental conditions. In no other way can the quality of the experiment and the legitimacy of any flight results be assured. The above implies that financial support for ground-based preparatory work will be necessary in some cases. Such support is justified, but it must not be abuised or allocated without careful review. 
Peer review of the entire structure of space life-sciences programs, both supporting research and technology ( SR\&T) and flight experiments, both proposed and on-going, is essential. The reviews should be made at regular intervals, systematically, by formal panels appointed for fixed terms and consisting of members of the national and international scientific community. This procedure should be initiated promptly, because out of the current SR\&T programs will doubtless come many of the Shuttle flight experiments.

It is imperative that the base of life scientists participating in space research be broadened. Similarly, communication between life scientists and the space program must be improved. Broad dissemination of information on flight opportunities should be helpful, as should appointment of a wider circle of scientists to review panels. Nevertheless, the most important influence to bring about a lasting, satisfactory participation of the scientific community is the continuing execution of high-quality work.

At present there is no formal representation for the life sciences within the administrative structure of ESRO. If the European community proceeds with the space laboratory, this lack will almost inevitably have an adverse impact on the quality of the biomedical laboratory. Appointment of staff and panels, as appropriate, would seem essential to this work and to increasing the participation of European life scientists in the Shuttle program.

\section{R E COMMENDATI ONS}

1. For life-sciences work in space, within the Shuttle concept, a pressurized biomedical laboratory that is as large as feasible is necessary. The laboratory will be used for a broad array of biological and medical experiments and must have flexibility as an intrinsic characteristic of design. We can identify some general requirements that will have to be built in, for example, that about 3 to $4 \mathrm{~kW}$, or about $3000 \mathrm{kWh}$, of power will be needed for a 30-day mission. In addition, many special requirements will be dictated by specific experiments that will differ from flight to flight. Thus modularity of equipment and facilities is important to permit interchange within common spatial dimensions and consequent saving of weight and space.

2. We foresee a requirement for recoverable unmanned free-flying satellites, or bioresearch modules, for experiments requiring long periods ( $\sim 6$ months) in orbit. A typical example would be the 
long-term effects of weightlessness on small mammals over several generations, including hematological effects.

3. On ail missions ail Shuttle personnel should be available as possible subjects for routine medical and performance monitoring inflight. Facilities must therefore be available on the orbiter to conduct metabolic balance studies and to measure, sample, and preserve medical specimens, including urine and feces. This will place requirements on the feeding and waste management systems of the orbiter cabin and on space in the orbiter cabin.

4. The composition of the atmosphere of the orbiter cabin and pressurized laboratory must be monitored continuously for oxygen and hydrogen and periodically for trace contaminants (e.g., $\mathrm{CO}$, hydrocarbons, particularly ethylene, Freons, $\mathrm{O}_{3}$, sulfides, mercaptans, and amines) and viable microorganisms. Trace contaminants and particulate matter, including microorganisms, must be removed to levels consistent with standards recommended in reports of the NRC Committee on Toxicology* and the Space Science Board.t

5. It will be necessary to conduct onboard control experiments for the gravity component and to generate gravity-level response curves for many biological studies. This will require a variable-speed centrifuge with a radius not less than $5 \mathrm{ft}$, capable of producing accelerations up to $1.5 \mathrm{~g}$ and handling plants, tissue cultures, and animals up to $0.5 \mathrm{~kg}$. Since the centrifuge will not be required for every life-science experiment, it should be detachable. from the laboratory.

6. In order to capitalize on the laboratory's potential for animal experimentation, easy access to the animals, for direct manipulation such as for surgery, must be provided. Although an entirely isolated (sterile) environment is unnecessary and impractical, the gas outflow from the animal-holding area should not be discharged directly into the atmosphere inspired by the crew. The animals can be maintained

* Panel on Air Quality in Manned Spacecraft, NRC Committee on Toxicology, Atmospheric Contaminants in Spacecraft (available from NASA Director of Life Sciences, Washington, D.C.).

$\dagger \mathrm{SSB}$ Panel on Air Standards for Manned Space Flight, Atmospheric Contaminants in Spacecraft (NAS-NRC, Washington, D.C., 1968); Physiology in the Space Environment, Vol, II, Respiration (NAS-NRC, Washington, D.C., 1967); Infectious Disease in Manned Spaceflight: Probabilities and Countermeasures (NAS, Washington, D.C., 1970). 
in a closed compartment, with intrinsic environmental control, that could be opened as required.

7. To permit certain biological studies that are time-dependent, late access to the payload is essential. We have been informed that access up to $2 \mathrm{~h}$ prelaunch is feasible, and at the present time this would seem to be sufficient.

8. Dosimetric devices and materials should be positioned in the orbiter cabin on all flights to accumulate data on radiation fluxes and energies and on the effect of diverse spacecraft components on intensities and scattering. While some predictions can be made about the influence of spacecraft materials on the actual radiation exposure parameters, it is necessary to test these expectations against the observed radiation environment.

9. In order to test new or redesigned components of life-support systems at $0 \mathrm{~g}$ in the course of their development, there should be access to the gas-flow loops in the pressurized laboratory.

10. Recent developments in remote manipulation using forcereflecting master-slave servomechanisms and stand-alone force- and tactile-sensitive manipulators have great potential for Shuttle operations and should be applied and encouraged. If this instrumentation is properly developed, remote manipulation can be at least partly substituted for extravehicular activity, with concomitant increases in payload efficiency.

11. In order to permit maximal participation by the research community, criteria for selection of scientific participants should be no more stringent than is necessary to protect the health and safety of the passengers themselves and the crew.

12. There is need for a standardized procedure to inform and to attract potential scientific users of the Shuttle. In addition to the Announcement of Flight Opportunities, there should be announcements in major relevant scientific publications. All proposals for flight experiments and SR\&T should be subject to peer review by panels drawn from the international scientific community. Similarly, peer review of ongoing flight and SR\&T projects should be made at regular intervals.

13. All flight experiments should be preceded by adequate and thorough ground-based preparation. The responses of the test materials must be completely familiar under $1 \mathrm{~g}$ and the other experimental conditions, for in no other way can the quality of the experiment and the legitimacy of any flight results be assured. Thus, 
after an experiment proposal has been accepted, ground-based research and control experiments specific to the flight experiment wili normaiiy be required and shouid be supported.

14. If ESRO proceeds with the Space Laboratory, we strongly urge that life sciences be formally represented in the ESRO management structure. 


\section{9 \\ Planetary Exploration}

\section{O B J E C T I V E S}

The late 1960's and early 1970's were marked by the unique achievements of manned exploration of the moon. In its later stages, the Apollo program provided scientific data that add a new dimension to our knowledge of the earth-moon system.

During the same period, unmanned lunar and planetary exploration, by both the Soviet Union and the United States, provided technological successes comparable to Apollo and scientific returns of similar importance because of the many different objects in the solar system accessible to deep-space probes.

Planetary exploration has, however, only begun, and as long as there is a space program, planetary exploration should continue to be a major objective. Given television, remote operation, and sample return, unmanned operations have a significant capability to extend man's knowledge and experience throughout the solar system.

From the scientific point of view, the aim of planetary research has extended beyond the stimulating but diffuse ambition to understand the origin of the solar system. This remains a major concern, as does the search for life, but of equal importance is the understanding of planetary processes, including those of earth, in a more generalized framework. There is a growing influence throughout the earth sciences of knowledge gained from planetary exploration. New ideas have been introduced, and, more importantly, existing rationales have been challenged. The rapidity with which our knowledge of the earth advances may now be significantly influenced by the rate at which our knowledge of the other planets increases.

The technical achievements of planetary exploration are well illustrated by the spectacular quality of the soundings and images from Mariner 9, by the Soviet landings on the inhospitable surface of Venus, by the Pioneer probes now approaching Jupiter, and by the 
Lunakhod rovers and sample return from the moon. Viking ' 75 to Mars has yet to achieve its objectives; it is probably the most complex instrument package yet designed by man.

A variety of space systems have been developed during this period of technical advance: in the United States, Mariner was designed for high-quality remote sensing, Pioneer for direct measurements and low-cost exploration, the Viking soft lander for surface studies. The Soviet Union developed entry probes and a sample return capability. Pioneer Venus and subsequent missions to the outer planets should establish probe systems also for the U.S. program. Sample return is the next technical challenge for American scientists.

The above space systems must be preserved for use with the Shuttle if we are to avoid continuous redevelopment. In addition, capabilities for sample return and remote operation should be developed in parallel with the Shuttle in order to ensure that an effective program of planetary exploration will be available for use with this new capability.

Some additions to the Shuttle capability will be necessary to ensure adequate escape energies for deep-space missions. These will be discussed in a subsequent section.

In the quest for new information obtainable only from planetary and lunar spacecraft missions, one should not forget the important role played by observations and measurements from earth and from instruments in earth orbit, either on the Shuttle itself or on free-flyers. The essential feature of these observations is that they can be made almost continuously over very long time periods, and thus they can detect subtle changes that could easily escape spacecraft instruments. Thus, synoptic imagery, spectrophotometry, photometry, and polarimetry at high resolution in the optical and ultraviolet ranges are of enormous importance for the study of planetary atmospheric dynamics and chemistry. Infrared observations are particularly important for studies of molecular compositions and reactions. Since these problems and the instrumental requirements are treated in the infrared, optical, and ultraviolet chapters of this report, they will not be discussed here in detail.

Another feature of terrestrial observations that may have significant scientific and philosophical consequences is the search for other planetary systems. Such searches involve the detection of small systematic changes in stellar velocities and proper motions. It is an arduous task that might lead to significant discoveries.

We see four major thrusts in planetary science: comparative planetary science of the inner planets, the nature of a major planet, 
exobiology, and the nature and origin of small objects. Some activity should continue in each of these major areas in order to support an active science community and a stimulating flow of ideas. The specific objectives in each area should be developed by continuing review processes that will identify the next promising target to advance the general field. This assessment was judged inappropriate to the present study, but we believe that NASA and its centers should provide the mechanisms for such review.

The nature of the Space Shuttle creates a greater difference between deep-space exploration and orbital science than has existed up to now, and an effort is required to ensure that NASA's traditional and highly successful commitment to planetary exploration continues.

\section{THE INNER PLANETS}

The last two or three years have brought a new sense of perspective to our knowledge of the inner planets. There is or soon will be sufficient information about the state of differentiation and chemical composition of Mars, earth, and Venus to give hopes for a unified theory of planetary formation. High-resolution images of Mars show features similar to, but essentially different from, those on the earth and the moon-features that tectonic theories must explain. The state of water and carbon dioxide on the inner planets suggests how the atmospheres were formed. The comparative meteorology of Mars, earth, and Venus has become a major concern involving fundamental climatology, tidal theory, nonrotating circulations, clouds, violent storms, boundary-layer phenomena, and other topics. Study of the photochemistry of Mars and Venus has led to a better understanding of the role of interactions between lower and upper atmospheres.

For the inner planets, substantial advances in fundamental knowledge are likely to be achieved from data from all three planets. Despite their similarities, they differ significantly, and measurements on each planet are necessary.

Little is known about Mercury. The first probe to fly by that planet will do so next year. If the history of tectonic processes does not appear to be substantially different from the moon and Mars, the difficulties of making measurements so close to the sun may defer future efforts on this planet in favor of other targets.

For the United States, the major lacuna in past programs has been the absence of a program to explain the surface and lower 
atmosphere of Venus. Entry probes and synthetic-aperture radar mapping using Pioneer and Mariner spacecraft can change this situation. The key to advance, and to the development of probe systems for planetary exploration in general, is the Pioneer Venus multiple-probe mission, followed by orbiters and simple landers. To preserve this program, funding in the fiscal year 1975 budget is essential.

For Mars, the current task is to assimilate the large amount of information available from Mariner 9 with that which will becone available from Viking. The most important complement to the detailed images that are or will be available will probably be surface-sample analysis, preferably at many locations. The tools will be Pioneer hard landers for widespread sample analyses, Viking landers with rover capability, and sample return. For meteorological research, the Mariner 9 instrumentation was well designed, but the lifetime was short and nightside data are scarce. For aeronomy, a Pioneer probe is the ideal entry science mission.

The Viking 1975-1976 soft lander for Mars is the mission chosen for the initial investigation of life in the solar system. Titan, a Saturn satellite, has been identified as another possible habitat for primitive life.

Viking/Mars is an expensive and complex mission. It uses an elaborate approach, which, if it acquires positive signals, could give rise to the most spectacular discovery of the space program to date. The prognosis is not optimisitc, however.

There is no responsible alternative, therefore, but to wait for the results of Viking before planning further biological exploration. If Viking gives tentative signals, there will be strong reasons to continue with the Viking approach, modified in the light of the results achieved. If the signals are negative or confused, it may be preferable to change the approach and emphasize the slow accretion of relevant knowledge, culminating later in an elaborate mission to confirm ideas formed from indirect knowledge. It might also prove desirable to wait for the opportunity to analyze a returned sample. Sample return capability must eventually be available to the U.S. planetary exploration program, and Martian exobiology may be a good first target. Finaily, we may wish to turn attention to Titan when we are more familiar with the exploration of the outer planets.

\section{I. THE MOON}

The Apollo data tell us that the moon formed about 4.5 billion years ago, a time accepted as the age of the solar system. A large 
proportion of the moon consists of compounds that condensed at high temperatures in the solar nebula. For the first few hundred million years of its existence, the moon consisted of an aluminumrich highland crust and iron-rich mantle, both depleted in volatile compounds such as water and methane. During this early period, the moon was heavily cratered by asteroid and meteoroid impacts, of which the largest formed the great circular mare basins. About 0.5 billion to 1.5 billion years later the frontside mare basins, located in a thinner crust than farside basins, filled with basaltic lava, which originated from melting in the deep iron-rich interior. Since that period, the moon has been volcanically quiet; today its seismic energy release is about $10^{9}$ times less than that of the earth. The lunar quiescence has been to our benefit because it has provided an accessible surface on which is recorded the history of meteoroid, cometary, solar-wind, and cosmic-ray activity for over 3 billion years.

This lunar story is fascinating in its own right, but more so because of its applicability to understanding how the earth and other planets formed and evolved. For example, if a planet as small as the moon formed a crust soon after formation, so probably did all planets of similar or larger size such as Mars and earth. In fact, some 2.5-billionto 3.75-billion-year-old aluminum-rich rocks found on earth are now suspected to be remnants of an early crust, one that possibly formed in the same manner as the lunar highlands. It further appears that other old terrestrial volcanic rocks may be equivalent to lunar mare basalts, perhaps representing volcanism triggered by the giant impacts that are suspected to have occurred on the earth 4 billion years ago.

The analysis of lunar soil samples leads us to believe that solar activity has been relatively constant over the past million years; this severely constrains the hy pothesis that the earth's glacial cycles, or ice ages, are related to changes in the sun's activity. Embedded in the lunar soil samples were found solar-wind atoms over 0.5 billion years old, atoms that appear to correspond to today's solar wind. Such atoms are also found in some 4-billion-year-old rocks from the lunar highlands. We thus expect our analyses over the coming few years to provide us with a reliable history of much of the sun's activity since it formed, a record of particular value to our understanding of the evolution of our sun.

The observations of the Apollo astronauts, the lunar samples, and the information continuing to be provided by the instruments left on the lunar surface (such as seismeters, mass spectrometers, and solar-wind detectors), as well as the Explorer orbiters, have answered many of our questions about the moon. But as we learn more about 
the moon, we find new questions to be asked-questions that form the basis for a second generation of lunar exploration.*

Answers to these and other questions about the moon will help us to understand the evolution and nature of our earth, an understanding that contains both scientific and practical significance. Continued analysis and correlation of data from previous studies with new data as it comes in should put us in a position to resume fruitful exploration of the moon by the early to mid-1980's.

\section{THE OUTER PLANETS}

The exploration of the outer planets-Jupiter, Saturn, Uranus, and Neptune-is of great interest for several reasons; the most fundamental is the importance of their overall composition and past history for the reconstruction of the radial, chemical, and thermal structures of the primeval solar nebula. This, in turn, is closely related to the mechanism and chronology for the accretion of the planets. The outer planets contain over 99 percent of the mass and angular momentum of the total planetary system, and thus the structure and composition of their interiors is of great importance. Fortunately, in contrast to the terrestrial planets, the outer planets are large and have low densities so that the possible choices for their chemical composition are very limited. In fact, helium and hydrogen must be their major constituents. As a result, self-consistent theoretical models of the interiors of these planets may eventually be obtained. Uranus and Neptune were once thought to be rather dense, so that their composition was difficult to establish. Recent more accurate optical and occultation measurements indicate that the radii are larger and the densities appreciably lower. In fact, the density of Uranus is lower than that of Jupiter, and that of Neptune somewhat higher. Thus there is hope for obtaining meaningful theoretical models of their internal structure. Pluto is excluded from these considerations because it is small, dense, and in a strongly inclined orbit, which suggests that it is a captured body not characteristic of that part of the solar system.

Among the outer planets, Jupiter is of particular interest because its huge escape velocity and relatively low surface temperature make it a uniquely well-preserved sample of the early solar composition. However, its atmospheric composition, which is susceptible to * See Post Apollo Lunar Science, Report of a Study by the Lunar Science Institute, July 1972, "Statement of Major Unsolved Problems," pp. 27-30. 
remote observation or to spacecraft sampling, does not provide a unique answer about the interior composition, even with respect to the basic problem of the hydrogen-helium ratio.

The second major interest in the outer planets stems from the apparent broad similarity of the structure and dynamics of their atmospheres. Here Jupiter and Saturn stand out not only because they are the biggest planets but also because of the striking analogies and differences between them. The problem of the vertical structure of the deep adiabatic supercritical atmospheres of these planets and of their complicated dynamics has been the subject of innumerable investigations. The wavelength dependence of the limb darkening or brightening appears to be understood. Recently, some insight has been obtained into the mechanism of their atmospheric circulation including the number, stmucture, and appearance of the cloud bands, and many of these phenomena appear to be related to the exceedingly high rotational velocity of these planets. Recently improved observations have led to improved models of the atmosphere of Uranus; but observations and theoretical understanding of Neptune are still in a rudimentary stage.

Interest in the outer planets also focuses on several unique features of Jupiter and Saturn. Among these are the enormous magnetic field of Jupiter and its direct relation to the nature and dynamics of the deep interior of the planet. This magnetic field is closely related to decimetric radiation, which originates in the external radiation belt, and presumably also to the rotating systems of decametric radiation. The mysterious Great Red Spot, known for over 300 years, is another fascinating Jovian phenomenon for which many models have been proposed, although none is completely satisfactory. There is, as yet, no evidence for the presence of a strong magnetic field on Saturn, which could indicate that either the deep interior of that planet is appreciably different from that of Jupiter or that a radiation belt, which would be the best evidence for a magnetic field, is suppressed by the Saturnian rings. The rings themselves, their origin, nature, and dynamics present a fascinating topic for planetary research. The discovery of a significant magnetic field on Saturn would have a profound influence on the admissible models of its interior. The list of the unusual phenomena on Jupiter and Saturn would not be complete without mentioning the enormous source of internal energy that is much higher than the energy received from the sun. There are many proposals that attempt to explain the origin of this energy, from a gradual loss of primordial heat to a continuous 
phase change and gravitational segregation. The source of internal energy is closely related to the still unsolved problem of the radial temperature profile and central temperature of Jupiter. The presence of the internal energy source is well established for Jupiter but not for Saturn, which presents an interesting dilemma in view of the usually assumed similarity of the two planets.

The great variety of questions that need to be answered before the outer planets are reasonably well understood is reflected in the variety of quantitative and qualitative data that are required. For atmospheric studies, one requires information about, among other things, chemical composition, thermal gradients, optical properties, dynamic parameters, and heat fluxes. These should be measured not only as a function of depth but also latitude and perhaps even longitude; probes and radio occultation experiments may be most useful for these purposes. In order to refine our knowledge of the interiors, one needs better gravitational multipole coefficients; oblateness measurements; heat-flux data; information about the magnetic field (electronic and protonic belts); and any indication of local gravitational, thermal, or magnetic anomalies (such as the one recently reported at $220^{\circ}$ longitude). In particular, any information about a north-south asymmetry, either gravitational or thermal, would be significant because of its bearing on the question of the absence or presence of a solid mantle.

None of the required data are obtainable with sufficient accuracy from terrestrial observations and must be deduced from planetary missions. Among these missions, flybys provide the first, rather limited, information; then come the probes, which it is hoped will lead to valuable atmospheric data; and, finally, the orbiters, which alone can give sufficiently complete data to construct satisfactory models of the interior and exterior of each planet. We suggest that the primary focus of the exploration of the outer planets in the period 1977-1990 be placed on Jupiter and Saturn. Our considerably advanced knowledge of these planets permits us to pose sophisticated questions whose answers can have far-reaching consequences. Some preliminary investigation of the more distant outer planets should also be pursued, but it is clear that technical difficulties and cost will make progress slow. During the 1980's, the propulsion and the design of lightweight instrumentation may advance so that in the 1990's the other outer planets will become more accessible.

In view of these remarks, the proposed sequence of missions to outer planets (see Table 19) mission 5 and missions 15 to 21 seems 
reasonable. The flyby missions 5,15 , and 21 will give initial information about Jupiter, Saturn, Uranus, and Neptune, to be followed by probes on missions 16, 17, and 19 to Saturn, Uranus, and Jupiter, and finally orbiter missions 18 and 20 to circle Jupiter and Saturn. It is important that each planet be explored by one orbiter close to the equatorial plane and the other at high inclination. The Saturn probe precedes the Jupiter probe because Jupiter has more than twice the gravitational acceleration of Saturn, leading to many times higher heating of the probe on entry. The Saturn probe is in a sense a test for the new problem of entering the atmospheres of the outer planets.

\section{PLANETAR Y SPACECRAFT}

The exploration of the planets was initiated by NASA in 1962 with the launch of Mariner 2 to. Venus. Since 1962, a plan for planetary exploration has been formulated with the assistance of many science advisory groups. The sequence of missions begins with an exploratory survey by flyby missions, which yield the necessary data to plan more intensive observations by orbiter and entry-probe missions. The next step would be to conduct long-term observations on the surface of a planet such as will be done by the Viking ' 75 mission to Mars. Finally, sample return missions would be initiated for detailed geological and biological analysis of material.

The missions described increase in complexity, and the more complex missions demand greater propulsive capability as well as increased spacecraft capability. A mission to swing by Venus and fly by the planet Mercury was launched in November 1973. Pioneer 11 is currently en route to Jupiter, and Pioneer 10 flew by the planet in December 1973. The Viking '75 mission will place two landers on the surface of Mars to initiate the search for life outside of earth. The Mariner/Jupiter-Saturn program will initiate NASA's high-resolution observations of the outer solar system. The flyby will also permit observations of the Jovian Galilean satellites and the Saturn satellite Titan, one of only three satellites in the solar system known to have an atmosphere.

Currently, three spacecraft systems exist: Pioneer, designed to explore the environment of a planet and to accomplish this with maximum economy; Mariner, either as a flyby or orbiter, designed to obtain high-resolution imagery of the planets and their satellites; and Viking, designed to soft land on Mars and to conduct surface analysis of the planet over an extended period of time. The next step in the 
exploration of the solar system requires development of an atmospheric entry probe. The first such mission planned is a Pioneer spacecraft to deliver probes to Venus. This mission would be followed by measurements in the atmospheres of Saturn, Uranus, and in the satellite Titan. These missions require very modest launch energy capability and can easily be accomplished with a launch vehicle having the equivalent performance of a Shuttle/Centaur system.

The next step in the exploration of Mars might be a roving vehicle on the surface of the planet or a sample return mission. It has been estimated that such missions might weigh some $2000 \mathrm{~kg}$; since the energy requirements for Mars missions are fairly low, they also could be accomplished by a launch vehicle having the capability of a Shuttle/Centaur.

The great success of the U.S. man-driven rover and of the semiautomatic earth-controlled Russian Lunakhod makes it clear that an automatic exploration of the Martian surface should be considered. The essential difference between a mission to Mars and to the moon is the much greater distance to Mars, which makes communication and earth control very slow. The round-trip time in the most favorable case is $18 \mathrm{~min}$, but it could be as long as $40 \mathrm{~min}$. Thus, it is essential to develop an automatic obstacle-avoidance system that would permit the rover to travel to a predetermined destination following a path chosen in detail by the rover's sensors. Such sensors should be able to direct the rover around such things as boulders and steep slopes. An earth-controlled override contingency should also be available. The rover should be able to move on hard and on soft surfaces and should provide protection of the instruments from diurnal thermal variations.

The scientific tasks of the rover could be manifold: TV imagery, thermal and spectroscopic observations, solid and gaseous sample collection and analysis, drilling for deep samples, microscope and x-ray examination, seismological experiments, radioactivity, magnetic observations, and others.

Biological studies may require a sophisticated array of instruments, which, if they are too heavy, too big, or too delicate to be located on the rover, could be left near or in the lander. The possibility of establishing a fairly permanent fully automated station on Mars should also be considered once the technology of remote experimentation is developed. This may not be realizable until the 1990 's. In any case, an automatic adaptive control of the operation of the rover and of the fixed station should be developed. 
Sample return offers many types of information that cannot reasonably be obtained in any other way. The most sophisticated array of instruments that can be landed on the surface of another planet is a poor comparison to the analytical powers of numerous established terrestrial laboratories. The types of sample information that can be obtained from Martian samples can be broadly categorized in three major discipline areas: exobiology, organic geochemistry, and geosciences. The importance of the investigation of the surface of Mars, and returned Mars samples, for the possible presence of extraterrestrial life forms has been elaborated in previous NAS documents (e.g., Biology and the Exploration of Mars*) and is of paramount scientific interest; the probability of a positive result is a speculative matter. Similarly, the analysis of the Martian surface and Martian samples for organic compounds is of extreme interest; the possibility that Martian samples will contain organic compounds of biologic or prebiologic interest is difficult to evaluate. The geochemical, mineralogical, petrological, and geophysical value of the Martian samples is assured. Regardless of their composition, mineralogy, and structure, the samples will be exceedingly informative about the history, surface and internal processes, age, and degree of differentiation of the planet. Analogy with the lunar exploration program demonstrates the tremendous improvement in our understanding of the moon with the return of the first samples from the lunar surface (e.g., see "Proceedings of the Lunar Science Conferences," Geochim. Cosmochim. Acta, Suppls. 1-4).

Recently completed studies by the NASA Langley Research Center and the Jet Propulsion Laboratory indicate that 10 to $1000 \mathrm{~g}$ of Martian sample can be returned to the earth by a Viking-class mission. Although we would like to return the largest possible sample, it should be noted that many of the major conclusions about the moon based on the samples returned by Apollo 11 also derived from the very much smaller Luna 16 and Luna 20 samples. During the past 10 years, NASA has supported a number of lunar sample investigators to improve their abilities to work with very small amounts of returned samples, and many sample investigators now have the ability to derive significant information from individual grains ( 1 to $100 \mu \mathrm{m}$ in diameter). Thus, the information that can be derived from a returned sample of the size contemplated is

*Space Science Board, Biology and the Exploration of Mars, C. S. Pittendrigh, W. Vishniac, and J. P. T. Pearman, eds., NAS-NRC Publ. 1296 (National Academy of Sciences-National Research Council, Washington, D.C., 1966). 
significant. The question of quarantining a Martian sample has been raised. In view of past experience and the new knowledge about Mars that has accuritulated in recent years, it is our judgment that a decision on this question be deferred until the problem can be considered in detail.

\section{PROPULSION REQUIREMENTS}

Each planetary mission has propulsion requirements that depend not only on the distance to the planet and weight of the spacecraft but also on the inclination of the orbit of the Shuttle, the launch period, the path and velocity of the spacecraft in proximity of the planet, and the lifetime of power sources such as the radioisotope thermoelectric generators (RTG's). If the Shuttle provides the first propulsion stage, then the choice concerns only the escape, or upper, stage. The presently considered escape stages include the Centaur and the not yet developed low- and high-performance Tug. Whether the Tug is recoverable or expendable is a distinction that affects the feasibility and cost of the mission. Thrust can be augmented by a kick stage, which is either the existing Burner II or an as yet not developed solid-fuel device. The latter has the advantage of a rapid burn, but it may present structural strength problems during launch. It is expected that at the time the Shuttle becomes operative a $20-\mathrm{kW}$ solar-electric-propulsion (SEP) system will become available.

The approximate weight, size, and cost of the various upper stages are as follows:

Centaur, 3-m diameter, $10 \mathrm{~m}$ long, $14,000 \mathrm{~kg}, \$ 8$ million.

Tug, 5-m diameter, $12 \mathrm{~m}$ long, 27,000 kg (2700 kg empty), $\$ 8$ million low technology, $\$ 17$ million high technology; if reusable, $\$ 1$ million per mission.

Kick Stage (Burner II), $1 \mathrm{-m}$ diameter, $2 \mathrm{~m}$ long, $1400 \mathrm{~kg}, \$ 0.2$ million.

$20-k W S E P, 4.5-\mathrm{m}$ diameter, $6 \mathrm{~m}$ long, $2000 \mathrm{~kg}$, cost as yet unknown.

There are several technical questions to be answered before the upper stages listed above could be accommodated in the cargo bay of the Shuttle orbiter. Outstanding is the compatibility of the weight and size of the spacecraft, escape stage, and kick or SEF stages, par- 
ticularly the location of the center of gravity of the total load. Of special significance are potential problems associated with the inability to vent the Centaur or the Tug in the cargo bay and the problems of thermal control of the RTG's during the flight and the protection of the crew from radiation.

Table 19 lists the planetary missions, their date of launch, their weight and propulsion requirements $\left(M_{0}\right.$ and $\left.C_{3}\right)$ in the ballistic mode and, in certain cases, when using the additional SEP stage. The table indicates also the weight of the delivered payload as well as of the scientific equipment, listing separately the weights of the science on the probes, on the lander, and so on. Figure 5 illustrates the performance of the Centaur (with a kick stage for higher $C_{3}$ ) of the high-technology Tug, either expendable or recoverable, whether with or without a kick stage. In addition, the performance of an expendable low-technology Tug with a kick stage is indicated. The huge differences between the performance of a Tug with or without kick should be noted, as should the relatively small difference between the high- and low-technology Tugs with a kick stage.

Figure 5 also illustrates the propulsion requirements of the various missions that are proposed for the period after 1978. Some of them are indicated without and with a SEP stage, and mission 24 is shown in two variants that differ in the flight time, 800 versus 1100 days. It is clear that Centaur with a kick stage permits the launch of all but two missions to the outer planets, if the SEP is used. Without the SEP, two additional missions would not be possible. In this respect, the comparable performance of the reusable high-technology Tug with a kick stage should be noted.

The extremely important Mariner Jupiter orbiter mission 18 is feasible with a Centaur, but barely so, which results in a rather short launch period of 20 days. Actually this mission is scheduled for an unusually favorable planetary configuration in 1981 and would be impossible with a Centaur otherwise. Mission 21, which is a Mariner Uranus-Neptune flyby, requires the use of an expendable hightechnoiogy Tug. Mission 22, which is an orbiter and lander on the Jovian satellite Ganymede, appears out of the reach of the single-escape stages, and it may require two Tugs that would have to dock in an earth orbit before launch. It should be noted, however, that other Galilean satellites of Jupiter are easier to reach than Ganymede. In fact, they may be reachable without a double upper stage. On the whole, the prospects for a successful launch of missions to the outer planets are very encouraging provided the Centaur 
TABLE 19 Planetary Missions

它

\begin{tabular}{|c|c|c|c|c|c|c|c|}
\hline & & \multicolumn{2}{|c|}{ Ballistic } & \multicolumn{2}{|c|}{ With SEP } & \multicolumn{2}{|c|}{ Payload } \\
\hline & & $\begin{array}{l}M_{0} \\
(\mathrm{~kg})\end{array}$ & $\begin{array}{l}C_{3} \\
(\mathrm{~km} / \mathrm{sec})\end{array}$ & $\begin{array}{l}M_{0} \\
(\mathrm{~kg})\end{array}$ & $\begin{array}{l}C_{3} \\
(\mathrm{~km} / \mathrm{sec})\end{array}$ & $\begin{array}{l}\text { Total } \\
(\mathrm{kg})\end{array}$ & $\begin{array}{l}\text { Science } \\
(\mathrm{kg})\end{array}$ \\
\hline 1 & $\begin{array}{c}\text { Mariner Venus- } \\
\text { Mercury } 73\end{array}$ & 500 & 20 & & & & \\
\hline 2 & $\begin{array}{l}\text { Pioneer Jupiter } \\
\text { flyby } 73\end{array}$ & 259 & 84 & & & & \\
\hline 3 & Helios 74 & 313 & 104 & & & & \\
\hline 4 & Viking 75 & 3375 & 22 & & & & \\
\hline 5 & $\begin{array}{l}\text { Mariner Jupiter- } \\
\text { Saturn flyby } 77\end{array}$ & 750 & 110 & & & & 75 \\
\hline 6 & $\begin{array}{l}\text { Viking orbiter/ } \\
\text { Lander Mars } 79\end{array}$ & 3375 & 22 & & . & & \\
\hline 7 & Sample Mars 84 & 9200 & 16 & $\begin{array}{l}6300 \\
\text { (lander } \\
2350 \text { ) }\end{array}$ & 8 & & $87+26$ (lander) \\
\hline 8 & $\begin{array}{l}\text { Sample Phobos } \\
\text { Deimos } 90\end{array}$ & 3970 & 16 & 3400 & 2 & 500 & $42+26$ (lander) \\
\hline 9 & $\begin{array}{l}\text { Pioneer Venus }+ \\
\text { probe } 78\end{array}$ & 700 & 35 & & & 700 & $47+5$ (probe) \\
\hline 10 & $\begin{array}{l}\text { Other probes }+ \\
\text { orbiter } 80\end{array}$ & 700 & 35 & & & 700 & \\
\hline 11 & Venus radar map 83 & 3150 & 28 & 3550 & 26 & 730 & 123 \\
\hline 12 & $\begin{array}{l}\text { Venus buoyant stat. } \\
\quad 85\end{array}$ & 5615 & 15 & 5700 & 28 & 1470 & 145 \\
\hline
\end{tabular}




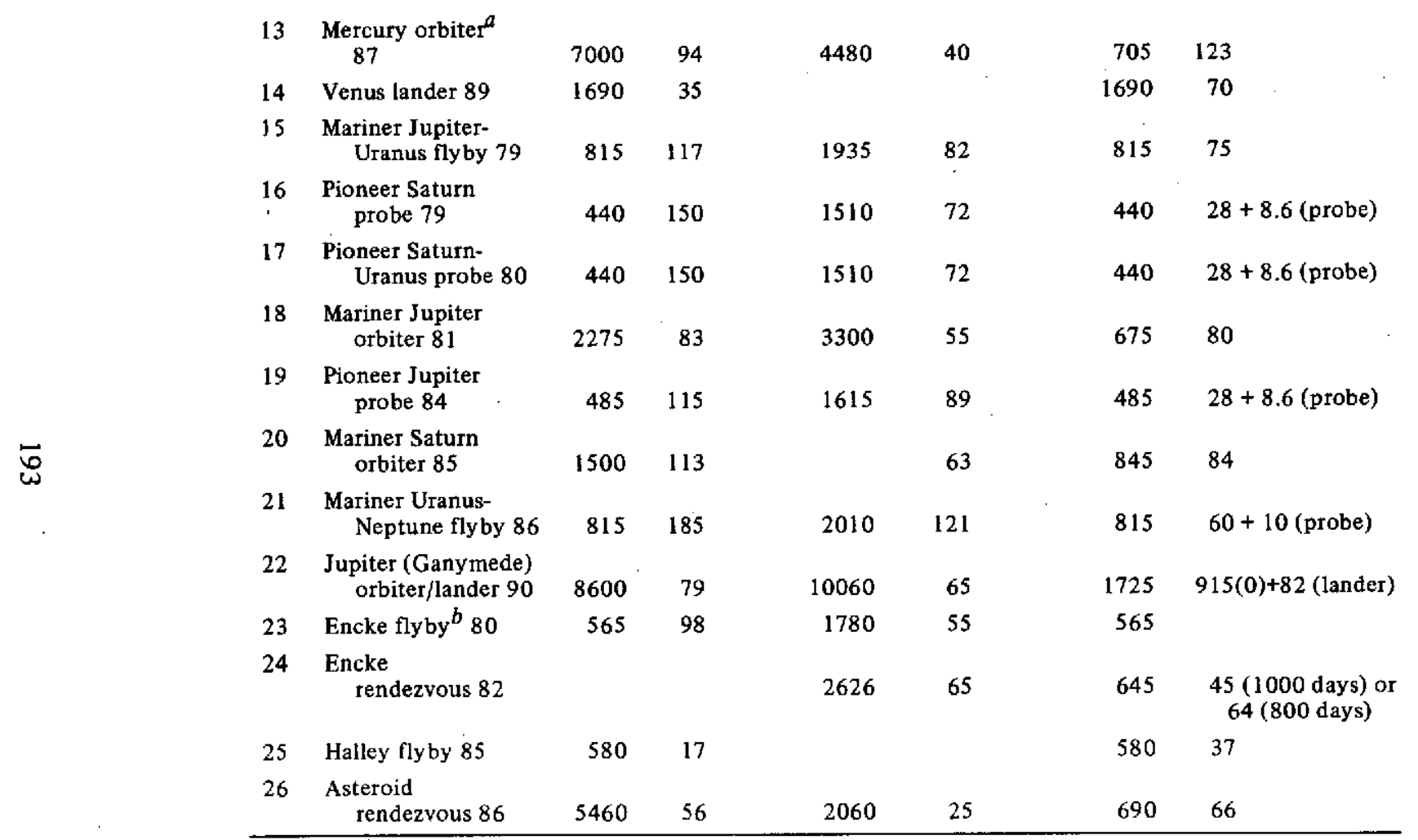

${ }^{a}$ One Tug recovered, one expended.

${ }^{b}$ Velocity at closest approach $7 \mathrm{~km} / \mathrm{sec}$ (ballistic) or $5 \mathrm{~km} / \mathrm{sec}$ (SEP). 


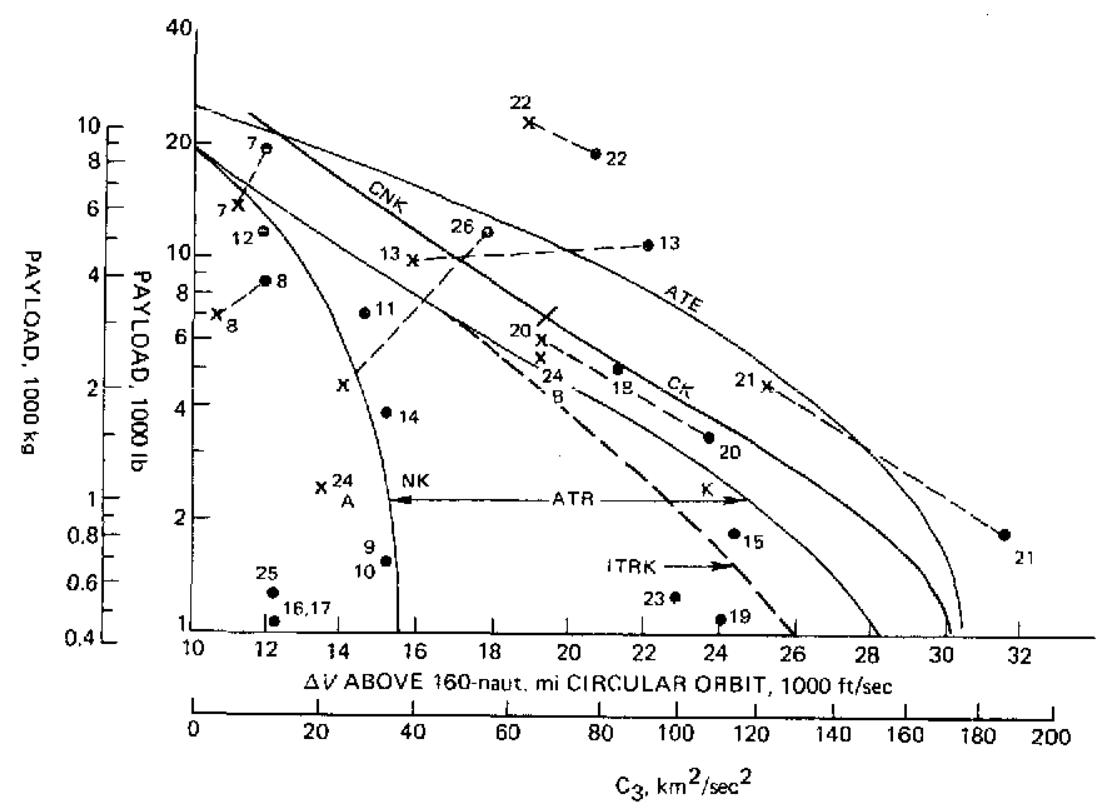

FIGURE 5 Planetary missions propulsion requirements, $\bullet$ Ballistic; X, 20-kW SEP; C, Centaur; AT, advanced Tug; IT, intermediate Tug; $E$, expended; $R$, reused; K, kick stage; NK, no-kick stage. Numbers refer to the missions as listed in Table 19.

capability is maintained, the necessary development and construction of the Tugs and SEP completed, and the problems of transporting them in the cargo bay of the Shuttle orbiter solved.

It should be stressed that the list of planetary missions given in Table 19 is the minimum viable program of planetary exploration in the period 1979-1990. It requires a minimum of 20 to 24 Shuttles fully dedicated to spacecraft launch. The continuation of the excellent lunar explorations will require additional Shuttle launches. It is suggested that in order to assure the observation of suddenly appearing comets, such as the 1973 Kohoutek comet, one payload dedicated to cometary studies should be kept in readiness at all times. The sample return from Mars in 1985-1986 may require the use of a fully equipped laboratory in the sortie mode of the Shuttle. This is a major task that should be investigated at an early date. With additional funding, a series of planetary and lunar missions of comparable or even greater scientific interest could be prepared. 


\section{RE COMMEN DATION S}

1. We recommend the development of the solar electric propulsion (SEP) auxiliary stage for the minimum planetary program; we further recommend a gradual development of Tugs and of other more advanced propulsion methods for a full program of planetary explorations.

2. We recommend that NASA maintain and improve its present spacecraft (Pioneer, Mariner, Viking), develop associated scienceoriented atmospheric probes and automatic rovers, and solve the problem of automatic planetary rendezvous in connection with return of samples from Mars.

3. We recommend that the need for a sortie laboratory for the analysis of a Martian sample be investigated and the laboratory be built if necessary.

4. We recommend that a Shuttle payload be prepared to stand in readiness for cometary studies.

5. We recommend that NASA plan to undertake as a developmental program a block of missions rather than considering two single missions for each opportunity. Such an approach can lead to significant cost reductions. 


\section{Appendix: The Space Shuttle System}

The Space Shuttle is a reuseable transportation system designed to routinely carry scientific and applications payloads to and from low earth orbit in the period after 1980. This system consists of three basic elements: solid rocket boosters, an external tank, and an orbiter. The two solid rocket boosters, after they are expended, are parachute-dropped into the ocean, recovered, and reloaded for subsequent reuse. The external tank, which contains the cryogenic fuels used by the orbiter main engines during launch, is dropped from the orbiter just prior to orbital insertion and is destroyed during its re-entry into the atmosphere.

The orbiter, about the size of a DC-9 airplane (122 ft long), has a pressurized cabin able to accommodate up to ten persons for short missions and a large bay in which to carry payloads. The payload bay has an available volume $15 \mathrm{ft}$ in diameter by $60 \mathrm{ft}$ in length. Within this payload bay the orbiter can carry up to $65,000 \mathrm{lb}$ into a due east orbit and up to $40,000 \mathrm{lb}$ into a polar orbit. In orbit, which is typically $100-200 \mathrm{~nm}$, the payload bay doors open to expose the payload to the space environment. Upon completion of the mission, the orbiter bay doors are closed and the spacecraft is deorbited with the orbiter performing cross-range maneuvers and aerodynamic flight resulting in a horizontal landing at a landing strip. As a transportation system, the Shuttle can

1. Deliver various free-flying spacecraft to orbit and act as a first stage for geosynchronous missions and planetary injection (in addition to deploying spacecraft, it permits functional checkout of spacecraft prior to injection);

2. Revisit previously deployed spacecraft either to service them or to retrieve them and return them to earth for refurbishment;

3 . Carry into low earth orbit a laboratory from which scientific and technical investigations are directly performed. This provides an 
opportunity to carry scientists as passengers to operate their own experiments and also makes possible the repeated use of equipment in the pressurized laboratory or on the pallet.

The Space Shuttle has limited flexibility as to the orbit achieved. From the Eastern Test Range (ETR) $65,000 \mathrm{lb}$ could be delivered to a 210-naut mi circular orbit at 28.5-deg inclination using the basic Shuttle propulsion. A $30,000-1 b$ payload could be placed in a 240-naut mi circular orbit system. By adding one Orbital Maneuvering System (OMS) kit, this 30,000-lb payload could be placed in 350-naut mi circular orbit. Up to three OMS kits can be added to the basic Shuttle system. With three kits, a 30,000-1b payload could be placed in a 510-naut mi circular orbit. From the ETR, orbits with inclinations up to $56 \mathrm{deg}$ can be achieved with some reduction in payload capability over that for a 28.5 -deg orbit.

It is planned that the Space Shuttle will be operated from the Western Test Range as well as the ETR to make possible high-inclination orbits. These high-inclination orbits require greater energy than do low-inclination orbits. The maximum payload that can be delivered into a 90 -deg orbit is $40,000 \mathrm{lb}$, with a maximum altitude without an OMs kit of 145 naut mi circular. By adding a single kit, the $40,000-1 b$ payload can be lifted to 300 naut mi circular.

To ensure safe supersonic and subsonic flight and landing, certain constraints are placed on the weight and location of the returned payload. The maximum returned payload is limited to $32,000 \mathrm{lb}$. A $32,000-1 \mathrm{~b}$ payload would have to have its center of gravity located between about 32 and $44 \mathrm{ft}$ behind the forward payload bay bulkhead. Heavier payloads may be landed in an abort situation, with some degradation in safety, providing the payload center of gravity is contained within the so-called 2 percent envelope. This means that a $65,000-1 \mathrm{~b}$ payload could be landed in abort if its center of gravity is located between 38 and $44 \mathrm{ft}$ behind the payload bay forward bulkhead. 